की

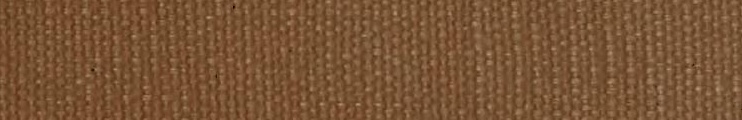

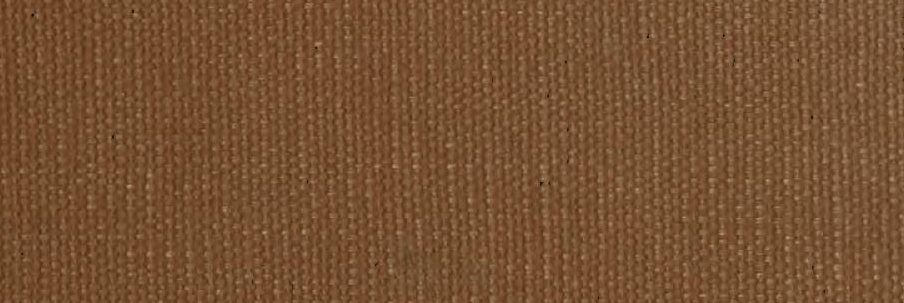

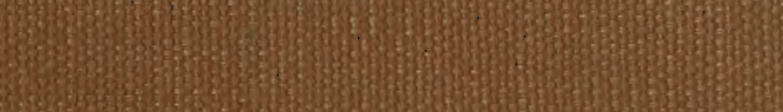

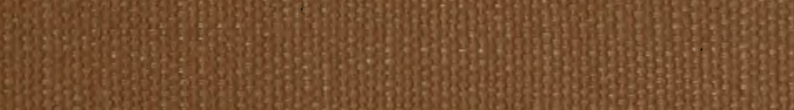
2035)

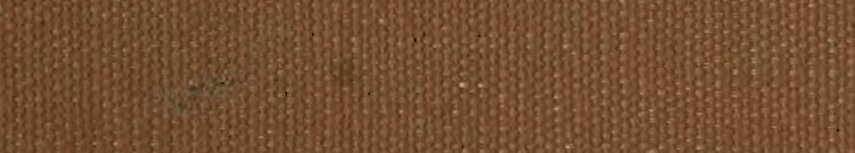

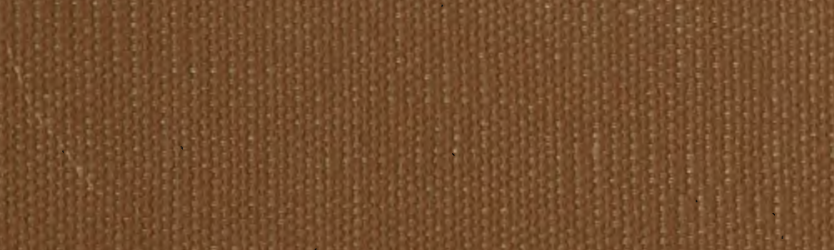

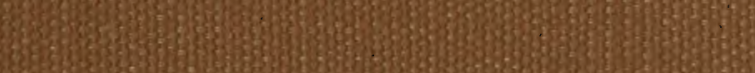
1. *3.5. C8:-2. 3.

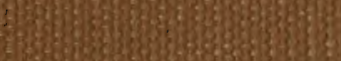







Dufour:

Pecte nuat. es pheys. sur les eipteres. 



\title{
RECHERCHES ANATOMIQUES
}

\author{
ET PHYSIOLOGIQUES \\ SUR LES DIPTERES,
}

ACCOMPAGNÉES

DE CONSIDÉRATIONS RELATIVES A L'HISTOIRE NATURELLE DE CES INSECTES

\author{
PAR M. LÉON DUFOUR, \\ DOCTEUR MÉDECIN,
}

CORfespondant de Linstitut (académie des sCiences).

"In his tam parvis atque tam nullis quæ ratio! quanta

rvis! quam inextricabilis perfectio !n (PLINE.)

\section{INTRODUCTION.}

Dans ce siècle d'argent et d'esprit, qui est loin d'ètre l'âge d'or, qui daignera laisser tomber un regard, même de simple curiosité, sur le cerveau d'une mouche, les organes génitaux d'un cousin, les entrailles d'un ver de la viande? A ces mots, l'homme du monde hausse les épaules et sourit de pitié; mais les hommes de science écoutent, se recueillent et comprennent que dans cette échelle zoologique, où tous les organismes s'enchaînent, s'anastomosent, la mouche, le cousin, le ver, ont un rang assigné, et que celui qui consacre ses veilles à mettre en évidence les affinités et les dissemblances organiques qu'ont ces petits êtres 
entre eux et avec les autres animaux, avec l'homme lui-même, a quelques droits à une attention sérieuse.

C'est une nouvelle histoire des insectes que celle qui embrasse les études simultanées et parallèles des formes extérieures et de l'organisation intérieure. Déduire rationnellement les habitudes et le genre de vie de la structure et de la combinaison des organes renfermés dans les cavités du corps, et préjuger de l'existence de ces organes par les actes de l'animal, c'est là, incontestablement, une science de haute philosophie. Depuis vingt-cing ans, j'envisage l'étude de l'entomologie danś cet esprit, après m’ètre adonné pendant longtemps à la connaissance des genres et des espéces. J'ai successivement publié l'anatomie des Coléoptères, des Labidoures, des Hémiptères, des Orthoptères, des Hyménoptères et Névroptères; je viens présenter aujourd'hui, au jugement de l'Académie des sciences, celle des Diptères. Il ne me restera plus à disséquer que les Lépidoptères pour avoir soumis aux recherches de mon scalpel les huit ordres qui composent l'entomologie proprement dite, c'est-à-dire les insectes hexapodes. Dans cing ou six ans j'aurai, je l'espère, rempli cette tâche.

O sagesse infinie! en jetant avec profusion sur notre planète ce peuple immense des Diptères, qui pour le vulgaire se réduisent aux mouches; en assignant à chaque famille, à chaque groupe, sa nourriture, son genre de vie et son mode de propagation; en les dotant d'une organisation conséquente à ce triple but, tu n'as pas dédaigné de les faire concourir aux sublimes harmonies qui régissent l'univers. Laissons donc tant d'hommes qui ne sont pas appelés à te comprendre s'épuiser en plaintes inutiles, décrier des cuvres quils ne veulent ou ne peuvent pas connaitre; laissons-les avec leur éternel cui bono, dont l'immortel Linné a si bien fait justice, et poursuivons avec gravité l'étude de tes plus minimes productions, parce que c'est précisément là que ton génie nous révèle tes plus sublimes conceptions.

Considérés sous le point de vue du nombre des espèces et des 
individus, les Diptères sont, de toute la zoologie, l'ordre d'animaux le plus répandu sur le globe. Leurs larves pullulent dans toutes les matières animales ou végétales en décomposition, ainsi que dans les corps organisés vivants eux-mêmes, et il n'est pas de conditions de sol et de température qui ne soient peuplées de leurs cohortes ailées. La Providence leur a confié, n'en douttons point, une grande, une importante mission, et lorsque Linné disait qu'un lion ne dévorait pas plus vite un cadavre que ne le feraient trois mouches de l'espèce de celles qui mettent au monde des milliers de vers vivants, son assertion n'était pas aussi hyperbolique qu'on pourrait le croire.

Voyez comme la puissance créatrice a tout calculé, tout prévu, dans un but général de conservation et d'harmonie! comme elle sait rapprocher d'un mal inévitable un reméde nécessaire! Ce vaste marais qui répand au loin ses miasmes délétères a pour correctif la production incessante de l'oxygène par les saules, les roseaux de sa rive, par les typha, les scirpus, les nymphæa de ses eaux; mais par le fait même de l'envahissement de l'élément liquide par ces végétaux, il en résulte une plus grande stagnation de l'eau, une macération de leurs dépouilles, une décomposition organique; un foyer de nouveaux dégagements méphiliques et aussi un berceau de nonveaux êtres organisés : le correctif est encore là. Ces myriades de mouches, à haljitudes sédentaires, s'occupent à rendre à la vie ces atomes décomposés, à les passer à l'alambic de leurs organes digestifs, à les translormer en éléments nutritifs, à diminuer ainsi la somme de matière putréfiable. Admirons donc, si nous ne savons pas le comprendre, ce cercle éternel de circonstances où la vie et la mort, toujours aux prises, amènent en définitive la conservation de l'existence et le maintien des harmonies.

Les exigences scientifiques de l'époque m'ont fait attacher la même importance à l'autopsie d'un moucheron qu'à celle d'un quadrupède : la taille ne fait rien au sujet. Dans l'anatomie de chaque famille des Diptères, je ne me suis pas borné à une sèche 
exposition matérielle des organes; j’ai cherché à rattacher le nombre, la structure et la combinaison de ceux-ci à la classification établie ou à établir; jai fait aussi marcher de front les considérations physiologiques toutes les fois que la connaissance des formes ou des actes extérieurs se prêtait it leur application. Cette conformité des viscères avec le genre de vie est surtout l'objet de mes investigations. Il est beau de rencontrer dans ces mouches um plan d'organisation qui les rattache si admirablement aux animaux considérés comme les plus parfaits que, pour la description de leurs appareils de la vie, on peut leur adapter la nomenclature anatomique consacrée depuis des siècles. Ceux-lá seuls dont le scalpel s'est voué avec une patience imperturbable à cette microtomic qui m'est devenue familière, se feront une juste idée des vives jouissances que procurent et la découverte des faits de concordance dont je viens de parler, et celte marche succesive de la nature dans ses créations.

Dans l'exposition de mes recherches anatomiques j’ai suivi, quant à la série des genres, l'ouvrage de M. Macquart, intitulé: Histoire naturelle des insectes diplères'. C'est le tableau le plus complet de la classification des insectes de cet ordre. Je n'ai pas cru devoir adopter toutes les réductions que cet auteur, trop inspiré peut-être par les derniers ouvrages de Latreille, a fait subir aux familles primitivement établies par celui-ci ou par le célèbre diptérologiste Meigen ${ }^{2}$. Sans commettre la moindre infraction à la série si naturelle des groupes nombreux fondés par M. Macquart, je mo suis permis de restituer à quelques-uns d'entre eux les noms de familles consacrés déjà dans l'immortel Genera de Latreille el adoptés par plusieurs entomologistes.

Je n'ai pas manqué non plus de consulter le travail de M. Rohineau-Desvoidy sur les Myodaires ${ }^{3}$. Ce livre, effrayant au pre mier abord par l'excessive multiplication des genres et les signa-

1 Deux vol, in- $8^{\circ}$, librairic de Roret, 1835.

- Dipt. curop. sept vol, avec pl. $1818-1838$.

* Essai sur les Myodaires, Mémoires de l'Institut, J S3o. 
lements trop restreints des espèces, se recommande par des aperçus d'un piquant intérêt sur les mœurs, les habitudes de ces Diptères et sur le rôle qu'ils jouent dans la nature. Il est fảcheux qu'on ait à lui reprocher l'absence de presque toute synonymie: c'est là, suivant moi, un délit scientifique.

Nes recherches reposent sur des milliers de vivisections, pratiquées sur cent quatre-vingt-quinze espèces choisies dans les principaux groupes de l'ordre, en sorte qu'il a été permis de s'élever nvec quelque certitude à des généralisations. Il importait à ma responsabilité d'auteur, il importait à la science, que ces espèces fiussent rigoureusement dénommées, soit pour alléger mon texte des longueurs de descriptions spécifiques, soit dans l'intérêt du contrôle de mes observations. J'ai recouru pour cela à la source la plus sûre, la plus authentique, et M. Macquart a daigné luimême ou confirmer ou établir la nomenclature de tous les Dipières qui ont passé sous mon scalpel.

Pour abréger mon texte, sans le rendre moins substantiel, j’ai dû traiter dans des chapitres spéciaux les appareils organiques qui ne se modifient pas assez suivant les familles pour se prêtes a des descriptions détaillées, comme les appareils sensitif et respiratoire, et le tissu adipeux splanchnique. Dans ce même but d'éviter d'oiseuses répétitions et de fixer la valeur de quelque: dénominations anatomiques, j'esquisserai à grands traits les organes de la digestion et de la génération. Il résultera de là que l'ensemble de mes recherches se partagera en deux grandes divisions: Anatomie générale et anatomie particulière des familles.

Mon scalpel, en pénétrant dans ce monde nouveau d'organismes, n'a pas la prétention d'avoir reconnu les formes et les structures, même les plus générales. A peine ai-je défriché la superficie du champ. Il y a encore immensément à faire. 


\section{PREMIERE DIVISION.}

INATONIE GÉNÉRALE.

\section{CHAPITRE PREMEN.}

AYPAREIL SENSITIF.

Dans l'exposition du système nerveux des Diptères, je vais prouver combien jusqu'à ce jour on avait des connaissances vagues, des idées fausses sur sa composition et sa structure, et dans combien d'hérésies physiologiques on s'est jeté pour avoir voulu éiablir des règles générales sur des faits trop peu nombreux et mal compris. Ainsi, les uns ont avancé que les Diptères avaient neuf ganglions, les autres un seul, et tous, entrainés par une application hasardée de la loi de l'analogie, ont dit que ces ganglions étaient séparés par un double cordon. Il y a dans ces assertions grande inexactitude et erreur flagrante. Oui, il est des Diplères où l'on trouve neuf ganglions, sans y comprendre le cerveau, et d'autres où il n'y en a qu'un; mais ce ne sont pas là toutes les combinaisons, et je vais en faire connaître où ce nombre est de sept, de six, de cinq, de trois, de deux, d'un seul; enfin, il y a des larves où on en compte onze et même douze. Ces centres nerveux sont, dans tous les Diptères, unis et séparés par un cordon inter-ganglionnaire très-simple et non double. C'est même là le trait distinctif de cette chaine de ganglions avec celle des autres ordres d'insectes.

Venons aux faits; voyons si le nombre des ganglions est en harmonie avec la classification établie, et quelle peut être son importance pour celle-ci.

M. Nacquart a partagé tout l'ordre des Diptères, d'après la considération des antennes, en deux divisions : l'une, les Némocères; l'autre, les Brachocères. Mais, indépendamment de ce que les antennes de plusieurs Brachocères ont, dans le fait, plus de trois 
articles, l'anatomie, et surtout la composition du système nerveux, rendent inadmissible une division aussi absolue, aussi générale.

Dans les deux familles des Culicides et des Tipulaires, le système nerveux a un degré, sinon de développement, du moins de composition, qui semble témoigner de la prééminence organique accordée à ces Diptères. Il se compose du cerveau avec un bulbe rachidien, de neuf ganglions et des diverses paires de nerfs qui partent de ces centres nerveux. Je vais plus particulièrement décrire et figurer cet appareil dans la Tipula oleracea, tout en prévenant que j'en ai constaté l'identité dans plusieurs grandes et petites espèces, en sorte que ce type d'organisation pourra être considéré comme un attribut de ces deux populeuses familles.

Le cerveau ou l'organe des fonctions sensoriales est étroitement enveloppé par la boîte crânienne et formé de deux hémisphères égaux séparés par une profonde scissure médiane, mais réunis, confondus inférieurement par une continuité de substance. Ne sont-ce pas là des traits que le Diptère partage avec les animaux de l'ordre le plus élevé? Déchirez l'enveloppe tégumentaire pour en dégager l'encéphale; les lobes de celui-ci, obéissant à une certaine élasticité ou expansibilité jusque-lì maîtrisée, s'écartent l'un de l'autre et prennent la forme de deux sphéroïdes unis par leur partie inféricure. C'est ainsi que les représente la figure que j'en donne. Ces lobes ou hémisphères h̀offrent extérieurement aucune trace de ces plis sinueux, de ces circonvolutions qui caractérisent ceux des quadrupèdes. Ils sont lisses et blancs, mais leur substance est sensiblement plus pulpeuse que celle des ganglions. Dans leur position normale, un grand segment de sphère de leur surface supérieure et antérieure est caché par les rétines oculaires. Les nerfs optiques, dont celles-ci ne sont que l'épanouissement, ont une excessive brièveté et ne sauraient être mis isolément en évidence. Les hémisphères cérébraux se terminent en arrière par deux prolongements fort courts, dans l'intervalle desquels passe l'osophage: c'est ce qu'on appelle le collier asophagien. La rétine 11. 
oculaire, qui est enchatonnée sous la cornée, a un pigment violacé dont la réticulation est parfaitement conforme aux aréoles de cette dernière. Je parlerai ailleurs de la choroüde et des cristallins.

Mais, indépendamment de cette rétine, notre tipule m'a offert un fait curieux : il existe au bord postérieur de chaque rétine oculaire un petit nerf optique ocellaire terminć par une rétine subglobuleuse à pigment violacé. Ce qui rend ce fait anatomique piquant, c'est que dans la Tipula oleracea, ainsi que dans toutes les espèces du genre Tipula tel qu'il a été circonscrit par Meigen et M. Macquart, il y a absence complète d'yenx lisses, et ce trait négatif est exprimé dans le signalement générique exposé par ces entomologistes. Depuis la découverte de ces nerfs ocellaires, j’ai dirigé les explorations les plus scrupuleuses vers la région de la tète des Tipula, qui, dans d'autres tipulaires, est le siége haljituel des ocelles; je les ai renouvelées et sur les individus des deux sexes dans l'état de vie, et sur ces mêmes individus peu ou longtemps après leur mort, et la loupe la plus forte ne m'a décelé aucun ocelle. Toutefois, j'observe derrière l'insertion de chaque antenne de notre tipule une fort petite saillie subhémisphérique, simplement tégumentaire. Cette protubérance crânienne est-elle le réceptacle, l'opercule de la rétine ocellaire? La position respeclive de ces deux protubérances avec les yeux de la tipule est bien différente de celle où, dans mes dissections, j’ai trouvé et représenté les nerfs ocellaires. Mais, comme l'isolement du cerveau ne peut s'opérer que par un grand dérangement de ses parties, il est possible, il est même vraisemblable que les rapports de l'optique ocellaire avec la rétine oculaire ont été violés. J'ajouterai à l'appui de l'idée qui tendrait à considérer ces éminences tégumentaires comme les opercules des rétines ocellaires, qu'il y a conformité de volume et de configuration entre les unes et les autres. Observez encore une anomalie dans l'existence de ces optiques ocellaires, c'est qu'il n'y en a que deux, tandis que dans les 'Tipulaires pourvues d'ocelles, ceux-ci sont presque toujours au nombre de trois. Ainsi, il faut envisager les optiques ocellaires et les pro- 
tubérances crâniennes dont il est question comme des organes imparfaits dépourvus de fonctions. Ce sont des organes vestigiaires, des jalons anatomiques qui témoignent hautement de la gradation qui préside au plan général des créations.

Revenons au cerveau de notre tipule. Du bord antérieu de chacun des hémisphères partent deux nerfs bien distincts : l'un, antennaire; l'autre, buccal. C'est en arrière et en dessous que ces hémisphères confluent ensemble, et à l'endroit de cette confluence existe un troisième lobe ganglioniforme que j'ai cru pouvoir désigner par le nom de bulbe rachidien, n'osant pas l'appeler cervelet, quoiquil ait une texture identique avec le cerveau. On ne saurait le prendre pour un ganglion, à cause de la continuité directe el large de sa substance avec ce dernier.

La chaine ganglionnaire se compose de ganglions thoraciques et de ganglions abdominaux. Ces ganglions, sauf les cas où il y a contiguïté de quelques-uns d'entre eux, sont séparés les uns des autres par un cordon nerveux très-simple qui n'en est qu'une atténuation. La simplicité de ce cordon est, je le répète, un caractère différentiel de l'ordre des Diptères avec les autres ordres d'insectes. Il n'est pas rare de découvrir, le long de la ligne médiane du cordon, une trachéole simple, fine comme un brin de soie, qui peut en imposer et qui m'en imposa d'abord, pour la trace d'une division en deux filets contigus. Son aspect resplendissant dissipe l'illusion. L'existence de cette trachćole est encore, à mes yeux, un vestige, un léger mais précieux souvenir anatomique, que la nature a laissé sur son passage, des créations échelonnées.

Les ganglions thoraciques sont au nombre de trois, mais soudés, presque confondus en une masse oblongue à trois légers festons latéraux, profondément enchâssée entre les saillies ou apophyses coriacées qui correspondent aux insertions des pattes, de manière qu'il est fort difficile de l'isoler dans son intégrité. Ils occupent le centre du thorax. Chacun d'eux émet une paire principale de nerfs cruraax. 
II y a six ganglions abdominaux arrondis, sublenticulaires, égaux entre eux, à l'exceplion du dernier, qui ici, comme dans tous les insectes en général, a une grandeur presque double des autres. Ces ganglions fournissent chacun une paire de nerfs. Outre celle-ci, le dernier se termine par deux grands nerfs génitaux.

Dans les larves du Xyphura et du Pachyrhina, et sans doute des autres grandes Tipulaires, le nombre des ganglions est supérieur a celui de l'insecte ailé, puisqu'il est de onze, le cerveau non compris. Quelle induction tirer de cette prédominance numérique des centres nerveux dans ce premier âge de l'insecte que l'on s'accorde à considérer, el avec raison, comme un état imparfait? Je ne puis le dire; mais il y a encore beaucoup à étudier. La corrélation que l'on a cru exister entre le nombre des ganglions ef celui des segments du corps ne saurait être prise en sérieuse considération. J'ai prouvé dans mes recherches anatomiques sur les Hémiptères, les Orthoptères, etc. et je prouverai dans le chapitre actuel qu'elle est fort loin d'être une règle, puisque, pour le dire en passant, un fort grand nombre de larves de Diptères qui ont douze segments au corps n'ont qu'un ganglion unique. Quoi quil en soit, le cerveau de nos larves de Tipulaires n'est point renfermé dans la tête et est dépourvu de rétines, puisque ces larves n'ont pas d'yeux. Il consiste en deux sphéroïdes contigus, confluents par leur partie inférieure et séparés de la chaine gangliomnaire par le collier oeșophagien. Après celui-ci, il existe une série de cinq ganglions contigus, arrondis, logés dans cette région de la larve qui correspond au futur thorax, et il est bon de se rappeler que le corselet de l'insecte ailé n'a que trois ganglions. Les abdominaux sont au nombre de six, émettant des paires de nerfs, que la figure indiquera suffisamment.

Malgré son extérieur musciforme, le Bibio, placé aux confins des Diptères némocères avec les Brachocères, se rattache, par son système nerveux, à la famille des Tipulaires, où on l'a colloqué à bon droit; mais il va nous offrir une de ces transitions organiques si intéressantes it mettre en relief. Cet insecte a six 
ganglions abdominaux distincts; mais au lieu des trois ganglions thoraciques soudés, propres aux Tipulaires légitimes, il n’en existe que deux séparés l'un de l'autre, quoique rapprochés. Le p̉lus postérieur est grand et arrondi. Ce même nombre existe aussi dans le Sciara, et quoique je ne l'aie pas constaté dans le Rlyyplus, l'analogie viscérale et le poste occupé par cette Tipulaire florale dans le cadre entomologique me porlent à croire qu'il offrira une semblable disposition. Cette différence numérique des ganglions thoraciques dans les dernières Tipulaires nous conduit, comme par la main, au groupe qui les suit dans la série.

La famille des Tabaniens, qui suit les Tipulaires, a sa chaine ganglionnaire de sept ganglions seulement; par conséquent, elle en a deux de moins que ces dernières: je n'en conclus pas cependant que les Tabaniens, insectes robustes et sanguinaires, aient une organisation inférieure à celle des Tipulaires. Leur système nerveux a un développement, une masse cérébro-rachidienne et une concentration de la pulpe nerveuse qui pourraient bien balancer avec quelque avantage la multiplicité des centres nerveux. C'est là une question physiologique que je n'entreprendrai pas de résoudre en ce momẹt. Je prendrai pour type de ma description le Tab. bovinus, et je ne reviendrai pas sur les divisions et les détails de structure que j’ai exposés dans les Tipulaires.

Son cerveau, à cause du grand développement des yeux, est, dans sa situation naturelle, presque entièrement recouvert par les rétines oculaires, et il faut le renverser, ainsi que le représente l'une de mes figures, pour mettre ses hémisphères en évidence.

Comme j’ai eu occasion d'étudier dans cet insecte les parties constitutives de l'œil, j'en dirai deux mots sans prétendre traiter à fond cette question. La choroide ou l'uvée de Sivammerdam est un tissu membraniforme violacé, intermédiaire à la cornée ef it la rétine. C'est un organe comme parenchymateux, sur lequel Muller nous a donné des notions bien plus positives que ses de- 
vanciers; sa configuration est parfaitement celle de la cornée, dont elle tapisse toute la surface interne. Cet organe se détache si facilement, par la macération, des surfaces avec lesquelles il est en contact, qu'on croirait, au premier coup d'œil, que ses connexions se bornent à une simple contiguité; mais une étude attentive prouve que les divisions fragiles et insaisissables du nerf optique, ainsi que les trachéoles nutritives les plus fines, le pénètrent de toutes parts. Sa surface sous-cornéenne parait alors velue, veloutée, à cause de la saillie des cristallins, qui ne m'ont pas paru des cônes, comme les appelle Muller, mais des cylindres hexagonaux étroitement pressés entre eux et en nombre égal à celui des cellules de la cornée; sa surface rétinéenne est élégamment brodée par des trachées rayonnantes d'où partent, sans doute, les trachéoles nutritives qui se distribuent aux cristallins et au pigment violet. Mes figures me dispensent de m'étendre sur ce point.

Après le collier æesophagien vient te bulbe rachidien, suivi d'un cordon simple assez long, qui fournit trois paires de petits nerfs. Il n'existe qu'un seul ganglion thoracique, mais grand, ovale-elliptique, émettant sept paires de nerfs et représentant les trois ganglions soudés des Tipulaires. Le chapelet abdominal n'est que de cinq ganglions, mais le dernier est évidemment formé par la fusion de deux, ainsi que le prouve le nombre de nerfs qu'il fournit. Ce chapelet présente cette disposition singulière, qu'au lieu d'être tout renfermé dans la cavité abdominale, il se trouve en grande partie dans le thorax et à cheval sur le détroit thoracoabdominal, de manière que le dernier ganglion ne dépasse pas le second segment ventral. Ces ganglions ovales arrondis sont d'autant plus rapprochés entre eux qu'ils sont plus postérieurs; l'avant-dernier et le dernier sont même contigus. Chaque ganglion abdominal fournit par ses angles postérieurs une paire de nerfs dirigée en arrière. Cette direction est la conséquence de la situation des ganglions abdominaux dans le thorax. Elle prouve la légitimité de leur dénomination, en même temps qu'elle dépose contre l'idée que cette position à cheval entre les deux cavités 
pourrait être accidentelle : je l'ai, d'ailleurs, confirmée dans plusieurs espèces de Tabanus. Le dernier ganglion se termine par un cordon médian assez long d'où naissent symétriquement six paires de nerfs.

Dans le Pangonia, qui diffère surtout du Tabanus par la longueur de son suçoir, la chaine abdominale est de six ganglions distincts et séparés, tous renfermés dans la cavité et à égale distance les unś des autres, à l'exception du dernier. Dans la femelle de ce Pangonia, les trois derniers ganglions sont moins distants entre eux que dans le mâle. J'aurai occasion bientôt de signaler des différences plus remarquables du. système nerveux suivant les sexes ${ }^{1}$.

La famille des Stratyomides, dont jai étudié le système nerveux, surtout dans l'Ephippium, a le même nombre, la même disposition des ganglions rachidiens que le Pangonia de la famille précédente, c'est-ḋ-dire un thoracique et six abdominaux distincts. Dans les Odontomyia et le Vappo, je n’ai constaté que cinq de ces derniers, le terminal ovalaire plus grand; dans le Chrysomyia, six, dont les trois derniers contigus.

On retrouve dans la famille des Asiliques (Laphria fulva, Dasypogon punctatus) la même composition numérique de la chaîne gangliomaire que dans les Tipulaires, savoir : neuf ganglions, dont trois thoraciques contigus, mais non soudés, et six abdominaux bien séparés.

Nous avons vu que les larves des Tipules avaient deux ganglions de plus que les insectes ailés. La larve d'un Asilique (Laphria atra), dont je réserve pour un mémoire particulier l'histoire des métamorphoses et de l'anatomie, en a trois de plus. Indépendamment du cerveau, il y a cinq ganglions thoraciques non contigus et sept abdominaux.

Je n'ai trouvé dans le Cyrtus que quatre ganglions abdominaux; les deux derniers plus rapprochés.

1 J'ai reconnu à l'origine supérieure du ventricule chylifque du Tab. bovinus un ganglion lenticulaire qui se rattacbe au système nerveux stomato-gastrique de Brandt; mais je a'ai pas des observations assez précises pour en exposer la description. 
Le système nerveux des Bombyliers a la plus parfaite analogie avec celui des Asiliques: trois ganglions thoraciques et six abdominaux. Celui des Anthraciens, qui les suivent immédiatement, est semblable à celui des Stratyomides: un ganglion thoracique fort grand et six abdominaux.

Que penser de cette famille des Brachystomes, fondée par M. Macquart, avec des types si mal assortis, si antipathiques, lant pour les formes extérieures que pour le genre de vie et l'organisation viscérale? Peut-on ne pas reconnaitre d'invincibles répugnances entre le Thereva, le Dolichopus, le Syrphus, enfermés dans la mème enceinte? L'étude comparative du système nerveux s'oppose formellement à.cette alliance.

Dans les Thérévides et les Leptides, la série des ganglions est conforme à celle des Stratyomides; mais, dans les premiers, les deux derniers abdominaux sont soudés, tandis qu'ils sont séparés dans les Leptides.

La belle famille des Syrphides ne ressemble pas du tout pour la composition de son système nerveux aux deux précédentes. J'ai surtout étudié ce système dans le Volucella, et je l'ai confirmé dans les Eristalis, Syrpluus, Rhingia, Cheilosia, etc. Il consiste en trois ganglions rachidiens, un thoracique et deux abdominaux. Le thoracique occupe le tiers antérieur du thorax : il est grand, ovalaire, et émet six paires principales de nerfs et plusieurs petites. Les abdominaux ont leur premier petit, placé sur le troisième segment ventral et ne fournissant qu'une seule paire de nerfs; il est séparé du thoracique par un fort long cordon qui ne m'a paru donner naissance à aucun nerf. Le dernier, presque aussi grand que le thoracique, est situé au tiers postérieur de l'abdomen : il en nait quatre paires de nerfs.

Le genre Scenopinus semble avoir été mis au monde pour le tourment et le désespoir des classificateurs: c'est une pomme de discorde lancée dans l'arène entomologique. Il est certainement plus facile de dire là oú cet insecte se trouve déplacé que de lui assigner son véritable rang dans le cadre. Il faut encore le con- 
sidérer comme un Diptère à parti prendre, comme une famille errante et nomade. Qu'il ine suffise en ce moment d'annoncer que son système nerveux ne ressemble ni à celui des Syrphides, qui le précèdent, ni à celui des Conopsaires et des Muscides, qui le suivent: il aurait plutôt des rapports avec les Thereva. Il a cing ganglions abdominaux distincts (au lieu de six); le dernier, plus grand, a peine un peu plus rapproché de colui qui le préréde.

La famille des Conopsaires, à laquelle, à l'exemple de Latreille, je réunis les Myopa, a un appareil sensitif qui justifie pleinement cette union. Indépendamment du nombre fort restreint de ses ganglions rachidiens, cet appareil va nous offrir un fait bien singulier : c'est que sa disposition et sa distribution sont fort différentes suivant les sexes. Je décrirai celui du Conops rufipes; mais j’ai constaté sa conformité dans le Myopa ferruginea.

Les conopsaires n'ont que deux ganglions. Le thoracique est, dans les deux sexes, grand, ovalaire, enchatonné au milieu du thorax, et fournit trois paires principales de nerfs. Dans le mâle, le cordon interganglionnaire, thoraco-alsdominal, est simple d'un bout à l'autre, et bien plus court que dans la femelle; celle-ci a ce même cordon pareillement simple dans son trajet du thorax, où il émet deux paires de nerfs; mais, à son entrée dans l'abdomen, il se divise aussitôt en deux longs filets, qui demeurent distincts et séparés jusqu'à leur insertion au ganglion abdominal. Cliacum de ces filets fournit vers son tiers postérieur un nerf récurrent assez grand. Dans ce sexe, il part aussi de la partie postérieure du ganglion thoracique, à droite et à gauche du cordon interganglionnaire, un long filet nerveux, non rameux, qui va s'insérer au ganglion de l'abdomen, et qui n'a pas son analogue dans le mâle.

Le ganglion abdominal est arrondi, plus petit que le thoracique : dans le mâle, il est situé avant le milieu de l'abdomen; dans la femelle, tout à fait au bout de celui-ci, à la hauteur de l'origine de l'oviducte. Un coup d'œil comparatif jeté sur les figures de ces deux systèmes nerveux me dispensera de plus de

11. 
détails. J'avoue que je n'ai point des idées arrêtées sur les causes ou les motifs de ces dissemblances dẻ l'appareil nerveux dans les sexes.

Dans la famille des OListrides et dans celle des Muscides calyptérées, que j'ai composéc provisoirement avec celte immense nation des Muscides créophiles et anthomizydes de M. Macquart, les centres nerveux se réduisent au cerveau et à un seul ganglion rachidien. Ce dernier est, dans l'OEstrus, plus oblong que dans les véritables Muscides, et il offre en arrière comme le vestige d'un autre ganglion soudé, terminé par un cordon simple assez long.

Après la description et l'iconographie que j’ai données du syslème norveux des trois morphoses (larve, nymphe et insecte ailé) de la sarcophage, dans un travail dont l'Académie a daigné roter l'admission dans ses mémoires, je craindrais de surcharger la science en reproduisant ici ces détails. Pour ne pas me dévier du plan adopté, pour ne point laisser de lacune, je me bornerai, en choisissant comme objet de comparaison ct de contrôle, in autre type dans le même groupe des Muscides calyptérées, la Calliphora vomitoria on mouche bleue de la viande, à tracer rapidement son appareil sensitif.

Les hémisphères cérébraux, lorsqu'on les étudie étalés, s'épanouissent chacun en une masse optique subréniforme couronnée par la rétine oculaire et sa choroüde. Le bord antérieur du plancher inférieur du cerveau a deux petits mamelons qui émettent les deux nerfs buccaux, tandis que les nerfs antennaires maissent au-dessous de ces mamelons. Le nerf ocellaire est simple, mais renflé à son extrémité, qui laisse apercevoir les trois choroïdes des ocelles, sessiles en apparence, mais où une autopsie heureuse ma permis de distinguer trois courts pédicelles nervenx. .

La partie postérieure du cerveau, qu'on serait tenté d'appeler cervelet ou bulbe rachidien, est percée d'une fente oblongue longitudinale pour le collier asophagien. Le cordon simple qui l'unit au ganglion rachidien émet trois petites paires de nerfs. 
Ce dernier ganglion est unique, grand, ovalaire, thoracique. De ses côtés partent, comme à l'ordinaire, les trois paires de nerfs cruraux, sans compter plusieurs autres d'un petit calibre. Il se continue en arrière en un nerf médian grêle et long, d'où partent des paires symétriques de nerfs digestifs (cinq), et il se bifurque en deux grands nerfs génitaux.

Le système nerveux des Muscides acalyptérées n'oftre pas, dans les diverses peuplades de ce groupe; cette conformité de composition ganglionnaire observée dans les calyptérées. Nous venons de voir dans celles-ci un ganglion unique, et il est thoracique; tandis que, parmi les acalyptérées disséquées jusqu'à co jour, j’ai trouvé tantôt trois de ces ganglions (Ortalis), lantôt deux (Telanocera, Loxocera, Platystoma), tantôt, enfin, et c'est l'immense majorité, un seul. J'avoue que cette dissemblance de composition dans un appareil de première importance organique ćbranle fortement mes convictions sur la légitimité de ce groupe, qu'il faudra, sans doute, diviser en plusieurs familles diversement combinées.

Je borne à ces quelques lignes ce qui concerne l'appareil sensitif des Muscides acalyptérécs. Toutefois, je ne saurais passer sous silence un fait anatomique du plus piquant intérêt fourni par l'hippobosque, un des derniers genres de tout l'ordre des Diptères : je crois ce fait applicable à la généralité des insectes. Les paires de nerfs qui partent du ganglion rachidien unique de l'hippolosque sont disposées sur deux plans : l'un dorsal, l'autre ventral. Cette disposition porterait à penser que de ces nerfs les uns président au mouvement et les autres au sentiment, comme cela existe dans les nerfs rachidiens des animaux le plus haut placés. 


\section{CHAPITRE II.}

APPAREIL RESPIRATOIRE.

Dans les Diptères, comme dans tous les insectes à trachées, l'appareil respiratoire semble cumuler deux fonctions, la respiration el la circulation. Toute expression dubitative doit même être exclue, et l'on peut affirmer que le mode de distribution de cet appareil, qui se divise et se subdivise i l'infini, comme les vaisseaux sanguins des vertébrés, rend incompatible une prétenthe circulation de liguide arec une positive circulation l'air: celle-ci annule par le fait la première.

10 Stigmates. - Il y en a le plus souvent deux paires thoraciques : l'une, mésothoracique, siluée au-dessous de l'angle antérieur du thorax, ayant l'ouverture presque perpendiculaire à l'axe du corps; l'autre, mélathoracique, occupant un sinus tégumentaire au-dessus du trochanter postérieur et ayant l'ouverture trèsoblique. Ces stigmates sont ordinairement oblongs, grands, à deux valves taillées en biscau pour se recouvrir mutuellement dans l'acte respiratoire. Ces valves sont glabres sur leurs bords dans les Tipulaires, garnies de cils fournis ou de franges dans les Tabaniens; ces stigmates sont ronds et comme operculés dans l'Echinomyia, tomenteux dans le Calliphora. La famille des Pupipares, la dernière de l'ordre, offre des singularités pour le nombre de ces stigmates thoraciques: le mélophage, insecte aptère, en a deux paires, et l'hippobosque, ainsi que l'ornithomye. insectes ailés, n'en ont qu'une. On peut voir dans mon travail spécial sur l'anatomie des Pupipares ${ }^{1}$ l'explication que j’ai donnée de ces différents cas. Elle est déduite des habitudes et du genre de vie de ces divers genres de Diptères.

Les stigmates abdominaux sont établis, les uns sur le segment dorsal lui-même, les autres sur la membrane souple qui sépare les segments dorsaux des ventraux: de là leur division en stig-

${ }^{3}$ Études anal, et physiol. sur les Pupipares. (Annales des Sc, nat. $3^{\circ}$ série, $\mathrm{t}$ III; 18.15.) 
mates segmentaires et intersegmentaires. Cette division, aussi importante que naturelle, est applicable aux stigmates abdominaux des insectes des autres ordres. Ces orifices respiratoires, toujours disposés par paires symétriques sur les côtés de l'abdomen, diffèrent aussi par leur nombre suivant les familles : ceux des Culicicles sont intersegmentaires et au nombre de six, en points ronds. Je n'ai pas découvert, non plus que Réaumur, les stigmates abdominaux des Tipulaires: j'en appelle à de nouvelles explorations. Ils sont pareillement intersegmentaires dans les Tabaniens, Asiliques, Syrphides, mais au nombre de cinq dans les premiers et les dernicrs, de six dans les seconds, Ceux des Muscides calyptérées sont segmentaires, au nombre de cinq petits et ronds, nichés au milieu des poils du tégument. Le premier est fort difficile à découvrir, parce qu'il est placé sur un segment rudimentaire de la base de l'abdomen, et il m'a fallu violer la perspective dans le dessin pour le mettre en évidence. Parmi les Muscides acalyptérées, le Platystoma les a segmentaires, et ils m'ont semblé au nombre de trois paires seulement, ce qui est fort extraordinaire. Les deux premières sont semblables à de petits points noirs; la troisième, située près de l'oviscapte, est grande, oblongue; ellipsoïdale. Dans le Nemopoda, genre très-voisin dụ précédent, les stigmates abdominaux sont intersegmentaires et au nombre de cinq paires. Parmi les Pupipares, l'hippobosque n'a que cinq paires de stigmates abdominaux nichés sur le tégument; il y en a sept dans le mélophage.

$2^{0}$ Trachées. J'ai étudié avec un soin scrupuleux leurs diverses formes dans toutes les espèces soumises à mon scalpel, afin de les faire concorder, soit avec la classification, soit avec les autres appareils organiques. En faisant dans mes dossiers d'observations le relevé statistique de ces formes, j’ai été surpris des résultats curieux et inespérés que j'ai obtenus.

Avant d'exposer ceux-ci, il est bon de dire que les Diptères ont les deux ordres de trachées qui se rencontrent en général dans tous les insectes, savoir : les tubulaires ou élastiques, dont l'existence est constante et que je ne m'atlacherai pas à décrire, 
el les vésiculaires ou membraneuses, (qui ne sont pas indispensables. Ces dernières servent exclusivement au vol, et l'animal, suivant les Jesoins de cet excrcice aérien, peut ì volonté les cnller à divers degrés.

Je distingue, dans les trachées vésiculaires, trois formes particulières : $1^{\circ}$ les ballons ou aérostats, vastes réservoirs logés à la base de la cavité abdominale, s'anastomosant d'une part avec les utricules thoraciques, de l'autre avec les canaux bronchiques ou les grandes trachées latérales. Le plus ordinairement, il n'y en a qu'une paire, et quclquefois ils manquent entièrement; $2^{\circ}$ les utricules thoraciques, réservoirs de moyenne grandeur, parfois mème très-petits, mais ne manquant presque jamais; $3^{\circ}$ les bulles cíphaliques, vésicules d'une pelitesse extrême et prodigieusement multipliées dans le crâne, où, en même temps qu'elles servent d'édredon au cerveau, elles facilitent, en diminuant la pesanteu de la tête, la direction des mouvements généraux.

En parcourant la série des familles, je signalerai celles qui sont pourvues ou privées de ballons trachéens. Nous trouverons des faits piquants d'une explication parfois embarrassante.

Il existe une paire de ces ballons dans les Culicides, les TipuIaires et les Tabaniens. On connaît le sifflement aigu des premiers et le bourdonnement nourri des derniers. Quant aux Tipulaires, dont le vol est peu bruyant, mais assez actif après le coucher du soleil, leurs ballons sont aussi beaucoup plus petits. Dans la famille des Strationydes, l'Ephippium et les Stratiomys ont deux aćrostats, tandis que les Sargus, Chrysomyia, Vappo, qui terminent ce groupe, n'en ont pas; mais les allures paisibles et le vol silencicux de ces trois derniers genres justifient cette privation. Les $\Lambda$ siliques, chasseurs robustes qui se précipitent comme un trait sur leur proie, qu'ils entraînent dans les airs, ont tous deux ballons, et les Empides, leurs voisins, n'en ont pas, tandis que le Cyrtus, qui succède ì ces derniers, en est pourvu. Et que penser de l'absenec complète des aérostats dans les Bombyliers, dont jai dissćqué sept espèces? Comprenez-vous une privation aussi absolue 
dans des insectes dont la vie si agitée est toujours aćrienne, et dont le bourdonnement aigu, origine de leur dénomination, est susceptible sous un soleil ardent de toutes les modulations? La nature ne nous doit pas compte de ses infractions à nos lois. Passons outre et déclinons encore notre compétence devant les Anthraciens, Diptères tout aussi bien aéricoles que les précédents mais bien moins vifs et nullement bourdonnants, qui, cependant, portent dans leurs flancs deux grands ballons arrondis. Les Thérévides, prompts au vol et danseurs aériens, en ont aussi deux, et les Leptides, qui les suivent, en sont dépourvus. Les Dolichopodes, aussi rapides à la marche qu'au vol, et les brillants Syrphides, qui partagent leur existence entre la corolle qu'ils eflleurent, et leurs danses amoureuses, leurs équilibres aériens, sont munis d'aérostats parfaitement conditionnés. Le Scenopinus, jeté par l'imperfection de nos méthodes entre deux grandes nations de Diptères aérostatiques, vient témoigner de la privation des locomotives atmosphériques par ses habitudes sédentaires, sa marche lente et monotone, son peu d'aptitude à mettre en exercice des ailes toujours ployées et comme collées sur son corps. Les OEstrides, remarquables par le bourdonnement aigu et la prestesse du vol, et cette longue série des Muscides calyptérées, des Dexia, Echynomyia Musca, Lucilia, Anthomyia, ctc. tous Diptères essentiellement actifs, turbulents et bruyants dans leurs exercices aériens, ont une paire de ballons : je l'ai vérifié sur quarante et une espèces. La catégoric non moins populeuse des Muscides acalyptérées, depuis le Sepedon jusqu'au Splcerocera, de ces petites mouches qui habitent ou les rivages solitaires, ou les plantes marécageuses, ou les lieux ombragés, qui ont une démarche grave et compassée, un vol paisible et muet; ces Diptères, dis-je, dont j’ai disséqué quarante-six espèces, sont tous, sans exception, déshérités d'aérostats comme de cueillerons aux balanciers. Enfin, l'Hippobosca, qui termine la chaîne diptérologique, confirme l'absence de ballons par son inhabileté à un vol soutenu et par sa vie parasite passée dans les régions les plus abritées du cheval. 
APPAREIL RESPIRATOIRE DES LARVES.

Pour compléter, autant que le permettent nos connaissances actuelles, ce qui concerne l'appareil respiratoire des Diptères, je vais exposer succinctement mes recherches sur celui des larves - de ces insectes en prenant pour types de ces organes de la respiration ceux qui offrent des combinaisons diverses fournies par le nombre des stigmates. Ce nombre, jusqu'à présent, se borne à une, à deux ou à huit paires.

Dans la famille des Tipulaires, on trouve les deux extrêmes de la combinaison. Dans les larves hémicéphalées terricoles, il n'y a qu'une seule paire de stigmates, et il y en a huit dans les fongirores.

Cies stigmates, dans la larve terricole du Tipula lunata, sont postérieurs et logés dans la caverne stigmatique du bout de l'abdomen. Ils se présentent au dehors sous l'aspect de deux plaques orbiculaires assez grandes, rapprochées, noires, avec un limbe moins foncé. Quelles qu'aient été et l'inspection la plus scrupuleuse et mes expérimentations sur la larve tranquille ou violentée, a sec ou immergée, je n'ai jamais pu saisir le moindre mouvement qui pût se rapporter au jeu, au mécanisme de la respiration. Après avoir isolé le stigmate, après avoir soigneusement râclé le pigment qui forme la couleur noire du disque, après avoir, dans une circonstance, détaché avec bonheur un grand lambeau de ce pigment sans offenser la trame sous-jacente, je l'ai soumis à la plus puissante lentille de mon microscope et j'ai cru y reconnaître de petits points ou des trous disposés sans ordre, de manière que je comparais cette membrane à un crible. Quant au limbe moins foncé, on y reconnait de fines lignes transversales subgéminées sur un fond presque diaphane. Cette dernière texture rappelle celle, plus facile ì constater, des stigmates en fer à cheval des larves de Coléoptères lamellicornes (Eclonia, Oryctes).

Les trachées de notre larve de Tipule forment, par leur en- 
semble, un système vasculaire complet, d'une parfaite symétrie, et établissant ainsi, non-seulement une circulation, mais presque une double circulation aérifère. Les canaux bronchiques latéraux sinsèrent au centre des stigmates et conservent le même calibre jusqu'à la partie antérieure du corps; là, ils s'atténuent pour s'anastomoser entre eux, soit par des arcades, soit par des conduits traversiers antérieurs ou postérieurs. Dans leur trajet, les canaux bronchiques plus ou moins sinueux fournissent des trachées nutritives assez symétriques: la figure dira le reste. Toutefois, je décrirai en peu de mots le petit systéme trachéen qui revêt la face interne des stigmates. Il y a à celle-ci une houppe orbiculaire, une sorte de parenchyme formé par une immense quantité (des centaines) de trachéoles blanches bien nacrées, d'une finesse qui surpasse celle du brin le plus délié de la soie, et dont le micror scope met en évidence les subtiles ranifications. En déchirant cette houppe, cette curieuse ébauche de poumon, jai bien aperçu les souches trachéennes où elle prend sans doute naissance; mais je n'ai pas constaté son mode de connexion avec le stigmate.

Les larves céphalées fongivores des Tipulaires (Mycetophila inermis) ont huit paires de stigmates sous la forme de très-petits points noirs situés à nu sur les côtés du corps, savoir : une thoracique, plus grande au premier segment après la țête, et sept abdominales aux sept segments qui suivent le troisième. Les canaux bronchiques paraissent naître directement des stigmates thoraciques et règnent parallèlement de chaque côté de la région dorsale en émettant un grand nombre de branches nutritives. Elles reçoivent de chaque stigmate abdominal un conduit simple et court, et communiquent ensemble par autant de canaux traversiers (qu'il $y$ a de segments.

Quel système circulatoire trouverez-vous plus symétrique, plus élégant, plus parfait que celui-là? Un regard sụr son portrait suppléera à une description détaillée. Voyez comme ces nombreux canaux traversiers sont aptes à favoriser la circulation de l'air et à obvier aux embarras que les vicissitudes de la vie 
pourraient faire éprouver à l'un ou à l'autre des canaix bronchiques!

Si dans la larve terricole où l'appareil trachéen se réduil à une seule anse continue dont les bouts sont les deux stigmates, l'inspiration et l'expiration ont évidemment licu par ceux-ci, le grand nombre des orifices respiratoires de la larve fongivore peut nous laisser des doutes sur ce point. Peut-être bien que c'est par les stigmates antérieurs, qui sont les plus grands, qu'a lieu la prise d'air el que sa sortie s'effectue par les stigmates abdominaux.

Les larves acéphalées des Muscides ont deux paires de stigmates: l'une, postérieure, simple; l'autre, antérieure, à plusieurs digitations el a plusicurs ostioles respiratoires. Dans un mémoirc qui est encore entre les mains de l'Ácadémie, qui traite el de la prétendue circulation et des métamorphoses organiques dans les irois états de la mouche vivipare ou sarcophage, jai fait connaître le système respiratoire de la larve de celle-ci : ce sont les stigmates postéricurs qui inspirent l'air, et les antérieurs qui l'expirent.

Dans les trois formes différentes de respiration que je.viens de signaler, le phénomène plıysiologique est toujours le même.

\section{CHAPITRE III.}

IPPAREIL DIGESTIF FN GÉNÉRAL:

Les Diptères, par la structure de leur Jouche, sont destinés : poimper, à sucer, à lécher un aliment liquicle ou pulvérulent. Quelques-uns d'entre eux, comme Asilus, Scatophaga, etc. peuvent saisir une proie vivante, la déchirer, la broyer, soit avec leurs griffes, soit avec quelques parties de leur bouche, et la disposer ainsi à être sucée ou avaléc. On appelle sucoir ou trompe leur bouche. Ce suçoir a des formes une composition qui varient depuis celle du Bambylius, qui, toujours en évidence et inoffensive, a la longueur du corps, jusqu’à celle dı Tabanus, qui est un ins- 
trument vulnérant, et à celle du Mlusca, propre à lécher, rétractile et invisible dans le repos.

L'appareil de la digestion se compose des glandes salivaires et du tube alimentaire avec ses annexes.

Les glandes salivaires existent dans tous les Diptères et sont toujours simples, c'est-ì-dire formées pour chaque côté par um seul vaisseau ou boyau blanchâtre ou diaphane, suivant le degré d'élaboration de la salive, tantôt plus ou moins long et capillaire, flexueux, reployé ou pelotonné, tantôt en bourse ovalaire nu oblongue. Ces vaisseaux sont sécréleurs par leurs parois, réservoirs par leur cavité. Ils aboutissent en avant à un col efférent, et les deux cols se confondent dans la tête en un seul conduit excréteur capillaire, qui verse dans la bouche le produit de ta sécrétion. Cette composition, cette explication, sont communes à tout l'ordre.

Le tube alimentairc présente, relativement à son étendue, de curieuses différences depuis le Culex, où il n'a que la longueu du corps jusqu'à l'Ilippobosca, où cette longueur a huit à neuf fois celle de l'insecte. Cette progression croissante à mesure que l'organisation est moins élevée est un fait aussi piquant que rigoureusement établi. Les contenta de ce tube peuvent éclairer sur le genre de nourriture des insectes.

Cet organe se compose, en suivant l'ordre de leur position, de l'msophage, de la panse, du ventricule chylifique, des vaisseaux hépaliques et de l'intestin. Dans quelques espèces, il est aussi le siége d'une glande odorifique (Sepsidées).

L'osophage est, en général, "fort court et d'une grande ténuité. Je me dispenserai de le mentionner désormais dans l'histoire des familles, à moins qu'il ne présente quelque particularité.

La panse, qui, à quelques exceptions près (Asilus, Pupipares), ne manque jamais dans les Diptères, est constamment placée au côté gauche du tube digestif, l'insecte étant posé, quant à l'œeil de l'observateur, dans l'attitude de la marche en avant. On distingue à cet organe : $1^{\circ}$ un col tubuleux et grêle, inséré à la 
terminaison de l'osophage; $2^{\circ}$ un réservoir, le plus souvent logé a la base de la cavité abdominale, dont la configuration est tantồt simplement ovoïde, tantôt, et c'est le plus ordinaire, bilobée ou en bissac. Cet organe, ainsi que le prouve son insertion avant l'origine du ventricule chylifique, est le premier réceptacle de l'aliment et parait favorable à la rumination.

Le ventricule chylifique forme la plus grande longueur du canal alimentaire, et c'est lui qui se reploie en circonvolutions. C'est dans cet organe que l'aliment mélangé, combiné avec la bile, reçoit les conditions qui le rendent propre à l'assimilation. Son orifice a, dans les grandes divisions de l'ordre, une configuration et une structure fort différentes. Ainsi, il est simple dans quelques Tipulaires et dans les derniers genres des Diptères. Il s'accompagne dans les Tabaniens, Asiliques, Stratiomydes, Bombyliers, Rhagionides, Dolichopodes, Scénopiens, de deux bourses ventriculaires simples. Ces bourses ne sont que des prolongements latéraux, des boursouflures régulières et permanentes des parois de l'organe; elles sont destinées au séjour, à l'élaboration de la bouillie alimentaire : elles sont doubles ou appendiculées dans tous les Syrphides. L'orifice du ventricule est formé d'un godet ou bourrelet orbiculaire et ombiliqué, dans les Conopsaires et les deux familles des Muscides.

Les vaisseaux hépatiques ou biliaires, presque toujours au nombre de quatre, rarement de cinq (Culex), ont leurs extrémités flottantes, excepté dans les grandẹs Tipulaires, où ils forment deux anses très-reployées, mais à quatre insertions. Ils ne s'insèrent jamais au rectum, en sorte que leurs fonctions ne sauraient être ambiguës; mais à l'extrémité postérieure du ventricule chylifique, quelquefois par quatre points isolés, le plus souvent par deux canaux cholédoques, dont chacun est l'aboutissant de deux vaisseaux, rarement par un seul canal commun aux quatre vaisscaux (Stratiomydes).

L'intestin, distinct du ventricule chylifique par une valvule qui correspond à l'iléo-cœcale des grands animaux, est d'abord grêle et filiforme. Avant de se terminer à l'anus, il se renfle en un rec- 
tum ovale ou oblong où se voient ordinairement quatre boutons charnus ou orbiculaires ou conoïdes. Ces boutons, au moins dans plusieurs Diptéres (les Pupipares surtout), sont des muscles papil. liformes, dont la base est fixée aux parois de l'organe et visible à l'extérieur, tandis que le reste est comme pendant dans la carite du rectum; ils ne sont pas étrangers à la défécation.

\section{CHAPITRE IV.}

APPAREIL GÉNITAL EN GÉNÉRAL.

1' Appareil génilal mále. - Il se compose, comme dans les insectes des autres ordres, et même comme dans les animaux en général, de testicules, de conduits déférents, de vésicules séminales, du canal éjaculateur et de la verge, qui est renfermée dans l'armure copulatrice.

Les testicules, placés vers la fin de la cavité abdominale, sont des organes binaires, et chacun d'eux est toujours simple, c'est-àdire unicapsulaire; ils sont libres, indépendants l'un de l'autre, excepté dans quelques Asiliques, où les deux sont renfermés dans une enveloppe commune accessoire, un véritable scrolum. Leur configuration varie à l'infini depuis l'ovalaire ou l'oblongue jusqu'à la filiforme, plus ou moins enroulée ou agglomérée; leur couleur est blanchâtre dans les Tipulaires, Tabaniens, etc. d'un brun plus ou moins intense à l'extéricur dans les Asiliques, Muscides, etc.

Les conduits déférents, le plus souvent grêles comme un fil, ont une longueur différente suivant les genres; quelquefois, on ne les distingue pas du testicule, dont ils ne semblent que la continuation. Ils sont parfois d'une extrême brièveté et presque nuls. Ce n'est que fort rarement qu'on leur trouve des renflements ou des replis particuliers qui simulent un épididyme.

Il n'existe ordinairement qu'une seule paire de vésicules séminales. Les conduits déférents s'y insèrent immédiatement avant qu'elles confluent pour la formation du canal éjaculateur. Dans 
quelques Muscides acalyptérées, ces vésicules sont doubles de chaque côté, et dans plusicurs genres des calyptérées (Musca, Curtoncura, etc.) elles n'existent pas du tout. Leur forme se diversifie suivant les familles, depuis celle d'un réservoir ovalaire jusqu'à cclle d'un filet tubuleux, dont la longueur, tantôt égale ì peine celle du testicule, et tantôt se reploie en agglomérations inextricalıles; quelquefois, il existe une de ces vésicules impaire.

Le canal éjaculaleur, ou le tronc commun de tout l'appareil sécréleur et conservateur du sperme, a aussi ses diversités de formes. Il est parfois excessivement court (Culicides), et dans d'au tres familles, fort long et reployé. Dans les Syrphides, il offre, comme un trait propre à cette famille, un réservoir spermatique. Dans les espéces où les vésicules séminales manquent (Mlusca, elc.). sa longueur et ses flexuosités les remplacent.

L'armure copulatrice, réceptacle de la verge, est une machine des plus compliquées, destinée à se porter hors du corps lors do l'union des sexes. Les nombreuses pièces plus ou moins symétriques, cornées ou coriacées qui la composent, combinent leur action, soit entre elles, soit avec les organes externes de la femelle pour consommer l'acte de la fécondation. Elle varie suivant les espèces et constitue, par sa configuration comme par sa structure, la garantie de l'inviolabilité des types spécifiques.

$2^{\circ}$ Appareil génilal femelle. - Les organes qui entrent dans sa composition sont: les ovaires, l'oviducte, la glande sébifıue avec les réservoirs séminaux, les œufs et l'oviscapte.

Les ovaires sont constitués chacun par un faisceau de gaînes ovigères courtes ou longues, en nombre déterminable ou innombrable, uni ou pluriloculaires; leur étude peut déjà décider de l'abondance de la progéniture. L'ovaire a un calice ou central, ou inférieur ou postérieur, où les œufs à terme peuvent s'accumuler pour être au Jjesoin transmis par un col à l'oviducte. Dans les Diptères vivipares, les oufs passent des ovaires dans des réservoirs particulicrs ou ils subissent une incubation et une éclosion, de manière que ces réservoirs peuvent renfermer à la fois et des 
aufs et des larves : je les ai appelés pour cela ovo-larvigéres. Il est aussi des Diptères qui ne mettent au monde ni oufs ni larves, mais des chrysalides ou pupes : ce sont les Pupipares. Dans ce mode singulier de parturition, la mère ne donne le jour qu’à une seule chrysalide.

L'oviducte est le tronc commun des ovaires, comme le canal éjaculateur est celui des organes spermifiques; il présente de nombreuses modifications de longueur et de structure. Dans les Muscides vivipares, il se développe pour devenir le réceptacle des oufs et des larves; son orifice extérieur est. la vulve, et s'accompagne le plus souvent de deux appendices unis ou biarticulés que j’appelle les tentacules vulvaires. Ils ont pour fonction ou de servir de grandes lèvres lors du coït, ou de diriger, de colloquer les œufs au moment de la ponte.

Mais l'oviducte ne sert pas seulement à l'acte copulateur comme un vagin et à éconduire le produit de la gestation. Les oufs it terme, c'est-à-dire parvenus à dernier degré de leur croissance, n'ont pas encore reçu dans leurs gaînes ovigères l'imprégnation prolifique, quoiqu’ils aient sans doute été mis en éveil par la commotion coïtale. A leur trajet dans l'oviducte, ils doivent recevoir d'un ensemble d'organes inséré sur celui-ci l'ablution séminale et un enduit conservateur. Depuis plus de vingt ans, j'avais donné le nom de glande sébifique à cet ensemble d'organes, dont les uns sont évidemment sécréteurs et les autres simplement réservoirs. Aujourd'hui, qu'une étude plus approfondie de cet appareil complexe et que les découvertes importantes de Von Siebold et M. Loew sont venues, sinon déchirer, du moins soulever le voile de ses attributions physiologiques, il convient de modifier une dénomination dont la signification est trop restreinte et trop partielle. Gelle d'appendices de l'oviducte de M. Loew me semble peu physiologique, et jadopterai, au moins provisoirement, la double désignation de réservoir séminal et de glande sébifique.

Ces deux organes ont une forme, une composition fort variables, suivant les familles, et je réserve pour l'étude anatomique de ces 
familles l'exposition de ces curieuses différences; je me bornerai actuellement à indiquer les fonctions respectives et la nomenclature des diverses parties de cet appareil. Quoique les auteurs précités aient diminué les incertitudes physiologiques sur ces organes, ils sont loin d'avoir entièrement dissipé les miennes, ainsi qu'on le verra dans l'anatomie des familles.

Les organes dont il est question ont, pour la plupart, leur insertion à la paroi supérieure de l'oviducte et à la moitié antérieure de ce conduit. Le réservoir séminal précède presque toujours la glande sébifique, et cette position respective est conséquente aux attributions physiologiques de ces deux organes. Sil est vrai, comme je le crois, que les œufs, au moment d'être pondus, sont enduits par un vernis conservateur fourni par la glande sébifique, il est de toute rigueur qu'auparavant ils soient fécondés par lc baptême prolifique du réservoir séminal.

Mais il existe entre mes dénominations techniques et celles de M. Loew une grave et singulière dissemblance, qui tient à la manière d'envisager les fonctions des organes. Ce savant appelle glande du mucus mon réservoir séminal, et receptaculum scminis ma glande sébifique. Il est tombé, je crois, dans un véritable quiproquo physiologique, et c'est en renversant les rôles qu'on arrive au viai.

Quoi qu'il en soit, je désigne sous le nom de réservoir séminal un organe presque toujours binaire ou pair, composé de deux bourses semblables, de conliguration très-diverse, inséré en avani de la glande sébifique, et quelquefois muni d'un col commun fort court.

La glande sébifıque consiste, dans le plus grand nombre des Diptères, en trois vésicules suborbiculaires à centre plus ou moins noir, éminemment sécrétoires, que je nomme orbicelles et M. Loew capsules glanduliformes; ces orbicelles sont, en général, munis d'un conduit efférent subcapillaire, droit ou fléchi, on flexueux ou roulé sur lui-même. Je ne comprends pas comment M. Loew, après avoir reconnu dans cet organe tous les traits 
propres à un appareil sécréteur, à une glande, a pu se laisser entrainer à la dénomination si insignificative du receptaculum seminis.

Les cufs des Diptères sont ou ovales, ou oblongs ou allongés et parfois hémisphériques; la plupart sont blancs ou jaunàtres, mais il y en a de noirs comme du charbon (Tipules).

L'oviscapte est un organe destiné à introduire, lors de la ponte, les œufs dans un milieu plus ou moins résistant; il varie singulièrement suivant les familles; quelquefois, il consiste en un instrument corné et il deux lames, toujours en évidence au bout de l'abdomen (Tipulaires); dans un très-grand nombre de Diptères, il se compose de plusieurs tulres rentrant les uns dans les autres, comme les tuyaux d'une lunette d'approche, et leur ensemble est rétractile au gré de l'insecte.

\section{CHAPITRE V,}

TISSU ADIPEUX SPLANCHNIQUE.

Dans mes diverses publications entomotomiques, j’ai presque donné l'importance physiologique d'un organe à ce tissu; il remplit, en effet, une fonction nutritive secondaire, en même temps que, peut-être, il contribue à concentrer autour des viscères la chaleur vitale. On sait que son abondance est en raison inverse de l'activité du genre de vie.

Ce tissu existe en proportions variables dans les Diptères; mes nombreuses autopsies m'ont fourni, à ma vive surprise, des faits très-piquants sur sa quantité et sa nature suivant les habitudes des espèces. On conçoit quel soin scrupuleux il faut apporter pour recueillir avec exactitude les résultats si faiblement nuancés de semblables dissections; mais ces nuances n'en sont pas moins des vérités.

Les Culicides, inșectes qui fuient la lumière du soleil et qui sont exposés à des jeûnes plus ou moins prolongés ont, sous les viscères, une couche adipeuse grisâtre, assez fournie. Les Tipu-

$$
11 \text {. }
$$


laires, qui marchent peu et volent mal, ont, dans les grandes espèces, une pulpe graisseuse, blanchâtre, formée de sachets ovalaires ou parfois de lambeaux éguenillés, grêles, entre-croisés, et enlaçant les viscères : cette pulpe est presque nulle dans le Coroplatus. Ce ne sont que des granules libres, mais abondants dans les Macrocera, Sciara, Mycetophila. Le Rhyphus a une grande quantité de sachets oblongs. La graisse est moins considérable dans le Bibio, insecte plus diurne que les autres. Les Psychoda, qui vivent dans les réduits obscurs des fossés, ont de nombreuses granulations ovales ou arrondies.

Les larves des Tipulaires terricoles, animaux voraces, aveugles et cachés dans les entrailles du sol, sont pourvues d'une assez grande abondance de tissu adipeux : celui-ci se présente sous la forme de nappes ou de larges tabliers épiploïques blanchâtres, criblés de trous arrondis ou de réticulations comme une dentelle. Dans la larve du Xyphura, ce sont des lanières fort étroites, croisées en mailles lâches et irrégulières; celle du Ceroplatus a des lambeaux grisâtres, filiformes, flottants; celle du Mycetophila a de longs rubans déchiquetés oủ les granules sont disposés sur un même plan.

Dans les Tabaniens, dont les femelles sanguinaires ont une vie très-active sous un soleil ardent, mais qui, dans les temps couverts et frais, sont retirées et souvent forcées à un régime austère, on trouve constamment autour du ventricule chylifique un grand châle épiploïque blanchâtre, formé de sachets enchevêtrés de trachéoles, et indépendamment de cela, si la dissection est heureuse, une membrane hyaline péritonéale, plus ou moins collée contre les parois abelominales. Parmi les Stratiomydes, l'Ephippium, de mœurs assez paisibles, a des granules adipeux libres et des sachets grands assez fournis, tandis que les Stratiomys, plus actifs, n'ont presque pas de réserve graisseuse. Le Vappo et le Beris, assez sédentaires, ont, au-dessous des viscères, des granules détachés assez grands. Dans les Sargus, qui n'ont pas beaucoup de vivacité, on trouve des sachets polymorphes abondants, formant avec les 
trachées des espèces de grappes ou de guirlandes. Le Cyrtus a des granules libres nombreux; les Empis, assez volages, ont à peine quelques rares lambeaux d'une graisse jaunâtre; les Asiliques, chasseurs vigilants, le plus souvent en faction, d'un vol brusque, rapide, mais non prolongé, ont le châle ventriculaire des Tabaniens et de larges nappes sous-viscérales recouvertes de sachets. Les Anthraciens et les Bombyliers, dont l'existence aérienne est plus ou moins agitée, ont sous les viscères quelques lambeaux de nappes graisseuses; mais les premiers, incontestablement moins vifs, ont des granules détachés qui ne s'observent pas dans les seconds. Ces nuances ne sont futiles qu'en apparence. Les Dolichopodes, les Thérévides, les Rhagionides, tous Diptères d'une vie assez active, ont à peine quelques follicules polymorphes et rarement des granules libres; les Syrphides, presque toujours suspendus en l'air et bourdonnants, ont quelques guenilles adipeuses rares; le Sccnopinas, au contraire, d'un caractère morose et d'habitudes sédentaires, a abondamment une pulpe granuleuse blanche et des grains détachés, les deux formes du tissu adipeux qui annoncent par leur réunion les propriétés vitales les moins énergiques. Nous ne connaissons pas hien les habitudes des Conopsaires; mais à en juger par l'abondance des granules adipeux pulvériformes, ils ne doivent pas être fort actifs. Les OEstrides m'ont offert dans le Cephalemyia une enveloppe péritonéale aranéeuse, dans l'OEstrus quelques granules libres : je parierais que le premier a une vie plus agitée. Les Muscides calyptérées, qui, en général, se font remarquer et par la prestesse de leur vol bourdonnant et par la rapidité de leur marche, ont une pulpe adipeuse médiocrement abondante sous la forme ou de granules libres, ou d'une couche pariétale, ou de grumeaux; mais leur graisse est toujours blanche ou grise. Les Muscides acalyptérées, oủ les espèces sont presque toutes d'unc humeur paisible et sédentaire, peu habiles au vol et à la course, ont une quantité assez considérable de tissu adipeux. Mes procès-verbaux de dissection m'ont donné des résultats singuliers. Les espèces des Sepedon, Tetanocera, Loxocera, 
genres riverains ou habitants des plantes marécageuses, ont audessous des viscères une couche pariétale d'une graisse fauve, chocolat ou blonde, et, outre cela, une pulpe grumeleuse blanchâtre située sur les organes; les Scatophaga et les Sapromyza, dont le genre de vie est bien différent, ont des flocons adipeux uniformément blancs; les Helomyza, amis de l'ombre des forêts, mais non riverains, ont des lambeaux graisseux et des granules libres; le Tephrilis, que j'ai souvent rencontré dans les bois pondant ses œuf's sur les champignons parasites, a sous les viscères une couche pariétale chocolat, quoique l'espèce ne soit pas riveraine: sa vie privée est encore à étudier. Le Plalystoma, habitant grave et sédentaire des troncs d'arbres ombragés, a une abondance singulière de granules adipeux sphériques, libres, błanchàtres, qui rappellent ceux que j’ai signalés dans les Ichneumons et autres Hyménoptères. Le Calobata et l'Ulidia, qui vivent sur les végétaux des bords ombragés des fossés, ont, le premier, une couche sous-viscérale fauve, le second, un ruban de cette coulcur le long des flancs et des granules libres, blancs, pulvériformes. Le Lauxania a le ruban latéral de l'Ulidia, mais blond et sans granules; le Lonchaa, genre contigu au précédent, a une couche sous-viscérale épaisse, blanche, et par-ci par-là quelques lambeaux ferrugineux; l'Ochtera, malgré sa vie toute riveraine, n'a qu'une pulpe adipeuse grisâtre, et le Notiphila, cohabitant du même rivage, a des sachets ovalaires blancs suspendus aux trachées. Je n’ai aperçu dans le Teichomyza, domicilié des écuries, que des lambeaux polymorphes d'une graisse subdiaphane; l' $E$ phydra-ripicole, des lieux ombragés, a une pulpe alsondante chocolat, et aussi quelques sachets gris; le Sphcerocera, qui se plait dans les crottins des forêts, a des grumeaux abondants, blancs, et une couche sous-viscérale roussâtre; enfin, les Phora, agiles à la course et presque cosmopolites, n'ont que des granules libres. Parmi les Pupipares, dernicr groupe de l'ordre, l'Hippobosca a des granules adipeux ronds, souvent contigus en séries moniliformes, et quelques guenilles clair-semées. 


\section{DEUXIENE DIVISION. \\ INATOME PARTICULIÉRE DES FAMILLES.}

\section{FAMILLE DES CULICIDES.}

Par le seul fait de son habitation importune dans nos demeures, le cousin, insecte si frêle, mais si redoutable, est devenu l'objet de l'étude séricuse des plus recommandables auteurs depuis Aristote et Pline, jusqu'à Swammerdam, Leuwenhoeck, Réaumur, de Géer, Latreille, etc. Réaumur, le modèle des observateurs. semble avoir épuisé tout ce qui est relatif aux formes extérieures, aux mours, au genre de vie, aux métamorphoses de ce Diptère; il ne manquait, pour compléter son histoire naturelle, que de porter le scalpel dans ses viscères, que de mettre en évidence les ressorts secrets des divers actes extérieurs : je viens offrir à la science ma part de matériaux pour atteindre ce but.

Les Culicides disséqués se bornent aux suivants :

1. Culex annulaius. Fabr.

2. - lutescens. F.

3. Anopheles bifurcaius. MEIG.

La première espèce étant la moins petite et la plus répandue dans la contrée que j'habite, c'est celle-là dont j’ai plus particulièrement étudié et représenté l'anatomie.

\section{CHAPITRE PREMIER.}

APPAREIL DJGESTIF.

Tout le monde sait que les cousins s'abreuvent du sang des animaux, mais tout le monde ne sait pas que les femelles seules sont sanguinaires. Quelques auteurs avaient émis l'idée quà défaut de sang, ces insectes suçaient les fleurs ou les humeurs des feuilles, et Réaumur l'avait combattue. L'anatomie avait droit de 
résoudre la question. Elle a constaté dans l'estomac des femelles l'existence du sang, et dans celui des mâles un aliment incolore. Ce fait, tout extraordinaire qu'il est, n'est pas isolé en entomologie, et les Tabanus nous en fourniront bientôt un autre exemple.

La trompe longue et menue du cousin se termine par un bouton bilabié et n'est que le fourreau d'un suçoir de cinq pièces sétiformes, dont les deux centrales, munies d'aspérités, font l'office d'instruments vulnérants, de dards, et les autres de lancettes et de sonde cannelée.

Les glandes salivaires des cousins ont une finesse capillaire et atteignent à peine par leur bout libre le tiers postérieur de la cavité thoracique.

J'avoue que je ne vois pas sur quoi se fonde l'opinion de ceux qui avancent que le cousin envenime la piqûre en y insérant un liquide irritant; je ne trouve nulle part, dans l'intérieur de cet insecte, la glande ou l'organe spécial d'un semblable virus. Cette glande n'existe pas davantage dans le stomoxe, le taon et autres Diptères à morsures douloureuses, tandis qu'on la rencontre sous des formes très-variées dans tous les Hyménoptères, qui produisent des piqûres envenimécs au moyen d'aiguillons rétractiles placés à la région de l'anus etrecevant des glandes spéciales vénénifiques une liqueur irritante. La considération de la structure du dard du cousin, dont les dents acérćes sont si favorablement disposées pour déchirer le tissu, et celle du mouvement de succion me paraissent suffisantes pour se rendre raison et de la douleur et de la formation subite d'un exanthème inflammatoire. Il est donc plus conforme aux faits anatomiques, il est plus physiologique de penser que la liqueur salivaire se mêle au sang lors de la morsure comme à tout liquide alimentaire pour en rendre la digestion plus facile.

Le tube alimentaire ne dépasse que peu ou point en longueur celle de l'insecte; il est par conséquent à peu près droit. Dans le Culex lulescens, jai trouvé l'osophage un peu renflé à son insertion ventriculaire. La panse a son réservoir simple, globuleux ou 
ovoïle quand il est plein de liquide; oblong, plissé sur ses bords dans le cas contraire. Je n'y ai jamais rencontré qu'un liquide incolore ou à peine ambré, même lorsque le ventricule chylifique était gorgé de sang. Ce fait semble, au premier abord, inexplicable; cependant, en analysant physiologiquement ce qui se passe lors de la piqûre du cousin, la solution est moins embarrassante. J'avais d'abord pensé que, pendant la déglutition, il se faisait, par une chimie organique encore mal comprise, un déparı de la matière colorante, qui, plus essentiellement nutritive, franchissait seule l'orifice ventriculaire, tandis que le sérum gagnait le réservoir de la panse comme aliment plus grossier pouvant être utilisé dans les temps de diselte; mais l'explication suivante est tout aussi admissible et doit peut-être se combiner avec l'autre. L'insecte, avant d'avoir déterminé par sa piqûre la fluxion sanguine dans les vaisseaux capillaires du tissu cutané, a dû sucer de la lymphe pure, et c'est celle-ci qui est tenue en réserve dans la panse, soit pour être ensuite rejetéc par le vomissement, soit pour servir aux besoins dont j'ai parlé.

Le ventricule chylifique offre constamment à son origine une paire de bourses ventriculaires ovoïdes, plus ou moins pédicellées, et de volume variable, suivant la quantité de liquide ou de bulles d'air qu'elles renferment ${ }^{1}$. Ce liquide est ordinairement ambré. Le ventricule est plus ou moins renflé, en une poche ellipsoïdale, dès qu'il a atteint l'abdomen. On voit parfois à son origine une sorte de renflement qui semble annoncer une tendance à se bilober comme dans les dernières Tipulaires. Je l'ai trouvé ainsi dans le Lutescens. Cet organe, quand il est gorgé outre mesure de sang, prend, aussitôt qu'on a ouvert les parois abdominales, un développement énorme.

1 Suivant M. Pouchet (Compte rendu de l'Acadénie des sciences, octobre 1847), la larve du cousin aurait huit estomacs vésiculifornes. Cet auteur appelle estomacs ce que depuis long. temps j’ai désigné sous le nom de bourses ventriculaires dans les Ortoptères, quelques Névroptères et le cousin ailé lui-même. Ces bourses, plus ou moins verticillées à l'origine du ventricule chylifique, ne sont giu'au nombre de quatre dans les grandes larves des Tipules, comme on va le voir. 
Les vaisseaux hépatiques, courts comparativement à ceux des autres Diptères, sont au nombre de cinq sculement, et ce chiffre impair est fort rare dans les insectes. Cette exception pour les cousins a été constante dans les nombreux individus soumis à mes dissections. Ces vaisseaux se fléchissent en anse pour se diriger toujours en arrière. Ils sont grêles, atténués un peu vers leur origine, tantôt assez uniformément blanchâtres, tantôt diaphanes, avec des mouchetures blanches, qui ne sont que tes flocons intérieurs de la bile coagulée, et qui peuvent en imposer à des yeux peu scrupuleux pour des boursouflures ou des varicosités. Leur bout libre est parfois renflé en massue.

L'intestin, moins long que le ventricule, est d'abord filiforme; puis it se renfle en un rectum ovalaire, où se voient deux paires de boutons charnus orbiculaires.

\section{CHAPITPE II!}

APPAREIL GÉNITAI.

Les Culicides domnent plusieurs générations par année : six ou sept, suivant Réaumur. Leur accouplement se fait en l'air, le soir ou la nuit, ainsi que l'a constaté de Géer. Les femelles déposent a la surface des eaux stagnantes leurs oufs réunis en un petit tas. Les larves éclosent deux ou trois jours après la ponte et sont aquatiques toute leur vie.

\section{AR'TICLE I ${ }^{\circ}$}

APPAREIL GriNital Mile.

Les lesticules du cousin, placés vers le tiers postérieur de la cavité abdominale, sont deux glandes oblongues, cylindroïdes, blanches. Les conduits déférents, plus longs qu'eux, capillaires et presque droits, se renflent en arrière en une poche oblongue, qui lient lieu d'épididyme. Ces deux poches sont si contiguës, qu'un œil peu exercé pourrait croire qu'elles confluent ensemble. 
Les vésicules séminales, confinées au bout de l'abdomen et difficiles à meltre en évidence, se présentent sous la forme de deux grosses utricules ovoïdes ou ventrues, confluentes en arrière, ou a lieu l'insertion des conduits déférents. Le canal éjaculateur est forl court et étroit.

L'armure copulatrice du $G$. annulatus a un forceps, toujours visible au bout de l'abdomen, dont les branches conoïdes, velues en dehors et terminées par un crochet articulé, corné, glahre. presque de leur longueur, sont susceptibles d'un grand écartement et ressemblent aux mandibules des araignées, comme l'avai aussi observé de Géer. A la base inférieure du forceps est une volselle, invisible quand on envisage le bout de l'abdomen par sil face supérieure, et composée de deux petits crochets cornés, noirâtres, courbés en hameçon.

\section{ARTICLE II.}

APPAREIL GÉNITAL FEMELLE.

Les ovaires, dans un état de fécondation avancée, constituent deux grappes oblongues ou allongées, finissant par occuper toute la capacité abdominale, garnies dans leur périphérie de gaínes ovigères, uniloculaires, courtes, oblongues ou subglobuleuses, suivant l'éporque de la gestation, blanchàtres, extrèmement nombreuses (plusicurs centaines), tantôt pressées entre elles sans ordre, tantôt paraissant affecter une disposition par séries longi. tudinales. Ces gaines, pour ainsi dire sessiles, sont dépourvues de ligament propre. Le calice de l'ovaire, par le fait même de l'insertion périphériale des gaines oviggeres, est central. Le col est court, ainsi que l'oviducte, qui est cependant plus long que lui. Les a'ufs à terme sont blancs, ovalaires, ou parfois en courte massue.

La glande sébifique se compose de trois orbicelles à large centre noir, it col efférent, capillaire, flexueux. Contre la règle générale, 
le réservoir séminal est assez gros, ovalaire, subdiaphane, un peu atténué vers son insertion et unique. Nous trouvons dans la glande sébifique l'organe sécréteur de cette matière gluante dont parle Réaumur, ef qui, au moment de la ponte, sert à coller les uns contre les autres les oufs si élégamment disposés dans une attitude verticale, pour former un berceau flottant.

L'oviscaple est excessivement court, et l'on ne peut en bien constater l'existence qu'en examinant à une forte loupe le bout de l'ablomin, soumis à une compression expulsive graduec. En procédant ainsi, on met en évidence uné pièce centrale, cornée, lancéolée, velue, composée de deux lames contiguës, dont lieanmur ne parle pas. On voil aussi deux tentecules velecaires biarticulés, lirms, dont larticle terminal, plus grand et en forme de cueilleron ovale, vhu en dehors, eal bien propre, par lcur action combinée, à saisir doucement les œufs pour les colloquer d'une manière si régulière.

FIIILLE DES TIPLLARES.

Celte populeuse famille, qui, il y a un demi-siècle, ne formait que le seul genre Tijulu, se troure arjourdhui, par laccroisse-

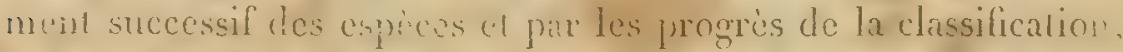
divisie en cint grandes tribus et en plus de soixante genres.

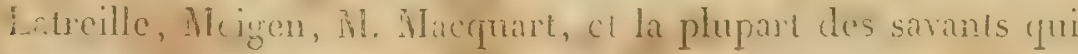

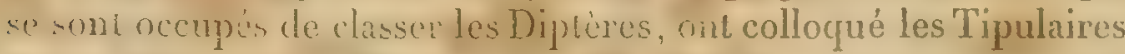
"1 ies Culicides a la tive de cet orlede dinsectes, quoiqu'ils n'aient tomble leurs caractives gue sur línde de la stmeture extéricure. Fa leur assignant, d'un commun accord, ce poste avancé, ils ont "onsacró pour ces familles une prééminence que l'anatomic confime, et dont le sysicme nerveux nous a déjà fourni une preuve hion ecmarguable.

Les Tipulaires qui ont servi à mes dissections sont: 
1. Clenophora pectinicornis. MElG.

2. bimaculata. MEIG.

3. Xjphura atrata. Brut.

4. Tipula oleracea. F.

5. - lunata. L.

6. Pachyrhina crocala. MAce.

7. maculasa. MAce.

8. Anisotoma nigra. LATR.

9. Macrocera hybrida. MeiG.

10. Mycetophila amabilis. DuF.
11. Mycetophila hilaris. Duf.

12. Ceroplatus dispar. Dur.

13. tipuloiles. Bosc.

14. Sciophila strialu. MEIG.

15. Sciara ingenua. Dup.

16. Psychoda ocellaris. LATR.

17. trifasciata. LATn.

18. Rhyphas fenestralis. MEIG.

19. Bibio marci. L.

20. Mycelobia pallipes. MEIG.

J'ai pris pour type de mes descriptions anatomiques la Tipula oleracea, commune dans toutes les contrées, et déjà illustrée par Réaumur, qui nous a fait connaître en détail et ses habitudes et ses métamorphoses (Mćm.t. V, pl.'2 et 3).

1. M. Macquart, dans son excellente Histoire des Diptères, ne donne que treize articles aux antennes du genre Tipula. Il en existe qualorze dans l'oleracca, ainsi que dans beaucoup d'autres espèces: ces articles, mal étudiés jusqu'à ce jour, sont veloutés au microscope, ce qui les rend très-aptes à la fonction tactile; ils sont renflés et comme bulbeux en arrière, et c'est sur ce bulbe et non dans l'articulation que s'implantent les quatre soies verticillées qu'on y voit; le dernier, ou l'article apical, bien plus court que les précédents, est turbiné.

2. Le dernier article des palpes a aussi dans cette espèce et autres une organisation, une structure, qui méritent d'arrêter notre altention. Cet article, plus long à lui seul que les trois autres pris ensemble, a une flexibilité spéciale, déjà signalée par M. Macquart, qui peut se rendre sensible dans l'animal vivant, soit en la mettant en jeu par le toucher, soit en constatant pendant lirritation de l'insecte les diverses contractions partielles qui lui donnent souvent de la difformité. Soumis ả un fort grossissement microscopique, cet organe offre de fines raies transversales plus ou moins flexueuses, couvertes d'un duvet velouté ou en brosse, et faisant l'office de demi-articulations. Cette sou- 


\section{RECHERCHES ANATOMIQUES ET PHYSIOLOGIQUES}

plesse le fait s'accommoder d'une manière très-immédiate aux surfaces des corps pour pratiquer le palper; elle lui permet de se ployer sous la tête, soit pour abriter la bouche, soit pour y introduire les aliments, soit enfin pour exercer avec le secours des poils qui le hérissent une action préhensive. J'ai déjà fait connaitre des structures de cette espèce dans les palpes des phryganes ${ }^{1}$ et dans les appendices caudales d'autres insectes.

\section{CHAPITRE PREMIER.}

ApPAREIL, DIGESTIF.

On sie nous dit pas quelle est l'espéce de nourriture des T'ipulaires; mais la forme et la structure de leur bouche indiquent assez que celle-ci p'est propre qu'à lécher, qu'à humer un aliment liquide ou très-pulvérulent, et mes dissections ne m'ont jamais fait découvrir dans leur canal digestif qu'une lirqueur peu abondante, incolore ou avec une teinte verdâtre.

Fidèle à la maxime de suum cuique, je dois dire que Ramdohr ${ }^{2}$ a publié, il y a environ trente ans, la figure et la description du canal alimentaire des Tip. lunata et oleracea. C'est le seul auteur à ma connaissance qui ait porté le scalpel dans les entrailles des Tipulaires, et il s'est exclusivement borné à l'organe digestif.

J'exposerai aussi, à la fin du chapitre, mes recherches surl'anatomie de leurs larves.

Les glandes salivaires ont dans les Tipula et les Bibio la forme d'une utricule ovoïde oblongue avec un col capillaire; elles sont grêles dans les Ctenophora, Pachyrhina, Rhyphus, bien plus longues dans le Macrocera, où elles pénètrent bien avant dans la cavité abdominale, presque capillaires dans le Mycelophila. Les dissections les plus attentives ne m'ont pas permis de constater leur

- Recherch. anat. et phys. sur les Orthopt. Itymón. Nérope. (Mém. de l'Instit. 184 1, p. 352.)

- Abhandl. ub. die Verd. der Ins. p. 179, pl. 20, fig. 1. 
existence dans les Psychoda; la petitesse et l'extrême fragilité de ces insectes les auront vraisemblablement dérobées à ma vue.

Le tube alimentaire est à peu près de la longueur du corps dans les espèces du genre Tipula de Geoffroy; il est un peu plus long dans les Macrocera, Mycetophila, Ceroplatus, Rhyphus. Celui du Sciophila et du Bibio, genre qui semble faire le passage des Némocères aux Brachocères de M. Macquart, a près de deux fois la longueur de l'insecte: dans le premier, c'est le ventricule chylifique, et dans le second les flexuosités de l'intestin qui forment ce surcroît d'étendue comparative. Le réservoir de la panse a une configuration qui varie et suivant lcs espèces et suivant quelques conditions digestives: dans les Tipula il est ou oblong ou avec un éiranglement plus ou moins ridé ou boursouflé; il est grêle et courbé en hameçon dans les Ctenophora et les Sciophila, tandis que dans le Xyphura il est oblong et droit, ainsi que dans les Pachyrhina, Ceroplatus et Sciara; celui des Macrocera et Mycetophila est grêle et droit; il est au contraire large et bilobé dans le Rhyphus, ovoïde dans le Psychodes. Le ventricule chylifique est séparé de l'œsophage par une valvule intérieure analogue au cardia des grands animaux; dans la plupart des Tipulaires, il est simple et arrondi à son origine. Celle-ci, dans le Ceroplatus, a deux bourses ventriculaires conoïdes, et elle est échancrée, presque bilobée, dans le Rhyphus.

Les vaisscaux hépatiques présentent de notables différences suivant quelques genres. Dans les Tipula, Ctenophora, les Pachyrhina, Anisomera, ils sont capillaires plus longs que tout le canal digestif, très-flexueux, d'un jaune salc ou parfois brunâtres, implantés par quatre insertions isolées, et au lieu d'avoir des bouts flottants, ils constituent deux grandes anses reployées comme ceux des carabiques dans les Coléoptères; ils sont moins longs et -avec quatre bouts libres et borgnes dans la plupart des autres genres. Ceux des Psychoda se renflent à leur origine en une sorte de vísicule biliaire ovale ou oblongue, qui rappelle celle que j’ai décrite dans quelques Cimex. Dans plusieurs autopsies de ces frêles Dip- 
tères, j’ai constaté l'existence de cinq vaisseaux hépatiques, comme dans les cousins, presque aussi souvent que celle de quatre, que je regarde comme le nombre normal. Dans le Rhyphus et le Bibio, ils sont plus gros dans une bonne étendue de leur partie moyenne et ils renferment une bile brune ou violacée.

Limtestin présente dès son origine dans les Tipula, Anisomera, Pachyrhina, Rhyphus et Bibio, un renflement plus ou moins prononcé, dont la texture semble plus serrée. Le reclum a quatre boutons charnus oblongs; il y en a cinq ou six dans le Bibio, et ils sont presque nuls dans les Ceroplatus, Rhyphus, Psychoda.

aPPAREIL digestir de QUelQues larves de 'TIPUlaires.

Quoique les larves ne soient qu'un àge, un état de l'insecte ailé, elles présentent néanmoins, tant sous le rapport de leur conformation extérieure que sous celui de leur anatomie viscérale, une si énorme différence, qu'on peut presque les considérer comme deux êtres distincts.

Je prendrai pour types de cette exposition anatomique les larves terricoles de la $T$. lunata et de la Pachyrh, maculosa, qui sont de grande taille.

Les glandes salivaires ont une conformation diflérente de celles du Diptère: diaphanes et d'une texture très-délicate, elles sont tellement engagées, enchevêtrées dans les mailles des tabliers adipeux, qu'elles éludent facilement les yeux peu familiers avec ces sortes d'investigations. Dans le Pachyrhina, ce sont deux longs boyaux filiformes à circonvolutions agglomérées, dont le conduit excréteur a une finesse plus que capillaire; elles sont aussi filiformes dans la $T$. lunata, mais bien moins longures et simplement flexueuses; celles du Xyphura ont en outre des boursouflures granuleuses sur leurs bords. Ces larves terricoles ne se filant pas un cocon pour leur métamorphose en nymphe, leurs glandes salivaires se bornent exclusivement à la sécrétion de la salive pour l'acte digestif; il n'en est pas ainsi dans les larves fongivores, dont 
plusicurs s'enferment dans une coque: cet organe peut remplir à la fois les fonctions de glande salivaire et de glande sérifique. Ainsi, dans la larve du Ceroplaitus, cette dernière est filiforme et plus longue que tout le corps ${ }^{1}$; elle a cette mème longueur dans celle du Mycetoplita, avec une disposition pinnatifide intérieure à sa partie postérieure ${ }^{2}$.

Le tube alimentaire a pour sa longueur la plus parfaite amalogie avec cclui de l'insecte ailé, mais il diffère beaucoup pour sa composition et sa structure: l'oesophage, excessivement court dans la Tipule, se prolonge dans la larve, bien au delà de la tête, saus être capillaire; celui du ver de la lunate aboutit ì un organe qui n'a pas d'analogue dans l'insecte ailé : c'est une poche globuleuse qui a tous les caractères d'un gésier, car il est revêtu intérieurement de colonnes charnues assez serrées, conniventes, soit en avant, soit en arrière pour former deux valvules. La panse n'existe pas; le ventricule chylifique est un tube membraneux, expansible, droit, embrassé à son origine par quatre bourses ventriculaircs allongées, blanchâtres, collées contre les parois de l'organe et dont les deux inférieures sont plus courtes; ces bourses n'existent pas du tout dans l'insecte parfait.

Les vaisseux hépatiques ont leurs quatre bouts flottants, tandis que dans la Tipule ces bouts doivent se souder ensemble. L'intestin, séparé du ventricule par une constricture annulaire, présente à une petite distance de son origine, et toujours au côté droit, un cocum latéral oblong, qui est destiné à disparaitre dans l'insecte ailé. Le rectum est allongé, plus ou moins ridé, mais sans boutons charnus.

Il n'y a dans la Pachyrhina ni gésier, ni bourses ventriculaires; l'œesophage se dilate en arrière en un jabot ovoïde; le ventricule chylilique, renflé à son origine, présente dà quatre tubercules

- Voir mon ménoire sur les Ceroplatus (Annal. des sciences nat. avril 1839).

2 Voir mon mémoire sur les métamorphoses des larves fongivores des Diptères (Ibid 1839 ). 


\section{RECHERGHES ANATOMIQUES ET P'HYSIOLOGIQUES}

charnus quil faut considérer comme les rudiments des bourses de la larve précédente; le reste est comme dans celle-ci.

Dans la larve de la Xyphura, il existe aussi un jabot sans gésier; mais son trait anatomique différentiel est une double rangée de quatre bourses ventriculaires allongées égales entre elles, l'une a l'origine, l'autre presqu'a la terminaison du ventricule. Leș bourses de la première rangée sont dirigées d'avant en arrière et celles de la seconde d'arrière en avant.

Nous allons trouver dans le canal digestif de la larve si singulière du Ceroplalus, dont j’ai publié l'histoire dans le mémoire précité, des traits organiques qui ne se rencontrent ni dans l'insecte ailé, ni dans les larves terricoles; ce canal a près de trois fois la longueur de la larve. L'oesophage se renfle en un jabot allongé, à parois plissées en travers; il s'implante par un col à un gésier ovalaire à parois calleuses et rénitentes. Le ventricule chylifique, allongé et boursouflé, reçoit un peu après son origine deux bourses ventriculaires sous la forme de boyaux grêles, presque aussi longs que le ventricule. Les vaisseaux hépatiques, remarquables par leur grosseur, qui égale celle de l'intestin, et par leur médiocre longueur, s'unissent par paires en deux canaux cholédoques assez longs; l'intestin est filiforme et se reploie sur lui-même; il n'y a ni coccum latéral, ni rectum marqué.

Le tube alimentaire de la larve du Mycetophila est à peine plus long que le corps. L'osophage s'implante brusquement et sans jabot à un gésier ovoide à parois calleuses; le ventricule chylifique a, dès son origine, deux bourses semblables à celles de la larve du Ceroplatus, mais un peu plus grosses et comme festonnées sur les bords; les vaisseaux hépatiques sont flottants par un bout avec quatre insertions isolées. 
CHAPITRE II.

APPAREIL GÉNITAL.

$\triangle$ RTICLE Ier.

APPAREIL GÉNITAL MìL.

La petitesse, la fragilité et les connexions insolites des diverses parties de cet apparcil en rendent la dissection et l'isolement d'une difficulté extrème.

Les testicules sont ovoïdes, subdiaphanes, situés vers le milieu des flancs abdominaux, enchevêtrés et comme perdus dans le lissu adipeux. Le conduit déférent, d'une ténuité capillaire, est six ou sept fois plus long que le testicule, et présente en arrière un renflement ellipsoïdal constant, souvent revêtu d'une tunique adipeuse jaunâtre. Ce renflement est un épididyme analogue aux épididymes vésiculaires que j’ai fait connaitre dans plusieurs hyménoptères. Les vésicules séminales sont confondues, en apparence, en un seul cordon filiforme courbé en une crosse spiroïdale d'un jaune plus ou moins foncé, parfois safrané. Cette couleur est fournie par une tunique adipo-membraneuse. En arrière, ce cordon, qui n'est qu'un fourreau, reçoit les deux conduits déférents qui s'engagent sous sa tunique; en avant, il se termine par trois vaisseaux simples dont la constatation exige une patience éprouvéc. De ces trois vaisseaux, les deux latéraux, égaux entre eux et fort longs, sont la continuation des vésicules séminales, et l'intermédiaire, plus court, doit être considéré comme le canal éjaculateur. Ces vaisseaux latéraux sont très-reployés, parfois même agglomérés d'une manière inextricable et se terminent par un bout flottant; quand on est assez heureux pour dépouiller le cordon de son enveloppe adipo-membraneuse, on se convaine que les deux conduils déférents, peu après leur entrée dans cette cnveloppe, se dilatent un peu pour constituer les vésicules sémi11. 
nales : ces dernières sont étroitement adhérentes dans leur gaîne, comme sil existait entre elles une membrane.

Le canal éjaculateur a encore plus de capillarité que les vésicules séminales, mais il est plus court qu'elles et je ne l'ai jamais trouvé revêtu d'une tunique adipeuse; à l'endroit où ce canal se dérobe au scalpel pour s'enfoncer dans l'appareil copulateur, il y a des pièces insolites dont les attributions sont encore mystérieuses pour moi, et qui rappellent la complication de l'organe génital mâle de l'abeille à micl.

Ce qui frappe d'abord la vue est un filet brun, corné, élaslique, sétiforme, courbé presque en cercle, libre par un bout, implanté par l'autre derrière ou au-dessous d'une vésiculc centrale dont je parlerai bientôt. Ce singulier filet, que sa texture et sa couleur rendent évidenment destiné à sortir du corps pour l'acte copulatif, est le fourrea de la verge. Une large membrane pellucide, que son excessive minceur rend prescue imperceptible, l'unit dans la plus grande partic de sa longueur avec la vésicule centrale; le bout libre du fourreau, observé à la plus forte lentille de mon microscope, est tricuspidé; les pointes latérales sont légèrement arquées au dehors, celle du milieu est une sorte de stylet ou d'aiguillon droit, renflé vers sa base et terminé par une soie.

La vésicule centrale, assez grande, brunâtre, cornéo-membraneuse ou comme scarieuse, approche pour sa forme des deux tiers d'un sphéroïde; elle est fixée au corps par une base callosocharnue oú l'on aperçit, comme enfoncés dans les chairs, six crochets à peine cornés, dont deux plus grands sont les seuls apparents lorsqu'on n'a pas arraché cette base, et les quatre autres plus petits, mais de mêne nature, restent cachés. Ces six crochets m'ont paru avoir une base commune, et il est vraisemblable que leur principale fonction est de donner attache aux muscles.

C'est la troncature du bout de l'abdomen qui est le réceptacle de l'armure copulatrice, et il suffit d'y exercer une compression expulsive pour déterminer l'exsertion et le développement, l'épa- 
nouissement de ses pièces constitutives. Avant moi, Réaumur avait décrit et figuré cette singulière machine copulatrice; mais nous n'attachons pas peu d'importance à confirmer les faits rapportés par ce profond observateur, et a signaler les crreurs ou omissions qui rendent ses figures défectueuses.

Les pièces copulatrices sont, les unes latérales, les autres centrales: les latérales, au nombre de quatre pour chaque côté, sour établies sur une base ou souche commune, de manière à former un faisseau. En procédant du dehors au dedans, ces pièces sont: $1^{\circ}$ un forceps formé de deux grands crochets cornés, glabres, de couleur ambrée, plus foncée -vers leur extrémité, arcués, en alêne pointue, tellement dirigés que, dans le repos de l'appareil, leur pointe regarde en haut; $2^{\circ}$ deux sortes de raquettes d'un ambré pâle, glabres, dilatées et comme échancrées; $3^{\circ}$ une volselle de deux lames cornées, en sabre courbe, à tranchant arec quelques cils microscopiques près de sa pointe, à dos garni d'une série de soies roides; $4^{\circ}$ deux slylets noirs en lame subaiguë, roide, naissant de la base interne du sabre, non signalée par Réaumur. On voit aussi, près de l'échancrure du bout de l'abdomen, un pinceau de soies rousses et roides inséré sur un talon charnu.

Les pièces centrales se bornent à une tige subcornée trifide, avec les tiges latérales plus courtes terminées par un mamelon, séparées par un sinus marqué de l'intermédiaire dont le bout est conoïde. Par une compression expulsive, j’ai fait sortir de celui-ci un long brin blanchâtre, qui est suivant moi la verge, dont le fourreau était resté à l'intérieur. A une forte lentille microscopique, la verge se termine, d'un côté, par une pointe un peu arquée en dehors, de l'autre par une légère éminence : ce serait là une sorte de gland. La consistance de la verge est élastique; il y a un canal intérieur où j’ai aperçu des gouttelettes. Cet organe n'avait pas échappé à Réaumur, mais il n'en avait saisi ni la structure ni les attributions, et il le prenait pour un filet de sperme.

Je n'avais pas surpris à l'œuvre le filet corné, que sa position, sa texture et ses connexions me déterminèrent à désigner sous 


\section{0 \\ RECHERCHES ANATOMIQUES E'T PHYSIOLOGIQUES}

le nom de fourreau de la verge, mais l'observation suivante vient lever tous les doutes.

Dans le mois de mai, jai fréquemment trouvé dans mon jardin les sexes de la Pachyr. maculosa accouplés et s'envolant de concert. Si on les saisit avec précaution dans ce moment, et qu'on les détache l'un de l'autre, on voit que le pénis sort de la vulve sous la forme d'un filet brun élastique, plus fin que le plus fin cheveu d'enfant, et que sa longueur égale presque celle de l'insecte. Ce filet, une fois dégagé du corps de la femelle, s'enroule, et si vous ne blessez pas le mâle, il rentre peu à peu dans le corps et disparaît entièrement. Disséquez ce mème mâle et vous retrouvez dans la cavité alsdominale ce filet ployé sur lui-même, a peu près comme une de ces longues cravaches de cocher, dont on ramène le bout flexible vers le manche. La tige, qui correspond à ce dernier, se dirige d'arrière en avant, et le filet revient d'avant en arrière. Une membrane hyaline occupe l'intervalle entre la tige et le filet, comme dans la $T$. oleracea. La pointe de celui-ci offre au microscope une ouverture latérale dépassée par un prolongement pointu qui fait l'oflice de prépuce : c'est par cette ouverture que sort la véritable verge.

L'appareil génital de la $P$. crocata est organisé sur le mème plan que celui de l'oleracea, mais moins compliqué. Les testicules sont ovales ou ovoïdes; les conduits déférents, fort longs et grêles, n'offrent pas dans leur trajet le renflement épididymique, mais ils s'engagent pareillement dans un fourreau jaune qui ici fait deux ou trois circonvolutions; les vésicules séminales sont les mêmes.

Celui du sciophila est infiniment plus simple, ses testicules ovales-conoïdes, très-pointus, ont un conduit déférent grêle plus court qu'eux. Il y a deux paires de vésicules séminales, les latérales, allongées et courbées en crosse; les internédiaires, subglobuleuses, pédice!lées; le canal éjaculateur est long, filiforme. Dans l'accouplement, les abdomens sont unis bout à bout, et la femelle remorque te mâle.

Les testicules du Macrocera sont ovoïdes, le conduit déférent 
est capillaire et deux fois plus long qu'eux; il n'y a qu'une paire de vésicules séminales filiformes.

Malgré l'extrême petitesse des Psychoda, j’ai pu constater cet organe de la génération dans l'Ocellaris. Il est d'une grande simplicité; les testicules ovoïles, assez gros, presque sessiles, se terminent par une espéce de bouton globuleux ou par une pointe, suivant le degré de leur turgescence séminale; il n'existe pas de conduit déférent. Il y a deux paires de vésicules séminales, l'une centrale, plus grande, cylindroïde, plus longue que le testicule qu'elle reçoit à sa partie postéricure, et externe; l'autre, comme rudimentaire, oblongue, insérée au-dessous de l'insertion du testicule. Le canal éjaculateur est fort court. L'armure copulatrice se compose de deux paires de crochets, l'une plus inférieure en hameçons cornés, noirs, à concavité de l'arc, supérieure, très-garnie d'un duvet gris; l'autre, supérieure et latérale, de deux articles, le basilaire, court, gros et velu, le terminal en stylet droit, glabre, pointu, se fléchissant sur l'autre. Au centre des crochets, est une lame lancéolée plate, qui appartient sans doute au fourreau de la verge.

Les testicules du Rhyphus sont assez gros, conico-ovoïdes, d'un brun chocolat, atténués en arrière, rapprochés. Le conduit déférent est du même brun, grêle, mais renflé en arrière.

\section{ARTICLE II.}

APPAREIL GÉNITAL FEMELLE.

Les ovaires de la $T$. oleracea occupent, dans un état avancé de gestation, une grande partie de la cavité abdominale et frappent à l'instant la vue par leur couleur noire, comme charbonnée, oủ la loupe attentive aperçoit des granulations blanches formées par la saillie des gaines ovigères. Chacun d'eux consiste en un sac ovale-conoïde, dont les parois molles et expansibles, entourées et pénétrées d'un riche lacis d'arbuscules trachéens, recèlent une quantité innombrable de gaînes ovigères oblongues, uniloculaires, tellement disposées dans toute la périphérie, que leur orifice correspond à une cavité centrale, qui est le calice. II se ter- 


\section{RECHERCIIES ANATOMIQUES ET PIISIOLOGIQUES}

mine en avant par un ligament propre qui s'unit à son congénére pour la formation du ligament suspenseur commun, fixé dans l'intérieur du thorax. Les gaînes sont enfoncées dans les parois de l'ovaire jusqu'à leur bout libre ou ovulaire, qui est seul sursaillant. Lorsque les calices renferment tous les oufs des gaînes, celles-ci, vides et flétries, sont tellement collées contre là face interne du calice ou tellement ratatinées, qu'il est impossible d'en constater l'existence malgré la minceur, la diaphanéité des parois. Mais dans la condition contraire, si on ouve l'ovaire et qu'on en renverse les parois, on voit la face interne de celles-ci hérissée par les gaînes, qui y sont comme pendantes.

Dans d'autres circonstances, peut-être dans un cas pathologique, une espèce d'hypertrophie, les ovulaires ou les bouts sursaillants des gaînes deviennent fort gros, globuleux, et on les dirait remplis, distendus par une graisse compacte, une matière sébacée. Cet état insidicux en imposerait d'autant plus facilement pour des nufs blancs, qu'il ne s'observe guère qu’après la ponte, lorsqu'il n'y a plus d'oufs noirs dans le calice, lorsque toute fonction utérine a cessé. Si licet parvis componere magna, l'état de ces ovulaires est comparable aux engorgements des ovaires qui surviennent chez la femme à l'époque critique.

Les anfs, au nombre de plusieurs centaines, sont oblongs, subcylindroïdes, remarquables surtout par leur couleur trés-noire et luisante. Contre l'assertion de Réaumur, je ne les ai jamais rencontrés courbés en croissant. Ils ne deviennent tels qu'en se déformant par la dessiccation.

L'oviducte est court, mais assez large. La glande sébifıque consiste en trois vésicules ovoïles d'un brun foncé, plus ou moins masqué par une enveloppe adipeuse blanche. Ces vésicules ne sont pas, comme les orbicelles du cousin, noires seulement au centre : chacune d'elles a un col cférent capillaire, reployé, élastique, blanc, excepté vers son origine, où il a une teinte rembrunie. Iscs trois cols aboutissent a un seul conduit excrétcur aussi fin qu'eux et un peu moins long. Les réservoirs séminaux 
sont deux boyaux en massue courbée en crosse, d'un blanc opaloïde, terminée brusquement en arrière en un col court et fin, presque imperceptible. Ces deux cols contigus, mais non confondus, s'insèrent à une espèce de bulbe olivaire ou oblong, implanté sur l'oviducte au même point que le conduit excréteur de la glande sébifique. Lorsqu'on saisit avec une fine pince le bulbe, et qu'on l'arrache avec précaution, on entraine toujours le conduit excréteur. Ce fait semblerait indiquer une connexion anatomique de la glande sébifique et du réservoir séminal, et, par conséquent, une communauté d'attributions physiologiques; mais abstenons-nous encore, jusqqu'à nouvelle confirmation d'une semblable dépendance.

En voyant la couleur très-noire des œufs de notre Tipule, j'avais d'abord pensé qu'une partie de l'appareil en question pouvait bien ètre destinée à la produire; mais en voyant que ces œufs sont noirs dans le calice mème et avant d'aborder l'oviducte, j’ai dû renoncer à cette idée assez séduisante.

Il me reste, pour terminer l'anatomie de la $T$. oleracea, à décrire la structure singulière de l'oviscapte.

Celui-ci, ou l'instrument propre à introdaire les œufs dans un milieu résistant, est placé au-dessous des pièces dorsales du bout de l'abdomen contre lesquelles il est appliqué dans l'inaction, mais dont il peut s'éloigner dans l'exercice de ses fonctions. Il représente en petit celui des sauterelles. Il est essentiellement constitué par deux lames allongées, effilées, cornées, brunes, glabres, creusées en gouttière superficielle d leur face interne; leur base dilatée se fixe par des muscles nombreux et puissants au bout de l'abdomen. Réaumur (l.c. vol. V, p. 19) nous a fait connaître la manière dont les Tipules procèdent à la ponte de leurs oufs; j'ai été témoin de cette opération dans la Xyphura: elle enfonce presque tout l'abdomen dans le terreau et renouvelle fréquemment sur divers points cette insertion, en sorte qu'il est présumable qu'elle ne pond qu'un œuf à la fois.

Les ovaires ont la même forme générale, la même structure et 
des œufs à terme pareillement noirs daus les Pachyrhina, Clenophora et Iyphura; dans ces deux derniers genres, l'organe sécréteur de la glande sébifique se compose de trois orbicelles à centre brun. Les réscrvoirs séminaux ont dans la Xyphura un col plus long qui sinsère directement, et non au moyen d'un bułbe commun, à l'oviducte.

Les ovaires du Ceroplatus (dispar) sont allongés avec des gaînes ovigères uniloculaires, innombrables; le calice est central, le col fort court, l'oviducte aussi et bulbeux à son origine, les ocufs sont sphériques et blancs, au moins dans les ovaires. Après une macération peu prolongéc dans l'eau, ils ont pris une couleur noire, comme ceux des Tipulaires terricoles, ce qui me fait présumer qu'ils deviennent peut-être noirs après avoir été pondus. La glande sébifique difrére beaucoup des précédentes, les vésicules sécrétrices sont ovalaires, blanches, à parois épaisses et au nombre de deux seulement; leur col, plus court qu'elles, est brusquenent capillaire, et chacun d'eux s'insère isolément; les réservoirs séminaux soni deux boyaux fusiformes presque aussi longs que les ovaires, et pellucides. L'oviscaple, qui repose sur la concavité du dernier segment ventral de l'alodomen, est formé de deux lames subtriangulaires aiguës, susceptibles de déduction, hérissées de quelques poils et articulées à l'échancrure d'une pièce basilaire; les tentacules vulvaires sont arrondis, subcoriacés, brièvement ciliés, d'une seule pièce. La brièveté de l'oviscapte me porte à croire que le céroplate doit déposer ses oufs, non dans l'intérieur de la terre, mais simplement dans les anfractuosités des bolets parasites qu'habite sa larve.

Les ovaires du Macrocera ont la forme de ceux du céroplate, mais les gaines ovigères sont quadriloculaires, disposées sur deux rangées unilatérales, assez lîches, et les oeuls, ovales-oblongs, sont blancs, même à terme; les vésicules sécrétrices de la glande sébifique ne sont qu'au nombre de deux, ovales, noires presque sessiles; il y a deux réservoirs séminaux oblongs, cylindroïdes, diaphanes. 
Les gaines ovigères dans le Mycetophila amabilis n'occupen que la paroi supérieure de l'ovaire, en sorte que le calice est inférieur; les vésicules de la glande sébifique sont doubles, sphéroïdales, diaphanes, longuement pédicellées, et il n'y a pour réservoir qu'un boyau allongé.

Les gaînes ovigères du sciara sont disposées comme dans le Mrcelophila, elles sont uniloculaires et sur quatre rangées longitudinales. La glande sébifique semble se composer de deux paires de bourses ovales-oblongues, diaphanes, atténuées en arrière en un col capillaire de leur longueur; l'antérieure, du double plus pelite, est l'organe sécréteur; la postérièure serait le réservoir séminal.

Les ovaires du Psychoda (trifasciata) sont grands, vu la taille de l'insecte, ovales, obtus, déprimés, garnis dans toute leur périphérie de gaînes ovigères uniloculaires, courtes et grosses, pressées en séries longitudinales; les œufs sont globuleux el blancs.

Les gaînes ovigères ont la même disposition dans le Rhyphus et le Bibio. La glande sébifique de ce dernier a trois orbicelles à centre noir longuement pédiccllés.

\section{FAMILLE DES TABANIENS.}

Les taons, par leurs habitudes sanguinaires, se sont fait remarquer aux époques les plus reculées, et, au rapport de Latreille (Cours d'entom.), ce sont les Myops et les Astros d'Aristote, les Tabani et Asili de Pline. Ils offrent un trait singulier, sur la trace duquel nous avaient déjà mis les cousins. Non-seulement le mâle et la femelle ne partagent pas le même genre de vie, puisque ce dernier sexe est le seul qui blesse les animaux pour s'abreuver de leur sang, remarque déjà faite par de Géer (Mém. t. VI, p. 214 ), mais leur suçoir se compose d'un nombre différent de pièces, de sept dans la femelle et de cinq seulement dans le mâle; de plus, les palpes de ce dernier sont plus courts et redres- 
226 RECIERGHES ANATOMIQUES ET PHYSIOLOGIQUES

sés, tandis que ceux de la femelle sont longs et coucliés sur la trompe.

La position de la famille des Tabaniens à la suite des 'Tipulaires, malgré leur énorme dissemblance à l'état parfait, est aussi justifiéc par la forme et le genre de vie de leurs larves; celles-ci, dans le Tabanus, sont hémicéphalées, cylindriques, à une seule paire de stigmates logée dans une caverne du bout postérieur du corps, et vivent dans la terre.

Les espèces qui ont servi à mes vivisections sont:

1. Pangonia marginala. F.

2. Tabanus bovinus. L.

3. - morio. LATR.

4. fulvus. Merg.
5. Tubanus tropicus. L.

0. bromius. L.

7. Hematopota pluvialis. MEsa.

8. Chrysops cecutiens. ME⿺s.

Après les savantes recherches de M. Macquart sur les langonies (Annal. de la soc. ent. t. VI, p. 429 , et Dipt. exot. I. I, p. 93) tant indigènes qu'exoliques, ce n'est pas sans hésitation rue j’aborde ce.sujet, lorsque surtout je n'ai à parler que d'une seule espèce déjà connue. On ne nous a encore rien appris sur leurs habitudes. J'ai étudié la $P$. marginata, soit en Espagne, soit dans le midi de la France; jai remarqué que cet insecte se plaisait sur les collines voisines des cours d'eau, ce qui me porte à croire que sa larve doit se trouver, comme celle des Tabanus, dans les terres humides du littoral. Dans les jours chauds de juin et de juillet, je l'ai souvent aperçu bourdomnant dans les airs, non avec le timbre aigu du bombyle, mais plutôt avec celui du taon; tantôt il demeure suspendu et presque immobile, comme le syrphe et le bombyle, tantôt il change brusquement de place, ou pour éviter un danger ou pour poursuivre sa femelle, tantôt. enfin, on le voit s'abattre et disparaiture dans les buissons; d'autres lois, il se pose sur les fleurs, et sa trompe s'enfonce dans les corolles tubuleuses pour en sucer le nectar. J'ai trouvé un liquide sanguin dans le canal digestif d'une femelle.

Je crois rendre service a la science en exposant avec quelque 
détail la structure de la trompe de la pangonie et en l'accompagnant de figures qui m’ont paru mieux répondre aux exigences actuclles que celles de Meigen ${ }^{1}$; le rapprochement de cette structure de celle du taon, en mème temps qu’il confirme cette dernière, justifie de la contiguilté de ces deux genres dans la série entomologique.

La trompe de la Pangonia, comme celle da Tabanas, se compose de sept pièces dans la femelle, et de cinc seulement dans le màle, savoir : un fourrean et un suçoir qui est l'assemblage de six ou quatre lames cornées.

Ce fourreau est noir, coriacé ; souple, et s'ouvre par une fente dorsale longitudinale destinée à favoriser le jeu du suçoir; mais celte fente ne se continue pas jusqu'au bout du fourreau. Ce bout, qui a lat forme d'un gland ovalaire, a une organisation spéciale; une ligne médiane, élargie en arrière, le partage en deux moitiés égrales formant une louche à deux lèvres. Une loupe trèsattentive constate à celles-ci, sur l'insecte vivant, de légers mouvements vermiculaires, et le microscope y reconnait des plissures transversales: c'est évidemment un organe destiné à palper et à presser. Il est séparé du corps du fourreau par une demi-articulation cqui lui permet des mouvements propres de totalité. Le corps du fourreau, soumis au plus fort grossissement, offre une texture bien propre à expliquer sa souplesse; ce sont des séries transversales fort serrées de points opacyues tégumentaires sur un fond submembraneux. Quand on comprime expulsivement ce fourreau par sa base, Jorsqu'il renferme les lames du suçoir, on peut en provoquer à volonté, par la fente dorsale, l'issue et le développement. C'est dans ce dernier état que j’ai figuré le suçoir pour l'intelligence de sa composition.

Les six lames du suçoir sont toutes de même longueur et linéaires. D'aprés leurs attributions, on peut les diviser en deux valves, deux lancetles, une langue et un hypoglosse.

Les valves sont les deux lames externes du faisceau, qui tendent Dipl. curop. Lab. 13, fig. 3,4 . 


\section{RECHERCHES ANATOMIQUES ET PHYSIOLOGIQUES}

a presser entre elles, dans le repos, les autres pièces du suçoir; elles sont fort acérées, d'un brun foncé et marquées d'une nervure médiane. Je me suis bien assuré que chacune d'elles s'insère et adhère à la base interne du palpe, de manière que le mouvement de ce dernier peut se combiner avec celui de la valve. Iatreille avait aussi remarqué cette insertion.

Les lancettes, situées au côté interne des valves, sont plus minces que celles-ci et d'une couleur plus claire, mais dépourvues de nervure médiane et à double tranchant; elles accompagnent la langue, qui est logée entre elles. Ces lancettes sont les lames qui manquent dans le suçoir des mâles, et elles jouent un rôle principal dans l'aclion de piquer ou d'inciser le derme des animaux.

La langue, plus fine et plus pâle que les autres lames, a une nervure médiane à peine sensible, qui s'efface avant sa pointe.

L'hypoglosse, situé à la partic inférieure et centrale du faisceau et d'un brun foncé, est creusé en dessus en gouttière, ou canaliculé, et terminé par une sorte de capuchon. Les bords de la goutlière ont une nervure, et celle-ci conflue avec sa congénére pour border l'entrée du capuchon; le fond ou la cavité de la gouttière a quatre nervures, dont les deux intermédiaires, un peu plus saillantes et conniventes à leur extrémité, semblent plus particulièrement destinées à servir de coulisse ou de sonde cannelée à la langue, tandis que les latérales, facilitant le jeu des lancettes, se continuent avec la marginale du capuchon.

Le mécanisme de la succion est des plus simples. Le fourreau, qui répond à la coulisse charnue de Réaumur, en même temps qu'il sert de régulateur et de point d'appui aux lames du sucoir, soppose aussi à l'extravasation du liquide alimentaire. Ce sont évidemment les lancettes qui dardent la peau, qui l'incisent plus ou moins profondément; aussi manquent-elles dans le mâle, et ce trait négatif eût seul suffi pour nous faire penser que ce sexe ne pouvait pas être sanguinaire. Les lancettes sont aidées dans leur fonction par la pression des valves, qui, elles-mêmes, sont 
soumises à l'action des palpes; la langue s'insinue alors comme un instrument piquant dans la blessure, elle aspire le sang, qui glisse dans les rainures et entre les lames pour gagner le pharinx. Observez bien que la texture érectile du bout bilabié du fourreau lui permet de faire l'oflice d'une ventouse. Quelle admirable organisation!

\section{CHAPITRE PHEMIER.}

APPAREIL DIGESTIF.

Je ne connais, sur la splanchnologie des Tabaniens, que la description du canal alimentaire du T. tropicus, par Ramdohr ${ }^{1}$, et celle que j'en ai donnée, il y a plusieurs années, dans le T. bovinus"2.

L'étude comparative des contenta du canal digestif des deux sexes de plusieurs espèces de 'Tabaniens m'a mis à mêne, d'une part, de m'assurer que jamais les mâles n'avaient sucé du sang, et de l'autre, de confirmer le fait énoncé par Réaumur (l.c. t. IV, p. 230), que des taons femelles, conservés dans des poudriers, s'accommodaient d'un aliment sucré. J'ai effectivement rencontré. dans mes vivisections, des individus de ce dernier sexe, où l'estomac renfermait un liquide qui n'était pas du sang, quoique je les eusse pris suçant des animaux. J'en avais conclu que les taons pouvaient bien parfois ne sucer que les liquides incolores de la peau ou la lymphe, comme je l'ai déjà dit en parlant des cousins, et j’expliquais l'effet de leur piqûre par celui d'un vésicant ou d'une brûlure qui déterminent sur notre peau des ampoules de sérosité ou des phlictènes. De ces faits et d'autres observations, j’ai tiré cette double consćquence, $I^{\circ}$ que les femelles des Tabaniens peuvent se nourrir, tantôt de sang, tantôt d'un aliment liquide incolore; $2^{\circ}$ que les mâles ne sucent jamais le sang des animaux, que leur bouche est, anatomiquement parlant, inhabile

3 Ouvr. cit. p. 181, tab. 21, fig. 1, 2.

s Journ. de phys. Mlai 1820 . 
à percer, à inciser des téguments, et qu'ils s'alimentent souvent du pollen des fleurs. Cette induction est aussi applicable aux Pangonia, même aux espèces à très-longue trompe, puisçue, je le répète à dessein, jai constaté du sang dans l'estomac de la iemelle de la $P$. marginata.

Les glandes salivaires des Tabaniens sont grêles, fililormes, reployées dans le $T$. bovinus cn une agglomération assez lâche, moins Inngues et presque droites dans le $T$. tropicus et le $T$. fulvus, courbées en hameçon dans le $T$. bromius, beaucoup plus comrles et atteignant à peine la base de l'abdomen dans l'Hemalopola, presque aussi courtes et terminées par un léger renflement oblong daus le C'hry' sops. Celles de la Pangonia présentent cela de particulier, que le bovau sécréteur forme dans l'abdomen une agorlomération comme dans le $T$. bovinus, tandis que le col, bien plus long que dans ce dernier, traverse tout le corselet avec un diametre capillaire.

Le tube alimentaire a deux fois environ la longueur de l'insecte, comme dans les derniers genres des Tipulaires. L'arsophage n'est un peu renflé ou turbiné que dans la P'angonia; le réservoir de la panse est bilobé; le ventricule chylifique ciébute par deux lourses conoïdes, terminées par un fin ligament, granuleuses comme la portion thoracique de l'organe. Les granulations de celui-ci sont arrondies, souvent disposées en séries longitudinales, et s'elfacent en arrière dans le $T$. bovinus, tandis qu'elles se continuent jusqu'à l'abdomen dans le T. tropicus. En entrant dans la cavitú abdominale, le ventricule offre un renflement sphéroüdal, conoïde ou allongé, suivant quelques circonstances digestives. On observe à ce renflement quelques papilles subglobuleuses isolécs et clair-semées qui n'existent ni dans l'llematopota ni dans la Pangonia.

Les vaisseaux hépatiques s'implantent par quatre insertions isolées et se terminent par autant de bouts flottants; je nc les ai trouvés variqueux que dans l'Hcematopola et le Chrysops. Ordinairement blancs dans le $T$. bovinus, ils passent aussi au jaune vif, suivant le degré d'élaboration de la bile; ceux de l'Hematopota sont violacés. 
L'intestin commence par une dilatation qui égale celle du ventricule, puis il devient filiforme, fait une circonvolution et se termine par un rectum ovalo-conoïde, relevé par quatre ou cinq boutons charnus orbiculaires et subombiliqués en dehors, mais conoïdes dans l'épaisseur de l'organe.

\section{CHAPITRE II.}

APPAREIL GÉNITAL.

ARTICLE I"e?

APPAREIL GENITAT. MALE.

Les testicules du $T$. ater sont conico-ovoïdes ou pyriformes, suivant leur degré de turgescence; les conduits déférents sont plus longs qu'eux, capillaires et flexueux; les vésicules séminales, plus ou moins sphéroïdales et contiguës, reçoivent les conduits précédents à leur bout antérieur et s’atténuent en arrière en un col fort court; le canal éjaculateur, moins long que les conduits déférents, est grêle et à peu près droit.

Tous ces organes ont la mème configuration, la même structure dans le $T$. fulvus. Le testicule de la Pangonia est sphéroïdal, mais le conduit déférent est celui des T. tabanus. Quant aux vésicules séminales, elles mont paru faire défaut. Les conduits déférents s'insèrent an-dessous de la dilatation en crosse du canal éjaculateur; cette dilatation, qui remplace les deux vésicules séminales du T'. tabanus serait-elle divisée en deux? Je ne le pense pas, car cette dissection est très-facile.

L'armure copulatrice du Tater est, dans le repos, presque entièrement cachée sous le bout de l'abdomen. Le forceps, grand, robuste et corné, a ses branches cylindrico-conoïdes, brunes, glabres, terminées par un crochet grêle, presque droit, fléchi et appliqué contre elles quand il ne fonctionne pas, et dont l'extrémité a deux pointes divergentes : l'une aiguë, l'autre obtuse. 


\section{REGHERGHES ANATOMYUES ET PHYSIOLOGIQUES}

Entre ces branches, on voit saillir, et souvent les dépasser, une paire de pièces contiguës biarticulées, coriacées, noirâtres. L'article basilaire est subquadrilatère et glabre, tandis que le terminal est en forme de raquette ovale-arrondie et velue; quand on arrache cette pièce sur l'animal frais, on trouve qu'à l'article basilaire s'implante une sorte de tige cornée grêle, qui se porte obliquement vers la racine du forceps. Le fourreau de la verge est lancéolé, coriacéo-membraneux, avec la pointe un peu échancrée sur un côté.

L'armure copulatrice de la Pangonia a un forceps en fer à cheval, corné, d'un roux ambré, à bouts obliquement tronqués, armés chacun de deux crochets qui font la pince, soit entre eux, soit avec ceux du côté opposé, comme les chéliféres des Crustacés. Je n'ai aperçu entre ces branches rien qui soit analogue aux pièces biarticulées du $T$. labanus. Le fourreau de la verge a deux baguettes écartées à leur base et confluentes à leur extrémité.

\section{ARTICLE II.}

APPAREIL GÉNITAL FEMELLE.

Les ovaires des Tabaniens ( $T$. bovinus) ont la même organisation générale que ceux des Tipulaires. Ils sont formés par deux grappes oroïdes d'innombrables gaînes ovigères oblongues, subbiloculaires, blanchâtres, très-serrées, garnissant toute leur périphérie et souvent rangées en séries longitudinales; ils sont renfermés chacun dans une enveloppe hyaline et se terminent par un ligament propre, puis par un ligament suspenseur commun fixé, comme à l'ordinaire, dans le thorax. Le calice est central, le col trèscourt, l'oviducle aussi; les œufs à terme sont oblongs ou allongés.

La glande sébifıque a pour organe sécréteur trois orbicelles spatulés, noirs au centre, à conduits efférents capillaires longs, courbés en anse, de manière que les orbicelles regardent en arrière et sont insérés isolément à l'origine de l'oviducte, quoique rap- 
prochés; ils offrent au microscope un vaisseau inclus, mais non strié. Les réservoirs séminaux sont deux boyaux filiformes implantés à côté des conduits efférents; ils renferment une humeur hlanche.

Les T. tabanus n'ont pas d'oviscapte proprement dit. Par une compression cxpulsive sur l'abdomen du T. bovinus, on procure d'abord l'exsertion d'un segment noir et échancré; on voit ensuite le vagin plus on moins renversé, dont l'orifice est flançué de deux tentacules vulvaires biarticulés, coriacés, pâles et duvetés. L'article terminal est ovale, très-obtus. L'absence d'un oviscapte saillant prouve que les taons n'enfoncent pas leurs oufs dans m milieu résistant, mais les déposent simplement dans la terre molle ou dans la boue oú vivent leurs larves.

Les ovaires et leurs annexes sont, dans la Pangonia, fort approchants de ceux du $T$. tabanus; la couleur est d'un vert pistache fort remarquable, et les gaînes ovigères ne sont qu'uniloculaires; quant à la glande sébifique, elle n'en diffère qu'en ce que les orbicelles ont des conduits beaucoup plus courts et droits, tandis que les réservoirs sont plus longs et courbés sur eux-mêmes.

FAMLLE DES STRATIOMYDES.

Son caractère anatomique le plus distinctif est l'existence d'un seul canal cholédoque pour les quatre vaisseaux hépatiques.

J'ai disséqué les espèces suivantes:
1. Subula murginata. Mice.
2. Beris vallata. MEIG.
3. Strat chameleon. F.
4. strigata. F.
5. Ephip, thorecicum. LAtr.
6. Odontomyia tigrina. $\mathrm{T}$.

11.
7. Olontonyia furcatc. Latr.

8. Sargus cuprarius. T.

9. Chrysomyia formosa. MAcQ.

10. politu. Mace.

11. Vappoater. Latr.

12. - pallipennis. MacQ. 


\section{CIIPITRE PREMIER.}

IPPAREIL DIGESTIF,

Nous sommes encore peu fixés sur l'espèce de nourriture que prennent ces Diptères. J'ai souvent trouvé le Str. strigata et les Chrysomyia sur les fleurs onbellifères, occupés à en éplucher les étamines. D'autres genres' semblent vivre des sucs impalpables des feuilles, à la face inférieure desquelles ils ont l'habitude de stationner.

Les glandes salivaires, que je n'ai pas su découvrir dans les Subula ct Beris, où je les suppose, sont en général moins développées que dans la plupart des autres familles, parfois comme rudimentaires et ne dépassant pas le thorax; elles sont filiformes dans l'Ephippium et les Sargus, oblongues, cylindroïdes dans les Stratiomys, les Odontomyia, courtes et ovoïdes dans les Vappo.

Le lube alimentaire a dans le plus grand nombre près de deux fois la longueur de l'insecte, trois fois dans les Sargus et Vappo. La panse a un réservoir ovoïde dans le Beris, bilobé dans les autres. Le venlricule chylifique débute par deux bourses généralement assez courtes, mais plus allongées dans les Stratiomy's et Odontomyia. En entrant dans la cavité abdominale, il se renfle, puis s'atténue de nouveau; il est le plus souvent droit ou à peine Hexueux. Celui dı. Sargus fait une circonvolution sur lui-mème.

Les vaisscaux hépatiques avaient jusqu'ici quatre insertions ventriculaires isolées; mais dans les Stratiomydes ils confluent tous ensemble en un tronc commun, un seul canal cholédoque, trèscourt dans les Subula et Beris, plus long et plus gros dans le S. chameleon et les Odontomyia, d'une longueur moyenne dans les autres. Ils sont proportionnellement moins longs que dans les Tabaniens el les Tipulaires, plus ou moins bousouflés, excepté vers leur insertion, diaphanes ou remplis d'une bile d'un blanc amylacé, comme dans les Stratiomys et Sargus. Il n'est pas rare que deux 
de ws vaisseaux, les antéricurs, soient sensiblement plus gros: lEphippium et le Chrysomyia en fournissent des exemples. Ceux du Suryus présentent cela de particulier, qu'ils sont bien moins longs que dans les autres Stratiomydes, mais tous quatre égaux et variqueux, excepté vers leur insertion. Dans les deux Vappo, ils offrent un trait fort singulier, qu'ois peut regarder comme générique : les deux vaisseaux postérieurs sont extrêmement courts, rudimentaires, tandis que les antérieurs sont longs, plus gros et d'un blanc mat, excepté dans leur tiers terminal, où ils sont brusquement plus lins et diaphanes.

Lintestin se renfle d'abord plus ou moins, et devient ensuite filiforme et flexueux. Le rectum a dans l'Ephippium une série circulaire de six boutons charmus oblongs et jaunâtres, de quatre dans le Sargus. Celui du Vappo, presque pyramidal et brusquement échancré à sa base, offre en dessus une ligne médiane enfoncée et n'a pas de boutons charnus apparents.

\section{CHAPITRE II.}

IPPAREIL GÉNITAL.

\section{ARTICLE I ${ }^{\text {er }}$.}

APPAREIL GÉNITAL, MÂLE.

Les testicules sont ovoïdes dans les Subula, Beris, Sargus, Chrysomyia; allongés, cylindroïdes et courbés en hameçon dans l'Ephippium, l'Odontomyia tigrina; filiformes dans l'Odontomyia furcata; en longue massue, plus ou moins fléchie, dans le S. strigata; gros et pyriformes dans le S. chameleon, sphéroïdes dans le Vappo. Les conduits déférents sont tellement agglomérés dans le Beris que ses testicules paraissent sessiles. Ce n'est que par la macération qu'on parvient à les dérouler et à mettre en évidence leur ténuité capillaire et leur longueur, qui égale deux fois an moins celle du testicule. Ceux de l'Ephippium sont, au contraire, du double plus 


\section{RLCHERCHES ANATOMLUES E'T PIYSIOLOGIQUES}

courls que cet organe, capillaires et insérés à nu sur la vésicule sćminale, à une distance assez grande de l'origine du canal éjaculateur, tandis que, dans les autres Strationydes, cette insertion a licu immédiatement avant cette origine. Ces conduits ont au moins la longueur du testicule dans le Strigata et bien davantage dans le Chamalcon. Ceux du Sargus, tout aussi longs, ont une teinte jaune; ils sont moins longs et un peu bulbeux à leur origine dans le Chrysomyia et le Vappo. Dans les Odontomyia les conduits déférents, aussi longs, aussi capillaires que dans le Beris, présentent une disposition toute particulière, dont je n'ai pas encore conslaté d'autre exemple dans les Diptères: ils sont enroulés ensemble en une agglomération globuleuse et centrale, d'un jaune tomate dans le 0 . tigrina, d'un jaune plus pur dans le $O$. furcala, couleurs qui ne sont, comme dans beaucoup de testicules, qu'extérieures et dépendantes d'une tunique subadipeuse. Cette pelote est un véritable épididyme, qui a celte particularité de constituer une masse commune. Je suis jarvenu à isoler les deux conduits et à mettre en évidence leurs insertions aux vésicules séminales correspondantes. Il ne faut pas confondre cette agglomération des conduits défércnts avec celle des véritables testicules, qui s'observe dans quelques Asiliques, ni avec celle des vésicules séminales, dont les Bombyliers offrent des exemples.

Les vésicules séminales, au nombre de deux, sont en général filiformes, llexucuses ou reployées, de médiocre longueur dans les Beris, Sargus, Chrysomyia; celles des Ephippium, Stratiomys, Odontomyia et Vappo sont très-longues et entrelacées.

Le canal éjaculateur est grêle, fort long et flexueux dans le Sabula, médiocre dans le Beris et les Odontonyia, plus court dans l'Ephippium, assez long et atténué en arrière dans le Strigata, court et bulbeux à son origine dans le Chamcelcon, trés-court dans le Sargus, assez long et capillaire dans le Vappo.

L'armure copulatrice du Beris, considérée par sa région dorsale, met en évidence : $1^{\circ}$ un demi-segnent tégumentaire noirâtre, à peine échancré śur les côtés; $2^{\circ}$ une lame presque carrée, glabre 
d'un blond clair, légèrement échancrée en arrière avec les angles postérieurs obtus; $3^{\circ}$ deux appendices ovalaires planes, noirs, velus, d'un seul article, insérés à ces angles; $4^{\circ}$ une lame sub)triangulaire pâle, à peine villeuse, apparaissant à l'échancrure de la lame carrée; $5^{\circ}$ de chaque côté, à la base de celle-ci et en dessous un fort crochet corné, arqué, pâle, velu, formant les branches du forceps. Vue par sa région ventrale, cette armure présente une large pièce basilaire cornée, aux angles postérieurs de laquelle s'articulent les branches du forceps, et qui est armée au côté inteme d'une dent aiguë. Le fourreau de la verge est formé de deux baguettes linéaires brunes, terminées chacune par une pointe allongée pâle; ce fourreau est bordé par une lame coriacée pâle, qui se continue jusqu'à l'endroit où le canal éjaculateur pénètre dans l'armure.

Celle-ci, dans le Strigata, rappelle par sa composition celle des Hyménoptères: la pièce basilaire est en écusson convexe ef arrondi; les branches du forceps, oblongues, grosses et comme sinueuses, sont presque bifurquées à leur extrémité; la dent la plus interne est aiguë, et l'autre obtuse; le fourrean de la verge, d'un brun paile, est formé de deux hagueltes adossées, termineres par un bouton ovalaire.: De dessous une lame sous-jacente au fourreau, on voit saillir deux soies roides roussâtres et la pointe obtuse des branches du forceps est débordée au côté externe parr une pièce oblongue coriacée, velue en dehors, voilà ce qu'on aperçoit à la région dorsale. A l'opposć, on voit une sorle de volsclle formée de deux branches olstuses brunes, à l'extrémité desquelles sinsère la pièce oblongue que j’ai dit déborder le bout

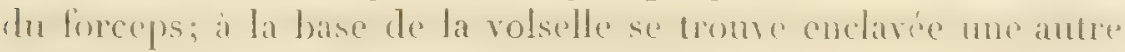
petite volsellc. En dessous, le fourreau de la verge se ternime par un corps ovoïde pointu, ambré, enchâssé en arrière dans une pièce qui se prolonge de chạque còté en un stylet sétiforme.

L'armure copulatrice du Sargus, fort petite et presque quadrilatère, est noirâtre et cornéo-coriacée; le forceps est court, terminé par un crochet velu en dehors, et garni en dedans de 
quatre ou cinq soies roides; entre les branches du forceps, il y a une lame cornée échancrée. Le fourreau de la verge sort de dessous l'échancrure de cette lame; en renversant l'armure on apercoit deux appendices allongés, velus, fixés à la base d'une pièce subtriangulaire.

AR'TICLE II.

APPAIEIL GÉNITAL FEMELLE.

Les ovaires, ovales ou oblongs, sont, à proprement parler, des épis composés de gaînes ovigères sessiles, innombrables, ovales ou globuleuses, uniloculaires dans l'Ephippium, les Odontomyia, le Sargus, biloculaires dans le Beris, à trois ou quatre locules dans le Vappo. Le calice est central, le col bien marqué, ainsi que l'oviducle; les oxufs sont en général oblongs, atténués à un bout, subglobuleux dans les Odontomyia.

La glande sébifique du Beris et de l'Odontomyia (ligrina) a trois orbicelles ovalaires à centre noir, contigus sur une même ligne, avec des conduits efférents capillaires, parfois fléchis tous trois au même point, et plus ou moins renflés avant leur insertion.

Les réservoirs séminaux sont filiformes, blancs; dans le Beris, ils m'ont paru confluents en arrière en un seul tronc fort court; les tentacules vulvaires sont biarticulés, velus.

Gette glande est très-différente dans l'Ephippium; l'organe sécréteur est ici représenté par deux corps de forme et de structure insolites: l'un esi un globe diaphane, d'une consistance comme calleuse, muni d'un conduit eflérent assez court; l'autre est une grande anse épaisse, subcalleuse, ayant un vaisseau inclus blanc, et offrant avant son insertion à la paroi supérieure de l'oviducte, une espèce de bulbe de couleur jaunâtre, et d'une texture particulière. Par le bout opposé, clle dégénère en un vaisseau capillaire dont je n'ai pas bien vu la terminaison, et qui parfois m'a paru s'implanter -au point même d'insertion de l'anse. Les réser- 
voirs séminaux sont deux vaisseaux fort longs, blancs, très-fins. fragiles, entremêlés, ayant trois ou quatre fois la longueur de l'insecte; l'oviscapte se compose de trois tuyaux engaînés, légèrement velus, et se termine par deux tentacules valvaires biarticulés. relus.

La glande sébilique du Sargus va nous présenter un de ces types de transition intéressants à constater : l'organe sécréteur est un paquet de trois longs filets tubuleux, simples, ployés d'avant en arrière et làchement entrelacés. Les réservoirs séminaux sont deux boyaux simples, grêles comme un fil.

Dans le Chrysomyia, ces trois filets sont terminés par une.vésicule oblongue.

La même glande se compose, dans le Vappo palliponnis, de trois vảisseaux sécréteurs. simples, remarquables par une grosse vésicule ovale-globuleuse terminale, diaphane; an centre de celleci on découvre, comme une sorte de caroncule, un tout petit filet tubuleux terminé par un capitule. Les réservoirs sont aussi deux longs vaisseaux simples, d'une ténuité capillaire. L'oviscapte peut s'allonger au point de surpasser en longueur celle de son abdomen; il se compose de trois tuyaux grisâtres à peu prés égaux, et de deux autres plus pâles et d'une consistance molle: cette longueur et cette structure de l'oviscapte nous annoncent d'avance que les Vappo doivent enfoncer leurs œufs très-profondément dans une substance peu résistante : on sait, en effet, que les larves de ces Diptères vivent dans le hois pourri et décomposé.

FAMILLE DES ASILIQUES.

Les Asiliques, tels que les comprenait Latreille, au beau temps de son Genera, constituent une des familles les plus naturelles, les plus légitimes de l'ordre, et ce même auteur a violé ses propres principes en composant son étrange famille des 'Tanystomes avec des groupes si disparates; il suffit de nommer l'Asilus et le 
Bombylius, pour prouver combien ils sont peu faits pour fraterniser.

Ils comptent dans leurs rangs les Diptères les plus grands; les plus robustes, el en même temps les plus intrépides; ils sont chasseurs de profession : on les voit, au plus fort de l'été, se tenir en embuscade sur les chemins, les tertres, les trones d'arbres, se placer en arrêt comme un chien couchant, s'élancer sur leur proie et l'emporter dans les airs, quoiqu'elle ait souvent plus de volume qu'eux; leurs paltes, armées de nombreux piquants, leur servent admirablement pour saisir et retenir les insectes.

Voici les espèces dont j’ai fait l'autopsie:

1. Laphria fulva. Merg.

2. Dasypogon teulonus. F.

3. punclatus. 'T'.
4. Diociria nigritarsis. Nos.

5. Asilus crabroniformis. T.

6. - senex. MeIG.

\section{CHAPITRE PREUIER.}

APPAREIL DIGESTIF.

Les Asiliques sont insectivores, mais ils ne se bornent pas à sucer leur proie, ils la déchirent, ils la broient pour s'en nourrir.

Les glandes salivaires sont aussi peu développées dans les Asiliques que dans les Stratiomydes, et leur longueur atteint à peine le milieu du thorax: ce sont deux boyaux plus ou.moins flexueux, gros et bulbeux à leur origine dans $1^{\prime} 1$. crabroniformis, très-renflés á leur bout libre dans la Laphria, en massue allongée dans la Dioctria, entièrement filiformes dans le Dasypogon. Il n'est pas rare qu'un fin ligament inséré à leur extrémité les fixe à l'origine du ventricule chylifique; leur col a une teinte roussâtre constante; dans certaines conditions physiologiques, la forme normale de la grlande est tellement changée par la supersécrétion de la salive, que cet organe est méconnaissable: c’est ainsi que dans deux 
dissections du Crabroniformis jai rencontré, au lieu d'un boyau allongé, une poche tout à fait pyriforme.

Lie tube alimentaire est bien plus long que celui des familles précédentes, car il a de trois à cinq fois la longueur de l'insecte; sa texture est généralement délicate et fragile. L'osophage a constamment, comme le col salivaire, une teinte roussâtre qui ne tient pas aux contenta. J'ai constaté dans le Crabroniformis une forme et une structure particulières de ce conduit. Peu après sa sortie du suçoir, il présente une contracture légère, mais constante, qui semble destinée à retenir momentanément l'aliment, peut-être pour la déghutition; à son insertion au ventricule, il s'implante an centre d'un disque orbiculaire qui est inférieur, de manière à être invisible au-dessus. Cette espèce de bulbe aplati, dont la figure donne une juste idće, a la texture du ventricule et non celle de l'oesophage. La tunique interne de ce dernièr est d'un roux pâle, d'une consistance un peu élastique, d'un tissu fibreux où la lentille microscopique décéle des stries transversales d'une extrême finesse, très-serrées, mais non régulièrement continues. comme, par exemple, celles en spirale des trachées; ces stries sont parfois interrompues. La tunique externe est blanchâtre, contractile, et ses lambeaux, à un fort grossissement, ont des plissures transversales qui ne sont peut-être que l'empreinte des stries annulaires dont il vient d'être fait mention.

Je suis très-porté à croire que cette organisation de l'œsophage est commune à tous les Asiliques; il est vraisemblable, vu la contracture antéricure et le mode d'insertion postéricure, que l'aliment doit y séjourner et y subir, avant de passer dans le ventricule, une espèce de trituration. Cet organe, que je n'ai rencontré dans aucun autre Diptère, serait jabot par sa position, gésier par sa texture.

J'ai longtemps cru que les Asiliques étaient tous dépourvus de panse, et ce trait négatif avait lieu de m'étonner dans des insectes d'aussi grande taille et si vigoureusement constitués. Je la découvris enfin, en 1837 , dans les deux espèces de Dasypogon; 
mais ayant depuis lors dirigé scrupuleusement et à de nombreuses reprises mes investigations anatomiques vers ce seul but dans le Crabroniformis, qui est un géant parmi nos espèces indigènes, je n'ai jamais pu y en apercevoir le moindre vestige, non plus que dans le Senex et le Laphria. Cet organe aurait-il échappé à ces mêmes yeux qui l'ont si positivement démêlé dans les entrailles du frêle cousin et de la minime psychode? C'est au moins douteux. Mais, est-ce donc la première fois que l'on voit la nature ne pas s'astreindre à nos systèmes, ne pas souscrire à nos idées législatives? Ne peut-elle pas, dans une série d'êtres oú il y a conformité d'habitudes, de traits extérieurs et même de structure intérieure, supprimer un organe sans compromettre l'existence? Que d'exemples n'en trouvons-nous pas dans l'immensité de l'échelle zoologique! Tout bien considéré, je m’inclinerais volontiers à croire à l'absence réelle de la panse dans les Asilus, et il se pourrait que cette structure singulière de l'œsophage signalée plus haut fût un trait anatomique propre à ce genre et peutêtre une compensation du défaut de la panse. Remarquons encore, à l'appui de l'exclusion de cet organe dans les Asilus, que dans les Dasypogon, où son existence ne saurait être révoquée en doute, il est, à raison de son peu de développement et de sa fragile texture, en quelque sorte rudimentaire. La panse y est placée tout à fait au-dessous du canal digestif, sur la couche épiploïque intermédiaire à ce dernier et ău chapelet ganglionnaire; son réservoir est une poche très-simple, peu expansible, oblongue dans le $D$. teulonus, ovoïde dans le $D$. punctatus.

Le ventricule chylifique est ou profondément échancré et bilobé, comme dans les Asilus, ou à lobes prolongés en véritables bourses ventriculaires, ainsi qu'on le voit dans les Laphria, Dioctria, Dasýpogon; l'extrémité de ces bourses a souvent un fin ligament suspenseur qui se fixe dans la tête. A son entrée dans l'abdomen, le ventricule offre une dilatation de forme et de grandeur variables, marquée de bandeleites transversales; enfin, il s'atténue en un très-long boyau filiforme récurrent, c'est-d̀-dire rebroussant en 
avant sous la dilatation ventriculaire pour retourner en arrière : ce boyau, habituellement vide, offre une certaine analogie avec le jejunum des vertébrés.

Les vaisseaux hépatiques, à bouts flottants et à insertions isolées, ont sept à huit fois la longueur du corps et une coulcur qui varie depuis le jaune et le brun jusqu'au rouge briqueté et au diaphane. Les insertions dans le Laphria sont rapprochées par paires, sans être confluentes.

L'intestin est d'abord filiforme, puis se dilate en un reclum oblong ayant quatre boutons charnus, orbiculaires dans le Crabroniformis et le Laphria, cinq dans le Senex 'et les Dasypogon.

Appendice. - Les larves des Asiles (que je ferai connaître dans un mémoire particulier) ont un canal digestif proportionnellement bien moins long que celui de l'insecte ailé, puisqu'il ne dépasse pas la longueur du corps. Les glandes salivaires sont deux boyaux allongés assez gros; il n'y a ni panse ni bourses ventriculaires.

CHAPITRE II. APPAREIL GENITAL.

ARTICLE I"r APPAREIL GẺNITAL MâLE.

Dans leur situation normale, les diverses parties constitutives de cet appareil sont entassées pêle-mêle au bout de l'abdomen et semblent ne former qu'une seule masse informe; mais avec cette dose de patience qui ne doit jamais abandonner les microtomistes, on parvient à les isoler, ainsi que les représentent mes figures.

Dans les Asilus et le Das. teutonus, les testicules consistent chacun dans un enroulement oblong des replis spiraux d'un seul vaisseau spermifique filiforme, brun ferrugineux, dont le bout libre est plus ou moins renflé en massue. Ce vaisseau, déroulé, a 
près de deux fois la longueur de l'insecte, et ses replis sont maintenus en tire-bouchon par de nombreuses trachéoles.

Les testicules de la Laphria ne ressemblent pas du tout, en apparence, à ceux des Asilus; mais quand on pénètre leur structure intime, on leur trouve une admirable conformité. Un véritable scrolum, parfaitement analogue à ceux que j’ai fait connaître dans heaucoup d'Hyménoptères', enveloppe et cache complétement les deux testicules; ce scrotum, arrondi.ou parfois obtusément quadrilatère, a une teinte rouillée ou fauve, ct est formé par une fine Lunique adipo-membraneuse. Si l'on y fait bien attention, on y verra une ligne médiane longitudinale déprimée, un indice d'une division en deux parties égales, une sorte de raplé qui annonce l'existence sous-jacente des deux testicules. Quand on affranchit ceux-ci de leur tunique scrotale, on voit que chacun d'eux est formé, comme dans les Asilus, par les replis spiroïdes d'un seul vaisseau séminifique, dont la longueur égale environ celle de l'in secte, et qui est blanchâtre.

Le conduit déférent, dans les divers Asiliques, n'est que la conlinuation à peine atténuće et décolorée du vaisseau séminifique.

II n'y a qu'une paire de vésicnles séminales, capillaires, diversement reployées, entortillées en une agglomération aplatie dans les Asilus, ou, déroulées, elles ont cinq ou six fois la longueur de l'insecte; moins fines et à peine de la longueur du corps dans les Laphria et les Dasypogon.

Le cunal éjaculatcur est, dans les Asilus, long, filiforme, phus gros que les vésicules séminales, comparativement beaucoup plus court dans les Laphria et les Dasypogon.

L'armare copulatrice du Crabroniformis est habituellement apparente au bout de l'abdomen; vue par sa face dorsale, elle semble consister seulement en un forceps robuste, oblong, corné, châtain clair, un peu velu, dont les branches sinueuses se terminent en pointe mousse creusée en cueilleron, et font la tenaille entre elles;

1 Rech. anat. sur les Orth. IIymén. Névropt. (Mémoires de I'Institut; 18 í.) 
vers sa base, se voit une pièce subquadrilatère veluc, noire sur les côtés, pâle au centre, ayant en dessous un panneau semi-coriacé velu. C'est entre celui-ci et la pièce subquadrilatère que je suppose l'anus. Envisagée par sa face ventrale, elle offre, indépendamment des branches du forceps : $1^{\circ}$ une pièce basilaire brune, semi-lunaire, concave en arrière; $2^{\circ}$ une volselle de deux pièces triangulaires velues à leur base, et à une arête interne; $3^{\circ}$ le foarreau de la verge, formé par deux baguettes allongées et adossées; $4^{\circ}$ une lame noire sortant de l'extrémité du fourreau et terminée par un trident.

- L'armure copalatrice de la Laphria est noire, luisante, saillante au bout de l'abdomen, même dans l'insecte mort et desséché; lorsque les pièces qui entrent dans sa composition sont fermées, sa configuration générale est ovale-suljconoïde. Comme clle est d'une structure fort compliquée, on risquerait de mal saisir et le nombre et les connexions des pièces constitutives, si l'on ne soumettait pas cette armure à une macération préalable qui tend à les désunir un peu. C'est après une semblable préparation que je l'ai décrite et figurée.

Le forceps forme la partie dorsale de l'armure : ses branches sont oblongues, robustes, glabres, presque droites, ì extrémité tronquée un peu relevée; une articulation linéaire les unit à une pièce basilaire qui semble faire corps avec elles. C'est à la faveur de cette articulation que les branches du forceps peuvent exercer. durant l'acte copulatif, les divers mouvements de préhension; à la base interne de ces branches s'implante un pinceau arqué de quatre ou cinq soies longues et roides, conchées dans le repos de l'organe.

Les pièces basilaires, dont je viens tout à l'heure d'indiquer les connexions avec le forceps, sont presque carrées, bordées de poils assez longs, échancrées en avant en demi-cercle: une sorte de coin carré enclavé entre leurs bases les unit l'une à l'autre.

La volselle, placée en arrière de ce coin carré, se compose de deux pièces brunes ovalaires el d'un harpon intermédiaire pareil- 
lement brun, formé par l'adossement de deux baguettes; l'une de celles-ci se termine par une dilatation carrée un peu crochue en dessous, le bout de l'autre est un hameçon acéré avec une petite dent aiguë à sa base interne.

A la partie inférieure de l'armure se voit une pièce réceptaculaire noire, ovale, velue, bombée en dessous, concave en-dessus, pour loger le fourreau, arrondie en avant, échancrée en arrière avec les angles de cette échancrure prolongés en un faisceau de quatre ou cinq soies roides arquées; entre ces faisceaux est une plaque transversale noirâtre, cambrée, au bord postérieur de laquelle s'insèrent deux appendices spatulés, noirâtres, velus.

Le véritable fourreau de la verge est caché dans l'excavation de la pièce réceptaculaire; il est gros, comme bulbeux, brusquement atténué en arrière, formé de plusieurs pièces étroitement unies et terminé par une double pointe acérée, sinuée. Qui nous révélera la part respective de la multitude de ressorts de cette ingénieuse machine copulative lorsqu'elle est appelée à fonctionner?

L'armure du D. tcutonus est loin de ressembler ni pour sa forme, ni pour sa composition, à celle du Laphria: vue par-dessus, elle paraît arrondie, petite et composée de trois compartiments ou panneaux principaux ovalaires convexes, hérissés de poils; l'intermédiaire est moins grand et presque triangulaire. Chacun des latéraux émet en arrière un crochet noirâtre à deux branches divergentes ou opposées entre lesquelles est une petite saillie articulée; de ces branches, l'une, externe, est pointue, arquée; l'autre, interne, obtuse, droite. Entre ces crochets, qui forment la pince et font l'office de forceps, se voient deux pointes crochues destinées à combiner avec les crochets précédents leur action préhensive et comparables à la volselle. La pièce réceptaculaire est creusée en tuile et son extrémité est tronquée et velue. 
ARTICLE II.

APPAREIL GÉNITAL FEMELLe.

Les ovaires des Asiliques sont allongés, maintenus en place, soit par le ligament suspenseur, soit par de nombreuses brides trachéennes. Des gaînes ovigères innombrables, à six ou sept loges, et terminées par un ovulaire allongé et grêle, les hérissent de toutes parts, excepté dans un ruban médian de leur face inférieure. Le calice est centrảl ou inférieur, le col court, ainsi que l'oviducte. Les xufs à terme sont ovales oblongs, blanchâtres dans le Crabroniformis, bruns ou noirâtres dans le Laphria et le Dioctria.

La glande sébifique a une structure toute particulière; l'organe sécréteur se compose de trois vaisseaux simples capillaires d'une consistance élastique, roulés en replis concentriques et tellement adhérents les uns aux autres par une membrane hyaline, qu'on dirait parfois qu'ils ne constituent qu'un seul filet. Chacun d'eux est aussi long que l'insecte, ils sont roussâtres vers leur extrémité, seulement dans le Crabroniformis, vers leur insertion dans le Laphria; ceux du D. teutonus sont roux dans toute leur étendue, avec l'extrémité noirâtre et leur origine renflée, comme bulbeuse. Les réservoirs séminaux sont, dans le Crabroniformis, deux longs boyaux filiformes subdiaphanes plus ou moins boursouflés; ils sont, au contraire, courts, ovalaires ou spatulés dans le Laphria, en forme de vésicule atténuée en col capillaire dans le $D$. teutonus; j’ai posilivement constaté dans ce dernier, que les deux cols des réservoirs s'unissent pour former un canal unique fort court implanté sur l'oviducte.

\section{FAMILLES DES EMPIDES ET DES CYRTIDES.}

Quoique la forme et la structure du corps du Cyrlus semblent le rejeter loin des Empis, cependant les entomologistes praticiens habitués à l'étude des physionomies des insectes ne sauraient 
disconvenir de l'air de famille que donne à ces deux types leur figure de bécasses. Dans le seul but de concentrer mon texte, je les comprends dans une même description.

Les espèces disséquées sont:
1. Empis unicolor. Mace.
4. Empis stercorea. L.
2. - L. livida. L.
5. Ramphomyia sulcuta FaLL.
3. - lutea. Meig.
6. Cyrlus acephalus. Latr.

\section{CIAPITRE PREMER.}

APPAREIL DIGESTIF.

J'ai souvent confirmé l'observation de M. Macquart sur les habitudes insectivores des Empis, dont les mâles, à l'exomple des Tabanus, se nourrissent du nectar des fleurs; l'étude des contenta du canal digestif du Cyrlus m'a aussi donné l'assurance que ce Diptère vit du pollen des étamines.

Les glandes salivaires ont une configuration très-différente de celle des Asiliques; ce sont deux vaisseaux d'une ténuité capillaire, d'une longueur qui sonvent atteint deux fois celle de l'insecte; dans le $E$. stercorca, elles sont labituellement fléchies au milien et récurrentes.

Le tube alimentaire a une fois et demie à deux fois la longueur du corps. Le réservoir de la panse est bilobé dans le Cyrtus simple, oblong ou allongé dans les Empis; il atteint presque le bout de l'abdomen dans le E. livida, il s'avance moins dans le E. stercorea. il a plus d'ampleur dans le $E$. unicolor. Le ventricule chylifique débute par deux bourses oblongues obtuses; il est uniformément cylindroïde et droit dans les Empis, dilaté avant sa terminaison dans le Cyrlus.

Les vaisseaux hépatiques ont dans ce dernier genre deux canaux chólédoques, et c'est là un trait anatomique différenticl avec les Empis; où les insertions sont isolées; ces vaisseaux sont fort longs, peu ou point variqueux, blanc jaunâtre ou'verdàtre. 
L'intestin, d'abord filiforme, a un reclum ovoïde dans le $E$. livida, allongé dans le $E$. lutea globuleux dans le Cyrtus, avec quatre boutons charnus conoïles.

\section{CHAPITRE II.}

APPAREIL GÉNITAL.

ARTICLE I $]^{\text {er }}$

APPAREIL GÉNITAL MÁLE.

Les lesticules sont ovoïdes ou sphéroïdaux, d'un brun marron ou chocolat dans les Empis, le Ramphomyia, incolores dans le Cyrtus. Les conduits déférents des Empis sont courts, grêles, bruns; ceux du Cyrtus, aussi fins et plus longs, reçoivent, peu après leur origine, un court boyau latéral.

Les vésicules séminales sont dans les Empis au nombre de trois paires, et leur configuration est tellement variable suivant les dispositions génératives, qu'il m'est arrivé de croire, malgré ma rigueur accoutumée dans la détermination préalable de l'espéce, que je m'étais mépris sur celle-ci. Dans le stercorea, la paire principale, celle qui reçoit directement les conduits déférents, est, dans la condition de turgescence, plus ou moins ventrue, courbée en crosse dirigée en avant; dans la condition contraire, elle est petite, filiforme, déjetée en arrière. Dans le $E$. lutca, ces mêmes vésicules, bien plus longues que dans le $E$. stercorea, sont filiformes, avec un grand renflement ovoïde. Les deux autres paires de vésicules sont, dans le $\boldsymbol{E}$. stercorea, fort longues, capillaires plus ou moins boursouflées, l'une d'elles, dans le E. lutca, est fort courte et rudimentaire. Je n'ai trouvé dans le Ramphomyia que deux paires de ces vésicules, l'une filiforme, l'autre terminée par une utricule ovoïde; il n'y en a dans le Cyrtus qu'une seule paire, allongée, courbée en crosse, atténuée en arrière.

Le canal éjaculateur est fort court dans les Empis et Ramphomyia, assez long et filiforme dans le Cyrtus. 
L'armure copulatrice des Empis est d'une curieuse complication; mais l'imperfection de mes notes ne me permet pas d'en esquisser la description. Celle du Cyrlus a un forceps ì branches grêles courbées en bas et largement ouvertes; elle a aussi une volselle de deux pièces ovalaires velues et un fourrean de la verge noir, allongé, refendu à son extrémité, rostriforme.

\section{ARTICLE II.}

IDPAEIL GENITAL FEMEII,E.

J'ai liguré et je vais décrire cet appareil dans le $\boldsymbol{E}$. livida; j’ai anssi étudié celui du $L$. unicolor, qui lui ressemble.

Les ovaircs, semblables à ceux des Asiliques, forment deux grappes oblongues de gaines ovigères courtes, quadriloculaires, innombrables, mais non très-pressées, affectant une disposition sériale. Le col est tubuleux, médiocrement long; l'oviducte fort court. Les œuf's sont ovales obtus, assez gros, blancs.

La glande sébifique diffère beaucoup de celle des familles précédentes; au lieu d'orbicelles, il n'y a ici qu'une seule vésicule sphérique, blanchàtre, au centre de laquelle s'implante un col efférent capillaire, long et flexueux. Dans le Ramphomyia, je vois un seul orbicelle rond à large centre noir, à conduit efférent apical, courbé en anse et plus ou moins boursoutlé; les réservoirs séminaux sont, comme dans les Asiliques, formés par un double vaisseau simple, filiforme, semi-diaphane, atténué vers son insertion; jai constaté dans le Ramphomyia que les cols de ces réservoirs s'insèrent de chaque côté de la terminaison du conduit efférent de la glande.

L'oviscapte se compose de deux stylets droits, grêles, acérés, cornés comme dans les Tipulaires, flanqués à leur base par les tentacules valvaires noirs, un peu arqués. La forme et la structure de cel oviscapte annoncent que les Empis enloncent leurs oufs dans quelque milieu résistant. 
FAMILLES DES BOMBYLIERS ET DES ANTHMACIENS

Déjà à l'article de l'appareil respiratoire, j’ai témoigné mon étonnement de ce que malgré leur vol rapide et soutenu, malgré leur bourdonnement aigu et saccadé, les Bombyles étaient privés de ces aérostats abdominaux qui sont l'apanage ordinaire des insectes qui ont de semblables habitudes, jai fait remarquer aussi que les Anthrax, qui planent silencieusement, avec leurs longues ailes étendues, étaient munis de ces aérostats.

Mais si les traits extérieurs et quelques habitudes distinguent évidemment les Bombyliers des Anthraciens et légitiment, pour l'étude, leur séparation en deux familles, il n'en est pas ainsi de leur organisation viscérale; celle-ci présente une conformité qui m'a déterminé à confondre l'exposition de mes recherches. Toutefois, jai déjà fait ressortir leur différence, sous le rapport du système nerveux.

Espèces disséquées :

BOMEYLIERS.

1. Bombylins posticus. F.

2. minor. L.

3. major. L.

4. cruciatus. 'T.

5. ctenopterus. MEIG.

6. Usia cenea. Latr.

7. Phtiria minuta. Melg.
ANTHRAGIENS.

1. Antlirax jacchus. F,

2. - punctata. T.'

3. - flava. Horrs.

4. venusta. MEIG.

\section{CHAPITRE PREMLR.}

APIAREIL DIGESTIF.

Les Bombyliers et les Anthraciens se nourrissent du pollen des fleurs,

Les glandes salivaires, à peine de la longueur du corselet, sont 
cylindroïdes, en massue dans quelques Bombyliers, terminées dans le $A$. jacchus par un renflement ovoïde, fléchies à angle aigu vers le milieu dans le $A$. venusla. Le col est presque nul dans les Bombyliers, bien prononcé dans les Anthraciens, et c'est là un trait caractéristique dans les deux familles.

Ramdohr (l.c.p. 184), qui a décrit el figuré le canal digestif du $B$. major, que j’ai aussi disséqué, n'a pas connu les véritables glandes salivaires, et a pris pour telles les bourses ventriculaires défectueusement représentées.

Le tube alimenlaire a près de trois fois la longueur de l'insecte dans les Bombylius, deux fois seulenent dans les Phtiria el Usia, un peu moins dans les Anthrax. Le réservoir de la panse est en besace, avec les poches oblongues dans les Bombylius et Anthrax, arrondies dans les autres genres. Ce réservoir m'a souvent offert une pulpe jaune forméc par le pollen des étamines : cette pulpe est safranée dans la Phtiria, que je soupçonne sucer les anthères du serpolet. Le ventricule chylifique débute par deux bourses oblongues, plus ou moins boursoullées ou ridées; en entrant dans l'abdomen, il offre une, quelquefois deux dilatations, puis il redevient cytindroïde.

Les vaisseaux hépaliques sont blanchâtres ou jaunâtres, plus ou moins variqueux : deux d'entre eux distribuent leurs flexuosités en avant, et les deux autres en arrière; mais ils présentent, quant a leur mode d'insertion, quelques différences suivant les genres: ainsi, dans les Bombylius et Anthrax, ils sont rapprochés par paires i l'endroit de leur implantation sans être confluents; dans l'Usia, où ils sont proportionnellement plus gros et moins longs, ils se réunissent d'abord deux d̀ deux, puis les deux cols aboutissent à un seul canal cholédoyuc. Je sens le besoin de soumettre cet insecte à de nouvelles vivisections, à cause de ce mode d'insertion si exceptionnel dans la famille.

L'intestin est filiforme, le reclum, ovale ou oblong avec deux paires de boutons charnus, arrondis, peu saillants; je n'en ai pas aperçu dans le $A$. jacchus. 


\title{
CHAPITRE II.
}

\author{
APPAREIL GENITAL.
}

ARTICLE I ${ }^{\text {er }}$

IPPAREIL GÉYITAL MÂtE.

Il se fait remarquer par sa petitesse et diffère essentiellement de ceux des familles qui précèdent. Je prendrai pour type de ma description celui du B. posticus.

Les testicules, ovoïdes et d'un brun ferrugineux, dégénèrent insensiblement en un conduit déférent filiforme, coloré comme eux et de leur longueur au moins. Les vésicules séminales forment une masse agglomérée, au milieu de laquelle se perdent les conduits déférents, rapprochés et contigus. On prendrait, au premier coup d'œil, cette agglomération pour une seule grosse vésicule; mais un scalpel adroit et heureux y démêle deux pelotons égaux contigus, formés chacun par les entortillements inextricables d'un vaisseau unique aussi fin que le brin d'un cocon et dont la longueur dépasse celle de l'insecte; le canal éjaculatcur, presque entièrement caché sous ce peloton, est à peu près droit et de la longueur du conduit déférent.

L'armure copulatrice, qui se dérobe à l'œil au milieu de la fourrure du bout de l'abdomen, peut facilement être mise en évidence par une compression expulsive bien ménagée. Le forceps en forme la partie la plus apparente : il ent ovale oblong, coriacé. velu, plus large et bombé à sa base, déprimé et atténué à l'extrémité de ses branches, qui est obliquement tronquée; une plaque triangulaire, placée comme un coin entre les bases du forceps, est l'analogue de la pièce basilaire. La volselle, qui est inféricure, tantôt dépasse et tantôt n'atteint pas les extrémités du forceps; elle est formée de deux pièces adossées roussâtres, terminées par un crochet ployé habituellement en dessous, mais 
qui se redresse dans l'acte copulatif. Ce crochet est affilé à sa pointe et hérissé de soies piquantes dirigées d'arrière en avant, et de deux ou trois épines au côté interne. Le fourreau de la verge, situé cntre les branches de la volselle, est allongé.

Dans l'Usia, les testicules et les conduits déférents ressemblent à coux du Bombylius; mais les vésicules séminales, loin d'être confondues en une seule agglomération, sont très-distinctes et formées pour chaque côté par un seul vaisseau très-flexueux ou reployé.

Les organes sécréteurs et conservateurs du sperme ressemblent, dans le Phtiria, à ceux de l'Usia; mais les vésicules séminales sont fort courtes, rélléchies en arrière et renflées vers leur insertion au canal éjaculateur.

Les testicules du $A$. venusta sont oblongs, non colorés; les conduits déférents capillaires, de leur longueur; les vésicules séminales, tout aussi grêles et entremêlées. Le canal éjaculateur est plus court que les conduits déférents.

\section{ARTICLE II.}

APPAREIL GÉNITAL FEMELLE.

Avant d'exposer les organes essenticls de ce sexe, je ferai connaître la curieuse structure de l'extrémité de l'abdomen des Bombyles femelles, et en particulier celle du B. major. Par ume compression expulsive graduée, on voit se dérouler des parties qui, dans le repos, restent profondément engagées dans les derniers segments : c'est d'abord une large ceinture dorée et soyeuse de poils fins, serrés, dans le sens de la longueur du corps, formant en arrière une frange courbée; ensuile, apparait une sorte de segment ayant au milieu un écusson brunâtre, glabre, et sur les flancs un duvet doré pâle, fin, moelleux, couché. Il y a de chaque côté du bord postérieur de ce segment un peigne à une douzaine de dents longues, fines, noires, droites et parallèles, 
qui, au microscope, sont autant de baguettes cornées, un peu en massue. Deux panneaux largement tronqués, finement velus, et entre lesquels on parvient à déterminer la hernic ou du rectum ou du vagin, forment la dernière pièce mise en évidence. Il ne nous est pas donné d'assigner à ces pièces leurs attributions physiologiques, soit dans l'acte copulatif, soit pour la ponte.

Les ovaires du $B$. crucialus, dans une gestation avancée, forment charm une grosse grappe globuleuse de gaînes ovigères innombrables, uniloculaires, convergentes; le calice est central, le col tubuleux, de la longueur de l'ovaire, l'oviducte court, terminé par deux tentacules vulvaires oblongs; obliquement tronqués; les $\propto u / s$, globuleux dans les gaînes, sont ovalaires dans le calice.

L'appareil sébifique (et séminal) a une complication désespéraite pour le physiologiste. J'avoue et tout mon embarras pour la désignation de quelques-unes de ses parties constitutives et toute mon incertitude pour les véritables attributions fonctionnelles. Il m'a paru s'insérer à la face inféricure de l'origine de l'oviducte. Il y a trois orbicelles à centre brun, ovoïdes, contigus, se continuant en arrière en un col efférent qui offre an milieu un trait longitudinal brun, inclus, analogue au point central de l'orbicelle; les trois cols aboutissent à une souche commune fort courte. Dans le $B$. crucialus, le col a une dilatation qui ne s'observe pas dans le B. major, et le trait brun inclus est infiniment plus court dans la première de ces espèces et le $B$. minor que dans la seconde. Dans le $B$. major, dont l'orbicelle est rond et forme la base d'un cône, ce trait brun se termine en avant comme en arrière par un bourrelet transversal qui dépasse un peu son diamètre; mais ce que jai bien constaté, en déchirant le tissu de l'organe, c'est que ce trait communique, par un conduit incolore plus fin que lui, et à l'onbilic brun de l'orbicelle et á son insertion postérieure: la texture contractile de l'enveloppe extérieure du col efférent se imanifeste, au microscope, par de légers festons de ses bords.

Cette glande offre d'autres modifications spécifiques clans le $B$. ctenopterus; les orbicelles à centre brun et arrondis ont des cols 


\section{RECHERCIES ANATOMIQUES ET. PIIYSIOLOGIQUES}

trois fois plus longs que dans les précédents, courbés en anse et tellement accolés ensemble, qu'ils en imposent pour un seul conduit; mais on parvient a les isoler : ils n'ont pas le trait brun et ils se renflent en arrière.

Les réservoirs, que jhésite à appeler séminaux, sont au nombre de deux paires, au moins dans le $B$. crucialus: l'une, que jappellerai essentielle, parce qu'elle s'observe dans toutes les espéces de Bombylius, est vésiculaire, ovoïde ou pyriforme, blanchâtre, a parois asśez épaisses, mais souples, atténuéc en arrière pour l'insertion brusque d'un col capillaire; oblongue dans le B. major et le $B$. ctenoplerus, plus longuement atténuée dans le $B$. minor : ces vésicules correspondent à ce que, ailleurs, j’ai appelé provisoirement réservoirs séminaux. L'autre paire, constatée dans le B. cruciatus, consiste en deux longs vaisseaux simples, plus fins qu'un cheveu, reployés et perdus au milieu de la pulpe adipeuse du bout de l'abdomen. Quelles sont donc les attributions physiologiques de ces filets capillaires reployés, qui, d'ordinaire, sont sécréteurs? Judicent periliores!

Les ovaires de l'Usia et du Phtiria sont ovalaires et non globuleux, avec un col tubuleux bien plus marqué que clans les Bombyles, surtout dans le Phtiria; les gaînes ovigères sont bi ou triloculaires dans le premier genre, uniloculaires dans le second: les aufs à terme, dans celui-ci, sont ovales et d'un roux pâle. Il y a dans l'Usia trois orbicelles à centre brun et deux réservoirs séminaux longs, filiformes.

\section{FAMILLES DES THÉRÉVIDES ET DES LEPTIDES.}

J'examinerai en commun leur splanchnologie dans le seul but d'abréger mon texte, car, en entomologie, ce sont deux groupes bien distincts. J'ai disséqué les :

1. Thereva plebeia. L.

2. bipunctuta. MEIG.

3. nobilitata. T.
4. Thereva confinis. Meig.

5. Leptis tringaria. L.

6. Chrysopila aurata. Mace. 


\section{CHAPITRE PREMIER.}

APPAREIL DIGESTIF.

Les Thérévides, que l'on trouve souvent posés à terre dans les expositions chaudes, à la manière des Asiles, vivraient, dit-on, du pollen des fleurs; tandis que les Leptides, habituellement perchés sur les plantes, chasseraient de très-petits insectes pour les sucer.

Quoi qu'il en soit de l'espèce de leur nourriture, leur appareil digestif est organisé sur le même plan que celui des familles précédentes, et les différences sont pen remarquables ${ }^{1}$.

Les glandes salivaires, qui ont échappé à Ramdohr, ne diffèrent pas de celles des Bombyliers; elles sont ou ovales-oblongues ou en massue, et ne dépassent pas le milieu du thorax: leur col est assez long, mais moins dans le Chrysopila.

Le tube alimentaire n'a pas plus de deux fois la longueur de l'insecte; le réservoir de la panse est bilobé. Ramdohr, qui l'appelle sac pharyngicn, l'a représenté à tort comme simple et allongé : je crois qu'il s'en est laissé imposer par une partie du vaisseau dorsal. Les bourses ventriculaires sont oblongues et opposées, subgranuleuses dans les Thercva; le ventricule offre une dilatation constante à son entrée dans l'abdomen; les vaisseaux hépaliques ont leurs insertions rapprochées par paires, mais non confluentes, et leurs quatre bouts sont flottants, contre l'assertion erronée de Ramdohr, qui les représente sous la forme de deux anses. L'intestin est renflé à son origine dans le Leptis et le Chrysopila, et non dans les Thereva, ou il est filiforme; le rectum a quatre boutons

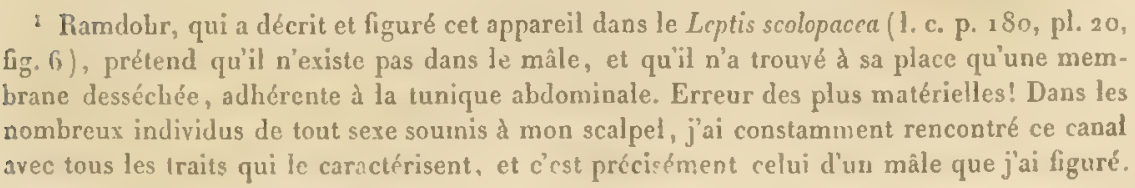

11. 
charnus ronds, a paires assez distantes, et se termine dans les femelles par un col plus long.

\section{CHAPITRE II.}

APPAREIL GÉNITAI.

ARTICLE I

IPPIREIL GÉNITIL M ILE.

Les testicules, plus ou moins rapprochés et ensevelis dans la pulpe adipeuse sont ovoïdes ou sphéroïdes, blancs dans le Leplis, d'une tcinte roussàtre dans les Thereva. Le conduit déférent est presque nul dans ces derniers, où il ne semble que le col du testicule; il a dans le Leptis une ténuité capillaire et une longueur qui surpasse sept a huit fois celle de ce dernier organe : il offre dans cet insecte une disposition anormale fort difficile à constater. D'abord, collés sous les vésicules séminales, ces.conduits sont récurrents et s'unissent en un col commun fort court, qui s'abouche ou conflue avec l'extrémité antérieure de ces vésicules; celles-ci, couchées sur la ligne médiane, sont droites, courtes, grêles, adossées l'une à l'autre : la figure exprime assez bien cette disposition pour ne pas y insister. De plus habiles que moi découvriront peut-être que la confluence antérieure des vésicules n'est qu'une simple contiguité. Les vésicules séminales des Thereva sont simples, filiformes, blanches, et reçoivent les conduits déférents, comme à l'ordinaire, avant leur réunion pour former le canal éjaculaleur : celui-ci est aussi long et plus grêle que les vésicules dans le Thereva, court dans le Leplis.

L'armure copulatrice du Thereva, à peine apparente dans la profonde échancrure demi-circulaire du dernier segment de l'abdomen, est fort compliquée. On y distingue : $1^{\circ}$ une pièce basilaire formée de trois plaques coriacées, velues, dont les latérales sont terminées en pointe pilifère, et l'intermédiaire oblongue; 
$2^{\circ}$ un forceps corné à branches atténuées et fortement crochues en dedans; $3^{\circ}$ deux slylets cornés, bruns, tronqués, terminés par des soies roides; $4^{\circ}$ une volselle de deux pièces ovales-oblongues; $5^{\circ}$ le fourreau de la verge, placé au centre de l'armure et oblong.

La pièce basilaire du Leptis est une plaque unique, noire, cornée, en forme de large triangle; le forceps a des branches conoïdes, noires, avec un crochet terminal articulé, peu arqué, brun, semblable à celui de la mandibule d'une Arachnide; te fourreau de la verge est assez petit; la volselle, tout à fait inférieure, se termine par deux tentacules articulés, ovales, ciliés en dehors.

AR'TICLE II.

APPAREIL GÉYITAL FEMELLE.

Les ovaires sont deux grappes oblongues ou ovalaires, blanchâtres, de gaînes ovigères innombrables et triloculaires dans plusieurs Thereva, au nombre de neuf ou dix seulement et sur deux rangées dans la $T$. confinis, subbiloculaires dans le Leptis, où elles sont moins serrées et parfois disposées d'une manière distique; le calice est central; le col et l'oviducle sont fort courts dans le premier genre, assez longs dans le second; tes cufs sont ovalesoblongs, blancs.

La glande sébifique des Thereva a trois orbicelles dépourvus de centre noir et globuleux. Je n'en ai vu que deux dans la T. confinis, espèce qui offre au centre de l'appareil une bourse ovoïle, membraneuse, comparable à la poche copulatrice d'Audouin. Les cols efferents sont capillaires et l'intermédiaire cst presque nul; les réservoirs sont deux boyaux allongés en massue, terminés en arrière par un col Jjrusquement capillaire plus court qu'eux; la vulve est flancquée à droite et à gauche par un panneau coriacé, hérissé en dehors de piquants et de soies roides.

Cette même glande présente dans le Leptis des différences marquées. Les orbicelles sont remplacés par trois courtes digitations 
blanchâtres, disposées en triangle, et tout aussitôt conlluentes pour donner naissance à un long conduit efférent unique, capil-laire; Ies réservoirs sont deux boyaur filifurmes ausi loness gue le corps de l'insecte et remplis d'une pulpe blanche comme de l'amidon : ils s'atténuent en arrière pour s'insérer sur l'oviducte. Les trois derniers segments de l'abdomen, à cause de leur largeur décroissante, semblent faire l'office d'oviscapte; les tentacules vulvaires se composent, pour chaque côté, de deux articles: l'un, basilaire ovale-oblong; l'autre, terminal globuleux, comme brièvement pédicellé.

\section{FAMILLE DES DOLICHOPODES.}

Cette famille, fondée par Latreille et conservée par Fallen et Meigen, est une des plus naturelles de l'ordre.

Les espèces disséquées sont :

1. Dolichopus nitidus. Meig.

2. chalybaus. id.

3. Porphyrops diuphanus. T.

\section{CHAPITRE PREMIER.}

APPAREIL DIGESTIF.

Il est des Dolichopodes qui semblent ne se nourrir que des sucs excrétés par les feuilles et d'autres qui font la chasse aux petits insectes pour les sucer.

Les glandes salivaires, d'une petitesse presque rudimentaire, mais parfaitenent organisées, sont ovoïles et débordent à peine dans leur position normale le contour occipital du crâne, en sorte qu'il faut briser celui-ci pour les mettre en évidence: leur col est plus court qu'elles.

Le tube alimentaire n'est guère plus long que l'insecte; le réservoir de la panse est trilobé ou à trois poches: celles-ci ne sont 
pas toujours faciles à constater. J'ai souvent trouvé l'une d'elles vide et affaissée, de manière qu'alors le réservoir ne semblait que bilobé. La représentation de ces divers états me dispense d'autres détails. Le ventriculc cliylifique débute par une configuration, une composition intéressantes à constater, parce qu'clles forment la transition, le chaînon des familles précédentes aux suivantes. Tout en conservant les bourses ventriculaires, il offre à son origine un godet orbiculaire, comme les Muscides. Ces bourses sont allongées, opposées, plus ou moins arquées; le ventricule se continue ensuite en un tube filiforme.

Les vaisseaux hépatiques, d'uine finesse extrême, sont ici, comme dans les Leptides, à quatre bouts flottants et à insertions isolées.

L'intestin est d'abord grêle; le rectum n'a que trois boutons charnus : ils sont arrondis et entourés d'un cerceau comme cartilagineux.

\section{CHAPITRE II.}

IPPAREIL GÉNITAL。

ARTICLE I ${ }^{\mathrm{er}}$.

APPAREIL GÉNITAL MÂLE.

Les testicules sont assez gros, vu la petitesse de l'insecte, subglobuleux ou ovoïdes, distincts, quoique rapprochés, tantôt châtains, tantôt à peine lavés de brun; le condait déférent est plus court que le testicule et plus ou moins boursouflé; le canal éjaculateur, aussi court et plus fin que ce conduit, s'insère un peu latéralement à l'armure copulatrice : il y a une paire de vésicules séminales filiformes plus ou moins reployées.

L'armure copulatrice, simplement couchée sous l'abdomen et pouvant être facilement mise en évidence, a été depuis longtemps décrite par De Géer dansle $D$. ungulalus. Les diptérologistes mo- 


\section{RECHERCIES $\triangle$ NATOMIQUES ET PHYSIOLOGIQUES}

dernes ont fondé sur son existence et sur ses configurations des caractères propres à la distinction des espèces. Le corps de l'armure est, dans le $D$. nitidus, de texture tégumentaire, cylindroïle, glabre, noirâtre. Un oil scrupuleux le trouve composé de deux pièces étroitement unies: l'une, basilaire, bien plus courte, arrondie, convexe, enchåssée obliquement ou latéralement dans l'autre; celle-ci échancrée en arrière et débordée par deux lamelles ovalaires, blanches, de texture scarieuse ou sèche, garnies au bord externe, qui est convexe, de cils noirs, longs, plus ou moins courbés, les uns simples, les autres (postérieurs) divisés en deux dès leur base, qui est une souche plus ou moins prononcée: le bord interne de ces lamelles est presque droit et garni d'une courte villosité microscopique.

\section{ARTICLE II.}

APPAREIL GÉNITAL FEMELLE.

Les ovaires se présentent sous l'aspect de deux grappes ovalaires et déprimées de gaines ovigères subbiloculaires, au nombre d'une trentaine environ, insérées à la paroi supérieure seulement, de manière que le calice est inféricur; le col, qui est tubuleux, et l'oviducle, sont de la même longueur. Les œufs sont ovales-arrondis, blancs.

La glande sébifique a une structure très-différente de celle des familles précédentes; elle consiste en une seule vésicule ovoïde terminée en arrière par un col efférent capillaire, d'une longueur démesurée, enroulé en nombreuses circonvolutions, quatre ou cinq fois plus long que le corps de l'insecte, et renflé avant son insertion à l'oviducte. Les verres amplifiants font reconnaître dans ce col un vaisseau inclus roussâtre, avec quelques légères dilatations. Les réservoirs séminaux sont, comme dans les familles précédentes, deux boyaux cylindroïdes, plus ou moins courbés en anse et atténués en un col capillaire moins long qu'eux.

L'oviducte s'engage dans un oviscapte composé de quatre tuyaux 
engainés, d'autant plus étroits qu’ils sont plus postérieurs; le dernier offre, au microscope, une série pectinée de dix dents cornées, et en dessous deux tentacules valvaires d'un seul article oblong, droit, noir : ce peigne ou râteau de l'oviscapte annonce une manœuvre particulière pour enfoncer les œufs. De Géer nous apprend que c'est dans la terre que vivent les larves du dolichope. (Mém. vol. VI, p. 194.)

\section{FAMILLE DES SYRPHIDES.}

J'ai disséqué les espèces suivantes:

1. Chrysotoxum arcuatum. Meig.

2. Volucella zonaria. Id.

3. Eristalis arbustorum. T.

4. - - tonax. T.

5. - sepulchralis. 'T'.

6. Tylota segnis. Meig.

7. Syrilla pipiens. MACQ.

8. Rhingia rostrata. T.
9. Milesia crabroniformis. 'T

10. Syrphus pyrustri. T.

11. rosarum. 'T.

12. - nectareus. T.

13. Spherophora tóniall. MEIG.

14. Cheilosia mulabilis. Mace.

15. - scutellata. MeIg.

16. Chrysogaster metallica. Id.

\section{GHAPJTRE PREMER.}

APPAMEIL DIGESTIY.

Les Syrphides se nourrissent tous du pollen et du nectar des fleurs, ainsi qu'il conste de l'étude de leurs habitudes et de celle des contenta de leurs organes digestifs.

Les glandes salivaires, plus développées et surtout plus longues que dans les trois familles précédentes, sont filiformes, plus ou moins reployées, généralement de la longueur du corps, mais plus courtes dans le Syrph.pyrastri; elles s'étrécissent plus ou moins brusquement en un col capillaire qui, dans quelques espèces, et notamment dans le Volucella, a une certaine longueur.

Le tube alimentaire a deux ou trois fois la longueur de l'insecte dans les Chrysotoxum, Er. sepulchralis, Xylota, Syritta, Rhingia, 
Syrph. pyrastri et rosarum, Sphcerophoria, Cheilosia, Chrysogaster, quatre fois environ dans les Volucella, Er. Icnax et arbustorum, Milesia, Syrph. nectareus. La panse a son réservoir en bissac; ses poches peuvent être égales ou inégales, séparées par un profond sinus.ou presque confondues en une seule, distendues ou plissées, ou festonnées, quelquefois, comme dans le Spharophoria, formées d'un groupe de vésicules arrondies; le col de la panse est, dans le Rhingia, proportionnellement plus gros et un peu renflé à son insertion à l'osophage.

L'origine du ventricule chylifique a constanment deux bourses appendiculées, c'est-ì-dire formées chacune d'une grande et d'une petite digitation : ces bourses sont oblongues ou allongées, plissées ou festonnées, ou granuleuses, suivant certaines conditions digestives. C'est la plus antéricure des deux digitations qui est ordinairement la plus courte, et elle est d'une petitesse rudimentaire dans le Syrph.pyrastri et le Xylota : je ne connais qu'une seule espèce où les deux soient presque égales entre elles, c'est le Rhingia. Quant à leur mode d'insertion, il a lieu de chaque côté de l'origine du ventricule par un col parfois d'une telle brièveté, 'qu'il est impossible de le constater, el les bourses paraissent alors sessiles, comme dans l'Er. tenax. Il m'a scmblé que dans les Volucella, Er. sepulchralis et arbuslorum, et Rhingia, le col des bourses s'implantait à la terminaison de l'oesoplage, et non à l'origine du rentricule; mais je sens la nécessité de diriger des investigations plus scrupuleuses sur ce point d'anatomic. Le plus souvent, il y a continuité ou communication directe entre les digitations du même côté, qui ne sont séparées que par un étranglement.

Ramdohr (l.c. p. 77) s'est encore mépris sur la nature et les fonctions de ces bourses, qu'il a prises pour des vaisseaux salivaires, tout en disant qu'ils s'insèrent à l'origine de l'estomac, circonstance anatomique évidemment contraire à la fonction connue des glandes salivaires.

Le ventricule chylifique, long, cylindrö̈de, glabre, etrangle an détroit thoraco-abdominal, présente à peine quelques légères mo- 
difications, suivant les espèces; il est sensiblement plus court et simplement flexueux dans les Syrphus, tandis que dans les autres il se reploie en une circonvolution ou en une grande anse. Celui du Rhingia et du Chrysotoxum se fait remarquer par une grande dilatation ovoïde à l'entrée de l'abdomen, sans préjudice de la circonvolution : cet organe débute ordinairement par un godet orbiculaire mal circonscrit, qui n'existe pas dans le Rhingia.

Les vaisseaux hípaliques sont unis ou variqueux, diaphanes, blanchâtres, grisâtres; jaunes, bruns ou même noirâtres (Er.tenax) : leur insertion a lieu ou par deux canaux cholédoques fort courts, ou isolément, comme dans les Syritta et Xylota.

L'intestin a d'abord une portion grêle filiforme, flexucuse ou replové: le reclum s'atténue en un col plus ou moins long : il a Le plus sourent quatre houtons charmus, ronds ou concïdes. Le Volucella est jusqu'à ce jour le seul Syrphide où je n'aie trouvé aucune trace de ces bontons: je ne saurais me rendre raison de ce trait négatif exceptionnel.

\title{
CHAPITRE II.
}

\author{
APPAREIL GÉNITAL.
}

ARTICLE I ${ }^{\text {en }}$.

APPAREIL GÉNITAL MÂLE.

La composition de cet appareil s'éloigne peu, quant aux parries principales, de celle qui s'observe dans les familles que nous venons de passer en revue; toutefois, nous allons trouver dans la plupart des Syrphides un organe qui n'a pas d'analogue dans ces dernières, et qui constitue un des faits anatomiques les plus caractéristiques des Syrphides, c'est l'exisłence d'un réservoir spermatique distinct des vésicules séminales.

Exposons d'abord cế appareil dans le Volucella, l'un des plus gros Syrphides de nos contrées. 


\section{RECHERCHES ANATOMIOUES ET PIYSIOLOGIQUES}

Les testicules, petits relativement à la taille de l'insecte et revếtus d'une tunique brune, ont une configuration singulièrement diverse, suivant leur degré de turgescence séminale : ainsi, vous les trouverez tantôt oblongs, cylindroïles ou comprimés au milieu, tantôt ovoïdes ou pyriformes. Les conduits déférents, bruns aussi, capillaires et un peu moins longs que les testicules, deviennent, avant leur terminaison, tellement contigus l'un à l'autre, qu'uir wil peu pratique de ces dissections délicates croirait qu'ils ne forment là qu'un seul et même conduit eourt; mais le secours de la lentille microscopique dissipe tous les doutes : ils s'insèrent à l'origine inférieure du canal éjaculateur. Les vésicules séminales, une pour chaque côté, sont filiformes, flexueuses ou reployées, trois ou quatre fois plus longues que les testicules; elles confluent en arrière pour la formation du canal éjaculateur.

Celui-ci, loin d'avoir, comme à l'ordinaire, des parois fibreuses et un diamètre filiforme, a une texture vésiculaire, une forme allongée, cylindroïde, et une grosseur insolite : il renferme un lhuide prolifique dont l'abondance modifie beaucoup sa configuration. Cet organe est, d mes yeux, un type précieux de transition qui nous prépare à trouver mieux circonscrit ce réservoir spermatique que j’ai dit être un trait anatomique des Syrphides.

L'armure copulatrice de la volucelle rappelle par sa composition, et surtout par son étui articulé, celle de la panorpe; mais au lieu d'être replié en dessus, comme dans cette dernière, cet étui est, dans l'état de repos, ployé et reçu dans une excavation particulière du bout inférieur de l'abdomen : il est roux (noir dans le V. inanis), composé de quatre articles assez grands, ovalaires ou subquadrilatères, velus et mobiles les uns sur les autres, comme des vertèbres caudales. Le forceps, qui termine l'étui, a ses branches cornées, brunes, velues, courbées en crosse à leur extrémité, qui est un peu épaissic et faisant la pince : entre ces branches, se voit une sorte de volselle, mi-partie coriacée ct membraneuse, très-velue et partagée. en deux portions égales par un intervalle linéaire. Le fourrean de la verye, placé au-dessous de la volselle, est corné et 
d'une couleur rembrumie, dont la nnance varie; les baguettes, qui sont glabres, se dilatent en une raquelte arrondie armée en dessous d'un crochet à double griffe se rattachant à une tige appliquée contre la baguette; la base interne de cette tige a trois ou quatre dents microscopiques, et son milicu une isolée. Tout à fait au-dessous de tout l'appareil, il y a une pièce cornée, noirâtre, glabre, profondément échancrée à sa base el munie, au centre de son extrémité tronquée, d'une apophyse fourchue. Cette pièce inarticulée est l'analogue de celle que j'ai appelée hypotome dans les Hyménoptères.

Cette complication du forceps de la volucelle fait supposer dans l'acte de la copulation des manouvres curieuses, dont la constatation directe est destinée, je crois, à demeurer bien longtemps un mystère pour nous.

Les testicules du Chry'sotoxum sont subglobuleux, d'un brun pâle, un peu plus gros que ceux de la volucelle; les conduits déférents, capillaires et bruns, sont distincts jusqu'ì leur insertion a un conduit commun droit, plus gros mais aussi long qu'eux et pareillement brun. Ce dernier conduit, qui semblerait l'analogue du réservoir spermatique, loin d'être formé par la confluence des vésicules, serait au contraire reçu dans l'embranchement de celles-ci. Cette disposition anormale, ce mode de connexion, la teinte et l'aspect non vésiculaire du conduit, me font naître des scrupules sur ses attributions, et incliner à croire que je n'ai pas assez multiplié les autopsies. Les vésicules séminales, au lieu d'être longues et flexueuses comme dans la volucelle, sont allongées, droites, plus ou moins fusiformes; le canal éjaculateur serait fort court, le forceps n'est pas précédé d'un étui articulé, et sen branches se terminent par une pointe droite.

Les testicules de l' $E$. sepulchralis sont assez gros, ovoïdes, d'un brun rouillé, les conduits déférents, aussi longs qu'eux, sont jaunâtres et non bruns, droits, bulbeux à leur origine; en approchant du point de leur insertion au réservoir spermatique, ils deviennent contigus et adhérents, ce qui rend encore plus fondés 
les scrupules et les doutes émis à l'article du Chrysoloxum; ils s'implantent à la partie postérieure et inférieure de ce réservoir. Les vésicules séminales, semblables à celles de la volucelle, et par leur forme et par leur longueur, s'insèrent à côté des conduits déférents; le canal éjaculatcur est grêle, filiforme, aussi long que le réservoir, dont il n'est que le col tubuleux.

Le bout de l'abdomen du mâle de notre éristale est très-obtus, convexe, velu, et se courbe un peu en bas pour devenir le réceptacle ou le couvercle de l'armure. Celle-ci est transversalement ovalaire, non symétrique, beaucoup plus bombée du côté droit, glabre. Les branches du forceps sont presque droites, brunes, comprimées et se terminent par deux dents bien prononcées, dont linterne est plus courte.

Le plus vulgaire des Eristalis, le E. tenax, va encore nous offrir dans les formes et les connexions de cet appareil des traits spécifiques d'anatomic: les lesticules, fort petits et relégués sous le rectum, sont ovales, bruns, sessiles ou presque sessiles sur les bords du réservoir spermatique, de manière que le conduit déférent semble nul. Ce réservoir utriculeux est ovalaire et du volume du testicule; il ne reçoit pas, comme dans l'espèce précédente, les insertions des vésiçules séminales, et il ne forme pas directement le canal éjaculateur; il s'insère à la paroi supérieure de celui-ci après la confluence des vésicules; ces dernières, moins longues que dans le $E$. sepulchralis, forment au-dessous du réservoir le canal éjaculateur.

Nous trouvons dans le $E$. arbustorum le mème plan d'organisation que dans le E. tenax, mais avec des modifications; les teslicules, oblongs, subcylindroïdes et bruns, sont en partie cachés par le réservoir spermatique, a la face inferiemre el postirieure duquel ils sinsèrent par des conduits déférents incolores, fins et si courts, qu'ils ont à peine le quart de la longueur de ces glandes; les vésicules séminales, en massue allongée, s'implantent distinctement en arrière des conduits déférents sous le réservoir; celui-ci est sphéroïdal, et se continue en un conduit tubuleux, grêle, filiforme, qui n'est que le canal éjaculatcur. 
Dans la plus grande espèce européenne de Milcsia, les lesticules, remarquables par leur longueur filiforme, sont reployés et ordinairement un peu rénflés en massue à leur bout libre; ils sont d'un brun chocolat. Les conduits déférents, colorés de même et plus courts qu'eux, s'adossent ensemble avant leur implantation au bout antérieur du réservoir; les vésicules séminales, grĉles comme un fil, reployées et de la longueur de l'insecte, s'insèrent à cóté et en dehors des conduits déférents. Le réservoir spermatique a une configuration bien différente de celle des Eristalis : il est allongé, fusiforme, et son bout antéricur, replié en crosse, reçoit à la convexité de celle-ci les insertions des organes précédents. Le canal éjaculatcur ne serait ici, comme dans beaucoup d'autres Syr̂̀phides, que le col de ce réservoir.

L'étude de ces organes dans le Xylota va nous fournir une des nombreuses preuves des ressources inépuisables de la nature dans leurs formes et leurs combinaisons, pour remplir un même but. Les testicules, en massue oblongue, arquée et brune, dégénèrent brusquement en conduits déférents colorés de même, fins comme un cheveu, et s'unissant bientôt, ou plutôt s'adossant, pour ne former en apparence qu'un cordon unique, bien plus long que leur portion dégagée, reployé ou llexueux, s'insérant en arrière et en dessous du réservoir spermatique. Les vésicules séminales sont longues, capillaires, reployées ou agglomérées et se fixent à côté des conduits déférents; le réservoir spermatique est en massue allongée; le canal éjaculateur n'en est non plus ici que te col atténué.

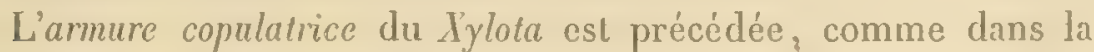
volucelle, d'un étui articulé mais composé seulement de deux articles d'un noir violet, velus, fléchis l'un sur l'autre, et dont la convexité du premier termine l'abdomen; le second, courbé en bas et en dedans, abrite sous sa voûte les divers instruments copulateurs; ces derniers sont $: 1^{\circ}$ deux écailles basilaires dont l'une est le réceptacle, l'opercule de pièces qui ne s'observent pas dans l'autre $; 2^{\circ}$ un forceps à branches brunes inégales, se croisant 
dans leur jeu, l'une plus grosse et velue; $3^{\circ}$ en dehors du forceps, un corps moins corné, obtus, velu, d'un gris sale: c'est peut-être le fourreau de la verge; $4^{\circ}$ une sorte de volselle compliquée dont je n'ai pas assez étudié les pièces constitutives.

Les testicules du Syritta ressemblent à ceux du Milesia; par leur gracilité filiforme, mais ils sont un peu moins longs; les conduits déférents, fins, courts et parfois boursouflés, s'insèrent isolément, et non adossés, au tiers antérieur et inférieur du réservoir spermatique; les vésicules séminales, grêles comme les testicules, mais moins longues et incolores, ont leurs insertions à côté de celles des conduits déférents. Le réservoir spermatique, allongé et subfusiforme offre, à son tiers antérieur, une légère contracture où s'implantent les organes précédents; il s'atténue en arrière en un filet capillaire qui est le canal éjaculateur. L'armure copulaIrice a, comme celle du Iylota, un étui biarticulé; l'article basilaire, plus grand, reçoil latéralement le canal éjaculateur; les branches du forceps et le fourreau de la verge sont conme dans la volucelle.

Le Rhingia offre des formes très-insidieuses dans cet appareil; il faut une cerlaine habileté dans ces vivisections et avoir bien présente cette composition anatomique dans les gemres voisins pour s'y reconnaître. Les lesticules, d'un lorun rougeâtre, petits. globuleux, contigus entre eux, sont sessiles sur l'aire du réservoir spermatique; lorsqu'on cherche à les isoler pour en saisir les connexions, on leur découvre un col d'une extrême brièveté. qu'il faut regarder comme un conduit déférent rudimentaire. En arrière et un peu au-dessous des testicules, une loupe attentive aperçoit une paire de très-petits gloljules subdiaphanes et sessiles: ce sont les vésicules séminales. Lè réservoir spermatique est une grande utricule sphérö̈dale qui supporte, comme jé viens de le dire, tous les autres organes; le canal éjaculatcur est aussi le col du réservoir. L'armurc copulalrice est précédée, non pas de deux articles comme dans le Xylota, mais d'un seul placé obliquement à l'axe du corps et velu. Les branches du forcens, robustes et cam- 
brées, sont formées de deux pièces unies par une articulation linéaire transversale et sont tronquées au bout.

Si nous consultons ce même ordre d'organes dans le Syrph.rosarum, où il n'existe aucune trace de réservoir spermatique, et où l'armure ;opulatrice débute par un étui de quatre articles, nous verrons que le genre Syrphus doit, dans la série générique, être plus rapproché de la volucelle et même la précéder. Les testicules de ce diptère sont globuleux, d'un brun clair; les conduits déférents, à peu près de leur longueur et capillaires, s'insèrent, non pas au réservoir spermatique, qui n'existe pas, mais aux vésicules séminales, qui sont allongées et atténuées en avant; le canal éjacu-. lateur, qui résulte évidemment de la confluence de ces dernières, est grêle et assez court.

Les deux espèces du genre Cheilosia manquent aussi de réservoir spermatique. Les testicules du C. mutabilis, petits, subglobuleux et d'un fauve vif, ont des conduits déférents grêles, fauves aussi, et s'adossant, avant leur insertion, de manière à simuler un conduit unique; les vćsicules séminales sont globuleuses, bien plus grandes que les testicules, et confluentes pour la formation d'un canal éjaculateur filiforme, plus long qu'elles; les testicules du C. scutcllata sont, au contraire, beaucoup plus grands que les vésicules et munis d'un conduit déférent bien plus court qu'eux; les vésicules séminales sont ovoïdes.

ARTICLE II.

IPPAREIL GÉNITAL FEMELLE.

Il n'y a pas autant de variétés ou de modifications organiques dans l'appareil de ce sexe que dans celui du mâle. Je me bornerai à décrire en détail celui du Volucella.

Les grappes qui constituent ses ovaires sont ovales ou ovalesoblongues, blanchâtres, garnies de gaines ovigères modérément pressées, biloculaires, terminées par un petit ligament; le calice est 
central, et si, lorsqu'il renferme beaucoup d'œufs, on renverse l'ovaire, on aperçoil un ruban médian plus ou moins large, dégarni de gaînes, mais inégal ou bosselé par la présence intérieure des œufs; dans la condition de virginité, le ruban est remplacé par une fine rainure linéaire, de laquelle semblent partir à droite et à gauche les gaines ovigères, disposées obliquement comme les

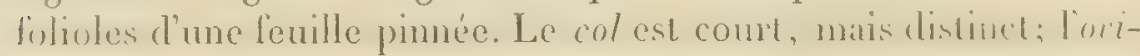
ducte, tubuleux; les oufs sont ovales dans la gaîne, oblongs dans le calice.

L'appareil sébifique (et séminal) se compose : $1^{\circ}$ de trois orbicclles à centre noirâtre, groupés en triangle, munis d'un trèslong col efférent capillaire; $2^{\circ}$ de deux réservoirs séminaux sous la forme de deux boyaux filiformes atténués à leur insertion à l'oviducte. L'oviscaple, caché dans l'était de repos, est à découvert lors de la ponte, et on peut lc mettre en évidence par une compression expulsive ménagéc: on se convainct alors qu'il se conpose de trois tuyaux engaînés et rétractiles, dont les deux premiers sont bordés de jaune et dont le dernier, plus petit, ovaletriangulaire, se termine par deux tentacules vulvaires ovales-oblongs, velus, d'un seul article.

Les ovaires des autres Syrphicles ne diffèrent guère, que par lc nombre des locules, des gaînes ovigères; il y en a quatre dans le E. tenax, six ou sept dans le Sphcerophoria, etc.

Les réservoirs séminaux présentent dans le $\boldsymbol{E}$. tenax une forme, une structure dont, jusqu'à ce jour, les Diptères ne m'ont ollert d'exemple que dans la famille des Pupipares (Hippobosca) ; au lieu des deux boyaux simples, ordinaires, on trouve, dans cette espéce, deux arbuscules trés-rameux, blancs, surtout dans une gestation avancée, à rameaux capillaires recourbés ou entortillés, aboutissant à un tronc pareillement capillaire, qui s'insère à l'oviducte, conjointement avec les cols des orbicelles. Ces arluuscules rappellent, par leur configuration seulement, les glandes vénénifiques des Hyménoptères, notamment celles du Larra ${ }^{1}$, et

1 Recherch. anat. elc. (Miem. de l'Instit. 1841, pl.8, fig. 106.) 
aussi les organes des sécrétions excrémentitielles de quelques carabiques.

\section{FAMILLE DES SCÉNOPINIENS.}

Déjà, aux chapitres des appareils nerveux et respiratoires, j’ai parlé de l'espèce d'anomalie que forme, dans les Diptères, le Sccnopinus, et de l'embarras qu'il cause pour son poste définitif dans le cadre entomologique. Latreille, qui, le premier, en forma un genre particulier, le colloqua d'abord dans la famille des Muscides, entre les genres Ochtera et Pipunculas; plus tard, il le refoula dans les Dolichopodes, après le Platypeza. Meigen jugea mieux l'originalité de ce genre en créant pour lui seul la famille des Scénopiniens, que M. Macquart plaça comme tribu à la tête de l'indigeste famille des Athéricères. Stephens (Syst. catal. of Brit.) le rejeta encore bien plus loin dans la série des genres, en le plaçant entre le Chlorops et le Mosillus, dont il a un peu les habitudes tranquilles.

La composition de son système nerveux, qui a cinc ganglions abdominaux, la longueur de son canal digestif, qui dépasse peu celle du corps; l'existence des bourses ventriculaires; enfin, la forme et la structure de ses antennes doivent rapprocher le Scenopinus des familles qui ont un rang plus élevé dans l'ordre, et, comme je l'ai déjà insinué, il offre plusieurs liens de parenté avec les Thérévides.

La seule espèce que j’aie disséquée est :

Scenopinus fenestrulis. Latr.

\section{CHAPITRE PREIIER:}

APPAREIL DIGESTIF.

Quelle est la nourriture des Scénopiniens? On ne nous l'a pas encore appris. L'espèce qui a servi à mes recherches passe sa 
paisible existence sur les vitres de nos maisons, où, sans doute, elle vit, comme la mouche domestique, de quelques atomes mucilagineux ou sucrés, ou d'imperceptibles immondices répandus çà et là. J'ai aussi rencontré abondamment sur les fleurs de persil un Scenopinus, peut-être le Sc. niger, qui se nourrissait du pollen.

Les glandes salivaires sont capillaires, simples, et ne franchissent pas le détroit thoraco-abdominal.

Le tube alimentaire est à peu près droit; la panse a un réservoir simple et ovoïde quand il est distendu, festonné ou lobé quand il est vide : son col est assez gros, comparativement à celui d'autres Diptères. Le ventricule chylifique a deux bourses ventriculaires oblongues : il se rentle à son entrée dans l'abdomen pour s'étrécir de nouveau. Les vaisscaux hépatiques, plus gros et moins longs que dans la plupart des genres voisins, sont capillaires, d'un jaune blanchâtre, se rapprochent par paires, et confluent à leur insertion, mais sans canal cholédoque; il y a là une souche sessile, ainsi que l'exprime la figure. Ceux qui'se portent en avant m'ont paru plus longs que ceux dirigés en arrière; l'inteslin est grêle; le rectum est ellipsoïdal avec deux seuls boutons charnus orbiculaires à sa partie antérieure el supéricure.

\section{CHAPITRE 11.}

APPAREIL GENITAL.

ARTICLE $I^{e r}$.

APPAREII. GENITAL MÂLE.

Les testicules, rapprochés et assez grands, vu la petitesse de l'insecte, et d'un brun chocolat, sont ovoïdes, un peu atténués en arrière, et presque sessiles sur la vésicule séminale; le conduit déférent est, par conséquent, nul, et il faut bien considérer comme rudiment de ce conduit l'étrécissement du testicule; les vésicules 
séminales sont deux longs boyaux capillaires qui confluent en arrière pour la formation du canal éjaculateur, qui est pareillement capillaire et plus long que le testicule.

L'armure copulatrice, au lieu d'être enchatonnée sous l'abdomen, comme dans les Syrphides et les Muscides, est placé aprés le dernier segment dorsal qui lui sert d'opercule. Par une compression expulsive, on voit s'étaler, comme par ressort, quatre larges panneaux ovalaires ou obtusément quadrilatères noirs, velus, loordés, au côté interne, qui est légèrement échancré, de longs cils roussâtres: les panneaux supérieurs font l'office de forceps et les inférieurs celui de vôlselle; à leur centre, on découvre une pièce plus petite qui n'est, sans doute, que le fourrcau de la verye.

ARTICLE II.

APPAREIL GÉNITAL FEMELLE.

Les ovaires du Scenopinus sont deux grappes oblongues de gaînes ovigères innombrables, tri ou quadriloculaires, terminées par' un ligament; le calice est central, le col assez long, l'oviducte à peine de sa longueur; les œufs à terme sont ovalaires, roussâtres ou d'un blond foncé.

La structure et la composition de l'appareil sćbifique et séminal ont aussi leur trait d'originalité. Je ne saurais m'empêcher de considérer comme les analogues des orbicelles deux capsules, une de chaque côté, ovales oblongues, oviformes, d'un gris obscur, entourées, au microscope, d'une tunique hyaline, adhérentes par leur bout antérieur à la vésicule du réservoir séminal; cette adhérence, qui n'est certainement pas accidentelle et qui ne peut se rompre que par un certain effort, est destinće à fixer ces grosses et lourdes capsules pour les empêcher de ballotter et de se meurtrir : c'est là une explication des plus rationnelles. Au bout postérieur de chacune de ces capsules s'implante brusquement un conduit efférent, fin comme un brin de soie, élastique, enroulé 
en boucles nombreuses, et d'une longueur qui dépasse dix ou douze fois celle du corps de l'insecte; soumis à la plus forte lentille du microscope, il offre un tube inclus, comme les conduits excrétcurs en général, et avant son insertion à l'oviducte, il se dilate un peu.

Le réservoir sćminal, car il n'y en a qu'un, mériterait tout aussi bien, je crois, le nom de poche copulatrice; c'est une vésicule subglobuleuse, a parois pellucides, située au centre de l'appareil, terminée en arrière par un col capillaire qui se fixe à l'extrémité postérieure de l'oviducte.

L'oviscapte est presque nul : il y a deux tentacules vulvaires d'un seul article oblong, un peu arqué, velu.

\section{Quelques mots sur le Lonchoptera et le Platype-a.}

Pour me conformer à la série générique de M. Macquart, que j’ai adoptée, je vais exposer ce que des dissections trop peu multipliées m'ont appris sur le Lonchoptera fossarum ${ }^{2}$ et le Platypeza holosericea, Meig. deux genres contigus dans cette série.

$1^{\circ}$ Lonchoptera. Avant d'aborder l'anatomic, voici les obsertions que m'a mis à même de faire l'étude de trois on quatre espèces de ce genre de Diptères paludicoles.

Tête subglobuleuse et non large et déprimée, comme l'avance M. Macquart, bien détachée du corselet et hérissée en dessus et en dessous de soies rares et longues; ocelles petits disposés. en triangle sur une éminence arrondie; y'cux ronds subhémisphériques, séparés par un très-large front; antennes courtes subturbinées, dirigées en avant. Le troisième article, presque globuleux et non comprimé, enchatonné dans le second, soit apical, long,

1 Espèce nouvelle, voisine, mais distincte, des $J_{\text {. }}$ riparia et lacusiris, Meıg. En voici le signalement:

Capite albuilo-flacesccnte, subscriceo, nigro-piloso, regione occllari antennis palporumquc apicibus nigris; thoracis pallidi fuscia dorsali aliaque laterali abbreviata nigris, sculcllo pallido, dorso nigro; abdomine supra nigro, lateribus subtusane pallido; pedibus pallidis, tarsis anticis nigris. Hab. in fossis. Long. 3 millim. 
villosule : palpes allongés et relevés, comme dans les Tabanus; balanciers, remarquables par leur forme en massue allongée, bien saisic par Meigen.

Par la forme de ses glandes salivaires, par la longueur de son canal alimentaire, par l'absence de bourses ventriculaires, caraclère anatomique d'une grande valeur, par ses vaisseaux hépatiques à deux canaux cholédoqques; enfin, par la privation de ballons trachéens dans l'abdomen, le Lonchoptera appartient à la grande famille des Muscides acalyptérées, et se rapprochẹ singulièrement des genres Tetanocera et Helomyza, dont il partage les habitudes et la physionomie.

$2^{\circ}$ Platypeza. La présence de deux bourses ventriculaires, - la longueur du tube digestif, qui dépasse à peine celle de l'insecte, les insertions isolées des vaisseaux hépatiques, sont autant de traits anatomiques importants" qui éloignent le Platypeza du Lonchoptera, pour le rapprocher du Scenopinus, et pour le colloquer avec ce dernier dans le voisinage des Thérévides.

J'ai décrit et figuré la larve fongivore et les métamorphoses du Plalypeza holosericea. (Annal. des sc. nat. mars 1840.)

\section{FAMIJLE DES CONOPSAIRES.}

Je me suis déjà expliqué au chapitre du système nerveux sur la fusion des Conopsaires et des Myopaires en une seule et même famille : la splanchnologie est toute à l'appui de cette fusion.

Les espèces dont j’ai étudié l'anatomie sont :
1. Conops flavipes. L.
4. Myopa ferrugineu. F.
2. —— rufipes. T.
5. Slachynia meridionatis. Uisco.
3. Incera. Meig. 


\section{CHAPITRE PREMER.}

APPAREIL DIGESTIF.

On trouve souvent les Conopsaires sur les fleurs, et il est vraisemblable qu'ils se nourrissent du pollen.

Les glandes salivaires présentent des configurations différentes suivant les genres : dans les Conops, elles sont uniformément capillaires et assez longues pour pénétrer dans la cavité abdominale; elles revêtent dans le Myopa la forme de bourses conoïdes, effilées en arrière, avec un col efférent capillaire, moins long qu'elles et brusquement inséré à leur gros bout; celles du Stachynia ressemblent à une capsule ovale oblongue suspendue à un col qui a trois ou quatre fois au moins sa longneur.

Le tube alimentaire a une fois et demie a deux fois la longueur de l'insecte; le réservoir de la panse, logé dans le bout renllé de l'abdomen, est simple, ovoide ou globuleux dans le cas de sa distension. Le ventricule chylifique n'offre plus ici la moindre trace de bourses ventriculaires; il débute constamment par un godet, sphéroïdal dans les Conops, orbiculaire et ombiliqué dans les Myopaires. Il est droit et cylindroïde. Je lui ai trouvé dans le Stachynia quelques dilatations ou boursouflures, sans doute accidentelles.

Les vaisseaux hépatiques assez gros, médiocrement longs et id bouts flottants, sont grisâtres ou blanchâtres et confluent de chaque côté par paires à un canal cholédoque, assez long dans le Conops, plus court dans le Myopa et encore davantage dans le Stachynia.

L'intcstin est filiforme et flexueux. Le rectum est remarquable par l'épaisseur et la consistance calleuse de ses parois. Il est globuleux dans le $C$. rufipes où j'ai constaté l'existence de quatre boutons charnus à sa moitié antérieure, oblong dans le lacera et dans les autres genres, sans que j’aie pu y découvrir aucun indice de ces boutons. 


\section{CHAPITRE II.}

APPAREIL GENITAL.

La configuration bizarre de l'alsdomen des Conops devait faire supposer des manœurres singulières pendant l'acte copulateur. J'ai été assez heureux pour être témoin oculaire, en juillet 1838 de l'accouplement du Conops rufipes, dont j'avais renfermé dans un bocal de verre plusieurs individus pour mes dissections. Le mâle monte sur le dos de la femelle, qu'il tient embrassée par le corselet. Il l'excite par des attouchements, par de petits coups répétés de ses pattes. En même temps il fait glisser en se reculant, son abdomen sur celui de la femelle, il le recourbe en bas en agitant alors et la trompe et les palpes, et en faisant frémir ses balanciers. La femelle, d'abord assez froide, finit par répondre à ce prélude de caresses et se met à l'unisson de l'orgasme sexuel. Elle déroule son abdomen, de manière qu'au lieu d'ètre courbé en dessous it se relève en dessus pour s'unir et s'emboîter étroitement avec celui du mâle. Il se fait aussitôt une rétroversion du mâle conıme fontles chiens, et les deux abdomens sur une mème ligne semblent n'en former qu'un seul. J'ai représenté cette union des deux abdomens. Le couple demeura ainsi attaché pendant un quart d'heure.

Il parait que plusieurs assauts ou plusieurs copulations doivent avoir lieu pour une complète fécondation, ou qu'il y a des pontes successives, car, en disséquant une femelle qui venait de s'accoupler sous mes yeux, jai trouvé les gaînes ovigères et même les cols des ovaires avec des oufs à terme. Cette femelle était certainement à même de pondre. 
ARTICLE I $\mathbf{I}^{n}$.

APPAREIL GÉNITAL MILE.

Los testicules des Conops (lacera), placés à la partie postérieure de la cavité abdominale, sont formés chacun par les circonvolutions agglomérées d'un seul vaisseau spermifique filiforme, brun (roussâtre dans le rufipes), plus long, quand il est déroulé, que le corps du Diptère. Ces organes s'insèrent directement aux points de confluence des vésicules séminales; ainsi, il n'y a pas de conduit déférent proprement dit, et on ne peut considérer comme tel que la partie postérieure du vaisseau testiculaire. Les vésicules séminales, filiformes comme ce dernier, mais moins longues, sont subdiaphanes et reployées. Le canal éjaculateur encore plus fin, mais du double plus court, semble un peu bulbeux à son origine.

L'armure copulatrice du C. lacera est logée sous la voûte du bout de l'abdomen. Le forceps est noir, velu, court, épais, avec l'extrémité des branches très-obtuse. Dans l'intervalle de celles-ci, on voit saillir un petit bouton, comme pédicellé, qui paraît s'ouvril dans son milieu, et que je soupçonne être une volselle. Une compression expulsive détermine l'exsertion au delà du forceps d'une pièce oblongue brune, hérissée, au microscope, de piquants trèscourts, et sur un de ses côtés une petite écaille oblongue appliquée sur sa base. C'est peut-être là un fourreau de la verge d'une structure insolite.

Les testicules du Stachynia sont aussi filiformes, reployés, en deux ou trois boucles, roussâtres, moins longs et plus épais que ceux des Conops, manquant pareillement de conduits déférents. Les vésicules séminales sont allongées, plus ou moins boursouflées et ployées sur elles-mêmes. Le canal éjaculateur ne diffère pas de celui du Conops.

Ces organes ont la même forme, la même structure dans le 
Myopa; seulement, les testicules, avant leur insertion aux vésicules séminales, sont adhérents entre eux, comme dans quelques Syrphides.

L'armure copulatrice du Stachynia offre une particularité remarquable de structure. Le forceps en fer à cheval, très-courloe et presque rond, est noir, velu. Entre ses branches est une masse oblongue, blanchâtre, charnue, dont l'axe intérieur paraît être une tige cornée, manifestée au-dchors par un trait médian noirâtre, longitudinal. Sa base est flanquée à droite et à gauche par un filet lorun recourbé, et de son extrémité sort une lame longue et élastique, plus ou moins reployéc, bordée d'un filet corné noirâtre, rappelant la langue des Apiaires, et finement velue en dessous. Cette lame, qui est vraisemblablement un fourreau de la verge analogue à celui des grandes Tipulaires et aussi à celui de quelques Muscides acalyptérées, égale en longueur la moitié du corps de l'insecte. De son bout on voit saillir par une compression expulsive une sorte de boyau charnu, qui est peutêtre le pénis.

ARTICLE II.

APPAREIL GÉNITAL FEMELLE.

Les ovaires des Conopsides diffèrent de ceux des familles précédentes surtout par le nombre déterminé de gaines ovigères et par l'absence de calice propre; ceux du Myopa sont deux faisceaux conico-turbinés, d'une douzaine seulement de gaînes ovigères allongées, multilocutaires, assez lâches, convergentes au ligament suspenseur. Le col est tubuleux, aussi long que l'ovaire et peut renfermer trois ou quatre œufs à terme, ce qui supplée le calice. L'oviducte est tout aussi grêle; mais plus court. Les œufs sont allongés, blancs.

La glande sébifique a cela de particulier qu'il y a quatre orbicelles à centre noir rapprochés etcontigus par paires. Chacune de cellesci a un seul col efférent capillaire. Les réservoirs consistent, 11. 
comme à l'ordinaire, en deux filets tubuleux, plus ou moins boursouftés. Le dernier segment de l'abdomen est grand, brun, ovale, velu, suivi d'une pièce étroite, débordée au milieu par deux tentacules vulvaires uni-articulés, oblongs, terminés par un pinceau de poils.

Les ovaines et la glande sébifique du Conops rufipes ressemblent en tout à ceux du Myopa.

\section{FAMILLE DFS OESTIRIDES 1 .}

Les OEstrides, Diptères qui altaquent nos bestiaux, et dont les larves vivent dans leurs viscères ou dans leurs divers tissus, excitaient prissamment mon intérêt et ma curiosité; mais la difficulté de se procurer pour les dissections des individus ailés vivants m'a forcé à laisser dans l'exposition de mes recherches anatomiques quelques lacunes; je ne désespère pas de les combler dans le courant de la campagne actuelle.

L'existence d'un seul ganglion rachidien et de deux ballons trachéens à l'abdomen place irrévocablement les OEstrides dans cette nation sans bornes des Athéricéres de Latreille, et en particulier à la tête de cette première division que M. Robineau Desvoidy a appelée les Myodaires calyptérées. N'est-il pas bien consolant de voir que, par un accord sublime des caractères anatomiques intérieurs avec les traits fournis par l'étude de la configuration et de la structure extérieures, ces Diplères doivent conserver le poste quiils occupent dans la série générique de la plupart des méthodes entomologiques? Mais, à l'époque où en est la science, on ne saulait se borner à les considérer, ni comme des genres de la famille (les Muscides, ni comme une tribu des Athéricères. Ils doivent

1 Depuis que mes recherches anatoniques sur les OEstricies ont été déposées au secrúlariat de l'Acadénie des sciences, et pendant l'intervalle septennal qui s’est écoulé jusqu'ì lenr publication, la science n'est pas deneurée stationnaire. Un mémoire fort remarqquable de M. le professeur Joły, de la Faculté des sciences de Toulouse, a été Jivré au monde savant en 1846. Pendant la correction de ces épreuves (novembre $\$ \$ \$ 6)$, je mettrai ì profit les faits fournis par l'habile scalpel de ce professeur. 
constituer une famille à part, ainsi que l'out bien jugé Meigen, Leach, Stephens, etc.

Les OEstrides ailés dont j'ai fait la dissection sont précisément du nombre de ceux auxquels les entomologistes, tant anciens que modernes, ont refusé une bouche, par conséquent la faculté d'avaler des aliments et de se nourrir, par conséquent un canal digestif et forcément un anus. Mais, grâce à Dieu, cette sentence n'est pas sans appel, non plus que celle qui avàit condamné le Fourmilion a ne pouvoir pas expulser le résidu de la digestion ${ }^{1}$, non plus qque l'assertion de Ramdohr, qui dénic à plusieurs insectes un tube alimentaire que j’ai trouvé parfaitement organisé. D’un autre côté, tout en disant que les cestres avaient une vie éphémère, et l'induction était de rigueur àvec une diète absolue, on leur reconnaissait un genre de vie très-actif, un bourdonnement aigu; on accordait la faculté d'engendrer à ces étalons qui, par un privilége physiologique inouï, devaient sécréter du sperme, se livrer à de vigoureux ébats, sans prendre un seul picolin, sans avaler un atome de nourriture. A tant faire que de les déshériter si injustement d'un appareil digestif, pourquoi ne pas refuser au moins aux mâles des organes reproducteurs? pourquoi ne pas déclarer les femelles à fécondité transmissible de génération en génération depuis le commencement du monde, ou fécondables par le simple contact de leurs maris impuissants? Sachez donc que les OEstrides ont un organe de la digestion, comme je le prouverai tout à l'heure.

Les espèces disséquées sont:

1. Cephalemyia ovis. MacQ.

2. OEstrus equi. Id.

3. Hypoderma bovis. Id. (larve.)

: Recherches anatomiques, etc. (Mémoircs de l'Instit.1841.) 


\section{CHAPITRE PREMIER.}

APPAREIL DIGESTIF.

La petitesse disproportionnée de cet appareil avec la taille de l'insecte, la finesse et la texture éminemment fragile des membranes digestives, témoignent assez du peu d'énergie de la nutrition dansles OEstrides. Ces circonstances rendent très-difficultueuse la dissection de cet organe, et j’ose à peine avouer que j’ai employé six heures consécutives à mettre en évidence le canal digestif d'un seul individụ de Cephalemyia. Mais enfin j'ai atteint positivement mon but.

Jusqu'à présent je n'ai pu découvrir dans les OEstrides ailés soumis à mon scalpel aucune trace de l'existence des glandes salivaires, quoique je les aie rencontrées dans quelques-unes dé leurs larves.

Le tube alimenlaire du Cephalemyia a environ une fois et denie la longueur du corps du Diptère. La panse a un col aussi gros que le ventricule, et un réservoir simple oblong, que jai trouvé rempli d'une bouillie blanchâtre. Cette dernière circonstance prouve, d'une part, que ce Diptère avale de la nourriture, qu’il a par conséquent une bouche, que tout le monde lui refuse, et de l'autre qu'il suce, non le sang, mais sans doute les mucosités ou les humeurs exsudées des brebis.

Lé ventricule chylifique présente à son origine un renflement brusque, conoïde avec une sorte de bourrelet ébauché, puis il dégénère en un tube droit d'une finesse capillaire.

Les vaissseaux hépaliques, au nombre de quatre, à bouts flottants, sont remarquables par leur grosseur, qui surpasse de beaucoup celle du ventricule; par leurs varicosités et les festons de leurs bords. Ils sont semi-diaphanes, courts comparativement à ceux des insectes en général, s'abouchent par paire à deux canaux cholédoques bien prononcés. Un développement aussi considérable des 
vaisseaux biliaires entraine, comme conséquence physiologique, une grande énergie de leurs fonctions. Ils semblent suppléer i l'exiguilté du ventricule.

L'intestin, d'un calibre bien supérieur à ce dernier, est filiforme et flexueux. Le rectum très-dilaté, presque rond, a des parois diaphanes et minces avec deux boutons charnus, arrondis ou subconoïdes. Ce rectum renferme une matière excrémentitielle, blanche comme de l'amidon. Loin de s'étrécir en arrière én un col, il s'applique au contraire largement contre la portion tégumentaire, à la surface externe de laquclle se voit l'anus. Celui-ci est bien distinct, dans la femelle, de la vulve qui l'avoisine, et qui est flanquée de deux tentacules vulvaires hérissés.

Le canal digestif de l'OEstrus est organisé sur le même plan général que le précédent, mais il est moins grêle; le plus souvent je l'ai trouvé diaphane et sans ingesta. La panse, si elle existe, m'a échappé, malgré plusicurs autopsies; le ventricule chylifique a aussi à son origine ce renflement turbiné que je viens de signaler dans le Cicphalemvia; il est variable pour son développement, et il n'est pas rare qu'il présente dans l'abdomen une dilatation; les vaisseaux hépatiques, moins variqueux, s'unissent deux à deux en un canal cholédoque court; le rectum, atténué en un col, ne m'a pas offert de boutons charnus, et il renferme aussi une pulpe fécale blanchâtre, que j’ai distinctement vue expulsée par l'anus.

Le canal digestif de l'OE. homorrhoidalis et celui de l'Hypoderma bovis, disséqués par M. Joly, ont la plus parfaite analogie avec celui de l'OE. equi.

IPPALEIL DIGESTIF DES LARVES.

Pour le complément de l'histoire anatomique des OEstrides, je vais faire connaître l'organe digestif des larves de l'Hypoderma bovis, qui acquiert jusqu'à trente-cinq millimètres de longueur, et de l'OEstrus equi. Réaumur, danș son mémoire sur les œstres du bœuf et du cheval (l.c. t. IV, p. 5o3), a épuisé tout ce qui est rela- 


\section{¿86 REGHERCIES ANATOMIQUES ET PHYSIOLOGIQUES}

lif aux formes et à la texture extérieures, au genre de vie et aux métamorphoses de ces larves: quant d̀ l'anatomic elle était toute à faire.

I.es glandes salivaires, consistent en deux bourses oblongues ou allongées, assez grosses, munies d'un col capillaire moins Jong qu'elles.

Le tube alimentaire a une longueur qui dépasse quatre fois environ celle de la larve. Il fait dans son trajet plusieurs circonvolutions sur lui-même. L'œsophage a la ténuité d'un cheveu et est assez long. La panse a un réservoir ample, bilobé et un col moins long que dans l'insecte ailé. Le ventricule chylifique débute par un renflement plus ou moins turbiné, à parois épaisses et calleuses, revêtant tous les traits d'un gésier, surtout dans l'OEstrus. Ce gésier est aplati en devant, et l'oesophage s'implante à son centre. Le ventricule se continue ensuite en un tube filiforme festonné ou boursoullé, et reployé deux ou trois fois sur lui-même.

Les vaisseaux hépatiques assez gros, d'une longucur médiocre, plus ou moins variqueux, à bouts flottants et d'un beau jaune, s'unissent par paires immédiatement avant leur insertion; mais le caual cholèdoque est imperceptible.

L'intestin ressemble pour son diamètre et ses boursouflures au ventricule. Il fait dans l'Hypoderma une ou deux circonvolutions. II est moins long dans l'OEstrus. Le rectum n'est que peu ou point marqué.

\section{CHAPITRE II.}

APPAREIL GENITAL.

ARTICLE $\mathbf{l}^{\mathrm{er}}$.

A P PAREIL GÉNITAL. MÂLE.

Je ne l'ai encore étudié que dans l'OEstrus.

Les testicules sont assez gros, sphéroïdaux, revêtus d'un enduit enfumé, excepté à leur bout antérieur, qui est dénudé et blanchâtre. Les conduits déférents, à peine plus longs que les testicules, 
dont ils sont la continuation atténuée, ont. la même nuance rembrunie. Il y a une paire de vésicules séminales de la longueur du testicule, utriculaires, arrondies, munies d'un col capillaire. Elles s'insèrent aux conduits déférents plutôt qu'elles ne le reçoivent. Le canal ćjaculatcur, grêle comme un fil, forme dès sa naissance deux anses rapprochées et se renfle à sa terminaison.

L'armure copulatrice, logée et enchatonnée sous le bout de l'abdomen, est arrondie. Le forceps a ses branches brunes, velues, oblongues, médiocrement arquées, obtuses à leur extrémité, et débordées à leur base par un crochet aigu. Dans le vide du forceps parait une volselle à branches plus petites et encore moins arquées que les précédentes: Le fourreau de la verge s'accompagne de deux baguettes cornées courtes. Le pénis, dont j’ai procuré l'exsertion par une compression expulsive, est cylindroïle, charnu et blane.

ARTICLE II.

1PPAREIL GENITAL FEMELI,F.

En automne, par un temps orageux, je saisis sur mon cheval une femelle d'OEstrus cqui au moment où elle allongeạit singulierement son oviscapte pour pondre ses auls sur lépaul. du quadrupède. Cette femelle était par conséquent dans un état de gestation avancée, et je m'empressai de procéder à son autopsie.

Les ovaires se présentent chacun sous la forme d'un faisceau ovoïde ou ellipsoïdal, plus ou moins aplati à sa face inférieure, composé d'une qumtité innombrable (d'une centaine au moins) de gaînes ovigères allongées. Celles-ci m’ont paru uniloculaires; du moins je n'y ai trouvé qu'un seul œuf, malgré une gestation à terme. Le bout atténué de la gaîne, plus long que l'œuf, ne m’a offert auçune trace ni de locules, ni d'étranglements. Quand on cherche à constater le mode de connexion de ces gaînes entre elles on reconnait que le faisceau ovarien est rameux, c'est-ì-dire que les gaines s'insèrent par un pédicelle ou col à un axe tubuleux ou tronc central qui doit ètre considéré comme un culice. 
Le col de l'ovaire est court, mais bien marqué. L'oviducte, un peu plus long que lui, est cylindrö̈de. L'oviscapte est, comme dans beaucoup de Diptères, un étui à trois tuyaux engainés et rétractiles, qui, au moment de la ponte, se désemboitent et surpassent en longueur l'abdomen, mais il ne faut pas croire que cet instrument soit destiné à percer la peau du cheval comme le fait, d’après Réaumur, celui de l'Hypoderme. L'orstre pond ses œufs, que je décrirai bientôt, au milieu des poils du cheval.

La glande sébifique est placée, non à l'origine, mais à l'extrémité postérieure de l'oviducte, et m'a paru logée dans le premier tuyau de l'oviscapte. Je ne saurais disconvenir que je l'ai imparfaitement étudiéc. Il y a deux orbicelles ovalaires, brièvement pédicellés, à centre obscur. Les réservoirs séminaux sont deux bourses ovales oblongues, diaphanes, atténuées en col.

Les. cufs, d'un roux jaunâtre dans l'ovaire, deviennent d'un grisblanchâtre après avoir été pondus. Ils sont oblongs, conoïdes, sensiblement comprimés, obliquement tronqués au gros bout qui est antérieur dans la gaine, atténués en pointe par le bout opposé: la femelle les pond isolément lo long du poil du cheval, te plus souvent aux jambes. Ils sont intimement collés au poil, surtout par le gros bout. Celui-ci présente à sa troncature un opercule blanc, ovalaire, presque plane, destiné à se détacher en tout ou en partie, lors de l'éclosion de la larve, éclosion dont j'ai souvent été témoin. Ce fait très-positif confirme pleinement l'opinion du célèbre hippiatre anglais Clark, et réfute colle de Réaumur, d'après Gaspari, qui prétendait que l'œstre introduisait les œufs dans l'intestin du cheval par l'anus. Suivant Clark, c'est en léchant les endroits où les oufs avaient été déposés et oú les petites larves viennnent de naître que celles-ci sont entrainées d'abord dans la bouche, puis dans l'estomac du quadrupède ${ }^{3}$

2 Les ovaires, ainsi que l'appareil sébifique et séninal de l'OE. hamorrhoidalis, auraient d'après la description et les figures de M. Joly, une organisation parfaitement identique d̀ ceux de l'OE. cqui. Ceux de l'Hypoderma boris seraient aussi, selon ce même áuteur, formés sur le meme plan: 
Quclle singulière, quelle étonnante manourre! pour accomplir cette double et inconcevable destinée!... D'une part, obligation indéclinatoire du cheval d'introduire dans ses entrailles des vers qui doivent les ronger; de l'autre, mission innée d'une faible larve d'être avalée pour pouvoir vivre. Ne dirait-on pas qu'une mère dénaturée, ou pour le moins imprévoyante, a jeté ses enfants à une distance infranchissable du lieu ou doivent se trouver les conditions propres au maintien de leur vie et de leur prospérité? Né au milieu des poils de la jambe du cheval, c'est pourtant dans l'intérieur de l'estomac de celui-ci qu'est la subsistance de ce ver si délicat. .Il attend presque du hasard sá translation brusque et instantanée dans ce viscère. Vous allez voir comme le génie créateur a tout disposé pour le succès de ce rapt par un coup de langue. Je répète toujours avec un sentiment profond de conviction el d'admiration que, dans la structure si prodigieusement variée des insectes, il n'est pas une saillie, un poil, une ride qui n'aient une destination fonctionnelle, si l'on sait bien les interpréter. La larve de l'ostre a, dès sa naissance, plusieurs ceintures d'épines arquées et mobiles bien décrites et figurées par Réaumur. Sa bouche est armée de deux mandibules rétractiles en forme de harpons, et dans les efforts que fait ce ver pour sortir de son berceau j’ai constaté autour de son court promuscide une couronne de spinules crochues que n'a point signalée l'auteur précité. Eh bien! ces aspérités, ces harpons, servent d'abord à la jeune larve à exciter, titiller le tégument de son hòte, à y déterminer un prurit qui provoque à l'instant le lécher du cheval. Ces mêmes aspérités, ces mèmes harpons, changent aussitôt de rôle, et s'accrochent solidement à la langue dı ravisseur, pour l'abandonner quand celle-ci est rentrée dans la bouche. Ces unêmes spinules, ces mèmes crocs, sont encore pour la larve, parvenue dans l'estomac, des instruments de préhension, de trituration, de reptation et de fixation... Il faut s'humilier devant les grandes merveilles de ces petits êtres!

En ouvrant l'abdomen d'une femelle de Cephalemyia, je fus 11 . 
frappé de voir s'échapper par l'incision un nombre prodigieux de petites larves vivantes. Je les évaluai à quatre on cinq cents. Je reconnus bientôt que ces larves étaient renfermées dans deux grands sacs ovo-larvigères, analogues à ceux des Sarcophages, que je décrirai plus tard. Au-dessous de ces sacs, je trouvai les véritables ovaires vides sous la forme de deux faisceaux ronds munis d'un col.

Depuis Vallisnieri et Réaumur, on n’a pas cessé de répéter que l'œstre du mouton, ou Cephalcmyia, pondait ses cufs dans les fosses nasales de ce quadrupède. Il était réservé à l'anatomie de prouver que ce Diptère est réellement vivipare ${ }^{1 .}$

\section{FAMILLE DES MUSCIDES CALYPTEREES.}

J'avais d'abord voulu établir autant d'histoires anatomiques qu'il y a de sons-tribus dans les Muscides de M. Macquart. J'étais même fort avancé dans ma rédaction, lorsque je me suis convaincu que mon texte allait, contre mon intention, s'étendre, se dilater outre mesure. Le relevé statistique que je venais de faire de la composition du système nerveux et de l'appareil respiratoire dans toute la série des espéces disséquées, m'avait révélé un fait précieux pour la classification, c'est que, dans la vaste population des Muscides, il existe deux divisions bien tranchées. Dans l'une, il n'y a qu'un seul ganglion rachidien, et l'abdomen a toujours deux aérostats : elle embrasse les Créophiles et Anthomyzides de M. Macçuart, représentés par les Calyptérées de M. Kobineau-Desvoidy, dénomination plus convenable que j’ai adoptée. Dans l'autre division les aérostats abdominaux font défaut, et il y a plus d'un ganglion rachidien; elle comprend les Acalyptères

I M. Joly dit ce même Cephalemyia ovipare. Il se fonde sur ce quill a trouvé de véritables neufs dans les ovaires de cet OEstride; mais il ne repousse pas l'idée que ces oufs pourraient n'ètre tels qu'arant la fécondation: c'est lì une prudente circonspection. L'existence simultanée des sacs ovo-larvigères, que mon savant ami n'aura point vus, et des ovaires, confirme le fait de la viviparturition. Jajouterai, pour lever tous les doutes, que ces larves intra-utérines avaient les mêmes piquants qui les caractérisent hors du sein maternel. 
de M. Macquart. Ce sont lid deux grandes familles que plus tard on pourra partager encore.

Les Muscides calyptérées dont j’ai étudié l'anatomie sont les suivantes, en indiquant pour l'ordre de l'exposition de mes recherches les groupes qui correspondent aux sous-tribus de M. Macquart:

TACHINAIRES.

1. Echinomyia grossa. Dus.

2. - rubescens, R. D.

3. Gonia hebes. MEIG.

4. Siphona cinerea. Id.

5. Eurigaster antiqua. Mace.

6. Masicera sylutitica. Id.

7. Sericocera compressa. R. D. OCYPTÉRÉES.

8. Ocyptera bicolor. OL. GYMNOSOMÉES.

9. Gymnosoma rotundata. MEIG. PIIASIENNES.

10. Phasia crassipennis. Latr. DEXIAIRES.

11. Prosena sibirica. S. FARG.

12. Dexia rustica. Mace. SARCOPIIAGIENS.

13. Sarcophaga carnaria. MEIG.

14. hoemorrhoidalis. Id. WUSCIES.

15. Stomoxy's calcitrans. Geoffr.

16. Idia fasciata. MEIG.
17. Rlyyncomyia ruficeps. F.

18. Lucilia Caesar. Id

19. - violacea. MAcQ.

20. Calliphora vomitoria. R. D

21. Musca domestica. L.

22. Pollenia rudis. R: D.

23. - _ crerulescens?

24. Curtonevra maculata. MacQ.

25. … - pratorum. Id

26. horlorum. Id.

27. - - stabulans. Id.

ANTHOMYZIDES.

2x. Aricia lardaria. Mace.

29. - crratica. Id.

30), - - urbana. Id.

31. impunctala. Id

32. Spilogaster fossarum.

33. Hydrotrea dentipes. Maco.

34. Ophyra leucostoma Id.

35. Lispa teniuculata. LATr.

31. - - tarsalis. R. D.

37. Hylemyia strigosu. Mace.

38. - fuscata. MEIG.

39. Chortophila floralis. Mace.

40. Anthomyia radicum. Meig.

41. - caniculuris. F.

42. Pegomyiablepharipleroides. DuF.

Avant d'aborder l'anatomie de cette foule de Diptères, je vais faire connaitre quelques observations générales ou particulières qui les concernent. 
1. Les dissections m'ont appris que les Tachinaires, du moins les Echinomyia, Gonia et Siphonia, dont jai étudié l'appareil génital femelle, sont vivipares.

2. L'Echinomyia rubcscens, R. D. a été connu de liéaumur (t. IV, pl. 29, fig. 9), et c'est le Musca vivipara de Fourcroy (Ent. pur.).

Réaumur, qui a consacré un de ses beaux mémoires á l'histoire des mouches vivipares, signale, figure et décrit deux espèces. L'une pénètre dans nos maisons et dépose ses petits sur la viande: c'est la mouche grise vivipare, le Sarcophaga carnaria des modernes. Lautre, dit cet observateur, se trouve sur les fleurs: clle a la forme courte et renflée de la mouche bleue de la viande; la palette de ses antennes, au lieu d'être prismatique, comme dans la première, est lenticulaire, et la couleur du ventro est d'un brun feuille morte, avec une raic dorsale noire. Tout cela est conforme à l'Ech. rubescens. Mais Réaumur, séduit par le caractère vivipare de ces deux Diptères, tomba dans une erreur flagrante d'induclion, en regardant comme un trait commun aux deux espèces une matrice roulée en spirale qu'il n'avait constatée que dans l'Echinomyia. Il a donc commis une grave méprise, comme je le prouverai bientôt, en attribuant au Sarcophaga une semblable matrice.

3. Le genre Siphona, que Latreille, induit en erreur par la forme bicoudée de sa trompe, plaça dans les Conopsaires, et que Meigen, mal inspiré, a colloqué dans sa famille des Stomoxides, devrait peut-être se rapprocher des Dexiaires, dont il a la vivacité, le genre de vie, l'organisation viscérale et la viviparturition. Il n'irait pas mal à cause de sa trompe à côté du Proscna.

4. Le Phasia crassipennis, Meig. n'est pas un mâle, comme l'a avancé M. Macquart, mais bien une femelle, ainsi que le dit avec raison M. Robineau-Desvoidy. L'anatomie de l'organe génital a jugé en dernier ressort.

5. Les Dexiaires que j’ai disséquées sont évidemment vivipares. J'ai trouvé dans leurs matrices des petits vivants et en 
grande quantité. Ce fait confirme les observations de M. Pobineau-Desvoidy, sur la viviparturition de ses Macropodées, qui renferment le Dexic et le Prosena.

La larve du Dexia, que j’ai étudiée dans le sein de sa mére, est apode, céphalée, antennée, oblongue, glabre, de douze segments, la tête non comprise, atténuée en arrière, avec deux soies anales assez longues et deux appendices rétractiles, tronqués, noiràtres; jen donne la figure.

6. Les Muscies, dans la série des genres soumis à mon scalpel, présentent, dims leur appareil wribal mite, deux divisions fort

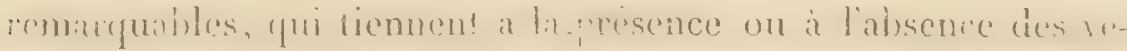
sicules scminales; mais, ce qu'il y a de plus remarquable encore, et ce qui fournit une nouvelle preuve de la concordance qui existe entre les viscères et les signes extérieurs, c'est que les genres Stomoxy's, Idia, Rhyncomyia, Lucilia et Calliphora, qui se suivent dans la méthode de M. Macquart, forment la division oủ les vésicules séminales existent, et les Ilusca, Pollenia, Curlonevra, celle où ces mêmes vésicules manquent.

7. Les Anthomyzides offrent la même division anatomique que les Muscies: Dans celles-ci, ce sont les derniers genres de la soustribu qui manqưent de vésicules séminales, tandis que, dans les Anthomyzides, ce sont les premiers. Ce signe négatif est, comme on voit, favorable dans ce cas à la série générique, mais non à la délimitation des sous-tribus; en sorte que, en ne consultant que ces organes, les genres pourvus de vésicules devraient appartenir à un groupe, et les autres à un autre. Je suis loin de prétendre qu'il doive en être définitivement ainsi; mais, à mesure que les faits de ce genre se multiplieront, ils pourront prêter leur concours à la classification. Quoi qu'il en puisse advenir, j'ai constaté l'absence de ces vésicules dans les genres Aricia, Iydrotea et Ophyra, et leur présence dans les genres Lispa, Hyicmyia et Anthomyia.

8. Les individus nombreux du Lispa, que je crois être le Tarsalis R.-D. (Myod. p. 526), et que j’ai disséqués, appartenaient tous 
¿ des mâles, et ceux du Tentaculata tous à des femelles. J'ai la conviction intime qu'ils ne sont que les deux sexes d'un même lype, d'une même espèce. Je les ai pris l'un et l'autre dans la même localité, dans le même instant. Ils n'habitent pas toujours les bords des rivières ou des flaques d'eau; je les ai souvert trouvés dans les allées de mon jardin, après un jour de pluie il esl vrai.

Les tarses antérieurs du Tarsalis sont tantôt entièrement roussâtres, tantôt avec le premier article noir et le dernicr obscur. Ces tarses sont un peu irréguliers à cause de l'insertion excentrique du second article sur le premicr; celui-ci est plus gros et du double plus court que le second; ce qui est contraire dans les autres pattes. Ce second article est comme flexueux. Les cuisses antéricures sont velues, légèrement cambrées; les autres sont glabres et droites.

\section{GHAPITRE PREMIER.}

APPAREIL DIGESTIF.

Los Muscides calyptérées fréquentent, pour la plupart, les lleurs, et s'y nourrissent, soit du pollen, soit des sucs mielleux; d'autres recherchent les substances mucilagineuses, sucrées, etc.

Les glandes salivaires ont en général une ténuité presque capillaire ct une longueur qui varic suivant les espèces. Dans l'Ech. grossa et le Gonia, elles sont beancoup plus longues que le corps, et se roulent, à l'issue de la tête, en un peloton compacte ovale ou oblong. Ce dernier est favorable à la stase, à l'élaboration de Ia salive; il tient lieu de réservoir. Dans les Dexia, Lucilia violacea, Pollenia, le vaisseau salivaire offre une agglomération lâche. Il atteint à peine le milieu de l'abdomen dans l'E. rubescens, le Sericocera, les Siphona, Ocyptera, Gymnosoma, Prosena, Stomoxys, Idia. Celui des Sarcophaga offre près de la tête une dilatation ellipsoïdale, un véritable réservoir salivaire. Dans le Lucilia Cresar, 
on trouve en même temps, et le réservoir de la sarcophage, et le peloton compacte de l'E. grossa. C'est là un perfectionnement organique digne de remarque. Enfin, ce vaisseau est fort long et très-flexueux dans les Rhyncomyia, Musca, Cartonevra, Calliphora, IIylemyia, etc.

Le canal alimentaire a, en général, une longueur qui surpasse de trois, de quatre et même de cinq fois celle de l'insecte, de deux fois seulement dans les Eurigaster, Masicera, Dexia, Prosenu. La panse a un réservoir bilobé. Le ventricule chylifique débute. dans tous, par un godet orbiculaire ombiliqué : ce trait ne se dément jamais; puis il se continue en un conduit cylindroïde ou filiforme d'une même venue partout, tantôt enroulé en une, deux ou trois circonvolutions, tantôt se bornant, comme dans les Eurigaster, Masicera, à une seule anse ou à de simples flexuosités dans les Dexia, Prosena.

Les vaisseaux hépatiques, en général jaunes, longs, plus ou moins variqueux, parfois subgranuleux, s'unissent par paires, it deux canaux cholédoques bien marqués. Ceux-ci sont, exceptionnellement dans l'Ocyplera, d'une telle brièveté, qu'ils paraissent nuls. Ces vaisseaux, dans les Gonia, Siphona, Eurigaster, Masicera, Sericocera, Prosena, sont bien plus courts et plus gros.

L'intestin, d'abord grêle et filiforme, se renfle en un rectum assez gros, ayant ordinairement, à sa moitié antérieure, quatre boutons charnus orbiculaires ou pyramidaux. Je n'ai trouvé que deux de ces boutons dans l'Eurigaster. Il n'en existe pas dans les Dexia et Prosena. Ce caractère négatif n'est pas sans valeur.

\section{CHAPITIE II.}

APPAREIT, GENITAT.

ARTICLE ${ }^{\text {er }}$

APPAREIL GENITAL MALE.

Les testicules du plus grand nombre de ces Muscides sont d'un brun marron ou chocolat; les Echinomyia, Gonia, Euriguster, 


\section{RECHERGHES ANA'TOMIOUES LT PHYSIOLOGIQUES}

sont les seuls genres oú jai trouvé ces organes blancs, ou d'un jaune très-pâle. Les Tachinaires les ont, en général, sphéroïdes, terminés par une sorte de bec droit, qui ne semble que la tunique testiculaire vide et contractée. J'ai même vu, dans quelques individus du Siphona, ou ces organes étaient sans doute fort turgescents, ce bec entièrement effacé. Il n'existe pas dans le Sericocera. Le testicule de l'Eurigaster est tantôt étranglé dans le milieu comme une calebasse, tantôt oblong; allongé, parfois fusiforme dans les Ocyptera, Sarcophaga, Rhyncomyia; oblong dans les Aricia, Lispa; ovoïde ou puriforme dans le Gymnosoma, les Muscies et le plus grand nombre des Anthomyzides. Les Muscies, pourvues de vésicules séminales, comme les Stomoxys, Idia, Rhyncomyia, Lucilia, Calliphora, ont une texture tont à fait exceptionnelle de cet organe; il a, indépendamment de sa tunique propre colorée, une enveloppe extérieure accessoire, incolore, d'un tissu charuu plus ou moins condensé.

Les conduits déférents sont capillaires; un peu plus longs que tes testicules dans la plupart des Tachinaires, dans les Ocyptera, Gymnosoma, Lucilia surtout, Calliphora; bien plus courts et bruns dans les Siphona et Lispa; aussi courts mais incolores dans les Idia, Rhyncomyia, Surcophaga, Musca, Curtonevra, Ariciu et les autres anthomyzides; prescrue nuls dans le Proscna.

L.es vésiculessiminales, quand elles existent, sont toujours simples pour chaque côté. Elles recoivent les conduits déférents immédiatement avant leur confluence pour la formation du canal éjacula1eur. Elles sont ovales ou elliptiques dans l'Ech. grossa, les Eurigaster, Sericocera, Gymnosoma, Rhyncomyia, Iylemyia Strigosa; allongées, filiformes, plus ou moins repliées dans l'Ech. rubescens, les Sarcophaya et Lispa; longues et presque capillaires dans l'Ocyptera; en massue et courbées en crosse dans le Prosena; simplement oblongues dans les Idia, Lucilia, Calliphora. Rappelons-nous qu'une division des Muscides et des Anthomyzides, ainsi que je l'ai dit, manque de vésicules séminales; ce sont les Musca, Pollenia, Curlonevra, Aricia, Hydroten, Ophïr, et partie des Hylemyia. 
Le canal ćjaculateur est filiforme et médiocrement long dans les Tachinaires, les Ocyptera, Gymnosoma, Idia, Rhyncomyia, Lispa, IIylemyia strigosa, Anthomyia; bulbeux à son origine dans les Gonia, Eurigaster, Sarcophaga. Ce bulbe est oblong dans les Lucilia, Calliphora. Dans les Muscides calyptérées, privées de vésicules séminales, le canal éjaculateur reçoit directement à son origine les conduits déférents, et il se fait remarquer par ses flexuosités et sa longueur. Celle-ci égale sept à huit fois celle du testicule. Ces deux conditions de la longueur et des replis suppléent à l'absence des vésicules en fournissant au sperme récemment secrété l'occasion de s'élaborer par son séjour et par ses balancements organiques.

Je ne m'explique pas l'énorme différence qui existe entre les appareils génitaux mâles de l'Hylemyia fuscata et de l'H. strigosa. Dans cette dernière espèce, il existe deux vésicules séminales ovoïdes et un canal éjaculateur de longueur médiocre, tandis que l'H. fuscata rentre dans la série des Muscides privées de ces vésicules et pourvues d'un canal éjaculateur fort long et très-flexueux. Cette longueur dans l'H. fuscata égale deux fois celle de tout son corps. Cet appareil ressemble trait pour trait à celui du Curtonevra maculata. Une si grande dissemblance organique porterait à croịre que nos deux Hylemyia n'appartiennent pas à un même genre, et cependant, en étudiant comparativement leurs traits extérieurs, on ne saurait se refuser à leur identité générique. Dans mes dissections de l'H. strigosu, qui datent de sept à huit ans, aurais-je commis quelque méprise? Je le crains.

L'armure copulatrice, organe des distinctions spécifiques, est, dans toutes ces Muscides, logée, enchatonnée sous le bout de l'abdomen. Lorsqu'elle entre en exercice, elle peut se débander comme un ressort. On la met facilement en évidence par une compression expulsive de l'abdomen. Son extrémité, qui regarde en arrière, s'engage plus ou moins, dans le repos, entre ou sous des pièces particulières qui dépendent du dernier segment ventral, et qui servent à la relenir. Pour compléter mon travail sur ce point, je devrais décrire toutes les armures des Muscides disséquées. Une

11 . 
semblable monographie m'entraînerait beaucoup trop loin; et je me bornerai a l'exposition succincte de quelques types.

L'armure de l'Ech. rubcscens étudiée par sa face externe se compose : $1^{\circ}$ d'une pièce basilaire subannulaire coriacée; $2^{\circ}$ d'un forceps à branches oblongues noirâtres, velues, tronquées au bout, mais articulées avant avec une pièce triangulaire velue, faisant l'office de crochet; $3^{\circ}$ d'un fourreau de la verge allongé, cornéomembraneux, avec quelques aspérités, terminé par deux pointes brunes à peine courbées.

Celle du Sericocera a une singulière complication. Le forceps a ses branches grosses, velues, très-écartées, terminécs en pointe simple et droite. I.a volselle, enchassée à la base du forceps, est aussi longue que lui, et composée de cleux lames adossées. Elle se dilate à sa base, qui est velue et échancrée en demi-lune, puis elle est effilée et droite avec sa pointe bifide. Le fourrean de la verge a une configuration insolite, qui doit faire supposer de bien curieuses mancuvres dans l'union des sexes. Il est rétréci à son origine, qui est flanquée d'une sorte de balancier formé d'une tige subsinueuse, poilue à son extrémité. Puis il est ventru, ellipsoïdal, et se termine par deux crochets pointus divergents, inclinés sur les côtés, et entre lesquels est l'orifice qui donne issue au pénis.

Sa composition dans l'Ocyptera est anormale et il n'est pas facile d'adapter aux diverses pièces constitutives la nomenclature ordinaire. Dans son ensemble, elle est arrondie. Le forceps, qui forme à l'extérieur un demi-cintre, est un arc de cercle très-ouvert. Ses branches noires et velues sont obtuses à leur extrémité. Entre ces branches est enclavée une grande pièce arrondic, modérément convexe, velue, de couleur ambrée, terminée brusquement en arrière par un petit bec droit refendu au bout. Cette piéce semble l'analogue de celle que j’ai appelée volsclle dans le Sericocera mais elle n'est pas composée de deux moitiés scmblables, circonstance qui me la ferait plutôt regarder, sinon comme un fourreau, du moins comme un réceptacle de la verge. Entre elle et le forceps, on voit de chaque côté une plaque blanchâtre, glabre, cornée, terminée 
par un bout particulier noir et velu. Mais outre cela il y a en dessous des pointes, des lames, des crochets, dont je n'ai encore saisi ni les connexions, ni les attributions.

L'armure du Sarc. homorhoidalis, envisagée dans son ensemble, forme un corps arrondi. La pièce basilaire coriacée, convexe, velue et d'un roux fauve luisant, forme le bout de l'abdomen. Elle offre en arrière une profonde échancrure à angle aigu ou en $\mathrm{V}$, au fond de laquelle s'ouvre l'anus. Le forceps est noir, à deux branches mandibuliformes glabres, presque droites, à pointe aigue, mais fort courte. De la base du forceps partent deux tiges courtes, oblongues velues, de même couleur et de même consistance que lui, faiblement arquées, adossées ensemble par leur convexité et enclavées dans l'échancrure de la pièce basilaire. Ces deux tiges appartiennent peut-être à une volselle incomplète. Au centre de l'apparcil, est le fourreau de la verge, du moins je ne saurais lui donner une autre dénomination. Il est oblong, corné, noirâtre, glabre, tronqué au bout, avec une ligne médiane enfoncée qui semble indiquer deux moitiés semblables. Les côtés ont, au milien, une légère saillie dentiforme. Une forte compression expulsive exercée sur le fourreau détermine la saillie de deux lames subtriangulaires qui m'ont paru être les panneaux de l'ouverture par oú doit sortir le pénis. Au-dessous du bout tronqué on découvre de chaque côté un crochet glabre, très-courbe, terminé par une spatule arrondie, oủ le microscope décéle de fines aspérités marginales. A la racine inférieure ou, si l'on veut, interne du fourreau, il y a à droite et à gauche une paire de crochets arqués et pointus dont le plus postérieur est plus fort que l'autre. Les pointes du forceps se trouvent, dans la condition du repos, en regard de deux tiges cornées, velues dans une portion de leur bord interne et faisant partie de la dernière plaque ventrale. Comme on te voit, cette armure a une curieuse constitution. Nous sommes encore bien loin d'être fixés sur le rôle respectif de tous ses ressorts.

Parmi les Muscies, l'Idia a une armure petite, ovalaire, brune, glabre, armée en dessous de quatre stylets noirs, presque droits, 
dont deux appartiennent sans doute au forceps et les deux autres au fourreau. Le dernier segment ventral a deux crochets arqués qui combinent leur action avec les stylets.

Cette armure est aussi ovalaire dans le $L$. Cresar. La pièce basilaire noire, de texture tégumentaire, hérissée de longs poils, présente en arrière une grande échancrure à angle aigu, oủ s'ouvre l'anus. Le forceps a ses branches brunes, glabres, fines, pointues, un peu courbées à leur extrémité, logées entre deux panneaux oblongs et inermes du dernier segment ventral. Le fourreau est formé par l'adossement de deux lames étroites, brunes, pointues.

Celle du Calliphora est bien plus allongée que la précédente, et son extrémité est dans une grande échancrure voûtée du dernier segment du ventre; la pièce basilaire est presque carrée. Les branches du forceps, hérissées de quelques poils, sont arquées et terminées par un bout en cueilleron. Le fourreau, aussi long que le forceps, est formé de deux tiges presque droites.

L'armure du Curt. maculata est fort petite. La pièce basilaire est un bourrelet transversal un peu arqué, velu, coriacé. Les branches du forceps, courtes velues et subtriangulaires, se terminent par un cueilleron d'un brun luisant séparé du corps de la branche par un petit étranglement où il y a une articulation linéaire favorable à ses mouvements. Deux lames lancéolées conniventes formeraient le fourreau.

Parmi les Anthomyzides, le Lispa a l'armure ovalaire. La pièce basilaire est un bourrelet noir, coriacé, velu, courbé en demicercle et obtus à ses extrémités, avec une articulation linéaire au milieu. Le forceps est formé de deux larges panneaux coriacés, blonds, velus, contigus, échancrés au bout avec l'angle interne prolongé en pointe. De dessous l'échancrure, sort un stylet grêle, noir, pointu, comme flexueux, qui appartient peut-être à une volselle. Le dernier segment ventral offre ici une plaque trifide où se logent les stylets.

Celle de l'Hylemyia strigosa ressemble à la précédente par la forme de sa pièce basilaire, mais les branches du forceps sont 
légèrement courbées pour former'la pince. Les pointes de celle-ci s'engagent, dans le repos, entre deux placques ventrales oblongues hordées extérieurement de longues soies.

ARTICLF II.

APPAFEIL GÉNITAL FEMELLI.

Cet appareil va nous offrir dans les Muscides calyptérées úne configuration, une composition qui ne se sont pas présentées dans les familles précédentes. Jusqu'ici, si nous en exceptons quelques OEstrides (qui sont aussi calyptérées), les œufs, après avoir acquis dans les gaines ovigères toul leur développement, descendaient dans le calice pour y compléter leur maturité, et étaient pondus au-dehors en passant par l'oviducte. Nous allons trouver maintenant des genres vivipares et d'autres ovipares, par conséquent des organes femelles de la génération fort dissemblables.

Dans les Tachinaires, les œufs à terme sont déposés dans un organe particulier placé à la suite de l'oviducte, où ils sont destinés à éclore avant d'avoir vu le jour; en sorte que les femelles sont alors vivipares. D'après ces attributions physiologiques, j’ai désigné cet organe sous le nom de réservoir ovo-larvigère. Ce serait une matrice. Décrivons l'appareil dans l'Ech. grossa.

Les ovaires sont deux rondelles ou plateaux déprimés, garnis de rangées circulaires de gaînes ovigères, multiloculaires, innombrables, dont les pointes effilées convergent au centre du plateau. Les séries extérieures sont les premières fécondées et les plus grosses; aussi forment-elles une sorte de bourrelet. Ces réceptacles de la progéniture sont maintenus en place par de nombreuses rênes trachéennes fournies à chacun d'eux par deux ou trois troncs de ces vaisseaux aérifères. Les cols des ovaires sont courts,' grêles, mais renflés à leur origine. Ils confluent en arrière pour la formation de l'oviducte, qui est plus long qu'eux, mais uniformément grêle. Le réservoir ovo-larvigère suit immédiatement et directement l'oviducte. C'est un long boyau déprimé, roulé sur 


\section{RECHERCHES ANATOMIQUES ET PHYSIOLOGIQUES}

lui-même, de manière à former trois tours de spire, et tout couvert à l'extérieur de points en relief arrondis, régulièrement disposés, soit en long, soil en travers. Il renfermait, dans les individus dont jai fait l'autopsie, une quantité prodigieuse, des milliers d'œufs à terme, petits, grêles, allongés, étroitement pressés les uns contre les autres, mais non entassés, fixés par un bout aux parois du boyau. Ces bouts, par leur légère saillie au dehors, forment ces points ronds dont je viens de parler. Si l'on déchire ce réservoir pour étudier le mode d'insertion des cufs, on voit, dans les lambeaux, qu'ils y adhèrent par un seul bout, de manière à paraître pendants, absolument comme les papilles qui revêtent le dessous du chapeau des champignons du genre $\mathbf{H y} d-$ num. Il résulte de cette disposition des oufs, que leurs bouts libres correspondent à l'axe fictif du boyau. Le réservoil ovo-larvigère se termine à la vulve, et avant la fécondation il forme un conduit qui peut être considéré comme un vagin.

Le phénomène organique de la fixation des ceufs par un seul bout et par séries contre les parois internes de ce réservoir tubuleur. est sans doute en partie sous la dépendance de la glande sébifique, que j'examinerai bientôt, mais il tient aussi à des affinités vitales intimes, à des lois' secrètes d'organisme qu'il n'est pas encore permis de formuler.

Je n'ai pas eu occasion de constater la présence des larves dans le réservoir ovo-larvigère de l'Ech. grossa, parce que sans doute les femelles que j’ai disséquées n'étaient pas assez avancées dans la gestation. Mais dans l'Ech., rubescens, où il existe un semblable réservoir avecles mêmes connexions, ¡’ai rencontré, en même temps, et des larves vivantes et d'autres à demi écloses, et des oeufs enliers et des coques d'œufs vides. J'ai trouvé ce réservoir farci de larves dans le Siphona et le Gonia. Enfin, je n'hésite pas à répéter que toutes les Tachinaires sont vivipares, et ce fait nouveau est acquis à la science par les études anatomiques.

Dans le Gonia, le réservoir ovo-larvigère est plus long que celui des Echinomyia, filiforme et très-reployé. Les oufs à terme 
sont ovales, pointus par un bout et d'un noir profond. Les ovaires du Siphona, au lieu d'être en rondelles, comme dans les genres précédents ont leurs gaînes ovigères, disposées en un faisceau oblong, et le réservoir ovo-larvigère est moins long que dans les Échinomyies.

Passons à l'appareil sébifique el séminal. Puisque les Tachinaires sont vivipares, qu'était-il besoin d'une glande destinée à enduire les œufs d'un vernis préservatif ou conservateur? Et cependant celte glande existe avec toutes les conditions d'un organe sécréteur comme dans les Diptères ovipares. Mais l'anatomie vient de nous apprendre que si les Tachinaires ne pondent pas des œufs au dehors, elles les pondent au dedans du corps, et que ces oufs, pour se rendre au réservoir ovo-larvigère, où ils doivent subir une incubation jusqu'à la naissance des larves, ont nécessairement à passer sous l'orifice excréteur de cette glande, pour y recevoir l'ablution sébacée? N'avons-nous pas vu encore qu'indépendemment d'un séjour dont nous ignorons la durée, les oufs sont collés par un de leurs bouts aux parois du réservoir ovo-larvigère. Ces circonstances, bien comprises, ne sont-elles pas faites pour confirmer notre opinion sur les fonctions sébiliques de cette glande?

Dans l'E. grossa la glande sébifique a pour organe sécréteur trois orbicelles ovalaires à centre largement noir, contigus et sessiles, sur un col excréteur commun d'une excessive brièveté. Les réservoirs séminaux sont deux hourses en massue allongée, insérées à droite et à gauche du col précédent, insertion fort remarquable, parce qu'elle ne se fait pas immédiatement sur l'oviducte. Dans $l^{\prime} E$. rubescens les orbicelles sont arrondis, munis de cols efférents bien marqués. Ceux du Gonia, plus grands et ovales, avec du gris au centre, sont longuement pédicellés, avec des réservoirs séminaux, filiformes et flexueux. Le Siphona ressemble par l'appareil sébifique et séminal plutôt aux Dexiaires, auxquelles j’ai déjà dii qu'il pourrait se rallier, qu'aux T'achinaires. Les orbicelles, au nombre de deux seulement, sont sphéroïdes et entièrement noirs comme dans le Prosena et le Dexia. On les croirait sessiles sur l'ovi- 
ducte, mais ils n'y sont qu'adhérents, on peut les en détacher, et mettre en évidence leurs longs cols capillaires, d'abord courbés en anse. Les réservoirs sont ovoïdes, avec un col plus long qu'eux.

Il est bien évident, d'après la forme et la composition des organes génitaux femelles des Gymnosoma, que ces Diptères ne sont pas vivipares. Mes dissections me permettent même d'annoncer qu'ils ne doivent pas pondre un grand nombre d'oufs. Les ovaires sont arrondis ou turbinés, formés chacun d'une douzaine environ de gaînes ovigères quadri ou quinqueloculaires. Le calice est peu apparent; le col, tubuleux, de la longueur de l'ovaire; l'oviducte, d'abord filiforme, puis dilaté en un réservoir ovigère qui remplace le calice, et que nous retrouverons désormais mieux conditionné. L'appareil sébifique et séminal a trois orbicelles subglobuleux, rapprochés, grisâtres, avec le centre plus blanc, ce qui est l'opposé de presque tous les autres, munis d'un col capillaire de peu de tongueur. Les réservoirs sćminaux sont oblongs, avec un col très-fin et court. Les $\propto u f s$ à terme ont une configuration singulière. Ils sont hémisphériques, plats en dessous, roux, avec un bourrelet circulaire blanc.

L'ovaire du Phasia présente avec le précédent plusieurs traits de ressemblance, qui prouvent leur affinité générique. Il n'a que sept à huit gaînes ovigères et celles-ci ne sont que biloculaires, en sorte que le Phasia pond encore moins d'oufs que le Gymnosoma, ce qui nous explique la rareté des Phasiennes. L'oviducte est moins long et se termine par un réservoir ovigère. La glande sébifique a la même composition, mais les orbicelles sont bruns au centre. Les œufs sont en segment de sphère, mais sans bourrelet.

J'ai acquis la certitude, par l'étude de l'appareil génital femelle dans ses divers états de gestation, que les Dexiaires, ou du moins les genres soumis à mon scalpel, sont vivipares. Dans la méthode naturelle, il conviendrait de les placer à la suite des Tachinaires. La conformité anatomique de cet appareil dans le Dexia et le Prosena légitime pleinement le rapprochement de ces deux genres, malgré l'énorme différence respective de leurs trompes. Dans 
l'individu du Dexia, dont j’ai dessiné les ovaires, ceux-ci étaient vides, tandis que le réservoir ovo-larvigère était plein d'œufs et de larves. Cette femelle touchait donc au terme de sa délivrance complète. Ces ovaires étaient ovalaires ou conoïdes, composés d'une vingtaine environ de gaines ovigc̀res multiloculaires. Le col "st court, l'oriducte tulunleux, cylindrique, suivi d'un réservoir ornlarvigère, qui n'est ni comprimé, ni roulé en spirale comme celui des Echinomyes, mais allongé cylindroïde ou fusiforme, flexueu. ou simplement courbé en anse. Les cuufs sont oblongs ou allongés blancs.

L'appareil sébifique ou séminat inséré à la terminaison de l'oviducte a trois orbicelles ovalaires entièrement bruns, contigus sur une même série et sessiles en apparence. Quand on cherche à constater leur mode de connexion, on s'assure qu'ils ont un col efférent plus délié qu'un cheveu et reployé sous l'orbicelle. Les réservoirs séminaux sont deux bourses en massue, atténuées en un long col qui s'insère à côté des conduits efférents précédents. Leur bout adhère fortement aux cols des ovaires, circonstance qui rend très-difficile leur dissection.

L.es ovaires du P'rosena, que j’ai surtout étudiés à cette période de la gestation où ils étaient compléiement garnis d'œufs, sont ovoïdes, composés d'un nombre indéterminable de gaînes ovigères multiloculaires. Le col et l'oviducte sont encore plus courts que dans le Dexia. Les oufs et le réservoir ovo-larvigère ressemblent en tous points à ceux de ce dernier genre. Les tentacules vulvaires sont velus, ovalaires, d'un seul article. L'appareil sébifique et séminal se compose des mêmes parties que dans le Dexia.

Quand l'observation directe ne nous aurait pas appris que les Sarcophagiens sont vivipares, l'existence d'un vaste réservoir ovolarvigère nous en eût donné la certitude; mais ce réservoir a ici une configuration bien différente de celle des fimilles précédentes. et elle constitue leur trait anatomique le plus distinctif. Ce n'est pas un réservoir unique plus ou moins allongé ou enroulé, mais un bissac, une double matrice. 
Les ovaires, placés au-dessous du bissac dont je viens de parter, consistrnt chacun en un faiscean arromeli, on conoide, de gaines ovigères innombrables, multiloculaires, tcrminćes par un fin ligament suspenseur. Leur col est tubuleux, presque de leur longueur. L'oviducte, plus long que les cols, s'abouche brusquement au canal éducateur du bissac ovo-larvigère. Les $œ u f s$, arrondis dans l'ovaire, deviennent oblongs quand ils sont à terme. Le bissac ovo-larvigère, lorsqu'il est bien rempli par les produits de la fécondation, se présente sous la forme de deux énormes bourses arrondies, contiguës, dont l'enveloppe est une fine membrane pellucide, qui permet de reconnaître dans son intérieur les œufs ou les larves. J'y ai compté à la fois plus de deux cents de ces dernières; mais la configuration de ces bourses varie suivant leur degré de réplétion. J'ai cru parfois qu'il n'y cxistait qu'un seul réservoir plus ou moins échancré en arrière. Chacun des sacs a un col fort court, qui s'unit bientôt à son congénère pour former le conduit édacatcur, qui, après avoir reçu l'oviducte, va s'ouvrir à la valve.

L'appareil sébifique et séminal ressemble, trait pour trait, à celui des Dexiaires; il s'insère à l'oviducte, immédiatement avant l'embouchure de ce dernier au conduit éducateur du bissac ovolarvigère. L'organe sécréteur se compose de trois orbicelles ovoïdes à centre brun, paraissant sessiles, mais munis d'un col fusiforme plus long qu'eux; les réservoirs sont deux bourses ovalaires atténuées en un col de leur longueur.

L'absence d'un réservoir ovo-larvigère dans les Muscies nous donne la certitude que ces Diplères ne sauraient être qu'ovipares. Je prendrai, pour type de la description de l'appareil génital femelle de ce groupe, celui du Lacilia Cacsar.

Les ovaires consistent en deux plateaux orbiculaires, qui, dans un étal avancé de gestation, occupent presque toute la cavité abdominale. Les gaínes ovigćres, innombrables et bi ou triloculaires, y sont rangées par séries circulaires pressées et concentriques. Comme la série extérieure est la seulc qui paraisse sur la tranche 
du plateau et cqu'elle est la plus développée à l'époque de la gestation, le disque de la rondelle, légèrement déprimé, apparail couvert de points ronds qui ne sont que les bouts saillants des œufs. Le col de l'ovaire est court, tubuleux, latćral; l'oviducte esl aussi tubuleux, mais plus long; les aufs à terme sont allongés.

L'apparcil sébifrque et séminal, placé à l'extrémité postérieure de l'oviducte, a trois orbicelles à centre noirr, à peu près sessiles dans le Calliphora, munis, dans les autres genres, d'un long col efférent capillaire. Les réservoirs séminaux ressemblent à la plupart de ceux des familles précédentes; ce sont deux boyaux simples dont les bouts renflés adhèrent aux cols des ovaires, tandis que, du côté opposé, ils s'étrécissent pour s'implanter conjointement avec les cols efférents. Ceux du Lucilia Cresar ont un petit col capillaire; ils sont tout à fait filiformes dans le L. violucea.

A en juger par la forme et la composition des ovaires, le genre lilyncomyziu ne derrait pas rester dans le groupe des Muscien. quoique, sans doute, il s'y rallie par des traits cxtérieurs; ses ovaires sont ovales-oblongs, de dix à douze gaînes ovigères seulement, uniloculaires. Le col est court, non latéral, et l'oviducte assez long. Celui-ci offre, vers sa terminaison, un corps particulier, arrondi et épais, un réscrvoir ovigère; les œufs sont ovales, obtus, blancs. L'appareil sébifique et séminal, comparé à celui des antres Muscies, ne présente pas la mème rlissemblance que les ovaires; il a au contraire avec elles la plus grande analogie de composition. Il y a, pour la sécrétion, trois orbicelles ovalaires, noirs, dont la tunique, d'une certaine épaisseur, est diaphane, et qui ont des cols capillaires assez longs, doublement fléchis à leur origine. Les réservoirs séminaux sont en massue allongée et adhèrent par leur bout au col des ovaires, comme à l'ordinaire. Par une compression expulsive, on voit saillir, au bout de l'abdomen, une pièce ovale triangulaire bordée de chaque côté par quatre ou cinq soies noires arquées. Cette pièce fait supposer quelque manœuvre particulière lors de la ponte des oufs. 


\section{RECHERCHES ANATOMIQUES ET PHYSIOLOGIQUES}

L'appareil génital femelle des Anthomyzides n'offre pas moins de différence que l'organe mâle dans les divers genres de ce groupe, et, sous ce rapport anatomique, la série de ceux-ci aurait quelques mutations à supporter.

Les ovaires de l'Aricia urbana, loin d'être en plateaux arrondis comme dans beaucoup de Muscies, sont en faisceau ovalaire ou oblong, assez lâche, de douze gaînes ovigères, allongées et triloculaires. Le calice est postérieur; le col fort court. L'oviducte, d'abord grêle, grossit ensuite pour devenir réservoir ovigère. L'appareil sébifique et séminal se compose de trois orbicelles ovalaires noirs, subsessiles, et de trois réservoirs en petits boyaux oblongs.

On trouve une tout autre conformation d'ovaires dans l'Hydrotea, insecte qui ressemble tant ì la mouche domestique. Ils sont en plateau orbiculaire comme dans celle-ci.

Ceux de l'Ophyra ont des gaines ovigères disposées comme dans le $L$. Cacesar; mais le plateau, au lieu d'être rond, est ovale. Le col est postérieur et court; l'oviducte, plus long, et les œufs, blancs et oblongs. L'appareil sébifique et séminal a pour la sécrétion trois orbicelles noirs, ovales, sessiles. Les réservoirs sont deux boyaux allongés, adhérents par un bout au col des ovaires, comme dans les Muscies.

Le Lispa, insecte de mœurs si particulières, va nous offrir dans ces organes des traits curieux de structure. Ses ovaires, loin de ressembler à ceux des Hydrotca et Ophyra, ont la plus grande analogie avec ceux de l'Aricia. Chacun d'eux est un faisceau ovalaire de quinze à vingt gaînes ovigères, allongées, multiloculaires. Le calice et le col ne différent pas de ceux de ce dernier genre; mais l'oviducte est long, filiforme et aboutit en arrière à un réservoir ovigèrc oblong, à parois comme calleuses.

Les aufs de la lispe ont une structure dont je n'ai pas vu d'autre exemple dans les Diptères. Ils sont grands, vu la taille de l'insecte, oblongs, plus ou moins cambrés, d'une teinte roussâtre. Ils ont une face convexe, finement striée, suivant sa longueur, et une face plane ou déprimée, parcourue par deux nervures sub- 
marginales, parallèles, terminées au bout antérieur par une spatule arrondie, débordant ce bout, élégamment bordée de longues soies arquées, au bout postérieur, par deux petites pointes sursaillantes subulées. Quel investigateur des habitudes de ce Diptère ripicole sera assez heureux pour nous apprendre le pourquoi de cette singulière structure!

L'appareil sébifique et séminal a aussi son originalité. Les trois orbicelles à centre noir, qui constituent son organe sécréteur, sont contigus en triangle et munis de lòngs cols capillaires. Ces trois cols s'unissent en un seul conduit qui s'insère à ła partie postérieure et dorsale du réservoir ovigère. Deux bourses ovalaires forment les réservoirs séminaux. Elles ont un col capillaire assez long, et les deux cols se confondent aussi en un seul conduit excréteur qui s'implante à côté de l'organe sécréteur.

L'Anthomyia se ratlache par la forme et 'a composition des ovaires et de la glande sébifique, à l'Hydrotea et au Musca.

FAMILLE DES MUSCIDES ACALYPTÉRÉES.

J'ai déjà dit que l'étude du système nerveux et des trachées m'avait déterminé à diviser l'immense nation des Muscides en deux grandes familles, au moins provisoirement. J'ai exposé mes recherches sur les Muscides calyptérées. Je vais maintenant entreprendre un semblable travail, mais pius difficultueux encore, sur les Muscides qui sont privées de cueillerons aux balanciers, d'où leur dénomination d'acalyptérées, en opposition avec celle de la famille précédente. L'absence des cueillerons s'accompagne de la privation des aérostats à l'abdomen. C'est là un fait qui a quelque portée. Mais les acalyptérées ont, indépendamment du cerveau, deux ou trois ganglions rachidiens, tandis que les calyptérées, qui ont une prééminence organique bien établie, n'en possèdent qu'un seul. C'est un autre fait qui pourrait soulever des questions intéressantes sur les rapports de la puissance nerveuse avec le nombre 
et le volume des ganglions. Il y a encore beaucoup à étudier et à méditer sur ce point.

Les diptérologistes, avec le seul secours des caractères extérieurs; se sont tous entendus pour considérer ces petites et faibles Muscides comme moins élevées que les autres en organisation, et ils en ont relégué les nombreuses peuplades à la fin de leur cadre générique. Eh bien, les dissections confirment cette infériorité organique et mettent le sceau à leur jugement. Ce résultat, en même temps qu'il témoigne hautement de leur tact, de leur habileté à apprécier la valeur des formes extérieures, est aussi l'hommage'le plus éclatant que l'on puisse rendre à la certitude de la science; il est une preuve authentique et consolante de cette admirable corrélation des actes de la vie avec les ressorts ou apparents ou secrets qui y président. Combien je m'estime heureux d'avoir mis au jour quelques-uns de ces derniers et d'en devenir l'interprète! Voyez comme l'absence d'un organe, auquel on avait d'abord accordé peu d'importance, a d'intéressantes conséquences dans l'organisme! La privation des cueillerons coincide dans nos Muscides, ainsi que je me plais à le répéter, avec la privation des ballons abdominaux; et ces deux traits négatifs entraînent, pour résultat physiologique sur l'ensemble de l'économie animale, ces habitudes paisibles et sédentaires, celle abnégation du grand jour, celte lente ambulation, ce sautillement hébété, ce vol faible et muet, ces teintes sombres, ce tempérament mélancolique, qui caractérisent si bien nos petites mouches acalyptérées.

Voici le catalogue de celles de ces dernières que jai soumises à mes vivisections, en conservant leur disposition par groupes ou sons-tribus.

DOLICHOCÈRES.

1. Sepedon sphegeus. FALL.

2. IIaeffnerii. Id.

3. Tetanocera marginata. MIEIG.

1. — sticlica. LATR.

5. reticulata. Id.
6. Tetanocera pralorum. FALL.

7. - ferruginea. Id.

8. — araloria. MEIG.

LOAOCÉRIDES.

9. Loxocera ichneumonea. Mivig. 
CORDYLURIDES.

10. Chytiza leptogaster. Fald.

11. Cordylura pubera. Id.

SCATOMZIDES.

12. Scatophaga stercoraria. MEIG.

13. Intaria. Id.

1'1. Sapromyza ustulata. Id.

15. Sapromyza rorida. Id.

16. Helomyza tigrina. FALL.

17. rufu. MAce.

18. pullirk. Id

ORTALIDÉES.

1). Orlatis vibrans. Fall.

21). - - lugens. MeIG.

21. Platystoma umbrarum. Id.

TÉPIIRITIDES.

22. Tephritis fasciata. Maco.

$$
\text { SEPSIDÉES. }
$$

2i. Sepsis punctum. FALL.

24. Cheligaster putris. MACQ.

2). Nemopoda cylindrica. Id.

LEPTOPODITES.

2i. Calobuta cothurnata. MEIG.

27. Micropeza thoracica. Mace.

$$
\text { ULIDIENS. }
$$

28. Ulidia demandala. Meta.
LAUXINIDES.

29. Lauxania eneu. F.ILI.

30. Lonchara larsala. Id.

HYDROMYZIDES.

31. Ochtera mantis. Litr.

32. Notiphila cinerea. FALL.

PIOPHilides.

33. Teichomy a fusca. Mace.

34. Ephydra rufitarsis. Id.

35. Piophila pusilla. MEIG.

30. - petesionis. Dus

37. Drosophila fasciata. Id.

38. maculata. Id.

SPHÉROCÉRIDES.

39. Sphcerocera subsultans. Mace.

40. Borborus equinus. Id.

$$
\text { IÉTÉROMYZIDES. }
$$

41. Gymnopa anea. FALL.

42. Agromyzu flara. MEIG.

$$
\text { UYPOCÈRES. }
$$

13. Phora pallipes. Latr.

44. - - bicolor. MACQ.

45. sordidipennis. DuF.

46. - livida. Id.

47. - aterrima. LATR.

48. -_helicivora. Dur.

Ainsi que je l'ai fait pour la famille précédente, je vais faire précéder mes descriptions anatomiques des acalyptérées de quelques observations isolées qui ne trouveraient pas place ailleurs.

1. Quoique le Chyliza et le Cordylura soient deux genres contigus dans la méthode de M. Macquart, il existe cependant entre eux, sous le rapport de leurs organes digestifs, une différence no- 
table, et celle-ci me semble de nature à appeler encore l'attention des diptérologistes sur leurs places respectives dans la classification.

2. Le Chyliza leptogaster a, dans les individus frais, la région dorsale du corselet couverte d'un fin duvet doré, soyeux, avec des raies obscures. Ce trait avait été saisi par Pauzer (Faun. Germ. fasc. 54, fig. 19). Les palpes sont très-noirs (caractère rare dans les Diptères), ovales-spatulés, presque sécuriformes. Les nervures des ailes sont testacées dans le mâle; noires, excepté à leur base, dans la femelle. L'abdomen est canaliculé en dessous dans les deux sexes.

3. Contre l'assertion de M. Robineau-Desvoidy les Scatophaga qui ont l'abdomen très-velu sont des mâles.

J'ai observé un fait accidentel fort singulier dans les deux sexes du Scat. stercoraria. A diverses reprises j’ai trouvé vers le milieu de la région ventralc de l'abdomen tantôt une, tantôt deux grandes ouvertures ovales qui communiquent dans la cavité abdominale et qui permettent d'apercevoir les viscères. Ces œils-de-bœuf étaient réguliers et nets, comme si on les eût faits avec un emporte-pièce. Ces mouches avaient l'air de se bien porter. Je présume que des larves parasites vivent dans l'abdomen de cette scatophage en s'y nourrissant seulement, soit du tissu adipeux, soit des sucs épanchés. Sans doute que ces larves pratiquent cette singulière opération césarienne lorsqu'elles sortent pour se transformer.

4. Le Platystoma umbrarum, Diptère d'un facies si hétéroclite, et l'une des plus grandes espèces des Muscides acalyptérées, présente une structure et une composition de la paroi ventrale de l'abdomen qui n'ont pas frappé les entomologistes. Cet insecte, qui a quatre segments dorsaux à l'abdomen, n'en a pas à la région ventrale dans l'un comme dans l'autre sexe. Cette dernière région est revêtue d'un derme homogène d'une seule pièce, qui n'est que le développement de la membrane inter-segmentaire ordinaire. Ce derme souple et d'un jaune citron est glabre et parcouru longitudinalement par de profondes stries qui ne sont que des rides 
ou de fines plissures. Mais comme la nature ne passe pas brusquement d'une création à une autre, une loupe scrupulcuse découvre encore les vestiges, les rudiments des segments disparus. Ainsi il y a tout à fait à la base une double plaque noire coriacée, puis au milieu une seconde très-petite, puis un ou deux points de cette couleur, enfin avant l'oviscapte une plaque carrée. Remarquez bien que ce défaut presque absolı de segments ventraux dans le Platystome n'est qu'un acheminement à leur disparition complète dans la famille des Pupipares, qui termine l'ordre des Diptères. J'ai déjà signalé ce même insecte comme formant une exception pour le nombre et la position des stigmates.

5. Les Diptères du groupe remarquable des Sepsidées exhalent une odeur parfumée des plus agréables, qui varie suivant les espèces. M. Macquart est je crois, le seul entomologiste auquel elle n'ait pas échappé et il la compare à celle de la mélisse. Le scalpel mª dévoilé l'organe qui prépare ces essences et je vais le décrire cn peu de lignes. La glande odorifique qui forme le trait anatomique le plus caractéristique des Sepsidées, existe dans les deux sexes. Elle s'insère sur la paroi dorsale du rectum dans l'intérieur duquel elle verse le produit de sa sécrétion. L'insecte expulse cette odeur par l'anus. Ce serait donc un organe des sécrétions excrémentitielles comparable á ceux que j’ai dejà fait connaître dans les Carabiques et autres Coléoptères, mais il en diflère surtout par son morle d'insertion sur le rectum. Il consiste en deux vésicules oblongues ou ovalaires, parfois d'une faible teinte roussàtre, où le microscope permet de constater une capsule intérieure de même forme. Elles s'atténuent un peu en arrière pour confluer aussitôt en un col d'une extrême brièveté, implanté à nu sur le rectum. Cette glande est, comme on voil, des plus simples. Il faut considérer les vésicules comme des organes sécréteurs par leurs parois, et réservoirs par leur cavilé, tandis que le col est le conduit excréleur. Cette odeur a en général du rapport avec l'éther, et dans le Cheligaster on croirait flairer l'éther acétique.

6. Les Sepsidées ont une mobilité dans les jambes et une promp11. 


\section{REGHERCIIES ANATONIOUES ET PHYSIOLOGIOUES}

titude dans le vol qui contrastent avec l'allure tranquille des autres Muscides acalyptérées. La première est favorisée par la longueur considérable des hanches, qui égale la moitié de la cuisse; la seconde par une composition des balanciers qui n'a pas encore été signalée. Ces organes, extrêmenent vibratiles, sont en petite massue insérée sur une base coliacée biarticulée.

7. L'abdomen du Cheligaster putris mâle se compose de cinq segments dorsaux. Le troisième, plus grand que ceux qui le précèdent et qui le suivent, est débordé près de ses angles postérieurs par une pièce tégumentaire de deux articles, terminée par un pinceau de plusieurs longues soies arquées qui, de part et d'autre de l'abdomen, s'inclinent réciproquement de manière à embrasser le bout de celui-ci. C'est ce trait sexuel qui a valu à cet insecte sa dénomination générique. La base articulée des pinceaux naît du troisième segment ventral.

La patte antérieure du mâle de cette même espèce est remarquable par le nombre de spinules et de soies qui garnissent le bord inférieur de la cuisse et du tibia. La cuisse a, dans sa moitié postérieure, deux crochets, dont l'antérieur seul est arqué, séparés par une échancrure arrondie. Le tibia a vis-à-vis celle-ci une saillie obtuse destinée à s'y loger, et vers sa base trois soies roides qui correspondent à de semblables de la cuisse. Ce membre est un organe de préhension redoutable. Je le crois exclusivement destiné aux manœuvres de la copulation. La cuisse de devant de la femelle a, au tiers antérieur de son bord inférieur, un peigne de six à sept soies très-rapprochées.

8. La pièce pénicillée du Chcligaster se retrouve, mais rudimentaire dans le Nemopoda cylindrica; au lieu d'un long pinceau, le mâle du Némopode a une touffe fort courle, une brosse de trèspetits poils roides.

Ce même sexe offre au segment ventral qui précède l'armure copulatrice un organe singulier qui combine son action avec le forceps lor's de l'union des sexes. Ce segment est unc plaque triangulaire partagée par une fente médiane favoráble aux mou- 
vements partiels de chacjue moitié. Aux angles de sa base s'insère une tige triarticulée, terminée par un peigne de quatre soies spimuleuses noires, qui s'incline vers son congénère pour former la pince. Cet organe existe aussi avec quelques légères modifications dans le Cheligaster.

9. L'Ulidia demandata se nourrit surtout des sucs fournis par les petites glandes pédicellées des sommités tendres des végétaux. Cette petite et brillante Muscide se complaît aussi dans la société des pucerons du parot oriental. J'ai fréquemment vu celte courtisane parasite lécher avec ses grosses lèvres les produits qui exsudaient des plaies faites par le bec des débonnaires Aphidiens. Dans son allure grave et compassée, elle meut ses pattes anté rieures à la manière de balanciers, comme pour palper et tâtonner au loin devant elle.

\section{CHAPITRE PREIIER.}

APPAREIL DIGESTIF.

Máagré la grande différence des Muscides de cette famille avec celles de la précédente, les organes de la digestion sont formés sur le même plan.

Les glandes salivaires sont ou uniformément capillaires, ou munies d'un réservoir terminal, rarement basilaire, parfois roulées en peloton.

Celles des Dolichocères sont pour la plupart longues, capillaires, flexueuses; mais dans le Sepedon sphegeus et les Tetanocera araloria et reliculata, elles sont renflées à leur extrémité. Dans le. Loxocera elles sont plus courtes, moins délices, avec un léger renflement terminal. On retrouve dans le Chiliza le réservoir basilaire du Lucilia, et dans le Cordylura le peloton des replis de l'Ech. grossa. J'ai dejà fait remarquer que ces deux formes anatomiques avaient le même but fonctionnel, celui du séjour et du perfectionnement de la salive. Cette organisation avancée des 
glandes salivaires dans les Cordylarides, me fait penser que cette sous-tribu pourrait bien avoir le pas sur celle des Dolichocères. Les Scatophaga et Hclomyza, qui ont plus d'un trait de ressemblance avec les Authomyzides, ont, comme celles-ci, des vaisseaux salivaires capillaires d'un bout à l'autre et de moyenne longueur. Il y a un réservoir terminal ovoïde dans les Sapromyza, les Ortalidées, le Tephritis, les Sepsidées. Dans les Ortalis, il y a, indépendemment de ce réservoir, une agglonération de quelques replis qui ne s'observe pas daıs le Platystoma, le géant de ce groupe. Le Calobata a, comme le Chiliza, dont il partage les habitudes, un réservoir salivaire à l'issue de la tête, tandis que le Micropeza, qui a avec le Calobata une si grande conformité de structure extérieure, a son vaisseau salivaire à peiné renflé au bout. L'Ulidia et les Lauxanides ont le réservoir terminal des Ortalis. L'Ochtera et le Notiphila ont la glande capillaire du Scalophaga. Dans le grouppe des Piophilides, il n'y a pas de conformité dans cet organe; les-Teichomyza, Piophila et Drosoph. maculata ont un réservoir terminal qui manque dans l'Ephydra et le Droscph. fasciata. Cette différence anatomique dans les deux Drosophiles viendrait à l'appui de l'observation que m’a faite M. Macquart, sur la nécessité d'établir une coupe particulière pourle Drosoph. maculata. Le petit grouppe des Sphérocérides présente deux formes très-différentes des glandes salivaires; dans le Spharocera, le corps de la glande est un boyau filiforme reployé, avec un col aussi long que lui, et de la ténuité d'un brin de soie. Dans le Borborus, la glande ressemble à celle du Myopa: c'est une bourse conoïde, dont la pointe éffilée ne dépasse pas le milieu du corselet, et dont la base arrondic a un col des plus capillaires. Cet organe est le caractère anatomique différentiel des deux genres. Les Hypocères ont des vaisseaux salivaires trèssimples, médiocrement longs et uniformément capillaires. Dans la Phora helicivora, ils sont insensiblement renflés en massue et atténués près de la tête en un col plus que capillaire.

Le tubc alimentaire differe peu par sa forme et sa composition rrénérales de celui des Anthomyzides el des Muscies. Il est filiforme, 
de deux à cing ou six fois plus long que l'insecte, par conséquent plus ou moins enroulé dans la cavité abdominale. Il débute, excepté dans un très-petit nombre d'espèces, par ce godet orbiculaire qui est le trait anatomique le plus caractéristique des deux familles de Muscides. Le réservoir de la panse est presque toujours bilobé et les vaisseaux hépatiques, à quelques rares exceptions près, ont deux canaux cholédoques plus ou moins marqués. L'intestin est filiforme et le rectum a quatre boutons charnus dans sa moitié antérieure : tels sont les traits généraux. Voyons les modifications suivant les petites peuplades établies sous le nom de sous-tribus par ${ }^{-} M$. Macquart.

Les vaisseaux hépatiques dans les Dolichocères ont une bile d'un brun violet foncé, et on trouve le réservoir de leur panse plus ou moins rempli d'un liquide brun ou roux. Cette dernière circonstance et l'habitat de ces Diptères indiquent assez quils doivent se nourrir de liquides qui tiennent en dissolution des matières décomposées. Le col de la panse offre dans le Loxocera un renflement conoïde à son insertion au réservoir. Celui-ci contient une liqueur de couleur ambrée, et les vaisseaux hépatiques sont d'un jaune pâle. Cet insecte ne vit pas enfoncé dans les plantes marécageuses, comme les Telanocera, et sa nourriture est sans doute plus épurée.

Le Chyliza et le Cordylura, quoique appartenant au mème groupe, ont dans la longueur respective de leur canal digestif des différences qui viennent à l'appui de ce que j’ai dit plus haut sur leur contiguïté générique, et qui annoncent que leur régime, et par conséquent leur genre de vie ne sont pas identiques. Cet organe n'a que deux fois la longueur du corps dans le Chyliza, il en a quatre ou cinq dans le Cordylura. Le réservoir de la panse est bilobé dans le premier, oblong dans le second. Dans l'un et dans l'autre, les vaisseaux biliaires sont d'un jaune pâle, et les canaux cholédoques, plus courts que dans les groupes précédents. Le rectum du Chyliza a quatre boutons charnus oblongs, et le Cordylara, six orbiculaires. Les Sapromyza ont un liquide alimentaire 


\section{RECHERCIES ANATOMIQUES ET PHYSIOLOGIQUES}

brun dans le réscrvoir de la panse et dans le ventricule chylificque, ce qui permet de croire qu'ils vivent de matières infusées. La bile est blanche dans les deux espèces. Un semblable liquide brun, trouvé dans l'organe digestif de l'Ilelomyza ligrina, dont la larve vit dans les champignons, prouve que l'insecte se nourrit de la matière décomposée de ccux-ci. La longueur du tube alimentaire des Ortalidées est moindre que dans les soustribus précédentes, et n'a pas plus de deux fois et demie celle du corps. Le réservoir de la panse est simple dans les Orlalis, bilobé dans le Platystoma. J'ai constaté dans celui des Ortalis un liquide vert foncé qui m’a semblé de nature végétale, et dans celui du Plalystoma une liqueur brune que l'insecte vomit aussi quand on le saisil. Il parait que ce dernier se nourrit de matière en décomposition. Le canal digestif des Sepsidées présente un trait anatomique qui leur est exclusivement propre, c'est l'existence sur le rectum d'une glande odorifique dont j’ai déjà parlé. Le réservoir de la panse renferme un liquide faiblement ambré dans le Nomopoda, et sa bile est blanche. Le tube alimentaire du Calobata et cèlui du Micropeza offrent des différences qui légitiment leur séparation générique. Il a trois fois seulement la longueur du corps dans le premier, et quatre ou cinq fois dans le second. Le réservoir de la panse est simple dans l'un, profondément trilobé ou même trifide dans l'autre. Les vaisseaux hépatiques sont sensiblement plus courts dans le Micropeza. L'Ulidia a le canal digestif aussi long que celui de ce dernier; le réservoir de sa panse est bilobé; les boutons charnus du rectum sont oblongs, et cette poche excrémentilielle a dans la femelle un long col qui témoigne de la longueur de son oviscapte. L'examen comparalif le plus minutieux ne sait découvrir d'autre différence entre l'organe de la digestion des Lauxanides et celui de l'Ulidia qu'une extrême brièveté des canaux cholédoques dans le Lonchcea. Ce même organe digestif a quatre ou cinq fois la longueur du corps dans les $\boldsymbol{H}_{y} d r o-$ myzides, Diptères qui se nourrissent de détritus. Les canąux cholédoques, qui ont la longueur ordinaire dans le Notiphila, sont 
fort courts dans l'Ochtera. Ce dernier insecte qui, par la struchure de ses pattes antérieures, semble destiné à vivre de rapine, a sur le rectum un organe insolite et manque des boutons charnus ordinaires. Cet organe consiste, pour chaque côté de la moitié antérieure du rectum, en une espèce de grappe irrégulière de quatre ou cinq tubercules ovales-conoïdes, d'une teinte jaunâtre, insérés sessilement à droile et à gauche d'une tige commune qui déborde la poche stercorale, et qui est peut-être un conduit. Je dis peut-être, car cette tige paraît charnue et compacte. C'est là un organe des sécrétions excrémentitielles qui a besoin d'être encore étudié et analomiquement et physiologiquement. Ce n'est pas une glande odorifique comme celle des Sepsidées. Je n'ai jamais remarqué que ce Diptère riverain exhalât une odcur particulière, ct les entomologistes ne nous apprennent rien de semblable.

Le groupe des Piophilides est loin d'avoir une organisation viscérale identique, et sa constitution générique devra, je pense, être remaniée. Le Teichomyza, qui la préside, et dont on doit la fondation et la découverte à M. Macquart, me semble devoir constituer un genre isolé. Son canal alimentaire est un des plus longs dans tout l'ordre des Diptères : il a cing ou six fois la longueur du corps. Le réservoir de la panse est bilobé, et je l'ai trouvé rempli, non pas d'un liquide, mais d'une bouillie blanchâtre. M. Nacquart nous apprend que cet insecte abonde sur les vieux murs humides des écuries et des latrines. Il est vraisemblable qu'en léchant avec ses larges et grosses lèvres le ciment imprégné de l'humidité alimentaire, il en avale quelques atomes, el de là cette pulpe blanche et opaque. Le ventricule chylifique, au lieu de débuter, comme dans toutes les Muscides précédentes, par un gorlet orbiculaire plus ou moins ombiliqué, commence, dans le Teichomyza, par un renflement ovalaire ou turbiné, tantôt plus, tantôt moins prononcé, dont les parois ont une consistance calloso-charnue. Ge renflement, ou mieux cet organe, débute trop brusquement pour être considéré comme une simple dilatation de l'oesophage et pour prendre le nom de jabot. Quoiqu'il ne m'ait présenté 
intérieurement aucune partie dure, aucune colonne calleuse, ainsi quion l'observe ordinairement dans un gésier, il doil cependant, à mon avis, être considéré comme une modificalion, un premier degré de celui-ci à cause de sa circonscription constante et de la consistance de ses parois. Certainement, la maticre alimentaire doit subir dans sa cavité une élaboration spéciale, ce qui vient a l'appui de la réflexion que m'a suggérée tout à l'heure la bouillie de la panse. Si je n'ai point découvert de valvule à l'orifice poslérieur dle ce gésier, oú je crois qu'elle existe, c'est que j’j manqué de sujets pour mes recherchęs. Cel insecte ne se rencontrant pas dans la contrée que j'habite, japportai moi-même de Bordeaux, en 1839 , les cinc ou six individus qui ont servi à mes vivisections, et ce n'était pas assez pour descendre dans les détails de structure intime. Quoi qu'il en soit, l'existence de ce gésier est le trait anatomique distinctif du Tcichomyza, au moins dans le groupe des Piophilides; car nous trouverons bientôt un semblable organe dans le Spherocera, qui a un genre de vie trés-analogue au sien. Après cet organe, le ventricule a la forme et les replis accoutumés. Les vaisseaux hépatiques et l'intestin ne diffèrent pas de ceux du type de la famille. Le rectum n'a pas un col prolongé, ce qui indique d'avance que le Teichomyza n'a plus un oviscapte prononcé.

Le canal digestif de l'Eplıydra est aussi long et même plus long que celui du Tcichomyza. Le réservoir de la panse est simple, ovoïde et peu développé. Il renferme une bouillie, signe d'affinité organique avec le genre précćdent; mais cette bouillie est jaunâtre. On sait que ces petites et innombrables Muscides habitent le limon des rivages ombragés et s'y nourrissent du détritus végéto-animal dont il est imprégné. Le ventricule n’a pas le gésier du Teichomyza, difl'érence anatomique capitale, et il offre le godet orbiculaire de presque toutes les Nuscides. Le reste comme dans le genre précédent.

Le Piophila, du mêne groupe, a le canal digestif à peine moins long que celui de l'Ephydra; mais, du reste, organisé sur le même plan. Le ventricule chylifique débute aussi par un godet 
orbiculaire; mais le réservoir de la panse est en bissac et rempli. non d'une bouillie, mais d'un liquide cristallin '.

Il y a dans le tube alimentaire du Drosophila fasciata des traits qui le rapprochent du Teichomyza et l'éloignent du Dros. maculala. Il a la longueur de celui des genres précédents. Le réservoir de la panse n'est pas bilobé; mais quand il est distendu, il parait arrondi, réniforme. Il renferme une liouillie roussâtre ou cannelle. Ce Drosophila vivant de substances végétales en fermentation, le ventricule offre, à son origine, un gésier turbiné semblable à celui du Teichomyza, et cette conformité anatomique est d'un piquant intérêt pour la classification. Lès vaisseaux hépatiques présentent une particularité qui nous prépare à la retrouver mieux caractérisée dans le genre Phora. C'est là un de ces organes de transition qu'il importe de signaler pour faire ressortir la marche graduelle des créations organiques. Ces vaisseaux, parfois remplis d'une bile blanche, ont un renflement vésiculaire terminal dans deux d'entre eux, ceux du côté droit, tandis que les deux autres sont uniformément capillaires. L'intestin est grêle comme un fil et le rectum est oblong.

Le canal digestif du Dros. maculata s'éloigne surtout par l'absence du gésier et des réservoirs biliaires du précédent et ressemble à celui du Piophila.

Le groupe des Sphérocérides, Diptères sautillants qui infesten! les bouses, les crottins et d'autres matières décomposées dont ils font leur nourriture, offrent comme les Piophilides de curieuses dissemblances splanchnologiques dans les deux seuls genres soumis à mes vivisections. J'ai déjà signalé celles qui existaient dans leurs glandes salivaires. Le tube alimentaire du Spharocera ressemble par sa longueur à celui du Piophila. Le réservoir de la panse est simple, ovalaire. Le ventricule a un gésier ovale-oblong, bien circonscrit, semblable à ceux du Teichomyza et du Dros. fasciata. Le Borborus n'a pas ce gésier, et il est pourvu du godet orbiculaire si commun aux Muscides. Sa bile est blanche.

' Voyez Métam. et anat. du Pioph. petasionis. (Annal. đles sc. nat. $3^{\circ}$ sér. t. I, p. 365.)

11. 
Le Gymnopa et l'Agromyza, les deux seules Hétéromyzides que j’aie disséquées, ont un tube digestif très-analogse à celui de l'Ulidia, dont il sernit peut-être prudent de les rapprocher.

Le canal alimentaire des Phora, de ces petits Diptères qui courent avec tant d'agilité, et qui semblent se nourrir des imperceptibles immondices répandues çà et là, offre la même composition générale que celui des autres Muscides; mais nous y trouverons aussi plusieurs traits de structure qui leur sont propres. Il a une longueur qui dépasse quatre fois environ celle de l'insecte. Il est un peu moins long dans le $P h$. sordidipennis, espèce qui nous fournira encore plusieurs caractères anatoniques qui revendiquent sa séparation du genre. L'esophage, bien plus long que dans la plupart des autres Diptères, traverse tout le corselet en conservant la ténuité du plus fin cheveu. La panse a une forme, une disposition insolites, variables dans les différentes espèces et dans tes individus d'une mème espèce, suivant son degré de plénitude, et d'autres conditions physiologiques d'une appréciation difficile. Je connais peu de Diptères où cet organe soit proportionnellement plus volumineux que dans les Phora. Le réservoir du pallipes est sessile ou presque sessile, tantôt réniforme, plus ou moins lobé au côté interne, avec un col excentrique si court, qu'il peut être contestable, tantôt trilobé comme un trèfle de cartes, ou simplement oblong et froncé. Il renferme une houillie blonde ou roussâtre. Je l'ai trouvé à trois lobes irréguliers dans le livida, grand, orbiculaire avec un col conoïde fort court dans le bicolor, globuleux, muni d'un col central capillaire plus long que lui, et renflé à son origine, dans le sordidipennis, où il était rempli d'un liquide cristallin un peu ambré. Cet organe a, dans l'helicivora, la forme d'une massue allongée très-simple. Le ventricule n'a, dans aucune espèce, son origine en godet orbiculaire comme dans les Muscides en général, el ce trait négatif est le caractère anatomique le plus saillant de ce groupe. Dans les pallipes, livida et bicolor, après l'insertion de la panse, il se continue directement en un tube filiforme replié en une ou deux circonvolutions. Celui de l'aterrima 
m'a semblé bifurqué à son origine; mais je sens le besoin de renouveler les autopsies. Je remarque dans le sordidipennis, qu'après l'insertion de la panse, l'osophage présente un petit renflement olivaire qui pourrait bien être un gésier. Le ventricule de cette espèce originale débute par une portion courbée en arc, et ce serait au milieu de sa convexité que s'implanterait le gésier. Il résulte de là que le ventricule se prolonge latéralement en un boyau borgne, en un cul-de-sac, en bout arrondi. Cette disposition est si insolite, que je me défie un peu de sa réalité. Comme je n'ai eu qu'un seul sujet à disséquer, je ne suis pas éloigné de croire qu'un coup de scalpel maladroit ou malheureux m'aura dérobé l'une des branches de la bifurcation de ce ventricule. Cette circonstance, si elle était fondée, rendrait alors plus vraisemblable la forme bilolée de l'origine de cet organe dans l'aterrima. Quoi qu'il en soit; le ventricule du sordidipennis ne se reploie qu'en une seule circonvolution, ainsi que celui de l'helicivora. Dans cette dernière espèce, qui a toute la structure extérieure du sordidipennis, l'origine du ventricule n'a aucune sorte de renflement.

Les vaisseaux hépatiques des Phora varient aussi suivant les espèces. Dans le pallipes et le bicolor, ils sont blanchâtres ou demidiaphanes, de médiocre longueur, et ont deux canaux cholédoques comme à l'ordinaire, mais ils offrent cette particularité que tous quatre ont un réservoir terminal vésiculaire ovoïde ou oblong. J'avais déjà signalé dans le Dros. fasciala l'indice d'une semblable configuration. Ce sont là des vésicules biliaires qui diffèrent surtout de celles des grands animaux, parce qu'elles sont apicales. Les vaisseaux biliaires du sordidipennis fournissent un nouveau trait anatomique qui milite pour sa séparation du genre Phora. Nonseulement ils n'ont pas les vésicules terminales, mais ils manquent de canaux cholédoques; leur quatre insertions sont isolées, quoique rapprochées. Ils sont aussi proportionnellement plus longs que dans les autres espèces. L'helicivora présente cette bizarre singularité, que deux des vaisseaux biliaires ont un canal cholédoque, et les deux autres en manquent. 
324 RECIERCHES ANATOMIQUES ET PHYSIOLOGIQUES

L'intestin des Phora est filiforme. Le rectum est souvent globuleux avec quatre boutons charnus, arrondis ou pyramidaux, el un col aussi long que lui.

CIIPITRE II.

APPAREIL GÉNITAL.

ARTICI E I ${ }^{\text {er }}$.

APPAREIL GÉnital MÂle.

Les testicules sont colorés en brun plus ou moins rougeâtre, comme ceux des Muscides calyptérées. Je ne connais d'autres exceptions que celles du Dros. fasciata et du Phora pallipes. IIs sont oblongs, fusiformes, dans la plupart des Dolichocères, mais plus gros et ovalaires dans les Sepedon sphegens et Tetanocera aratoria; d'une forme allongée, et courbés en hameçon dans le Loxocera, très-polymorphes dans le Chyliza, suivant le degré de leur turgescence séminale. Je les ai vus dans ce Diptère ou filiformes, flexueux ou courbés en crosse. Its sont ovoïdes, oblongs dans le Cordylura; ovales-oblongs ou fusiformes dans le Scatophaga slercoraria; en longs boyaux enroulés ou fléchis en anse dans les Orialis; moins longs et reployés en demi-spirale dans le Platystoma; gros et ovoïdes dans les Sepsidées, surtout dans le Nemopoda; de cette configuration dans le Micropeza; semblables dans l'Ulidia i ceux dẹ l'Ortalis, c'est-à-dire longs, filiformes, enroulés; ovoïdes dans les Lauxania, Teichomyza; allongés et de forme variable dans le Piophila. Le testicule du Dros. fasciata a une structure insolite, et n'a pas même cette couleur brune si générale dans les Muscides. Il est constitué par un vaisseau spermifique capillaire, blanchâtre, roulé en nombreuses spirales concentriques qui forment une rondelle plate. Le centre de celle-ci a une teinte jaune qui devient insensible ailleurs. Ce vaisseau, déroulé, est plus long que tout le Diptère, et son bout libre est renllé en massue. Cet organe est 
ovoïle, assez gros dans les Sphérocérides et le Phora pallipes, ou il est décoloré.

Les conduils déférents des testicules sont bruns comme ces derniers dans les Dolichocères, mais plus courts que la glande dans les deux Sepedon et le Tetanocera aratoria; plus longs dans les autres. Ils n'ont dans le Loxocera que l'étrécissement incolore du testicule, d'une excessive brièveté dans le Chyliza; plus màrqués dans le Cordylura; courts et plus ou moins décolorés dans le Scatophaga, dans les Ortalis, ou ils sont parfois fusiformes; d'une finesse uniformément capillaire et de la longueur du testicule dans le Platystoma; plus courts que ce dernier orgañe et de sa couleur dans les Sepsidées; presque nuls dans le Micropeza; moins longs que le testicule dans l'Utidia et le Teichomyza; bien plus courts dans le Piophila. Les testicules du Drosophila fasciata s'atténuent en arrière pour s'aboucher à un conduit déférent commun, capillaire, court, qui se fixe, non pas aux vésicules séminales, mais directement à l'origine du canal éjaculateur. Ce sont là deux modes insolices de connexions. Ces conduits dans les Sphérocérides sont plus courts que le testicule et décolorés; ceux des. Phora ont la longueur de cet organe et une ténuité capillaire.

Les vésicules séminales (ordinairement une paire, quelquefois deux) reçoivent les conduits déférents immédiatement avant l'origine du canal éjaculateur. Dans la plupart des Dolichocéres, elles sont filiformes et assez longues; plus courtes et un peu renflées au bout dans le Sepedon Ilacffnerii, avec un canal éjaculateur filiforme, long et flexueux. Il n'est pas facile de mettre en évidence celle du Loxoccra, à cause de leur contiguilté, soit entre elles, soit avec les testicules. Distendues à leur naissance, elles s'étrécissent en un boyau tubuleux courbé en anse. C'est par un col des plus courts qu'elles s'insèrent au canal éjaculateur. Celui-ci, filiforme et droil, présente cela d'insolite, que, bulbeux à son origine, il reçoit là le col capillaire d'une vésicule globuleuse inpaire, remplie d'une matière blanche. J'ignore entièrement les attributions physiologiques de cette vésicule, qui est peut-être un réservoir séminal 
supplémentaire. Il y a, dans le Chyliza, deux paires de vésicules séminales, l'une que j’appellerai essentielle, parce qu'elle reçoit les conduits déférents, est filiforme, longue, plus ou moins courbéc en anse, et parfois bifurquée; l'autre, analogue par sa configuration à celle du Loxocera, est renflée vers son origine, filiforme ensuile, et dirigée en arrière; mais elle varie encoresingulièrement, car je l'ai trouvée parfois presque globuleuse et placée en avant. Le canal éjaculateur est filiforme, assez long, bulbeux à son origine.

Les vésicules séminales du Scatophaga ont une gracilité capillaire et une médiocre longucur. Elles semblent plutôt s'insérer aux conduits déférents que leur fournir insertion. Le canal éjaculateur, qui égale en longueur le testicule avec son conduit déférent, est un peu en massue à son origine, puis il cst capillaire. Les vésicules des Orialis, blanchâtres et tubuleuses, sont au nombre de quatre dans l'Orl. vibrans, et de deux dans l'Ort. lugens. Celles de la première espèce sont plus courtes, une paire dirigée en avant, plus ou moins courbée en crosse et recevant les conduits déférents, l'autre paire dirigée en arrière. Celles de la seconde espèce ont une longueur presque double des précédentes, et sont plus ou moins reployées. Le canal ejaculateur, plus grêle que les vésicules, est un peu bulbeux à son origine dans l'Ort. lagens.

L'appareil génital mâle du Platystoma renchérit encore par son originalité et ses formes inaccoutumées sur les précédentes Muscides. C'est ici qu'il faut invoquer et toute l'acuïté de sa vue, secourue des lentilles amplifiantes, et l'indispensable labor improbus, pour démêler la simplicité et la symétrie au milieu de ce pêlemêle d'organes et de l'inextricabilité de leurs fragiles replis. Les vésicules séminales ont avec les conduits déférents des connexions tout a fait particulières, que je signalerai bientôt. Ces vésicules forment au-dessous d'un grand corps ovale central, analogue au réservoir séminal des Syrphies, un paquet, un plexus dont jai été assez heureux de dérouler les nombreux replis. Alors j'ai pu, à mon indicible satisfaction, mettre en évidence les connexions réci- 
proques des diverses pièces de l'appareil et y retrouver, avec quelques légères modifications, le type des organes masculins de l'ordre. Les conduits déférents aboutissent aux côtés de la convexité d'une utricule semi-lunaire transversale, organe insolite, sorte de sinus commun destiné à recevoir immédiatement le sperme sécrété, et à le transmettre, soit aux vésicules séminales, soit au canal éjaculateur. Ces vésicules naissent ou s'insèrent de chaque côté de la concavité de ce sinus. Ce sont deux vaisseaux simples, capillaires, semi-diaphanes, très-repliés, dont la longueur égale quatre ou cinq fois celle du corps de l'insecte. Le canal éjaculateur prend son origine à cette même concavité du sinus, cntre les insertions des vésicules. Il a presque la ténuité de celle-ci, est moins long qu'elle et très-flexueux. Il va s'insérer près du bout postéricur du réservoir séminal. Ce dernier est un corps ovalaire blanc, à parois consistantes et épaisses, situé au centre de tout l'appareil, qu'il recouvre en partie, et qui me semble destiné au séjour, à l'élaboralion du sperme, pour fournir sans doute à sa dépense, ou successive, ou intermittente pendant un coït, que je suppose, d'après la forme des organes copulateurs, devoir être et difficultueux et fort long. Le réservoir séminal communique avec l'armure copulatrice par un col court et gros.

Les vésicules séminales des Sepsidées sont filiformes, du double ai moins plus longues que le testicule. Indépendamment de celleslà, il y en a une impaire au milieu, tantôt en massue, tantôt ovoïde, et presque de la grosseur du testicule; comme elle ne se trouve pas sur le trajet du canal éjaculateur, je n'ai pas cru devoir lui donner le nom de réservoir séminal. Le canal éjaculateur est fort long, capillaire, flexueux, bulbeux à la base dans le Cheligaster. Il est moins long et renflé dans une partie de son étendue dans le Nemopoda. Il fait, à son origine, une boucle dans le Sepsis.

Les vésicules séminales de l'Ulidia sont plus que capillaires, fragiles, entortillées, et plusieurs fois plus longues que le corps, avec un canal éjaculateur fort court. Celui-ci est au contraire long 
et grêle dans le Piophila pusilla, où les vésicules sont filiformes et roulées en cercle. Dans le $P$. pctasionis, ces vésicules sont allongées, recourbées plus ou moins, renflées au bout, offrant avant leur confluence une utricule ovoïde, où s'implante le testicule. Cette utricule, malgré sa configuration, peut être prise pour un conduit déférent; le canal éjaculateur est long, plus ou moins dilaté à son origine. Les vésicules séminales du Drosoph. fasciata sont oblongues, assez grosses, insérées par un col presque nul aux côtés de l'origine du canal éjaculateur, en sorte que, pour devenir réservoirs du sperme, il faut que celui-ci y reflue de cette origine; le canal éjaculateur est en massue allongéc et médiocrement long. Il y a deux paires de ces vésicules dans les Sphérocérides: l'une, ovale ou ovale oblongue, reçoit les conduits déférents; l'autre, allongée, cylindroïde, ou à peine en massue. Le canal éjaculateur est tout à fait capillaire dans le Spharocera, bulbeux à son origine dans le Borborus. Les vésicules du Phora pallipes, de la grandeur du testicule, sont oblongues, cambrées, avec un col capillaire moins long qu'clles, offrant dans son milieu un pelit renflement sphéroïdal; c'est immédiatement avant celui-ci qu'a lieu l'insertion du conduit déférent; le canal éjaculateur est grèle, capillaire, assez long, flexueux.

Terminons ce qui concerne l'appareil génital mâle de nos petites Muscides, par le signalement des armures copulatrices de quelques-unes d'entre elles.

L'armure copalatrice du Loxocera, tout à fait collée sous le bout de l'abdomen, est fort petite; la pièce basilaire est un arc tégumentaire, ouvert en arrière, noirâtre, velu; un espace assez grand, occupé par des parties molles, mais bordé de chaque côté par une lame lorune, sépare cette pièçe du forceps, dont les branches sont lorunes, oblongues, obtuses, glabres, à peu près droites, un peu échancrées au bord externe; le fourrean de la verge est fin, allongé, d'un brun pâle, et semble se confondre à sa base avec une pièce transversale, qui lụi est peut-être commune avec le forceps. 
L'abdomen du Chyliza mâle est renflé ì son extrémité, qui est creusée en dessous d'une gorge profonde, à bords tranchants of droits, qui le prolonge dans toute la tongueur de cette partie et

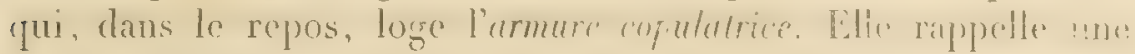
structure analogue dans les Libellules. La pièce basilaire est assez grande et arquée, comme dans le Loxocera, mais ses houts sont

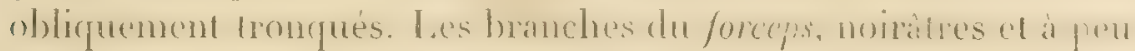
près droites, sont prolongées en pointe très-acérée; le fourrenu de la verge est' allongé, pâle, bordé dle baguettes étroites, brunes, terminées par un petit crochet tourné en dehors.

L'armure du Scalophaga est arrondie et logée sous le bout de l'abdomen; la pièce basilaire est coriacée, noirâtre, velue, large, at peine arquée; les branches du forceps consistent en deux crochets cornés, bruns, arqués, formant la pince par leur connivence. Je ne vois à la place du fourrcau de la verge que deux panneaux ovales, obtus, coriacés, velus.

L'armure des Ortalis est pareillement logée sous l'extrémité de l'abdomen.

Celle de l'Ort. vibrans consiste, $1^{\circ}$ en une pièce basilaire carrée,

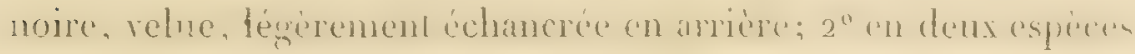
de tentacules oviles, bruns, velum, intecis en pétiole, sans apparrence de véritable forceps; $3^{\circ}$ en un filet élastique brun, plane, un fin ruban, d'abord droit, puis enroulé en une rondelle comme un ressort de montre : ce filet forme, en dehors du corps de l'insecte, une saillic constante sur un côté et vers la base de la pièce basilaire; l'une cle ses faces, l'interne, paraît au microscope loute couverte de soies assez longues, plus ou moins couchées; le pédicule seul est glabre. Je considère ce filet comme le fourreau de la verge.

La pièce basilaire de l'armure de l'Ort. lugens est plus échancrée en arrière que celle du vibrans; le forceps est évident; ses branches, distantes l'une de l'autre et presque droites, sont allongées, minces, de couleur ombrée, terminées par une dilatation transrersale, dont l'angle externe est obtus et crochu, et l'interne aigu. 
Entre ces deux angles, il y a une très-petite épine courbe; au milieu des bases du forceps, on voit deux pièces oblongues contiguës à la ligne médiane, analogues aux tentacules du vibrans. Comme dans cette dernière espèce, on trouve dans le lugens le Courreau de la verge enroulé hors du corps en ressort de montre, mais simplement bordé, d'abord, d'aiguillons triangulaires bien séparés, puis de piquants sétiformes allongés, susceptibles de se redresser et de s'incliner; enfin, ce ruban élastique est inerme un peu avant son extrémité, qui est terminée en pointe mousse.

Nous allons trouver dans l'armure copulatrice du Platystoma une recherche singulière de composition et de structure. Couchée dans le repos, dans l'excavation du bout de l'abdomen, elle consiste d'abord en deux pièces basilaires cornées, ñoires, glalıres; placées l'une à la suite de l'autre, et qui ne semblent que des segments tégumentaires qui auraient changé de forme et un peu de texture; la première, qui est ovale, convexe, tronquée en arrière pour s'articuler à la suivante, est arrondie en avant, où elle reçoit le col du réservoir séminal; la seconde pièce, analogue à celle des Orlalis, est presque carrée, un peu échancrée en arrière. Après cette échancrure, se voit le forceps, dont les branches écartées, mais susceptibles de se rapprocher pour former la pince, sont composées de deux pièces unies par une articulation : l'une est roussâtre; renflée, située à la base; l'autre est un crochet à trois petites dents tronquées, dont la plus interne est noire et l'externe roussâtre. Par une compression expulsive, exercée avec ménagement pendant la vie de l'insecte, on voit se présenter, entre les branches du forceps, une sorte d'étui assez grand, coriacé, membraneux, velu, qui ressemble au fourreau de la verge. Mais c'est dans l'étude du pénis. que nous allons voir se multiplier les prodiges de structure. De dessous et sur le côté de la seconde pièce basilaire, part, comme dans les Orlalis, un filet élastique.de couleur ambrée, d'une finesse qui surpasse celle d'un cheveu, et assez long pour faire une grande circonvolution sur lui-même. Ce filet, qui ne s'enroule pas en ressort de montre, 
aboutil à un corps fort gros, comparativement à lui, ovale-oblong, étranglé en calebasse, corné, très-glabre, lisse et luisant. Un peu avant son bout, libre et sous celui-ci, ce corps émet un filet de même nature et de même nuance que celui fixé au bout opposé, mais trois ou quatre fois plus court, et bifide à son extrémité. La plus forte lentille du microscope laisse aparcevoir, à travers la tunique ambrée et semi-diaphane du plus long filet, un tube inclus, et a la premiere partie de la calebasse une ce, sule pareillement incluse. L'írvocation de la loi de l'analogie mi norte à regarder ce filet comme un ćtui de la verge, ainsi que les grandes Tipules nous l'ont démontré, indis que le corps en calebasse doit être considéré comme un qland presque monstrueux. Il est permis de croire que ce gland est destiné à s'introduire, à se loger dans le vagin, a y demeurer tout le temps du coït. Il est probable que, pendant celui-ci, dont je n'ai pas été témoin, les deux sexes demeurent unis, attachés comme les chiens, et enfin qu'il se fait, durant cette union, plusieurs éjaculations.

L'armure des Sepsidées est, dans le repos, réfléchie et appliquée sous le bout de l'abdomen. Celle du Cheligaster a une circonscription ovalaire. Elle semble se réduire à une seule tenaille cornée ou forceps, laissant entre ses branches un grand vide, et susceptible d'un jeu assez étendu. Mais en y regardant de plus près, on reconnaît presque vers le milieu de ces branches, une petite acticulation transversale, ce qui permet de distinguer, $I^{\circ}$ une pièce basilaire cambréc, renflée, noire, luisante, un peu hérissée, unie en arrière à sa congénère par une fine articulation médiane, difficile à constater; $2^{\circ}$ une pièce terminale, plus grêle, plus pâle, pareillement cornée, un peu sinueuse, inclinée vers sa congénère pour faire la pince. Elle représente le crochet arqué et mobile qui s'observe dans quelques armures de Diptères et notamment dans le Sargus, le Leplis, etc. Au milieu de l'ouverture parabolique du forceps, se voit un étui oblong, corné, noir, glabre : c'est le fourreau de la verge; mais un fourreau, contre l'ordinaire, d'une seule pièce cornée, au moins vue par 
sa région dorsale. L'extrémité de ce fourreau émet, par une compression expulsive, un appareil assez compliqué, ou l'élude microscopique mia permis de constater, d'abord, un stylet central en crochet, modérément arqué, corné quoique pâle, avec une très-petite saillie dentiforme ver's sa base; puis, de chaque côté de cette dernière, une sorte de tubercule ou d'apophyse arrondie, veloutéc, dont l'une, plus saillante, est armée à sa racine d'un ergot corné, dirigć vers la souche de l'appareil. Je laisse à de plus habiles le soin de nous fixer sur les attributions respectives des diverses pièces de ce singtilier instrument copulatif.

L'armure du Nemopoda ressemble à la précédente, mais elle est plus arrondie, et je n'ai point saisi l'articulation mitoyenne des branches du forceps. Le fourreau de la verge est ici velu, échancré sur un côté. De son extrémité, sort un stylet aliongé, sinueux, hérissé.

Le trait anatomique qui établit l'affinité générique de l'Ulidia avec l'Orlalis, est l'existence, en dehors de l'armure copulatrice, d'un fourreau de la verge, sous la forme d'un filet enroulé comme un ressort de montre. J'ai décrit et figuré celte même disposition dans le Piophila petasionis.

L'armure du Drosophila fasciata ne manque pas non plus d'originalité. Elle a pour pièce basilaire un panneau ovalaire, de texture coriacée, couvert de longs poils et garni à son bord inférieur d'une série de dents cornées, brunes, dirigées les unes en avant, les autres en arrière. La plupart de ces dents, vues au microscope, ont leur pointe bifide. Au-dessous de ce panneau, est une plaque cornée, obłongue, subtriangulaire, finement dentelée à son bord tranchant. Cette plaque, quoique umique, remplacerait le forceps. Mais, ce qu'il y a de bien curieux, c'est qu'à plusieurs reprises jai pu constater, sur un insecte aussi petit, la verge parfaitement saillante. Cette verge (ou peut-être ce fourreau) est capillaire, assez longue, blanchâtre, et son bout libre offre au microscope une sorte de gland avec un très-petit crochet de chaque côté. 
L'armure du Spherocera est ovalaire et noire. La pièce basilaire est assez large, transversale, un peu arquée. Les branches du forceps sont pointues, médiocrement courbes, velues en dehors. Le fourreau se termine par deux stylets bruns sétiformes.

L'armure du Phora pallipes consiste principalement en un forceps coriacéo-corné, à branches grosses et hérissées de soies roides à leur base, étrécies ensuite pour se terniner par un cro chet en cuilleron arrondi, très-cilié en clehors. Le fourreau est glabre, cylindroïde, avec deux soies courbes à sa pointe. Ce dernier trait rappelle celui du Sphcerocera. Le microscope m'a fait découvrir, à la base du fourreau, deux plaques ovalaires ciliécs. appartenant peut-être à une volselle.

ARTIGLE II.

APPAREIL GÉNITAL FEMELLE.

Mes investigations anatomiques sur cet appareil ne miont pas mis à même de croire que dans les Muscides acalyptérées il existât des espèces vivipares, comme dans les calyptérées. Je n'y aj découvert aucun vestige de réservoirs ovo-larvigères. Les œuifs ì terme tombent dans le calice, quand il y en a, ou enfilent l'ovi ducte, pour séjourner, avaut d'être pondus, dans un renflemen de celui-ci, qui devient un réservoir ovigère, analogue à colui don les calyptérées nous ont fourni plusieurs exemples. Je me bor nerai à choisir, dans le grand nombre d'ovaires que j'ai étudiés ceux dont les modifications, de forme ou de structure, peuven servir de types pour l'ensemble de la famille.

Les ovaires du Sepedon sphegeus et du Tetanocera stictica con sistent en deux grappes ovalaires-conoïdes de vingt-cing it trent gaînes ovigères multiloculaires. Le calice est postérieur et plu: ou moins cupuliforme. Le col est fort court, et l'oviducte long el filiforme, sans réservoir ovigère. Les œufs à terme sont oblongs et blancs. Je n'ai su apercevoir, pour appareil sébifique et sémina 
du Sepedon, que deux boyaux filiformes plus ou moins boursouflés, d'une légère teinte roussâtre, renflés en arrière en une vésicule ovalaire qui s'insére par un petit col à l'extrémité de l'oviducte. Malgré des recherches réitérées, je n'ai pas découvert des orbicelles, comme dans d'autres Dolichocères, et je sens le besoin de reprendre ces autopsies.

J'ai trouvé dans les Telanocera aratoria el stictica, trois orbicelles à centre noir, munis de cols éfférents capillaires. Dans la première de ces espèces, il existe aussi deux réservoirs utriculaires ovales atténués en col.

L'oviscapte des Dolichocères est nul ou presque nul, et c'est l'indice que ces Diptères n'introduisent pas, mais déposent leurs neufs. Les derniers segments dorsaux du Sepedon sont étroits, engaînés de manière à se prêter, lors de la ponte, à des mouvements variés et à un certain allongement. Il y a deux tentacules vulvaires d'un seul article ovalaire velu.

Les ovaires du Loxocera ont la configuration de ceux des Dolichocères, mais ils n'ont chacun que dix à douze gaînes ovigères allongées, tri ou quadriloculaires, ce qui explique la rareté de ces Diptères comparativement aux Sepedons. L'oviducte présente, peu après son origne, un réservoir ovigère allongé, à parois épaises et consistantes. L'appareil sébilique et séminal ne m'a offert que deux orbicelles à centre roussâtre, subsessiles, et un seul réservoir ovoïde pédicellé. L'oviscaple est petit, d'une seule pièce tubuleuse brune et grêle. Il insère sans doute les aufs dans quelque milieu résistant.

Ceux du Chyliza ont encore la forme des précédents, avec une quinzaine environ de gaines ovigères multiloculaires. L'oviducte a un réservoir ovigère, analogue à celui du Loxocera. Les oufs à terme sont allongés et blancs. L'oviscaple est formé aussi d'un seul tuyau rétractile, terminé par deux tentacules vulvaires, d'un seul article. Les réservoirs séminaux sont ovales, avec un long col.

Dans les Scatomyzides, les ovaires du Sapromyza ustulata ont 
un nombre indéterminable de gaînes.ovigères multiloculaires, et l'oviducte offre, comme les précédents, un réservoir ovigère. La glande sébifique a trois orbicelles ronds à centre noir, munis d'un très-long col; le Sap. rorida n'en a que deux, mais l'un a deux taches noires, et l'Helomyza rufa a deux longs cols avec un double orbicelle à chacun.

Les ovaires du Plalystoma, dans un état de fécondation avancée. sont gros, subglobuleux, blancs, hérissés dans tous les sens par une quantité innombrable (plusieurs centaines) de gaînes ovigères allongées, très-serrées, biloculaires, terminées par un ligament propre. Le calice, qui, dans les groupes précédents, était postérieur, est ici central; te col fort court, ainsi que l'oviducte, jusqu'à l'insertion de la glande sébifique, car après celle-ci il se prolonge pour suivre les mouvements de l'oviscapte. Remarquer qu'il n'y a pas de réservoir wigère. Les oufs sont allongés, grêles, pointus par un bout.

Dans les individus vierges, les ovaires sont plats, triangulaires, et les cols s'insèrent à l'angle postérieur et interne du triangle.

L'appareil sébifique et séminal, situé à peu de distance de l'origine de l'oviducte, a trois orbicelles ronds à centre noir, qui paraissent sessiles dans la situation normale des organes, mais qui, dans le fait, ont un très-long col efférent capillaire. Ces orbicelles sont tellement enlacés dans un tissu adipeux membraniforme, qu'il est fort difficile de les mettre en évidence. Les réservoirs séminaux sont deux boyaux allongés plus ou moins renflés vers leur milieu, flexueux, remplis d'une matière trèsblanche. C'est surtout dans les individus non fécondés que les connexions de tous ces organes peuvent être bien saisies.

L'oviscapte est long et formé par deux tuyaux bruns, parcheminés, rentrant l'un dans l'autre, et dont le postérieur, plus grèle, se termine par quelques poils. Je n'ai pas aperçu des tentacules vuluaires. L'oviscapte, dans le repos, s'engage dans le cône tronqué noir, luisant, non rétractile, du bout de l'abdomen qui lui sert d'étui. 


\section{;36 RECHERCHES ANATOMIQUES ET PHYSIOLOGIQUES}

Les ovaires de l'Ortalis lugens ressemblent à ceux du Plalyitoma par leur configuration et le nombre des gaines ovigères. Vhais celleś-ci m'ont paru tri ou quadriloculaires, et l'oviducte, après a glande sébifique, présente un réservoir ovigère oblong, à parois épaisses, comme dans la plupart des groupes précédents, ce qui éveille un peu mes doutes sur son absence dans le Plat)stoma.

Les ovaires des Sepsidées présentent, suivant leur état ou leur degré de fécondation, des différences insidieuses de forme et de structure apparentes. Aussi faut-il, pour ramener ces différences au lype normal, multiplier à l'infini les autopsies aux diverses époques de la vie.

Dans le début de la gestation, les ovaires sont ovales ou ovalesoblongs, couverts de toutes parts de granulations arrondies, el au côté interne de chacun d'eux on remarque, tantôt plus, tantôt moins près de leur extrémité, un ligament comme fibreux ou strié, dirigé en avant. Dans cet état, les gaînes ovigères sont tellement agglomérées et confondues, qu'il est impossible de saisir leur forme propre. Par les progrès de la gestation, la configuration et même la structure éprouvent un changement considérable, une sorte de mélamorphose. Lorsque.l'évolution complète de l'organe est arrivée, lorsque les aufs sont à terme dans leurs gaînes, l'ovaire forme une sorte de rondelle comparable à celle des Muscies. La tranche de la rondelle présente une série circulaire et serrée te gaines ovigères innombrables et uniloculaires, remplies d'œuls allongés, tandis que de son disque creux, excavé; part un faiscean pyramidal el effilé, formé par les longs ligaments suspenseurs propres de ces gaînes, et allant se fixer dans le thorax. J'ai domné la figure de ces deux états dans le Cheligaster.

Le calice de l'ovaire est postérieur, c'est-à-dire formé par l'évasement du col, du moins je l'ai constaté ainsi dans un Nemopoda qui avait peut-être déjả commencé sa ponte, el oủ, par conséquent l'origine du col s'était dilatée pour recevoir les ouf́s à terme. Celte dilatation était nulle dans l'ovaire du Cheligaster que j’ai re- 
présentée. Le col est court, l'oviducte grêle, filiforme, aussi long que l'ovaire. Cet oviducte se perd en arrière dans un corps allongé à parois résistantes qui est le réservoir ovigère. Les œufs sont allongés, grêles, olstus, blanchâtres.

L'appareil sébifique et séminal s'insère immédiatement avant le réservoir ovigère. Il y a dans le Cheligaster trois orbicelles, dont deux à longs cols, et l'autre séssile. Ce dernier et l'un des autres ont un centre noir qui n'existe pas dans le troisième. Je n'ai pas aperçules réservoirs séminaux, et je suis porté à croire qu'ils ont échappé à mes regards. Dans le. Nemopoda, oủ les trois orbicelles sont tous pédicellés, et où un seul d'entre eux a le centre noir, j’ai trouvé un réservoir séminal ovalaire, mais un seul, ce qui n'est pas ordinaire.

L'oviscapte du Cheligaster m'a paru formé d'un seul tuyau allongé et rétractile. J'ai vu sortir par son bout deux crochets noirâtres à peine arqués, destinés sans doute à saisir ou à diriger les ceuf́s lors de la ponte. Ces crochets ne sont pas des tentacules vulvaires; ceux-ci existent de chaque côté du dernier segment de l'abdomen sous la forme d'une fine et longue spatule.

Je n'ai étudié les ovaires des Leptopodites que dans le Calobata. Dans une gestation avancée, ils sont ovales, triangulaires, déprimés, mais plus ou moins fléchis sur eux-mêmés, pour se prêter à l'étroitesse de l'abdomen. Its sont composés d'un nombre incalculable de gaînes ovigères allongées, tri ou quadriloculaires, ce qui annonce que cette espèce doit pulluler beaucoup. La face inférieure de l'ovaire est dégarnie de gaînes, excepté sur ses bords, en sorte que le calice est inférieur, le col est.court, et l'oviducte aussi. Les œufs à terme sont allongés, pointuś par un bout, blancs. Je n'ai constaté de la glande sébifique que deux orbicelles dépourvus de centre noir, mais avec de longs cols efférents.

Les ovaires de l'Ulidia ressemblent beaucoup à ceux de l'Ortalis, et surtout du Platystoma. Parvenus à leur dernière période de gestation, ils sont ovales, obtus, garnis d'une quantité prodigieuse (de centaines) de gaînes ovigères, uni ou biloculaires très- 


\section{RECHERCHES ANATOMIOUES ET PHYSIOLOGIOUES}

serrées, paraissant alors globuleuses, parce que les bouts des œufs qu'elles contiennent sont sursaillants. Le calice est postérieur, et le col fort court. L'oviducte est long et forme, bientôt après son origine, une double anse dilatée et d'une consistance calleuse. C'est un réservoir ovigère. Les æufs à terme sont ovales-oblongs, blancs.

L'appareil sébifique et séminal a, dans l'Ulidia, une composition différente de celles que nous avons étudiées jusqu'a ce jour. Les orbicelles à centre noir sont au nombre de trois, avec de longs cols capillaires. Deux d'entre eux ont un pédicule commun, mais chacun d'eux a un col court. Le troisième conflue avec les deux autres pour former un conduit excréteur assez court, qui s'insère sous l'origine du renflement ovigère. Les réservoirs séminaux sont deux utricules pyriformes, avec un long canal excréteur capillaire.

Les ovaires de l'Ochtera, au temps d'une gestation avancée, sont volumineux et ovalaires. Les gaines ovigères, au nombre de quinze à vingt, sont allongées quadri ou quinqueloculaires. Les cufs à terme sont remarquables par leur couleur noirâtre, leur grandeur, leur forme oblongue, cambrée parfois, allénuée à un bout, et les fines stries longitudinales de leur surface. Le microscope y révèle une enveloppe diaphane. Leur structure annonce quelque particularité dans leur destination.

Ovaires du Teichomyzu et du Drosophila: ovales-conoïdes, composés chacun d'une vingtaine cnviron de gaînes ovigères, allongées, quadriloculaires. Col assez court, oviducte filiforme, flexueux; plus long que l'ovaire.

L'appareil sébifique et séminal est inséré, non à l'orighine, mais ì l'extrémité postírieure de l'oviducte. Ofbichles ghohuleux. subdiaphanes, dépourvus de centre noir, de consistance un peu calleuse, munis d'un col capillaire à peine de leur longueur. lié-

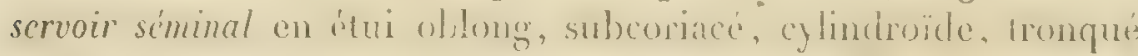
au bout, avec son axe faiblement roussâtre, et un col grêle plus court que lui. 
Ovaires du Piophila petasionis : en rondelle subarrondie, comme hérissée, à sa paroi supéricure, d'une quarantaine de gaînes ovigères, courtes, subtriloculaires; calice inférieur, col bien marqué. Oviducte gros, expansible, offrant un cul-de-sac latéral (bourse copulatrice d'Audouin), giande sébifique à un seul orbicelle à large centre noir, sessile; deux paires de réservoirs séminaux, l'une ovoïde, pédicellée, l'autre en boyau allongé, courbé, inséré par un col capillaire. Oviscapte de trois tuyaux engaînés.

Ovaires du Borborus: ovalairẹs composés d'une vingtaine environ de gaînes ovigères bi ou triloculaires, courtes, grosses, insérées sui la paroi supérieure de l'organe; calice par conséquent inférieur. Col court, oviducte assez long et filiforme. Ce dernier se reploie d'abord en une circonvolution, puis il augmente un peu de diamètre et ses parois deviennent consistantes pour former un réservoir ovigère. OEufs ovales, obtus.

L'appareil sébifique et séminal s'insère au bout de l'oviducte après le réservoir ovigère, ce qui est insolite. Deux orbicelles à centre noir, ovalaires, à col plus court que de coutume, renflé à sa naissance: Autour de ces orbicelles, on voit les replis asseznombreux d'un ou peut-être de deux tubes blanchâtres, capillaires, dont je n'ai pas constaté les counexions, et qui correspondent aux reservoirs.

Ovaires du Phora pallipes: dans une fécondation avancée, oblongs, oltus, composés de vingt à vingt-cinq gaînes ovigères, uni ou peut-ètre biloculaires, peu serrées, affectant une disposition en séries longitudinales. Calice postérieur, col court, oviducte grêle, assez long. OEafs à terme, ovales, obtus, blancs. Ovaires du Sordidipennis ayant l'originalité anatomique des autres viscères. Quoique la femelle où je les ai étudiés fût dans un état de grossesse à terme, et que le développement des ovaires eût envahi presque toute la cavité abdominale, chacun d'eux n'était composé que de cinq gaînes ovigères subbiloculaires, ce qui prouve la rareté de cette espèce. Les weufs sont gros, ovales-arrondis, blancs. Col 
bien marqué, ainsi que l'oviducte. L'appareil sébifique et séminal offre, relativement à son point d'insertion, une exception dont le Borborus nous a déjà fourni un exemple. Il s'insère à la partie postérieure de l'oviducte. Il n'y a pour organe sécréteur qu'une grosse vésicule ovoïde subdiaphane remplie d'un liquide roussâtre. Deux vaisseaux simples capillaires reployés, insérés aux côtés de la vésicule, représenteraient les réservoirs séminaux.

\section{FAIILLE DES PUPIPARES.}

Le fait original qui a domné son nom à cette famille, la dernière de l'ordre des Diptères, est acquis à la science depuis le heau mémoire que Réaumur consacra à l'illustration de l'Hippobosque ${ }^{1}$, et qui n'a laissé rien à apprendre sur le genre de vie et les métamorphoses de cet insecte.

Les Pupipares devaient aussi exciter un vif intérêt sous le rapport de leur anatomie, tous les méthodistes s'étant accordés à les reléguer au dernier degré de l'échelle diptérologique, il devenait curieux de constater cette décadence organique. En 1825 , j’ai publié, sur l'Hippobosca equina, mes recherches anatomiques². Je les ai renouvelées depuis, et je vais en donner un extrait en $y$ ajoutant quelque chose sur le Melophagus.

Espèces disséquées:

1. Hippobosca equina. L.

2. Melophagus ovinus. Latr.

3. Ornithomya viridis. $\mathrm{L}$.

L'absence totale des segments à l'abdomen, tant à la région dorsale qu'à la ventrale, est, dans les Pupipares, un caractère de structure extérieure d'autant plus intéressant à signaler, que la transition avait dejà été préparée par le Plalysloma, où le ventre seul est dépourvu de segmentation. Je ne me lasse pas d'appeler

$2 M e m .14$, t. VI, pl. 48

${ }^{3}$ Annal. des sc. nat. t. VI. p. 299, pl. 13. 
l'admiration sur cette marche successive de la nature dans les créations. C'est une loi universelle. Le tégument abdominal de l'hippobosque est épais, coriacé, mais susceptible d'une grande extension. Il est doublé d'un muscle peaucier dont la contractilité s'exerce puissamment à l'époque de l'accouchement. Les poils y sont implantés chacun sur une éminence arrondie.

\section{GHAPITRE PREMIER.}

IPPAREIL DIGESTIF.

Ces insectes se nourrissent du sang des animaux vivants dont ils sont parasites.

Les glandes salivaires sont plus compliquées, mieux organisées que dans beaucoup d'autres insectes. L'organe sécréleur occupe la base de la cavité abdominale. Il consiste, dans l'hippolosque etl'ornithomye, en un boyau flexueux, et dans le mélophage, en une capsule subglobuleuse. Il communique par un col efférent très-capillaire à un réscrvoir membraneux, orbiculaire dans le mélophage et l'ornithomye, ovoïde dans l'hippobosque, situé vers le milieu du thorax. De ce réservoir, part brusquement un canal excrétcur très-fin, plus ou moins reployé, confluent avec son congénère en un conduit commun, qui verse la salive dans la bouche.

Le tube alimentaire est le plus étendu de tous ceux des Diptères, puisqu'il a huit à neuf fois la longueur de l'insecte. Celui des derniers genres des acalyptérées nous avait déjà préparés à la progression croissante de la longueur de ce canal, avec la progression décroissante de l'organisation. C'est là un fait curieux à consigner. La privation absolue de la panse est encore un fait négatif d'un piquant intérêt, quand on se rappelle que le genre Phora qui, dans la série, précède immédiatement les Pupipares, nous avait fourni des variations de cet organe qui témoignaient hautement des incertitudes organogéniques et de la tendance à la disparition de ce premier réservoir digestif. L'csophage est court 
et très-fin. Le ventricule chylifique, de la longueur des trois quarts de tout le tube, débute dans le thojax par un renflement ovalaire un peu calleux, un jabot, plutót qu'un gésier. Il offre, dans l'abdomen, des boursouflures variables plus ou moins gorgées de sang; puis il devient filiforme, et s'enroule en plusieurs circonvolutions.

Les vaisseaux hépatiques n'ont pas de canaux cholédoques, et ont leurs quatre insertions isolées. La bile est souvent, comme dans les Phora, d'un blanc amylacé. L'intestin, grêle comme un fil, débute par un renflement en godet, ce qui est un trait anatomique propre à l'hippobosque. Le rectum a quatre boutons charnus arrondis. Ceux-ci sont des muscles papilliformes.

\section{CHAPITRE II.}

APPAREIL GENITAL.

ARTICLE Ier.

APPAREIL GENITAL MALE.

Les testicules sont fauves à l'extérieur, comme ceux du plus grand nombre des Muscides; mais ils ont une structure fort rare dans les Diptères, oú cependant les Asiles et les Conops nous en ont fourni des exemples assez analogues. Ils consistent chacun en une agglomération des nombreux replis d'un vaisseau spermifique simple, subcapillaire, qui, déroulé, a quatre ou cinq fois la longueur du corps de l'insecte. Parmi les Coléoptères, les Carabiques ont de semblables testicules. Ceux du Drosophila fasciata, Diptère de la famille des Muscides acalyptérées, en approchent pour la longueur du vaisseau spermifique, mais non pour sa disposition. Le conduit déférent n'est que la continuation du testicule Il y a une paire de vésicules séminales capillaires, mais chacurie d'elles, peu avant son insertion, se divise en deux branches simples. Le canal cjaculatcur est fort gros, comparativement aux autres parties. Il est conoïde, et s'atténue en arrière pour devenir fili- 
forme; à peu près droit dans le mélophage et l'ornithomyie; reployé dans l'hippobosque. L'armure copulatrice a un forceps à deux lames droites contiguës dans le repos. Le fourreau de la verge se termine par deux petits tentacules.

\section{ARTICLE II.}

APPAREIL GÉNITAL FEMELLE.

Les Pupipares n'accouchent ni d'œufs ni de larves, mais d'une chrysalide ou pupe sous la forme apparente d'un œuf démesurément grand. Ce mode de parturition exigeait une organisation exceptionnelle de l'appareil générateur, et dans l'immense nation des insectes, il n'existe aucun autre exemple d'un semblable fait. Cel appareil se compose des ovaires, de l'oviducte, d'une matrice, d'une glande sébifique et de réservoirs séminaux.

Les ovaires ne sont plus, comme dans les autres insectes, deux faisceaux de gaînes ovigères uni ou pluriloculaires. Chacun d'eux est une capsule simple, monosperme, ovoïde. L'un est constamment plus petit que l'autre, parce qu'ils ne sont pas fécondables en même temps. Arrondis à leur bout libre, ils s'atténuent èn zm col pour s'aboucher à l'oviducte. Dans mes nombreuses autopsies, je n'y ai jamais rencontré un véritable œuf. J'y ai vu un embryon qui ne tarde pas à revêtir l'ébauche de la pupe elle-même. Mais cet embryon, à l'époque de sa maturité, loin de se détacher comme un ceuf pour tomber dans l'oviducte, tient encore à l'ovaire par un cordon ombilical qui te tie anatomiquement et physiologiquement avec le corps de la mère.

La matrice est un organe creux ovalaire, blanc, éroinemment contractile el expansible, s'abouchant, d'une part, à l'oviducte, de l'autre sessilement à la vulve. Elle est destinée à recevoir le produit de la conception. Celui-ci est toujours unique, et mérite le nom de fotus. Dans les premiers temps de la gestation, il demeure encore suspendu au cordon ombilical. Par le progrès de son développement, il rompt ses liens avec sa mère, et acquiert une vie 
privée, individuelle. Celle-ci s'élablit, se maintient par l'emprunt à la matrice d'un appareil circulatoire trachéen, d'un système vasculaire nutritif que le fotus s'approprie définitivement pour vivre de sa vie propre et indépendante. Il est déjà à cette époque pupe, et à cquelque phase de la fécondation que jaie éludié cet insecte, jamais je n'ai pu y constater ni un $x u f$ ni une larve. Ainsi la pupe ou chrysalide semble exister a conceptu. C'est là un fait unique en entomologie, et inédit jusqu'à ce jour. Je développerai, dans un mémoire spécial dont je coordonne les matériaux, l'anatomie et la physiologie de ce piquant organisme.

Le produit de la parturition ou la pupe offre, dans les divers Pupipares, des différences de forme et de structure dont je réserve l'exposition pour le mémoire dont je viens de parler.

La glande sébifique a pour son organe pair de sécrétion un arbuscule rameux à tronc distinct, à cime plus ou moins agglomérée. Les deux troncs, avant leur insertion à l'oviducte, confluent en une souche commune fort courte. Les rameaux et les ramuscules de cette curieuse glande ont un filet tubuleux inclus, mais leur enveloppe est peu ou point contractile, tandis que le tronc et les canaux qui vont y verser le produit de la sécrétion, ont, et une tunique extérieure musculaire et un tube axal strié en travers ou mieux annelé.

Les réservoirs séminaux, pareillement doubles, sont insérés un peu en avant de la glande sébifique. Dans le mélophage, c'est une bourse simple et oblongue, dans l'hippobosque, un tube rameux à rameaux courts, inégaux et rares. 


\title{
EXPLIGATION DES FIGURES,
}

\author{
TOUTES CONSIDÉRABLEMENT GROSSIES.
}

Fig.

1. Système nerveux de Tipula oleracea.

aa. Hémisphères cérébraus étalés et rétines oculaires.

b6. Optiques et rétines ocellaires.

c. Portion du tube digestif avec l'oesophage engagé dans le collier rsophagion.

d. Ganglions thoraciques soudés.

ce. Ganglions abdominaux et paires de nerfs qui en naissent.

2. Systeme nerveux de la larve de $\mathbf{X} y$ phara atrata.

aa. Hémisphères cérébraux étalés.

b. Portion du tube digestif avec l'cesophage engagé dans le collier osophagien.

c. Ganglions thoraciyues contigus. dd. Ganglions abdominaux,

3. Système nerveux de Tabanus bovinus.

aa. Lobes du cerveau vus par-dessus et recouverts par ies choroides.

b. Bulbe rachidien, avec le collier œsophagien marqué d'un trait.

c. Ganglion thoracique avec ses paires de nerfs.

d. Ganglions abdominaux, idem.

4. Cerveau de ce Tabanus, renversé pour mettre en évidence ses hémisphères enchatonnés dans les rétines oculaires.

5. Choroide détachée et renversée pour
Fig.

faire voir les trachées de sa face rélinéenne.

6. Système nerveux de Volucella $\approx 0$ naria.

aa. Cerveau renversé et étalé. On y voit ses hémisphères enchatonnés dans les rétines et les trois optiques ocellaires avec leurs choroides.

b. Portion du canal digestif, avec l'aesophage engagé dans le collier aesophagien.

c. Ganglion thoracique avec ses paires de nerfs.

d. Ganglions abdominaux avec leurs nerfs.

7. Système nerveux ganglionnaire de Conops rufipes mâle.

a. Ganglion thoracique

b. Ganglion abdominal.

8. Système nerveux ganglionnaire du Conops rufipes femelle.

a. Ganglion thoracique.

b. Ganglion abdominal beaucoup plus distant du précédent que dans le mâle.

cc. Longs filets nerveux simples qui n'existent pas dans le mâle.

d. Division du cordon inter-'ganglionnaire, qui est simpledans le mâle. 


\section{IRECHERCHES ANATOMIQUES E'T PHYSIOLOGIQUES}

Fig.

9. Système nerveux de Calliphora vomitoria.

"a. Hémisphères cérébraux étalés, bordés par la choroide.

b. Nerfs buccaux et antennaires partaut de deux mamellons cérébraux.

c. Bulbe rachidien et collier aesuphagien marqué par un trait.

d. Ganglion unique, thoracique, avec ses paires de nerfs.

e. Cordon nerveux médian avec ses paires de nerfs.

10. Hémisphère cérébral détaché el choroide.

11. Nerfs et rétines ocellaires isolés.

12. Appareil respiratoire de la larve de Mycethophila inermis, nombre et disposition des stigmates et des Irachées.

aa. Stignates thoraciques.

bb. Stigmates alsdominaux.

13. Appareil respiratoire de larve de Tipula lunata.

aa. Stigmates vus par leur face interne pour faire voir le paren clayme trachéen.

14. Un de ces stigmates vu par sa face externe.

15. Profil du Tabanus bovinus, nombre et disposition des stigmates.

aa. Stigmates thoraciques, l'antéricur mésothoracique, le postérieur métatlıoracique.

b. Stigmates abdominaux; inter-segmentaires.

16. Un stigmate thoracique détaché de ling.

ce Tabanus, pour faire voir sa siruclure, sa valve ciliée.

17. Abdomen de Lucilia Casar vu par sa face inférieure avec le nombre et la disposition des stigmales abdominaux; segmentaires.

18. Tête et appareil digestif du C'nlex annulatus fomelle.

a. Tête avec ses antenıes à poils verticilles, ses palpes, sa trompe.

b6. Glandes salivaires.

ic. Bourses ventriculaires.

d. Panse.

e. Ventricule chylifique.

ff. Vaisseaux hépatiques.

9. Rectum précédé de l'intestin.

h. Derniers segments dorsaux de l'abdomen.

19. Origine du lube digestif dece Cnlex, vue par-dessous pour inontrer en $a a$ les bourses ventriculaires en $b$, une forme particulière de la panse.

20. Appareil génilal mâle du même Culex.
aa. Testicules.
bb. Conduits déférents.
cc. Vésicules séminales.
d. Armure copulatrice vue par des. sus.

21. Arnure copulatrice vue par dessolls.

au. Branches du forceps.

b. Volselle.

22. Appareil génital femelle de ce Cu. lex.

aa. Ovaires fécondés.

b. Orbicclles de la glande sébifique. 
Fig.

$\therefore$ Réservoir séminal.

d. Dernier segment dorsal avec les tentacules vulvaires.

A. Gaine ovigère détachée, uniloculaire.

13. OLufs de diverses formes.

c. Un orbicelle isolé avec son centre noir et son col efférent.

1). Tentacule vulvaire isolé.

23. Tête el appareil digestif de Tipula oleracea femelle.

t. Tête horizontale avec les antennes, les palpes et les parties de la bouche étalés.

bb. Glandes salivaires.

. Panse

1. Veatricule chylifique.

$\therefore$ Vaisseaux hépatiques.

1. Rectum précédé de l'intestin.

$\therefore$ Bout de l'abdomen et oviscapte.

1. Portion de l'antenne pour faire voir sa composition et sa structure.

F. Une palpe isolée : structure sémiarticulée du dernier article.

C. Portion du tissuadipeux splanchnique.

1). Glandes salivaires détachées.

a. Cols efférents.

1. Conduit excréteur.

E. Extrémité du ventricule clytifique.

$\therefore$ Insertions des vaisseaux hépatiques.

b. Origine de Inintestin.

F. Configurations particulières du réservoir de la panse.

24. Glandes salivaires fort longues de Macrocera hybrida.

25. Appareil génital mâle de Tipula olerucera.
Fig.

it. Testicules.

1h. Conduits déférents.

1. Vésicules séminales el canat éja. culateur.

i. Bout de labdomen et armure copulatrice.

26. Portion de ce même appareil isolée, déroulée et étalée.

ac. Testicules.

bb. Renflements épididymiques des conduits déférents.

c. Cordon ou fourreau renfermant une partie des conduits déférents.

dd. Vésicules sćminales.

e. Caual éjacułateur.

f. Fourreau de la verge avec la vésicule centrale insolite, et la menbane byaline.

A. Extrémité tricuspidée du fourreau de la verge.

27. Arınure copulatrice de la même Tipula, vue par sa face inférieure avec toutes ses parties constitutives élalées.

a. Plaque ventrale refendue au milieu, et formant le réceptacle de l'armure.

bb. Pièces latérales consistant dans les opercules, tes crochets du forceps, les baguettes, la volselle en lames de sabre, et deus stylets.

. Pièces centrales; à sa base une trifide, puis la verge.

A. Verge isolée; gland bifide.

28. Appareil génital femelle de Tipula oleracea.

u. Ovaires avec les cufs dे terme noirs. 
Fig.

bb. Cols des ovaires.

c. Ligament suspenseur.

d. Vésicules sécrétoires de la glande sébifique.

e. Réservoirs.

A. Gaîne ovigère isolée.

B. Réservoirs isolés.

29. Appareil génital femelle du Ceroplatus dispar.

aa. Ovaires.

bb. Cols.

c. Glande sébifique.

d. Bout de l'abdomen et oviscapte.

A. Gaine ovigère, uniloculaire.

B. Lame de l'oviscapte.

30. Glande sébifique de ce Ceroplatus, vue par-dessous pour montrer

a. Les organes sécréteurs avec leurs cols.

b6. Les réservoirs.

c. Le bulbe de l'oviducte.

dd. Les cols des ovaires.

31. Bout de-1'abdomen et armure copulatrice du mâle.

32. Tête et appareil digestif de T'ri. choplera trifusciata femelle.
a. Tête avec antennes et palpes étalées.
b. Panse.
c. Ventricule chylifique.
dd. Vaisseaux hépatiques et vésicules biliaires.
c. Intestin et rectum.
f. Bout de l'abdomen.
A. Portion de tissu adipeux splanch. nique.

33. Portion de l'organe digestif isolée, pour faire voir une variété à cinq vaisseaux hépaliques.
Fig.

34. Appareil génital mâle de Trichopteru ocellaris.

ar. Testicules.

bb. Vésicules séminales.

cc. Autre paire de ces vésicules.

d. Armure copulatrice et bout de l'abdomen.

A. Brancle du forceps isolée.

B. Branche de la volselle isolée.

35. Testicule et vésicules séminales isolés, pour mettre en évidence leurs connexions.

a. Testicule à peine atténué au con. duit déférent.

b. Vésicule séminale plus grande.

c. Autre vésicule plus petite.

d. Canal éjaculateur.

36. Tête et apparcil digestif de la larve de Tipula lunala.

aa. Glandes salivaires.

b. OEsoplage.

c. Gésier.

dd. Bourses ventriculaires.

e. Ventricule chylifique.

ff. Vaisseaux bépatiques.

g. Coecum latéral.

h. Intestin et rectum.

i. Lambeaux mésentériformes criblés du tissus adipeux.

37. Tête et appareil digestif du Tabunus tropicus femelle.

a. Tête avec antennes, palpes et trompe très-étalées.

bb. 'Glandes salivaires.

c. Panse.

dd. Bourses ventriculaires.

c. Ventricule chylifique en partie granuleux terminé par quelques papilles isolées.

ff. Vaisseaux hépatiques. 


\section{Rectum et intestin. \\ h. Dernier segment dorsal de l'ab. domen.}

38. Glandes salivaires isolées de Tabarus bovinus.

aa. Cols efférents.

b. Conduit excréteur.

39. Portion du canal digestif du $T^{\prime} a b$. bovinus, vue par la surface inférieure, pour montrer les connexions.
a. OEsophage un peu rentlé.
b. Panse avec son réservoir vide, plissé, festonné et l'insertion de son cul à la terminaison de l'oesophage.
$c c$. Bourses ventriculaires avec liga- ment terminal.

40. 'Tête détachée et trompe très-ćtalée de Pangonia marginata femelle. pour mettre en évidence sa com. position.

a. Fourreau ou étui de la trompe.

bb. Les valves.

cc. Les lancettes.

d. L'hypoylosse.

e. La langue.

41. Appareil génital mâle de Tabanus ater.

a. Testicules.

bb. Conduits déférents.

cc. Vésicules séminales.

d. Canal éjaculateur.

c. Dernier segment dorsal de l'abdomen.

f. Armure copulatrice.

A. Forme particulière du testicule de cc même tabanus.
Fig.
B. Portion de cet appareil génital renversée.
au. Insertions des conduits délé rents aux vésicules séminales et confluence de colles-ci pour former le canal éjaculateur.
C. Branche du forceps isolée.
I). Pièce biarticulée.
E. Fourreau de la verge.

42. Appareil génital femelle du Tabanus bovinus.
aa. Ovaires avancés dans la gresta- tion.
b. Ligament suspenseur.
c. Orbicelles de la glande sébi- fique.
dd. Réservoirs.
c. Hectum.
f. Dernier segment dorsal rle l'abdomen.
g. Tentacules vulvaires.
A. Gaîne ovigère isolée.
A. Orbicelle avec son col elférent.

43. 'Tète et appareil digestif d'Ephippium thoracicum.

a. Tête et antennes.

bb. Glandes salivaire.

c. Panse.

dd. Bourses ventriculaires.

c. Ventricule chylifique.

f. Vaisseaux Lépatiques avec un seul canal cholédorque.

gg. Deux des vaisseaux hépatiques plus gréles.

h. Intestin débutant par un renflement.

i. Rectum.

j. Bout de l'abdomen terminé par les tentacules vulvaires.

k. Portion de l'organe dorsal. 
350 RECHERCHES ANATOMIQUES ET PHYSIOLOGIQUES

Fig.

44. Glandes salivaires de Strationys chanceleon.

45. Appareil digestif de Vappo palli. pennis.

aa. Glandes salivaires.

h. Panse.

cc. Bourses ventriculaires.

d. Ventricule chylifique.

c. Vaisseaux hépatiques avec un canal cliolédoque unique.

f. Deux de ces vaisseaux plus courts rudimentaires.

1. Intestin.

1. Rectum.

1. Vésicule de la glande sébifique du lappo.

46. Appareil génital mâle de Sargus

cuprarius.

ana. Testicules.

bb. Conduits déférents.

cc. Vésicules séminales.

d. Canal éjaculateur.

e. Armure copulatrice établie.

A. Portion isoléc d'une branclie du forceps avec son crochet velu.

I3. Fourreau de la verge isolé avec ses appendices.

47. Appareil génital mâle de Beris vallata.

aa. Testicules.

bb. Vésicules séminales.

c. Canal éjaculateur.

d. Armure copulatrice vue pardessus.

48. Cette même armure isolée est vue par-dessous.

a. Tube du canal éjaculateur.

b. Piece basilaire.

cc. Branches du forceps.
Fig.

dd. Lames qui accompagnent le fourreau de la verge.

49. Portion isolee de ce même appareil génital.

a. Testicule.

b. Conduit déférent agggloméré.

cc. Portion des vésicules séminales.

d. Canal éjaculateur.

50. Appareil génital mâle d'Odontomyia tigrina.

ua. Testicules.

b. Peloton des conduits déférents agglomérés.

cc. Insertion de ces conduits aux vésicules séminales.

dd. Vésicules séminales.

e. Canal éjaculateur.

f. Armure copulatrice à branches du forceps forles, hérissées au dehors et au dedans; à fourreau de la verge court tronqué.

51. Appareil génital femelle de Beris vallata.

aa. Ovaires.

b. Oviducte.

c. Orbicelles.

dd. Réservoirs séminaux.

e. Tentaculesvulvaires bi-articulés.

A. Gaine ovigère et oufs de ce Beris.

B. Orbicelles et cols efférents lléchis au même point.

52. Tête et appareil digestif de Dasypogon Teutonus femelle.

a. Tête avec antennes, palpes et trompes étalées.

6b. Glandes salivaires.

c. Panse.

dd. Bourses ventriculaires.

c. Ventricule chylifique. 
$f^{\prime \prime} g$.

ff. Vaisseaux liépatiques.

g. Rectum avec ses boutons char. nus et l'intestin grêle.

h. Bout de l'abdomen et tentacules vulvaires.

53. Appareil digestif d'Asilus crabroniformis.

ut. Glandes salivaires, cols efférents et conduit excréteur.

b. OEsophage.

'. Bourses ventriculaires. Il n'y a pas de panse.

d. Ventricule chylifique asec sa portion récurrente.

‘V Vaisseaur hépatiques.

f. Rectum.

A. Forme singulière de la glande salivaire.

54. OEsophage isolé pour faire voir sa structure singulière et son mode insolite d'implantation an ventri. cule chylifique.

55. Appareil génital mâle de Laphriu fulva.

a. Testicules dans leur scrotum.

b. Vésicules séminales.

ir. Forceps copulateur.

d. Harpon et pièce carrée de la volselle.

B. Pièce réceptaculaire de l'armure vue par sa face inférieure et convexe, avec deur faisceaux de soies et deux appendices spatulés.

C. Fourreau de la verge isolé, terminé par une pointe bifide.

56. Teslicules dégagés du scrotum.

aa. L'un roulé en tire-bouchon, l'autre déroulé:
Fig.

b6. Vésicules séminates.

c. Canal ejaculateur.

57. Appareil génital mâle de Dasypogon Tentonus.

ar. Testicules

bib. Vésicules séminales.

c. Armure copulatrice.

A. Crochets de l'armure.

58. Appareil génital mále d'Asilus crabroniformis.

aa. Testicules.

bb. Vésicules séminales.

c. Canal éjaculateur.

d. Armure copulatrice.

59. Celle armure vue par sa face inflerieure.
a. Pièce basilaire.
$b b$. Branches du forceps.
$c c$. Volselle.
d. Fourreau de la verge.

(j0. Appareil génital femelle d'Asilus crabroniformis.

a. Ovaire dans sa situation naturelle.

b. Ovaire détaché et renversé pour faire voir le calice qui est in. férieur.

c. Ce calice.

d. Organe sécréteur de la glande sébifique.

єє. Késervoirs.

f. Rectum.

g. Oviscapte et lentaculesvulvaires.

A. Gaine ovigère multiloculaire.

B. Un des organes sécréteurs de la glande sébifique, isolé.

61. Appareil génital femelle d'Empis livida.

ar. Ovaires. 


\section{RECHERCHES ANATOMIQUES ET PHYSIOLOGIQUES}

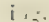

b. Organe sécréteur de la glande sébifique.

cc. Réservoirs séminaux.

d. Bout de l'abdomen, oviscapte et tentacules vulvaires.

A. Gaine ovigère isolée.

62. Têle et appareil digestif de Bombylins minor.

a. Tête, antennes et trompe.

bb. Glandes salivaires.

c. Panse.

dil. Bourses ventriculaires.

c. Ventricule chylifique.

ff. Vaisseaux hépatiques.

g. Rectum et boutons charnus.

h. Bout de l'abdomen.

A. Antenue isolée.

B. Glandes salivaires isolćes.

63. Appareil génital mảle de Bombylius posticus.

aa. Testicules.

5. Conduils déférents.

c. T'ésicules séminales.

d. Canal éjaculateur.

e. Armure copulatrice.

A. Branche du forceps, isolée.

64. Appareil génital femelle de Bomby. lius cruciatus.

aa. Ovaires.

b. Orbicelles.

cc. Réservoirs.

d. Bout de l'abdomen et tentacules vulvaires.

65. Appareil sébifique et séminal de ce Bombylius, étalé.

a. Orbicelles et cols efférents.

bb. Réservoirs vésiculaires.

cc. Réservoirs capillaires.

\section{rig.}

66. Bout de l'abdomen de Bombylius major.

a. Peigne.

A. Pointes ou baguetles isolées de ce peigne.

67. Orbicelle détaché de ce même Bombylius, avec le trait brun de l'axe de son col.

68. Appareil génital mâle de Thereva plebeia.

aa. Testicules.

$b b$. Vésicules séminales.

c. Canal éjaculateur.

d. Armure copulatrice.

69. Armure copulalrice vue par sa face inférieure.

ar. Pièces basilaires.

bb. Stylets.

cc. Forceps et volselle.

d. Fourreau de la verge.

70. Tête et appareil digestif du Leptis tringaria mâle.

a. Tête, antennes, palpes et parties de la bouche étalées.

bb. Glandes salivaires.

c. Panse.

$d d$. Bourses ventriculaires.

$e$. Ventricule chylifique.

ff. Vaisseaux hépatiques.

g. Intestin avec un renflement de son origine, et le rectum.

h. Bout de l'abdomen.

A. Glande salivaire détachée.

71. Appareil génital mâle de Leptis tringaria.

aa. Testicules.

bb. Conduits déférents.

cc. Vésicules séminales. 
Fig.
d. Canal éjaculateur.
e. Dernier segment ahdominal.
f. Armure copulatrice:
g. Branche du forceps isoléc.
h. Fourreau de la verge.

72. Appareil génital femelle du Leptis tringaria.
aa. Ovaires.
b. Glande sébifique.
cc. Réservoirs séminaux.
d. Oriscapte.

73. Tête et appareil digestif de Dolichopus nitidus.
a. Tête placée horizontalement.
bb. Glandes salivaires.
c. Panse.
dd. Bourses ventriculaires.
$\epsilon$ Ventricule chylifique.
$f f$. Vaisseaux hépatiques.
g. Rectumet intestin.
h. Dernier segment abdominal.
A. Antenne isolée.
B. Diverses configurations du réser- voir de la panse.
C. Glande salivaire isolée.

74. Appareil génital mâle de Dolichopus nitidus.
an. Testicules.
bb. Conduits déféreuts.
cc. Vésicules séminales.
d. Canal éjaculateur.
e. Armure copulatrice.

75. Lamelle isolée de l'armure copu. latrice.

76. Appareil génital femelle de ce Dolichopus.
aa. Ovaires.
b. Cols des ovaires.
c. Glande sébifique.

11 .
Fig.

dd. Réservoirs séminaux.

c. Oviscapte.

A. Gaine ovigère isolée.

B. Col efférent de l'organe sécréteur. sébifique.

C. Réservoir isolé.

D. Bout isolé de l'oviscapte.

77. 'T'êle et appareil digestif de Volucella zonaria.

a. Tête vue Lorizontalement.

$b b$. Glandes salivaires.

c. Panse.

dd. Bourses ventriculaires

c. Ventricule chylifique.

ff. Vaisseaux hépatiques.

g. Rectum et intestin.

h. Dernier segment dorsal de l'ab. domen.

78. Portion de cet appareil pour faire voir les connexions.

aa. Glandes salivaires.

b. Panse avec son col inséré à la terminaison de l'cesophage.

cc. Bourses ventriculaires appendiculées.

d. Ventricule chylifique.

79. Tête et appareil digestif de Rhingia rostrata.

a. Tête vue horizontalement.

bb. Glandes salivaires.

c. Panse.

dd. Bourses ventriculaires.

e. Ventricule chylifique.

$\int f$. Vaisseaux hépatiques.

g. Rectum et intestin.

$h$. Bout de l'abdomen.

80. Portion de cet appareil renversée pour meltre en évidence l'insertion de la panse et la forme des bourses à digitations égales. 
Fig.

81. Appareil génital mâle de Volucella zonurive.

ar. Testicules et conduits déférents.

bi. Vésicules séminales.

c. Canal éjaculateur renflé en réservoir spermatique.

d. Armure copulatrice.

A. État particulier des testicules de cette même espèce.

B. Portion de l'armure copulatrice constituant l'hypotome.

C. Baguette isolée du fourreau de la verge.

82. Appareil génital femelle de cette Volucella.

$a u$. Ovaires.

b. Orbicelles.

cc. Rćservoirs séminaus.

d. Oviscapte.

c. Rectum.

A. Gaine ovigère isolée.

B. Orbicelle avec son col efférent.

S3. Appareil sébifique (et séminal) de l'Eristalis tenax.

aa. Arbuscules des réservoirs.

b. Orbicelles.

cc. Cols des ovaires et aufs.

d. Oviducle,

84. Tête et appareil digestif de Scenopinus fenestralis.
a. Tête vue horizontalement, avec un trait transversal aux yeux.
bb. Glandes salivaires.
c. Panse d̀ réservoir simple.
dd. Bourses ventriculaires.
e. Ventricule chylifique.
ff. Vaisseaux hépatiques.
g. Intestio.
h. Rectum.

Fig.

i. Derniers segments dorsaux de l'abdomen.

1. Portion du canal digestif isolée pour mettre en évidence le mode d'insertion et de connexion des vaisseaux hépatiques.

85. Appareil génital mâlc de ce Scenopinus.

ua. 'l'esticules.

bb. Vésicules séminales.

c. Canal éjaculateur.

d. Armure copulatrice.

86. Appareil génilal femelle de ce Scenopinus.

aa. Ovaires.

b. Cols des ovaires.

cc. Orbicelles et conduits efférents.

d. Dernier segment abdominal et tentacules vulvaires.

A. Réservoir séminal.

B. Orbicelle détaché.

C. Gaîne ovigère isolée.

87. Tềte et appareil digestif de Myopa ferruginea.

a. Téte vue horizontalement, avec antennes, palpes, trompe.

b6. Glandes salivaires.

c. Panse avec deux configurations dilférentes du réservoir.

d. Ventricule chylifique.

ce. Vaisseaux hépatiques.

f. Intestin et rectum.

88. Glandes salivaires isolées de ce Myopa.

89. Appareil génital mâle de Stachyniđ meridionalis.

aa. Testicules.

bb. Vésicules séminales. 
c. Canal éjaculateur.

d. Armure copulatrice.

90. Fourreau de la verge isolé.

31. Abdomens accouplés de Conops rufipes.

92. Appareil génital mâle de Conops rufipes.

aa. Testicules.

bib. Vésicules séminales.

c. Canal éjaculateur.

d. Armure copulatrice.

93. Tête el appareil digestif de Cephalemyia ovis.

a. Tête vue horizontalement.

b. OLsophage.

c. Panse.

d. Ventricule chylifique.

e. Vaisseaux hépatiques remarquables par leur grosseur.

f. Intestin et rectum.

g. Bout de l'abdonen et anus.

94. Appareil génital måle d'Otstrus equi.

ua. Testicules.

bb. Vésicules séminales.

c. Canal éjaculateur.

d. Armure copulatrice.

95. Tête et appareil digestif d'Hypoderma bovis, larve.

a. 'Tète vue horizontalement avec les diverses aspérités et les parties de la bouclue.

$b b$. Glandes salivaires.

c. Panse.

d. Ventricule chylifique.

$\therefore$. Yaisseaux hépatiques.

f. Intestin.

g. Partie postérieure de l'abdomen, avec les deux stigmates.

h. Portion de lorgane dorsal.
Fig.

96. Tèle et apparoil digestif d'Echinomilin yrrissu

a. Tête, antennes, etc.

$b h$. Glandes salivaires avec un peloton de replis.

c. Panse.

d. Ventricule chylifique, avec son origine en godet orbiculaire.

ec. Vaisseaux hépatiques.

J. Intestin.

g. Rectum avec ses boutons charnus.

h. Dernier segment dorsal de labdomen.

1. Portion de l'antenne.

13. Portion boursouflée d'un vais. seau hépatique.

97. Partie de l'appareil génilal màle de cette Echinomyia.

ar. Testicules.

$b b$. Conduits déférents.

cc. Vésicules séminales.

d. Canal éjaculateur.

98. Appareil génital mâle d'Echinomyiı rubescens.

aa. Testicules avec les conduits déférents.

bb. Vésicules séminaleq.

c. Canal éjaculateur.

d. Armure copulatrice.

99. Appareil génital mâle de Sericocerı compressa.

aa. Testicules et conduits déférents.

bb. Vésicules séminales.

c. Canal éjaculateur.

d. Armure copulatrice.

A. Voiselle.

100. Appareil génital femelle d'Echinomyia grossa.

aa. Ovaires en rondelle ou plateau. 
350

Fig.

b. Réservoir ovo-larvigère.

c. Appareil sébifique.

d. Rectum.

A. Lambeau du réservoir ovo-larvi. gère avec les œufs fixés à la paroi interne.

B. Une gaine ovigère isolée.

101. Portion détachée de ce même appareil vu par sa face infé. rieure.

na. Les ovaires.

$b b$. Les cols des ovaires.

c. L'oviducte.

d. Les orbicelles.

ce. Les réservoirs séminaux.

f. Portion du réservoir ovo-larvigère.

102. Appareil génital femelle du Dexia rustica.

na. Ovaires.

b. Appareil sébifique et séminal.

c. Réservoir ovo-larvigdre.

A. OEuf isolé.

103. Appareil sébilique et séminal isolé de ce même insecte.

n. Orbicelles.

bb. Réservoirs séminaux.

104. Larve de Dexia prise dans le réservoir ovo-larvigère.

105. Appareil génital femelle de Gym. nosoma rotundata.

ac. Ovaires.

b6. Cols des ovaires.

c. Oviducte.

d. Orbicelles.

ę. Réservoirs séminaux.

A. OEufs. rig.

106. Tête et appareil digestif de Prosena sibirica.

a. Téte, antennes, trompe, etc.

bb. Glandes salivaires.

c. Panse.

d. Ventricule chylifique.

ee. Vaisseaux Lépatiques.

$f$. Intestin et rectum.

g. Bout de l'abdomen.

107. Appareil génital femelle de Prosena.

un. Oraires.

b. Glande sébifique.

c. Réservoir ovo-larvigère.

d. Bout de l'abdomen el tentacules vulvaires.

A. Une gaîne ovigère.

108. Appareil génital mâle de Sarcophaga hemorrhoidalis.

an. Testicules.

b. Conduits déférents.

cc. Vésicules séminales.

d. Canal éjaculateur.

e. Armure copulatrice.

f. Crochets du dernier segment ventral.

A. Fourreau de la verge isolé pour faire voir ses crochets.

B. Crochet en spatule arrondie.

109. Appareil génital femelle de Sarcophaga.

aa. Ovaires.

b6. Réservoirs oıo-larvigères pleins de larves.

c. Appareil sébifique et séminal.

d. Larves sortant du corps.

e. Rectum.

110. Ce même appareil renversé pour 
Fig.

faire voir les connexions des parties constitutives.

na. Ovaires avec leurs cols.

b. Réservoirs ovo-larvigères vides.

c. Oviducte.

d. Glande sébifique.

111. Appareil sébilique et séminal isolé et étalé.

aa. Orbicelles.

$b b$. Réservoirs séminaux.

112. Tête et appareil digestif de Lucilia Cersar.

aa. Tète vue horizontalement.

$b b$. Glandes salivaires avec réservoir et peloton.

c. Panse.

d. Ventricule chylifique.

ee. Vaisscaux Lépatiques.

f. Interlin.

g. Rectum.

h. Derniers segments abdominaux.

A. Portion des glandes salivaires pour mettre en évidence les réservoirs et le canal excréteur.

113. Appareil génital mâle du même Lucilia.

$a a$. 'Testicules et conduits déférents.

$b b$. Vésicules séminales.

c. Canal ejaculateur.

d. Armure copulatrice.

$e$. Pièces du dernier segmeut ventral.

114. Appareil génital mâle de Calliphora vomitoria.

aa. Testicules avec leur tunique accessoire.

b. Vésicules séminales.

c. Canal ejaculateur.

d. Armure copulatrice.
Fig.

115. Le même appareil plus grand.

air. Testicules sans leur tunique accessoire, avec leurs conduits efférents.

b. Vésicules séminałes.

c. Canal ejaculateur.

d. Armure copulatrice.

116. Appareil génital mâle de Lispa tarsalis.

aa. Testicules.

3b. Vésicules séminales.

c. Canal éjaculateur.

d. Armure copulatrice.

c. Pieces du dernier segment ventral.

117. Appareil génital mâle de Curtonevra maculata.

aa. Testicules avec leurs conduits déférents.

b. Canal éjaculateur à flectuosités qui tiennent lieu de vésicules séminales.

c. Armure copulatrice.

118. Appareil génital femelle de Lucilia Ceesar.

$a a$. Ovaires en rondelle.

bb. Appareil sébifique et séminal.

c. Rectum.

119. Appareil génital màle de Chyliza leptogaster.

ua. Testicules.

$b b b b$. Vésicules séminales.

c. Canal éjaculateur.

d. Armure copulatrice.

120. Le même appareil vu jar-dessous pour montrer les connexions de ses parties.

aa. Testicules turgescents. 


\section{RECHERCHES ANATOMIQUES ET PHYSIOLOGIQUES}

habb. Vésicules séminales.

c. Canal éjaculateur bulbeux d̀ son origine.

121. Apparcil génital mâle de Ścato. phaga siercoruria.

nuau. Testicules avec des configurations diverses.

h. Vésicules séminales.

c. Canal éjaculateur.

d. Armure copulatrice.

122. Appareil génital mâle de l,oxocera ichneumonea.

ra. T'esticules.

bh. Vésicules séminales.

c. Vésicule impaire.

d. Armure copulatrice.

123. Appareil génital mâle d'Ortalis vibrans.

aa. Testicules.

bl. Deux paires de résicules séminales.

c. Canal éjaculateur.

d. Armure copulatrice.

c. Fourreau de la verge roulé en ressort de montre.

A. Le méme fourreau déroulé.

124. Portion de l'appareil génital mâle d'Orlalis lugons.

a. Testicule.

b. Vésicule séminale.

c. Canal éjaculateur.

d. Armure copulatrice.

e. Fourreau de la verge.

B. Branche du forceps isolée.

C. Fourreau de la verge en partic déroulée pour faire voir sa structure.

125). Aplareil genital màle de l'las?stoma umbrarum.

aa. Testicules et conduits déférents.
Fis.

c. Vésicules séminales.

bb. Réservoir séminal.

d. Armure copulatrice.

c. Fourrean de la verge.

f. Gland.

126. Le même appareil déroulé et étalé.

an. Testicules.

bb. Conduits déférents.

cc. Vésicules séminales.

d. Sinus semi-lunaire.

c. Canal éjaculateur.

f. Réservoir séminal.

127. Portion du fourreau de la verge el gland pour faire voir la siructure.

A. Portion de l'armure copulatrice vue de profil.

128. Appareil génital mâle de Cheligaster putris.

aa. T'esticules.

bb. Vésicules séminales.

c. Vésicule impairc.

d. Canal éjaculateur.

e. Armure copulatrice.

A. Fourreau de la verge isolé.

129. Tête et appareil digestif de $\mathrm{Ne}$ mopoda cylindrica femelle.

a. Tête.

bb. Glandes salivaires.

c. Panse

d. Ventricule chylifique.

ce. Vaisseaux hépatiques.

f. Intestin.

gg. Glande odorifique.

h. Rectum.

i. Derniers segments dorsaux de l'abdomen.

A. Glande odorifique isolée.

130. Thte et appareil digestif de Trirhomerana fusca.

a. Tête vue de côté pour montrer 
Fis.

la structure singulière de la Juuche.

$b b$. Glandes salivaires.

c. P'anse.

1. Gésier.

1. Ventricule chylifique.

$\int f$. Vaisscaux hépatiques.

y. Iniestin.

h. Rectum.

i. Bout de l'abdomen.

131. Glande salivaire de Spherocerct subsullans.

13:. Rectum de l'Ochtera mantis pour faire voir la glande singulière qui s'y Irouve.

133. Otul de ce Diptère.

134. Têle et appareil digestif de Phork pullipes.
a. Tête avec antennes, palpes, trompe étalés.
bb. Glandes salivaires.
c. Panse.
d. Ventricule chylifique.
re. Vaisseaux hépatiques terminés par une vésicule.
f. Intestin.
g. Rectum.
h. Bout de l'abdomen.

135. Détails de structure extérieure de Cheligaster putris.
4. Abdomen avec les pinceaux du mâle.
b. Antenne.
c. Soie de l'anteune.
d. Patte antérieure du màle.
e. Crochet du tarse.
f. Patte antéricure de la femelle.

136. Appareil génital mâle de Nemopoda cylindrica.

au. Testicules.
b6. Vésicules sirminales
c. Vésicule séminale impaire.
d. Canal fjaculateur.
c. Riectum.
d. Glande odorifique.
g. Armure copulatrice.

137. Pièce sous-abdominale du màte formant un organe copulateur accessoire.

138. Appareil génital maite de Drosophilu fuscialu.

au. Testicules.

$b b$. Vésicules séminales.

c. Canal éjaculateur.

d. Armure copulatrice.

139. Appareil génital femelle de Platysloma ambrarum.

au. Ovaires fécondés.

66. Réservoirs de la glande sébifique.

c. Rectum.

d. Oviscapte.

A. Gaîne ovigère et cuf.

140. Le mème organe non fécondé el étalé, pour mettre en évidence les connexions de ses parties.
aa. Ovaires.
bb. Orbicelles.
cc. Réservoirs séminanx.
d. Oviducte.
A. Un orbicelle isolé,

141. Appareil génital femelle d'Ulidia demandata.
aq. Ovaires fécondés.
bb. Calices postérieurs.
cc. Orbicelles.
dd. Réservoirs séminaux.
e. Oviducte. 
Fig.

f. Oviscapte.

A. Gaine ovigère isoléc.

142. Glande sébifique isolée et vue pardessous pour montrer ses connexions:

aa. Orbicelles réunis en un seul tronc.

bb. Réservoirs séminaux.

c. Oviducte.

d. Cols des ovaires.

143. Appareil génital femelle de Sepedon sphegeus.

$a a$. Ovaires.

bb. Calices postérieurs.

c. Oviducte.

dd. Glande sébifique.
Fig.

c. Derniers segments de labdomen.

A. Gaine ovigère isolée.

B. Portion de la' glande sébifique isolée.

144. Appareil génital femelle de Cheligaster putris.

aa. Ovaires fécondés.

$b$. Ligaments suspenseurs en faisceaux.

c. Oviducte.

d. Glande sébifique.

c. Réservoir ovigère.

f. Oviscapte.

A. Gaine ovigère isolée.

B. Ovaire vierge.

C. Oviscapte isolé. 

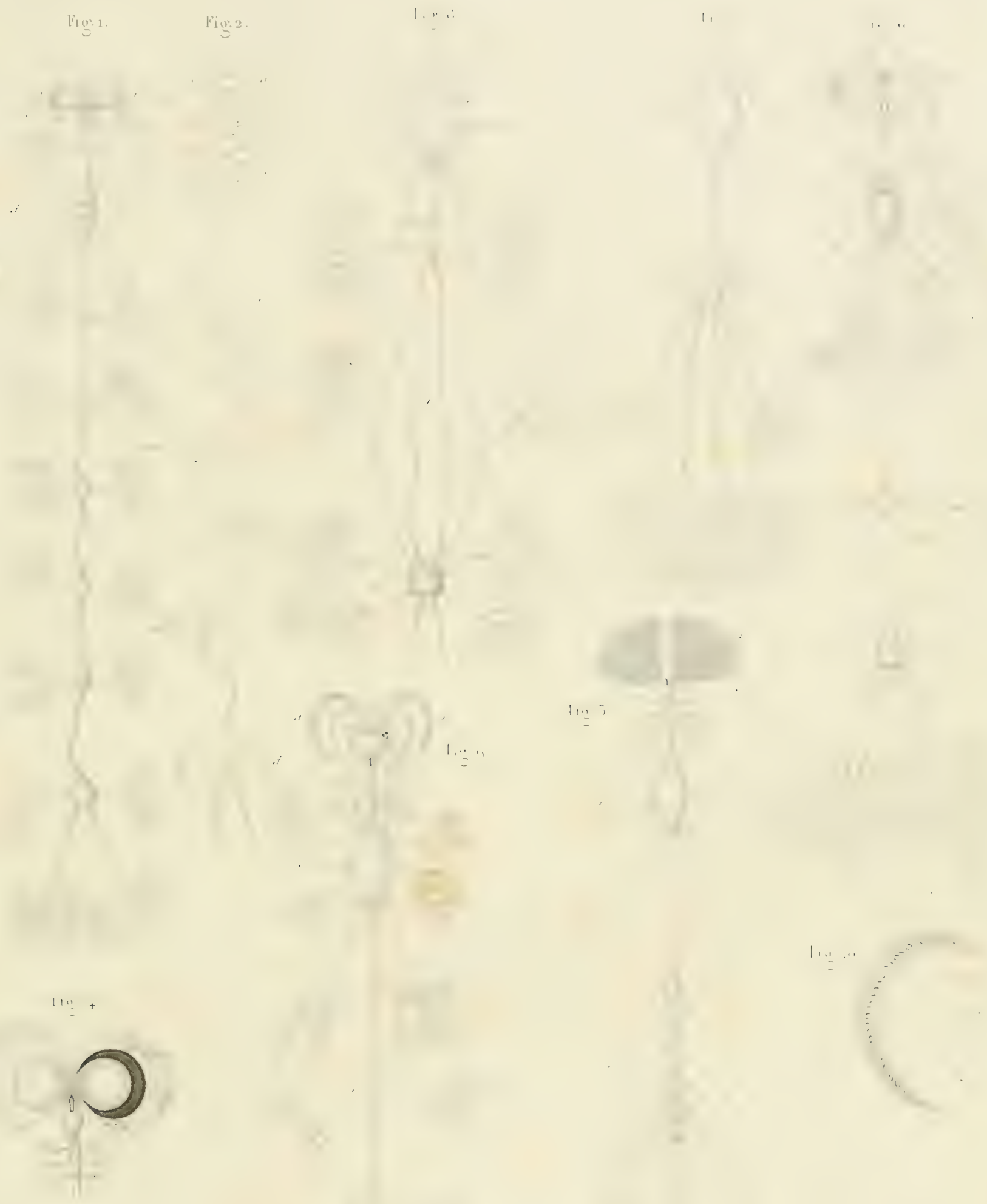

1,113

$11: 11$ 



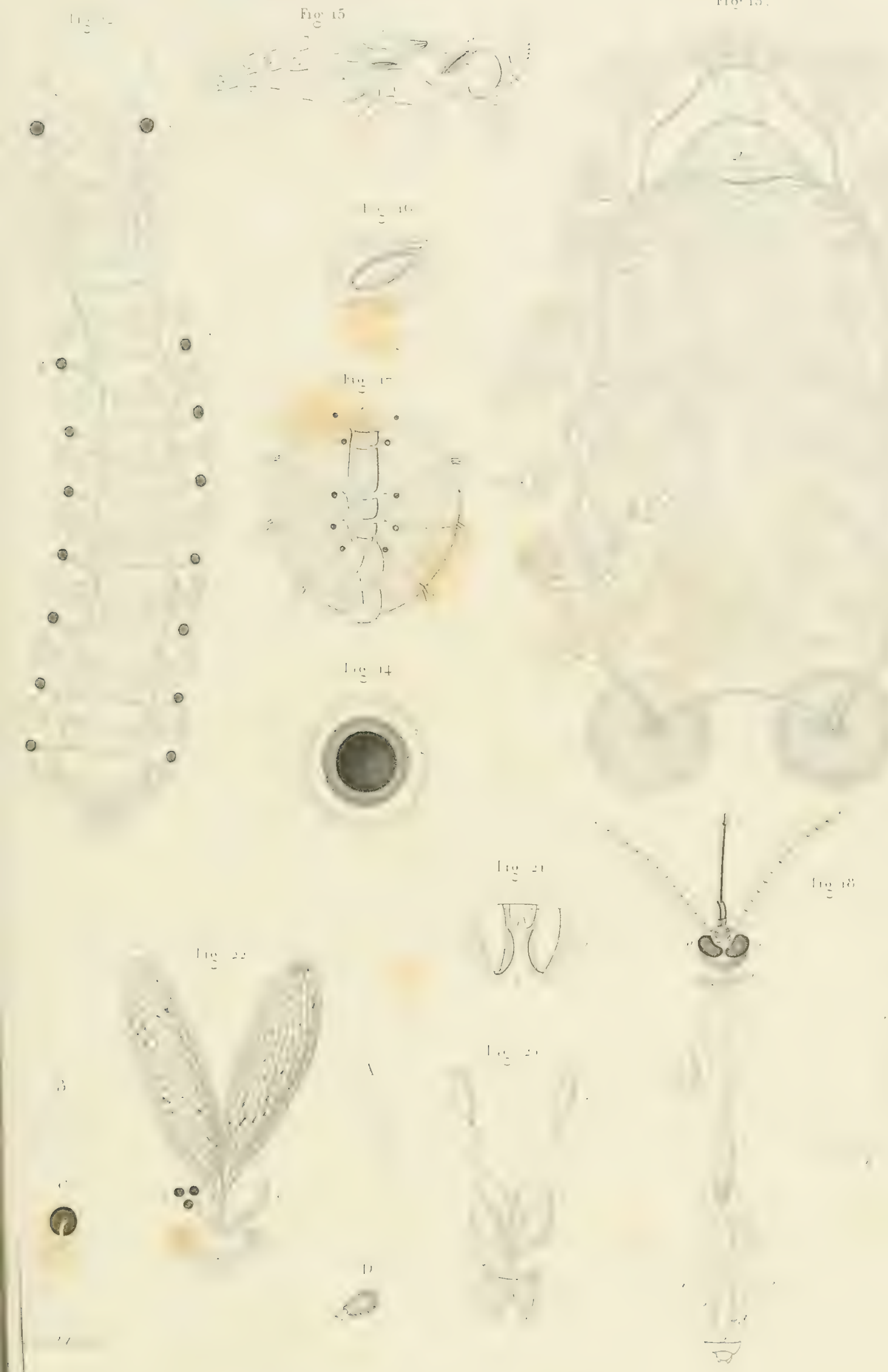


Fid 23
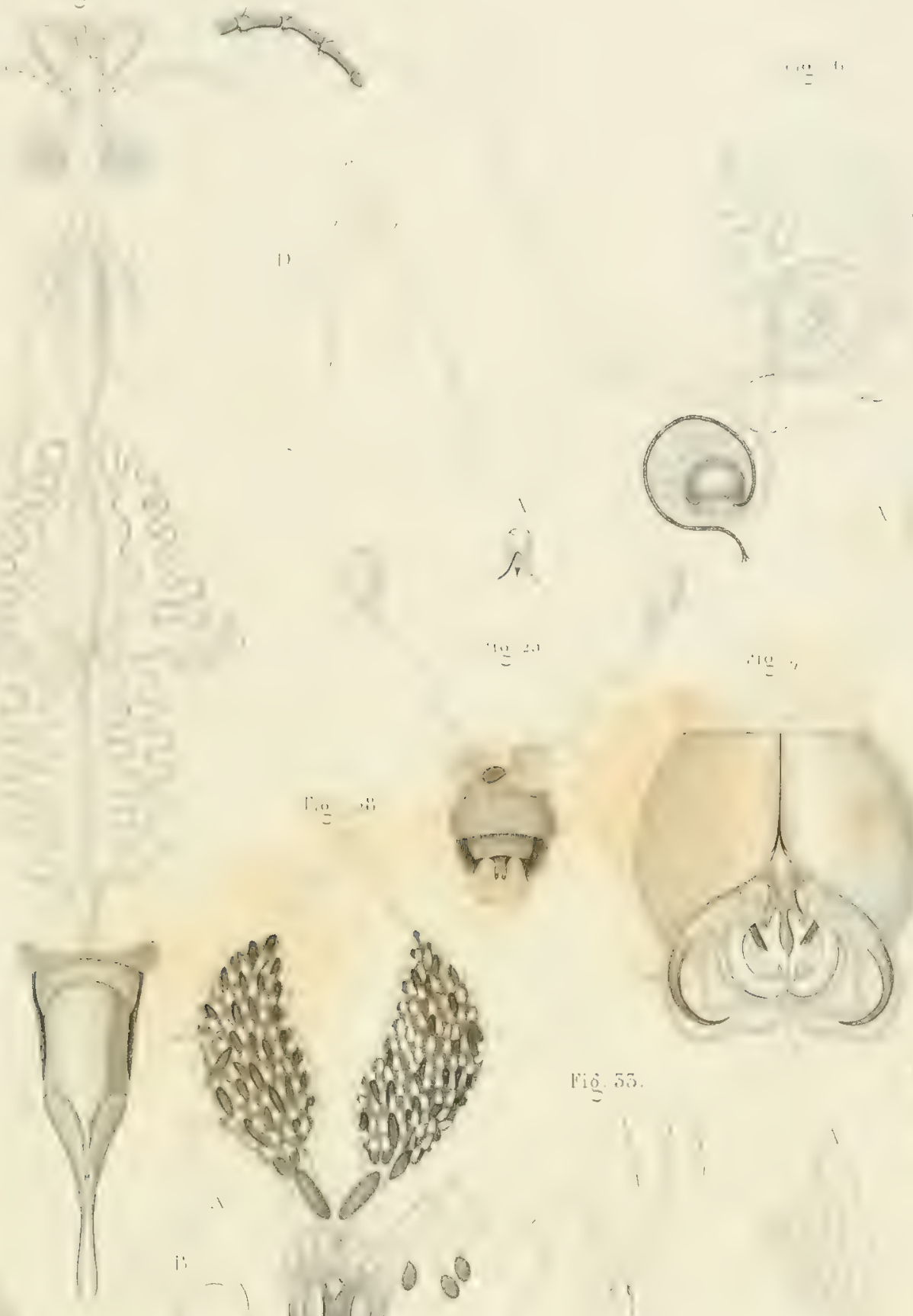

rig $\overline{3} \overline{3}$
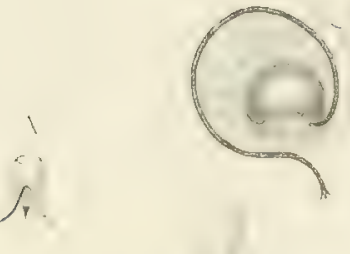


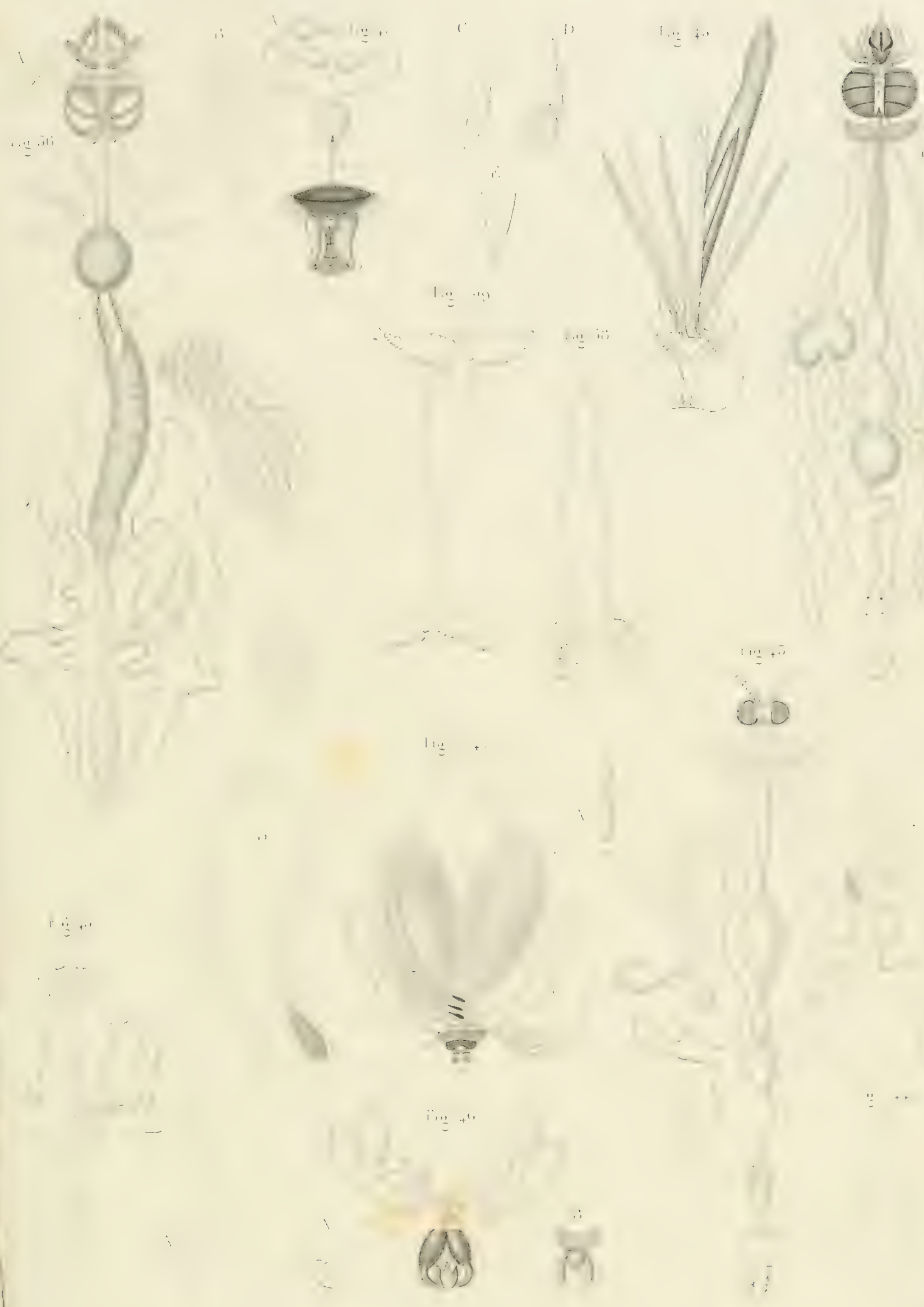


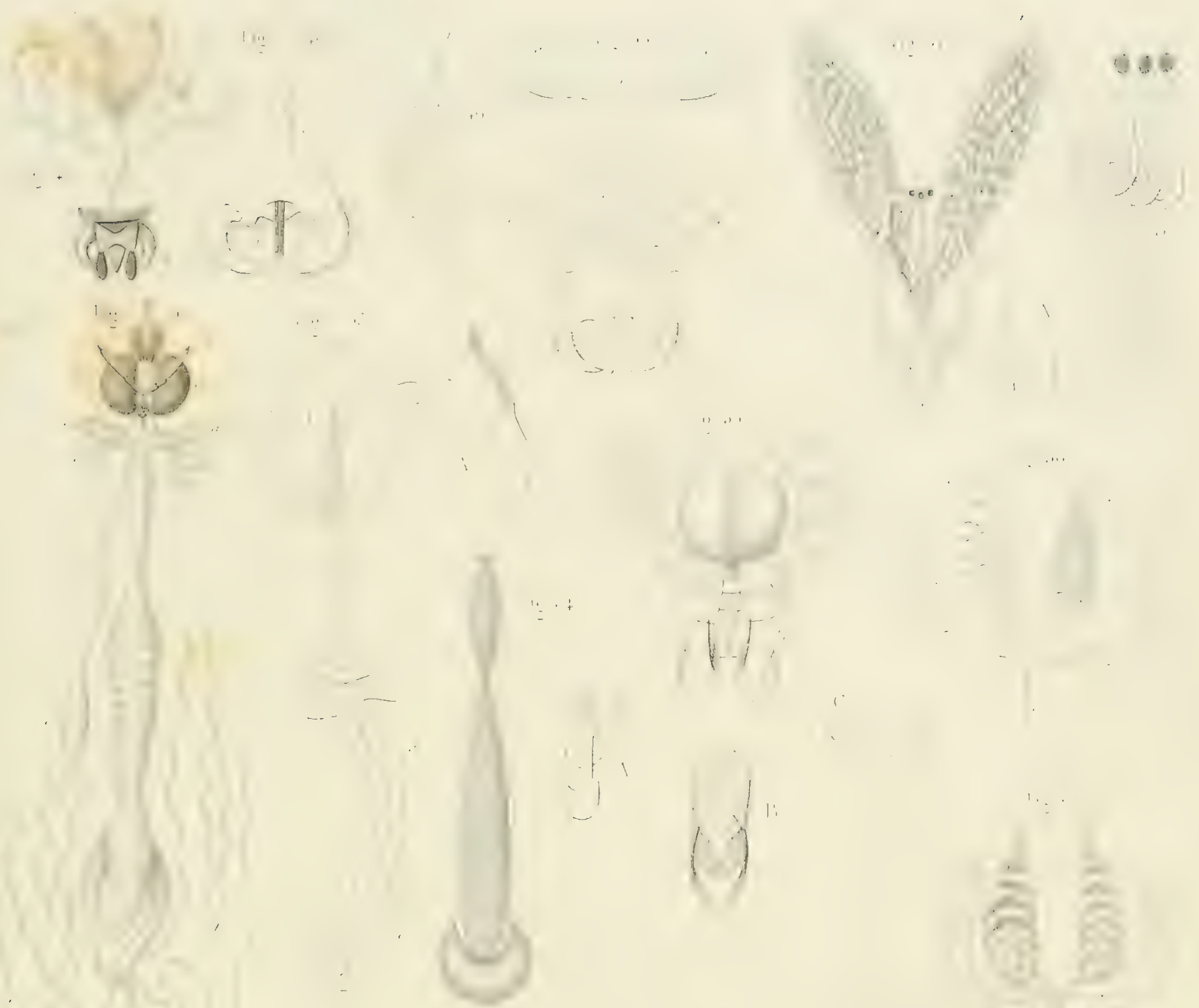

$+1$
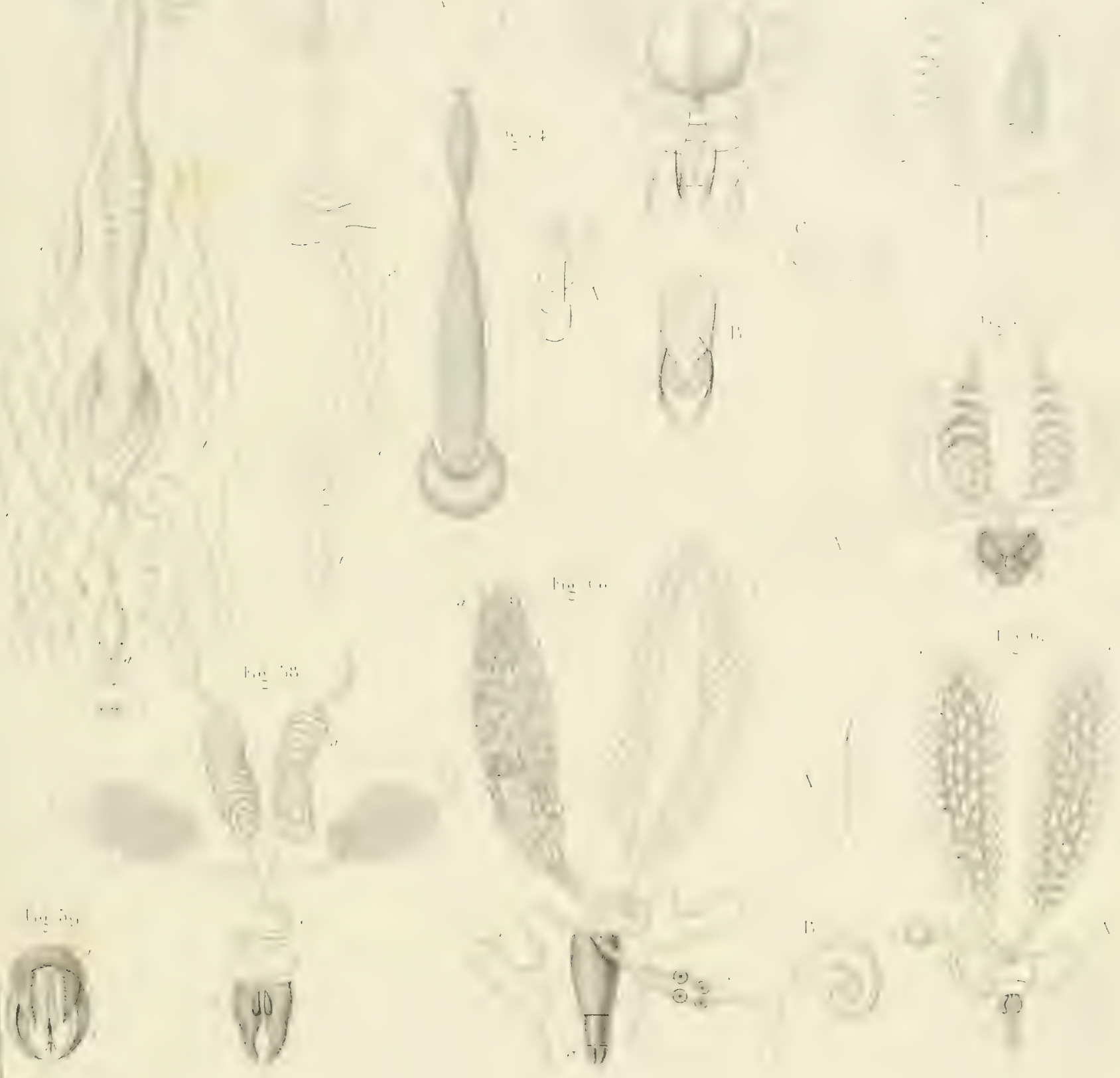


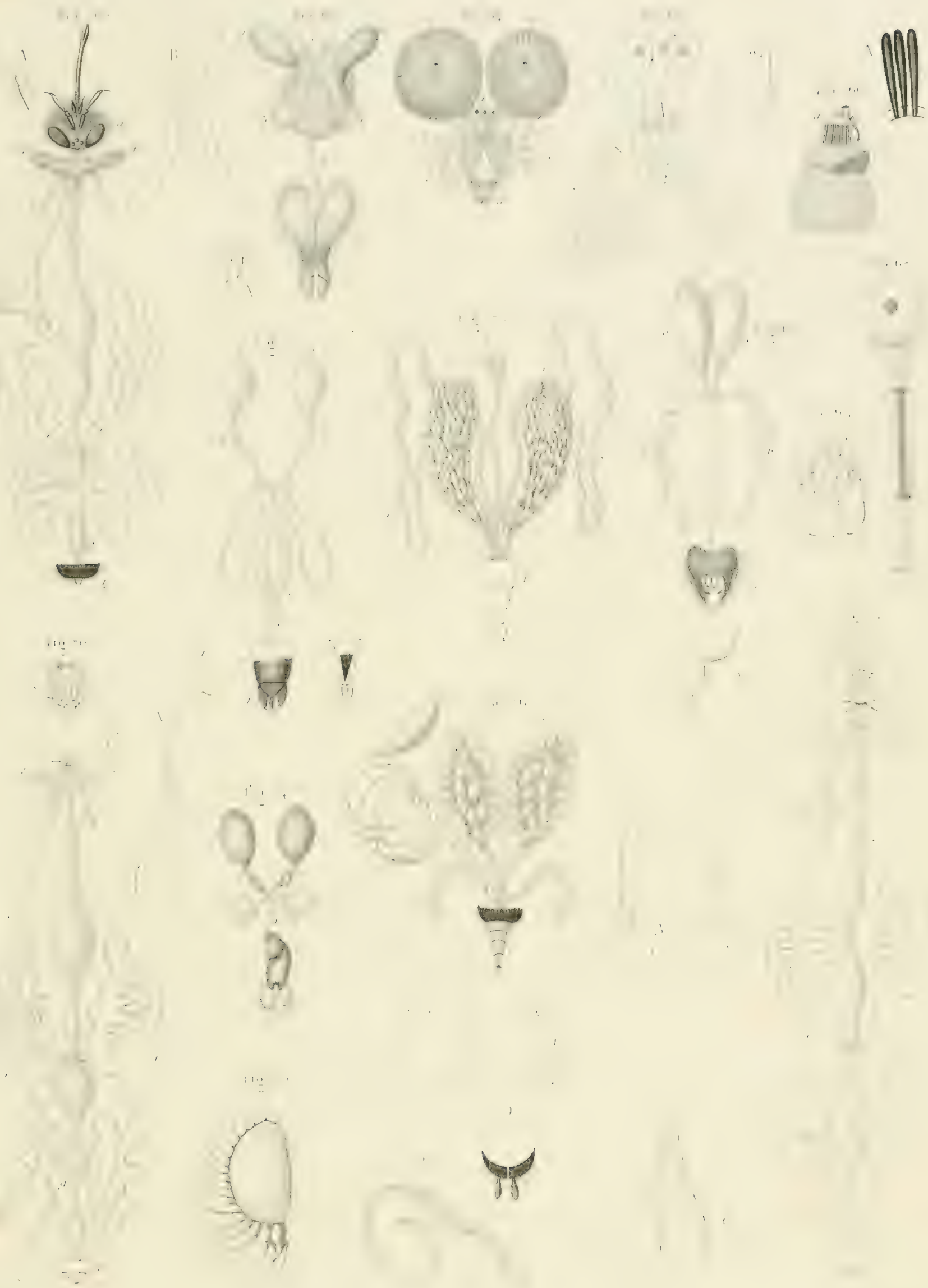



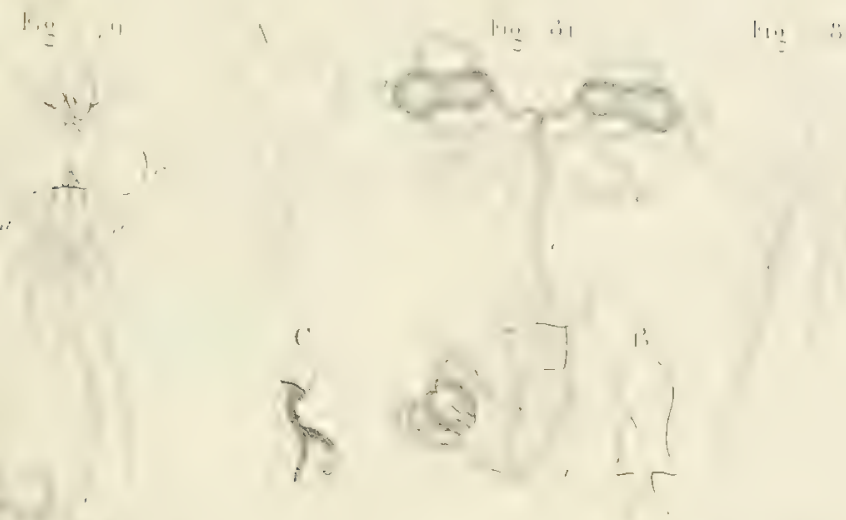

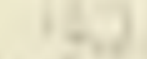
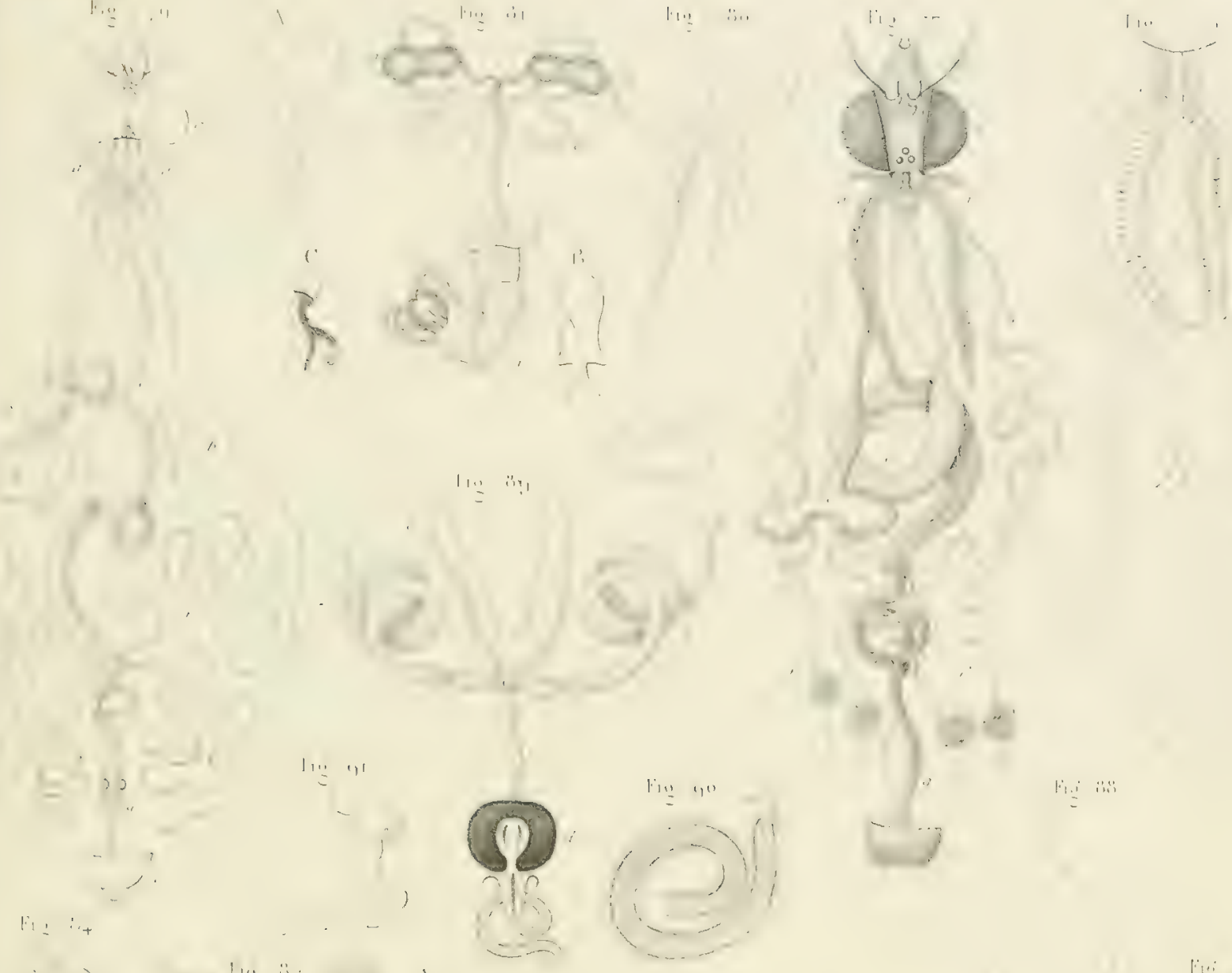

$11,:-3$

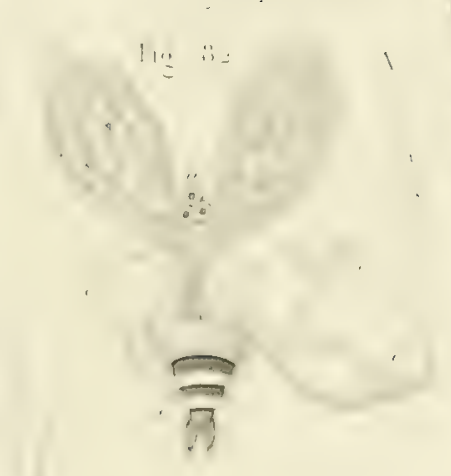

(1) li
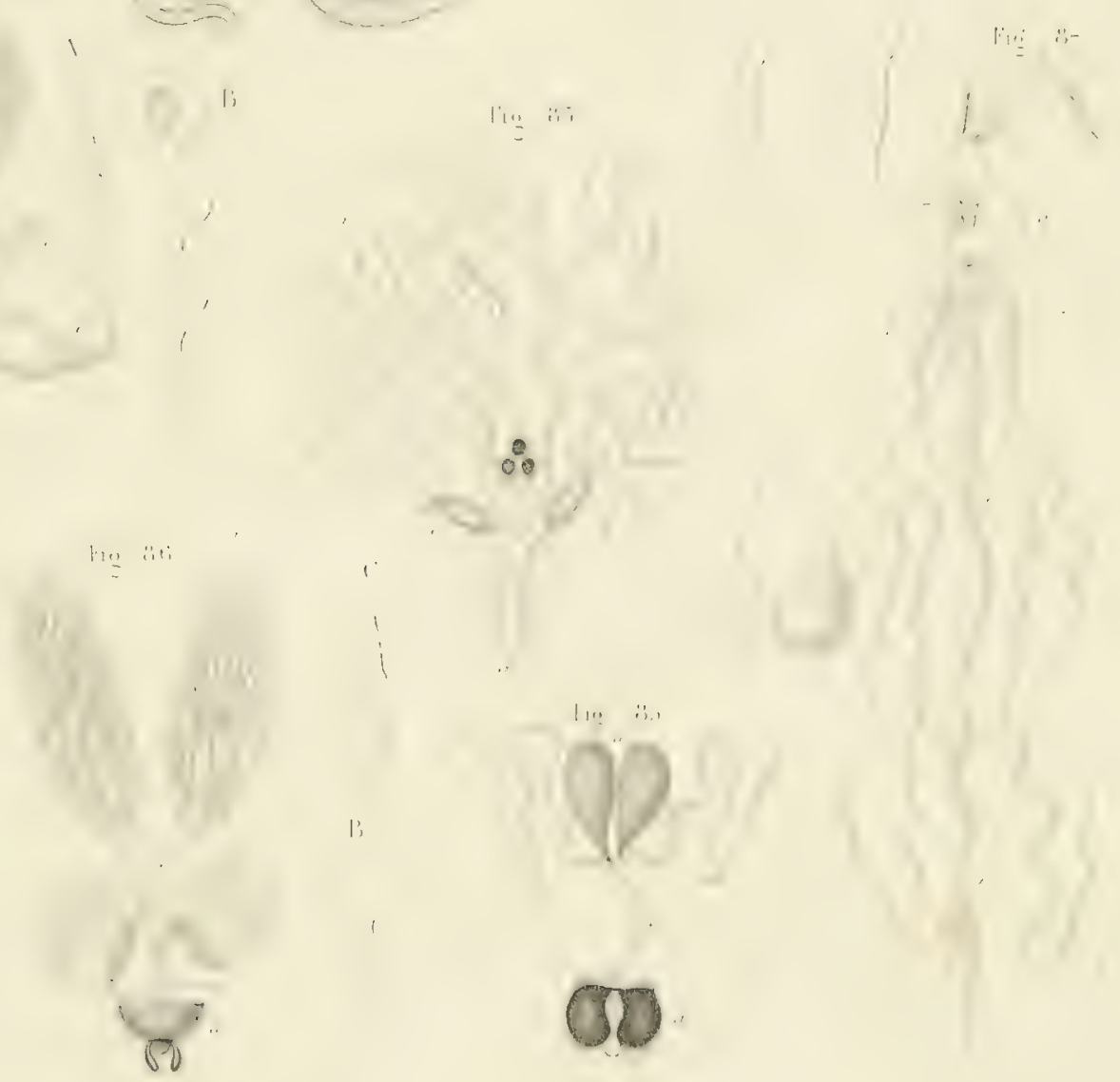

I.

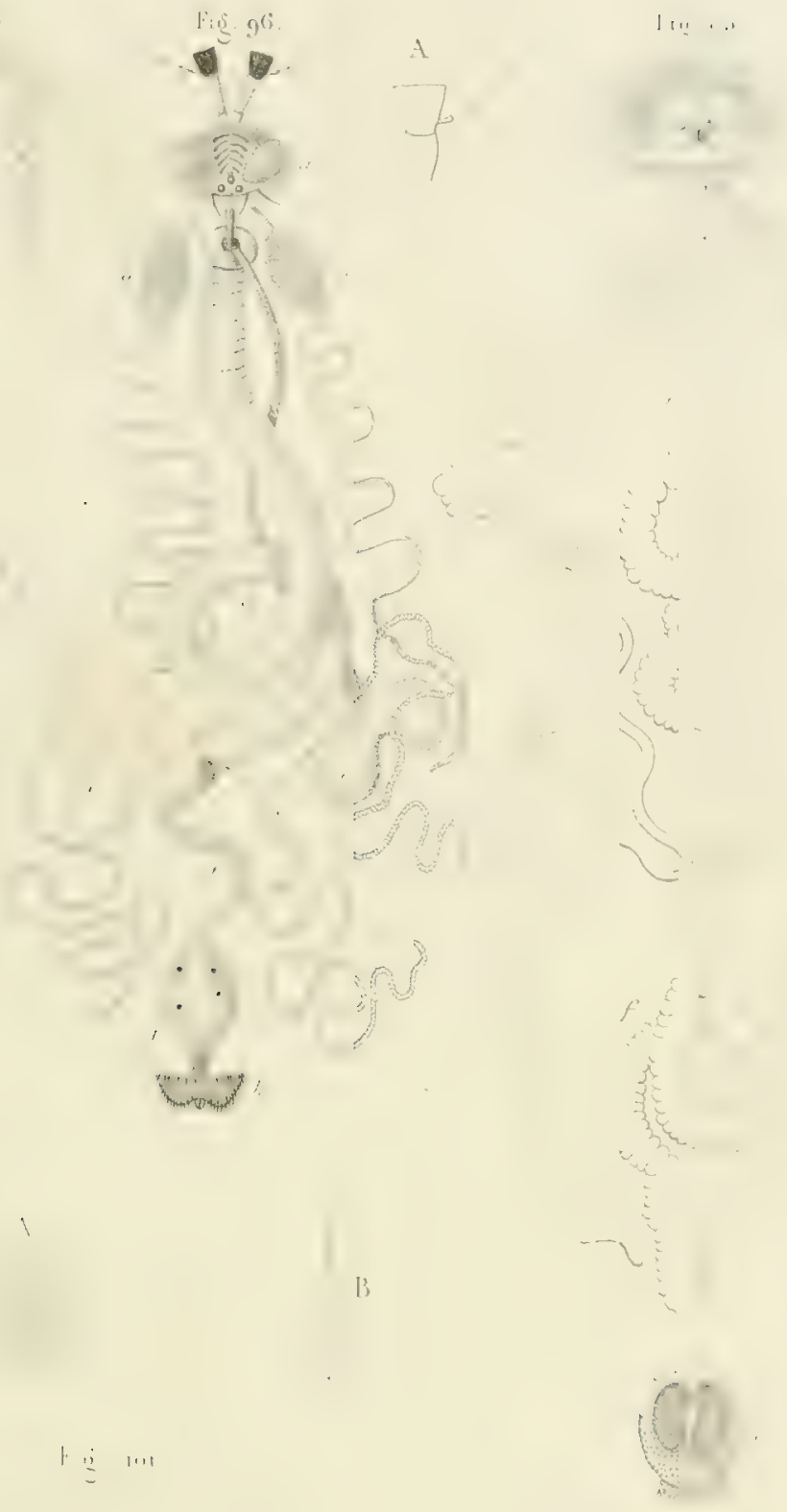

lin $: \cdots$

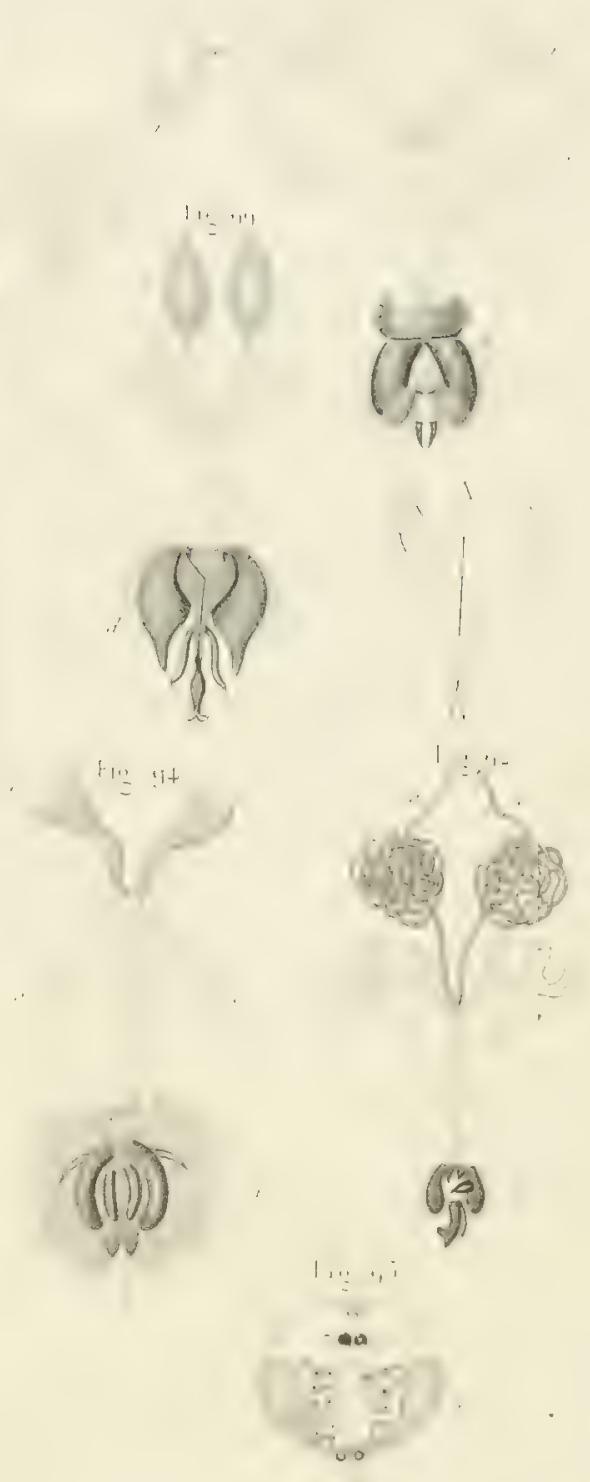

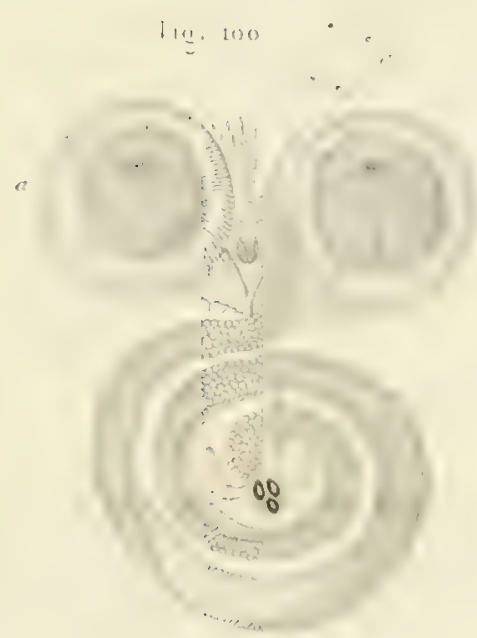




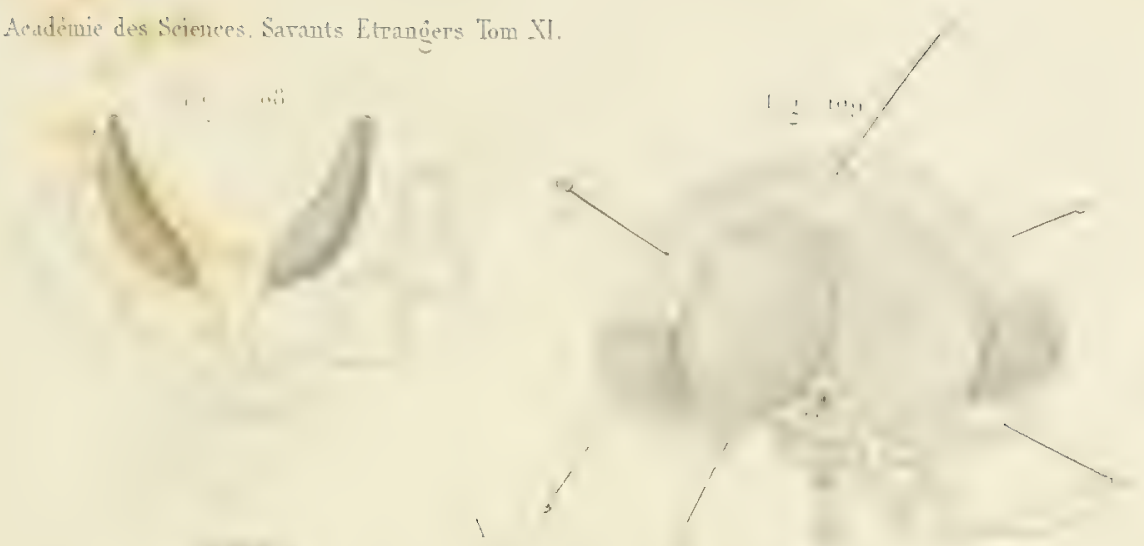

(i) $=0+$

(ii)

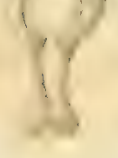

H...

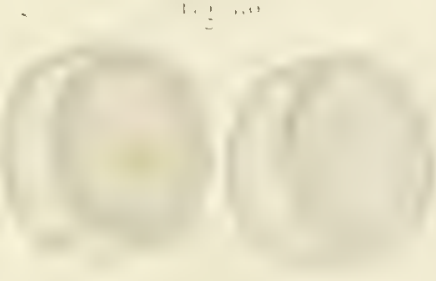

$\sin$

\section{ivi}

8

$\operatorname{lig} 40$
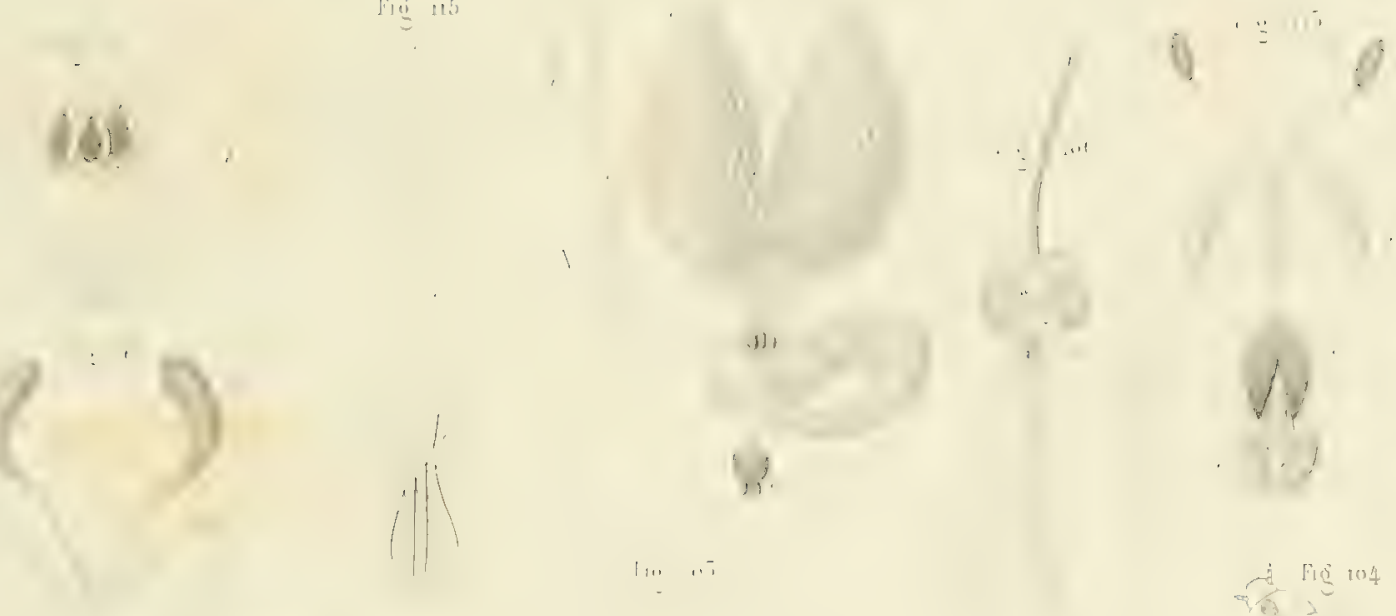

$\because \cdots$
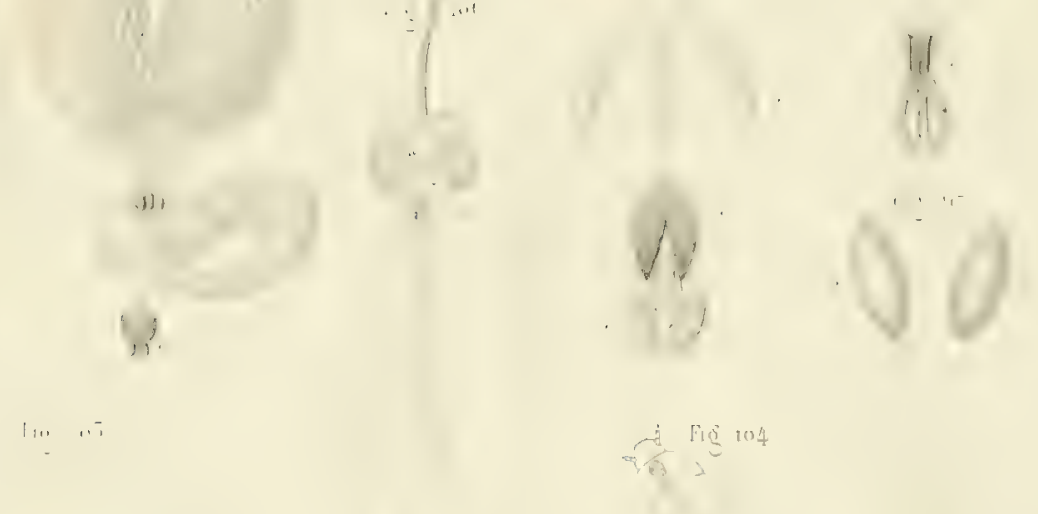

\section{0}
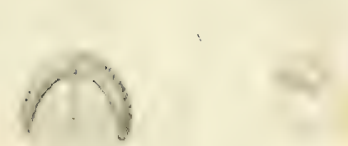

1.4.

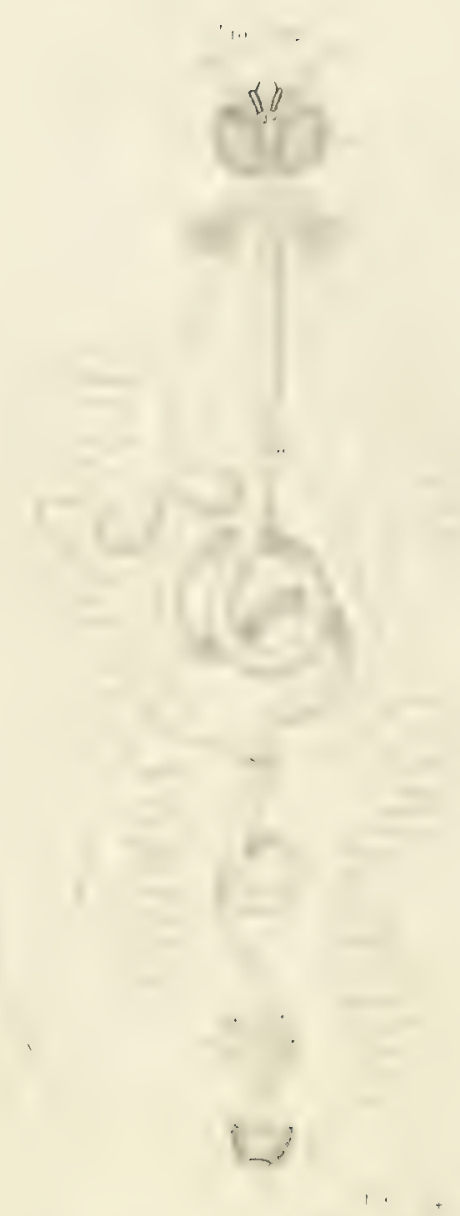

$$
\text { (1) }
$$
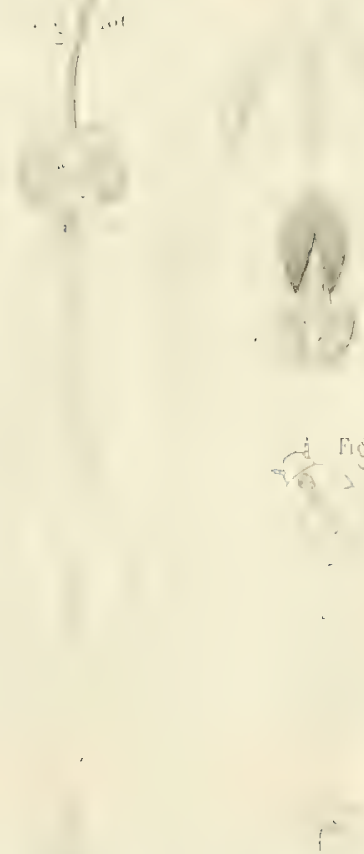


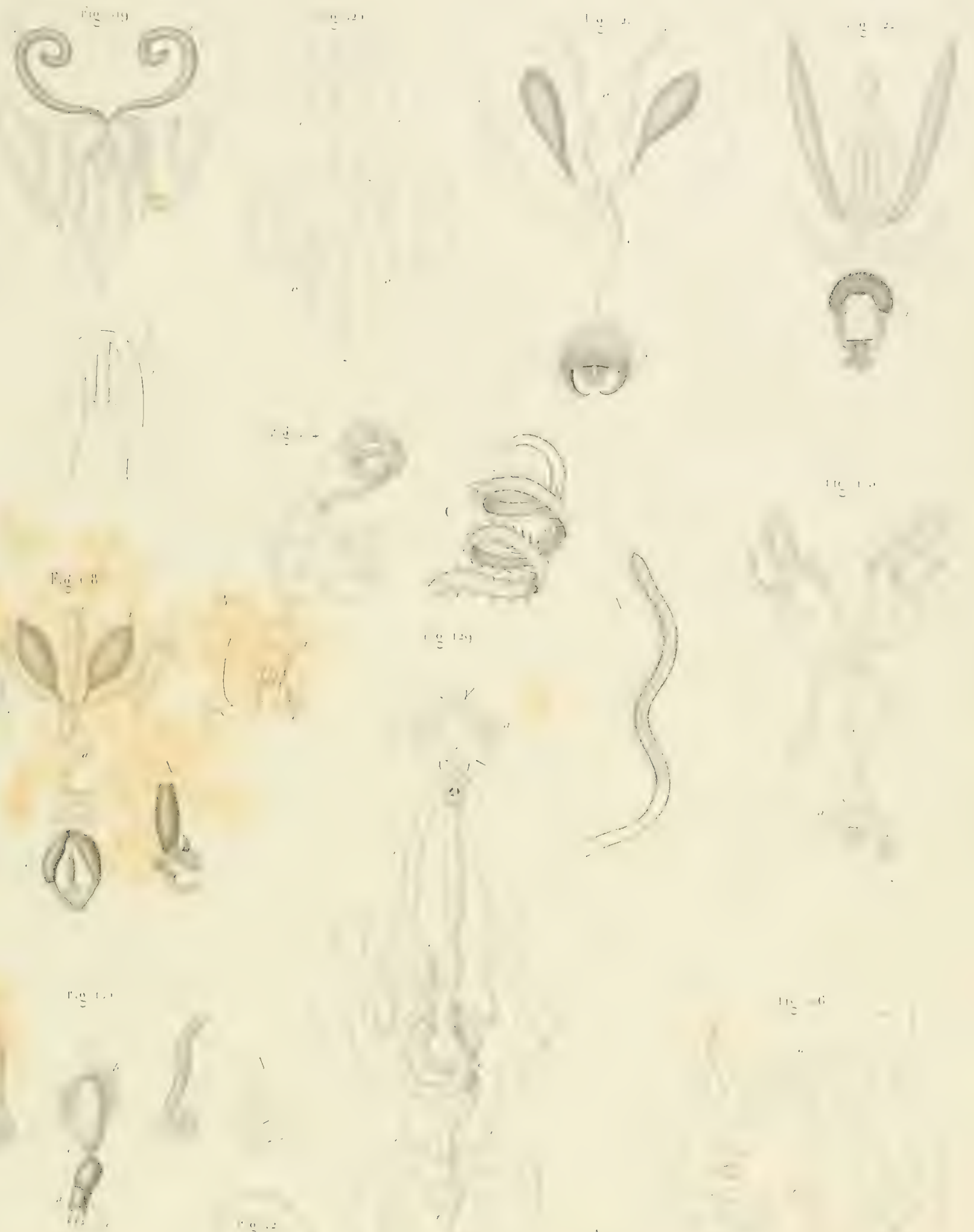


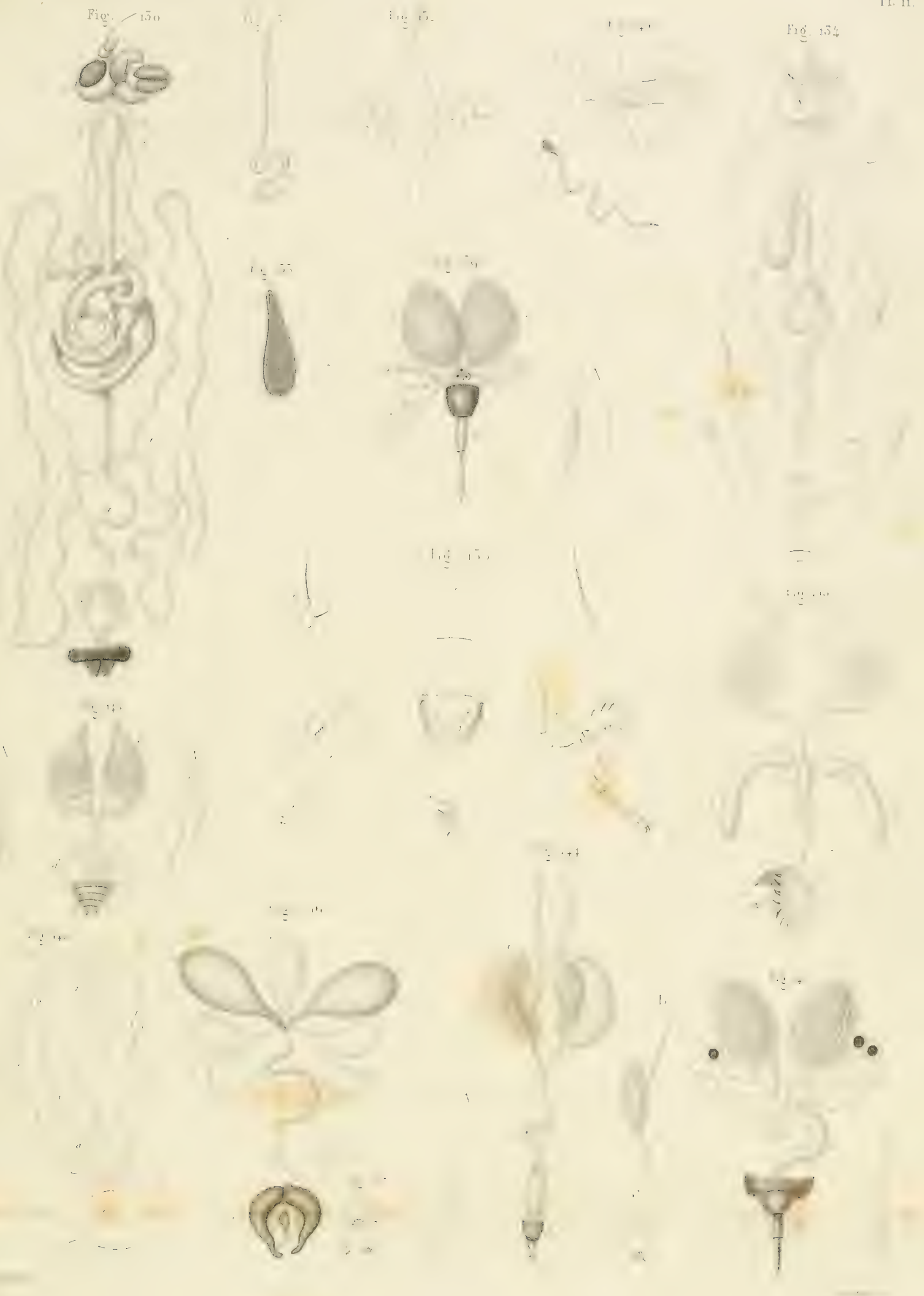

$1, \cdots$ 
- 




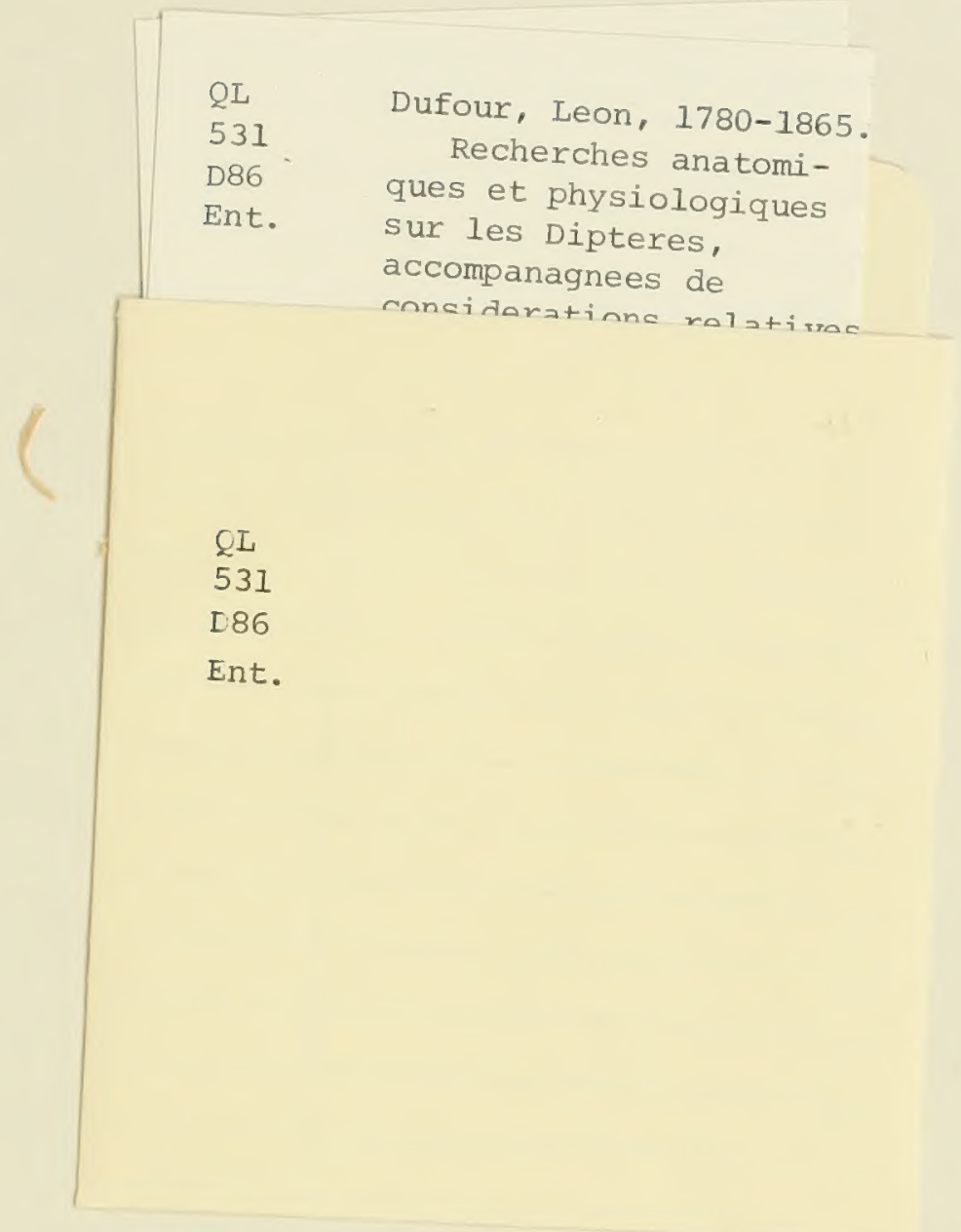


